\title{
Untersuchungen zur Synthese von Yohimban- und Campthoteca-Alkaloiden durch Domino-Knoevenagel-Hetero-Diels-Alder-Reaktion
}

\author{
Dissertation \\ zur Erlangung des Doktorgrades \\ der Mathematisch-Naturwissenschaftlichen Fakultäten \\ der Georg-August-Universität zu Göttingen
}

\author{
vorgelegt von \\ Katharina Anna Klapa \\ aus Kowary/Polen
}

Göttingen 2007 
D 7

Referent:

Prof. Dr. Dr. h.c. L. F. Tietze

Korreferent:

Prof. Dr. H. Laatsch

Tag der mündlichen Prüfung: $\quad$ 17.01.2007 
Die vorliegende Arbeit wurde in der Zeit von November 2003 bis Februar 2007 unter der Leitung von Prof. Dr. Dr. h.c. L. F. Tietze am Institut für Organische und Biomolekulare Chemie der Universität Göttingen angefertigt.

Mein besonderer Dank gilt Prof. Dr. Dr. h. c. L. F. Tietze für die interessante Themenstellung sowie für die Unterstützung und Förderung während des Fortgangs dieser Arbeit. 
Meiner Familie 


\section{INHALTSVERZEICHNIS}

\section{ALLGEMEINER TEIL}

INHALTSVERZEICHNIS I

ALLGEMEINER TEIL I

1. Einleitung 1

2. Alkaloide 3

2.1. Allgemeines 3

2.2. Klassifizierung der Alkaloide 5

2.3. Heterocyclische Alkaloide 6

2.3.1. Indol-Alkaloide 6

2.3.2. Yohimban-Alkaloide 7

2.3.2.1. Biosynthese von Yohimbin 8

2.3.2.2. Totalsynthese von Yohimbin (9) 11

2.3.3. Chinolin-Alkaloide 13

2.3.4. Camptotheca-Basen 14

2.3.4.1. Biosynthese von Camptothecin (44) 15

2.3.4.2. Totalsynthese von Camptothecin (44) 15

3. Theorie der Diels-Alder-Reaktion 19

3.1 Allgemeines 19 
3.2. Mechanismus _ 25

3.3. Die Klopman-Salem-Gleichung ___ 27

3.4. Einteilung der Diels-Alder-Reaktionen __ 30

3.5. Regioselektivität __ 33

3.6. Stereoselektivität__ 36

4. Domino-Reaktionen _ 38

4.1. Das Konzept der Domino-Reaktion __ 38

4.2. Domino-Reaktionen in der organischen Synthese ___ 40

4.2.1. Domino-Knoevenagel-Hetero-Diels-Alder-Reaktion ___ 41

5. Problemstellung und Planung der Arbeit___ 44

$5.1 \quad$ Einleitung _ 44

5.2. Syntheseplanung __ 46

A) Versuche zur Synthese von Yohimin (9)__ 52

6. Synthese des Enolethers 122

6.1. Versuche zur Synthese des Enolethers 122

6.2. Synthese des Enolethers $122 \_54$

6.3. Versuche zur Synthese weiterer Enolether __ 58

7. Synthese des Aldehyds 121

7.1. Versuche zur Synthese des enantiomerenreinen (1S)-Tetrahydroisochinolins 121

7.2. Diskussion der spektroskopischen Daten der Verbindung 174 72

8. Die Domino-Knoevenagel-hetero-Diels-Alder-Reaktion __ 74

8.1. Disskussion des Reaktionsmechanismus __ 74

8.2 Die biomimetische Cyclisierung___ 76 
8.3. Untersuchungen zur Domino-Knoevenagel-hetero-Diels-Alder-

Reaktion 78

B) Versuche zur Synthese von Camptothecin 82

9. Versuche zur Synthese des enantiomerenreinen Aldehyds 130 82

10. Versuche zur Synthese des Silylenolethers 129 86

11. Zusammenfassung 89

\section{EXPERIMENTELLER TEIL}

1. Allgemeine Arbeitstechniken 101

2. Allgemeine Arbeitsvorschriften (AAV) 105

A) Versuche zur Synthese von Yohimban (9) _ 108

3. Synthese des chiralen Hydrierungskatalysatorliganden _ 108

3.1. (1S,2S)-N-Tosyl-1,2-diphenylethylendiamin (224) __ 108

3.2. (1R,2R)-N-Tosyl-1,2-diphenylethylendiamin (224)___ 109

3.3. Ru-Dimer für die Synthese von Katalysator 125 109

4. Synthese des Aldehyds 121 110

4.1. Carbethoxysäure (126) 110

4.2. 1-Carbethoxymethyl-1,2,3,4-tetrahydro- $\beta$-carbolin (123) 111

4.3. 1-Carbethoxymethyl-2-benzyloxycarbonyl-1,2,3,4-tetrahydro- $\beta$ carbolin (173)

4.4. Benzyl-1-(2-oxoethyl)-3,4-dihydro-1H-pyrido[3,4-b]indol-2(9H)- 
carboxylat (121)

5. Synthese des Aldehyds 118

5.1. 2-Benzyl-9-tert-butyl-1-(2-ethoxy-2-oxoethyl)-3,4-dihydro-1Hpyrido[3,4-b]indol-2,9-dicarboxylat (174)

5.2. 2-Benzyl-9-tert-butyl-1-(2-oxoethyl)-3,4-dihydro-1H-pyrido[3,4-b] indol-2,9-dicarboxylat (118) 116

6. Synthese des enantiomerenreinen Esters S-123 118

6.1. Ethyl-2-(4,9-dihydro-3H-pyrido[3,4-b]indol-1-yl)acetate (124) 118

6.2. (S)-Ethyl-2-(2,3,4,9-tetrahydro-1H-pyrido[3,4-b]indol-1-yl) acetate (123) 118

7. Synthese der Imine 167 und 168 119

7.1. 2-(2,3,4,9-Tetrahydro-1H-pyrido[3,4-b]indol-1-yl)ethanol (164) 119

7.2. 1-(2-(Triisopropylsilyloxy)ethyl)-2,3,4,9-tetrahydro-1H-pyrido[3,4-b] indol (166)

7.3. 1-(2-(tert-Butyldimethylsilyloxy)ethyl)-2,3,4,9-tetrahydro-1H-pyrido $[3,4-b]$ indol (165)

7.4. 1-(2-(Triisopropylsilyloxy)ethyl)-4,9-dihydro-3H-pyrido[3,4-b] indol (167)

7.5. 1-(2-(tert-Butyldimethylsilyloxy)ethyl)-4,9-dihydro-3H-pyrido[3,4-b] indol (168)

8. Synthese des Modelsystems 172 zur Überprüfung der Noyori-

Reduktion 124

8.1. N-(3,4-Dimethoxyphenyl)ethylacetamid (170) 124

8.2. 6,7-Dimethoxy-1-methyl-3,4-dihydroisochinolin (171) 125 
8.3. (S)-6,7-Dimethoxy-1-methyl-1,2,3,4-tetrahydroisochinolin (172) 126

9. Synthese der Acetale 127

9.1. 5-(tert-Butyldimethylsilyloxy)pentan-1-ol (132) 127

9.2. 5-( tert-Butyldimethylsilyloxy)pentanal (133) 128

9.3. tert-Butyl-(5,5-dimethoxypentyloxy)dimethylsilan (134) 129

9.4. (5-(Benzyloxy)pentyloxy)-(tert-butyl)dimethylsilan (137) 130

9.5. 5-(Benzyloxy)pentan-1-ol (136) 131

9.6. 5-(Benzyloxy)pentanal (138) 132

9.7. ((5,5-Dimethoxypentyloxy)methyl)benzol (139) 133

9.8. 4,5-Dihydroxypentylacetat (163) 134

10. Synthese des Dienophils 122 135

10.1. Tetrahydrofuran-2-ol (141) 135

10.2. 5-Methoxypent-4-en-1-ol (148) 135

10.3. 1-Benzyloxy-5-methoxypent-4-en (122) 136

B) Versuche zur Synthese von Camptothecin 44 138

11. Synthese des Modelsystems 218 zur Überprüfung der Totalsynthese von Camptotecin 138

11.1. 2-(3,4-Dihydroisochinolin-1(2H)-yliden)essigsäureethylester (214) 138

11.2. 2-(1,2,3,4-Tetrahydroisochinolin-1-yl)essigsäureethylester (215) 139

11.3. 2-(1,2,3,4-Tetrahydroisochinolin-1-yl)ethanol (216) 140

11.4. Benzyl-1-(2-hydroxyethyl)-3,4-dihydroisochinolin-2(1H)carboxylat (217)

11.5. Benzyl-1-(2-oxethyl)-3,4-dihydroisochinolin-2(1H)-carboxylat (218) _ 142

12. Versuche zur Synthese des Dienophils 129 
12.1. 2-Oxobuttersäuremethylester (219) 144

12.2. 2-Acetoxybuttersäure (223) 144

13. Versuche zur Synthese des Aldehyds 130 146

13.1. 4-Oxo-4-(phenylamino)but-2-ensäureethylester (200) 146

13.2. 4-Oxo-4-(phenylamino)-3-(prop-2-ynylamino)buttersäureethyl ester (202) 147

13.3. 3-(Benzylosycarbonyl)(prop-2-ynyl)amino)-4-oxo-4-

(Phenylamino)buttersäureethylester (203) 148

13.4. 3-Aminoprapansäureessigester (208) 149

\section{ANHANG}

1. Verwendete Abkürzungen und Akronyme 150

2. Literaturverzeichnis 153

3. Danksagung 164

4. Lebenslauf 165 


\section{ALLGEMEINER TEIL}

\section{Einleitung}

Seit Wöhler im Jahre 1828 die gezielte Darstellung von Harnstoff gelungen ist, ${ }^{[1]}$ hat sich die oganische Chemie in einem solchen Maße weiterentwickelt, daß man annehmen mag, der Mensch könne jede beliebige Substanz herstellen. ${ }^{[2]}$ Die größten Herausforderungen an deren Synthese gehen dabei vom Gegengenstand der medizinischen Forschung aus, von Krankheiten wie Krebs, AIDS, Diabetes und SARS. Oft ist es die Natur die uns den Weg weist und so begründet sich das rege Interesse an der Isolierung unbekannter Naturstoffe auf der Annahme, daß jedes natürliche Molekül auch eine Funktion besitzt und daß sich gegen viele Krankheiten auf dieser Welt in der Natur selbst ein geeignetes Gegenmittel finden lassen sollte. Allerdings ist die Isolierung größerer Mengen von Substanzen aus natürlichen Qellen oft problematisch. So war z. B. die immergrüne pazifische Eibe Taxus brevifolia durch den Bedarf an Taxol (0.004\% Taxol in der Rinde), einem potenten Antitumorwirkstoff gegen Leukämie und Brustkrebs, zeitweilig vor dem Aussterben bedroht. ${ }^{[3]}$ Die Behandlung nur eines Patienten erfordert sechs 100 Jahre alte Bäume. Aber auch die Synthese pharmakologisch interessanter Stoffe wie Taxol, ${ }^{[4]}$ Palytoxin, ${ }^{[5]}$ Brevetoxin, ${ }^{[6]}$ Erythromycin ${ }^{[7]}$ und Vancomycin ${ }^{[8]}$ liefert den Wirkstoff zunächst nur in analytischen Mengen. Die Totalsynthese von Taxol nach R. A. Holton ${ }^{[9]}$ mit 59 Schritten ergibt das Chemotherapeutikum in einer Gesamtausbeute von ca. $1 \%$. Heute, nach umfassender Forschung, wird Taxol in einem ebenfalls von R. A. Holton entwickelten semisynthetischen Verfahren aus 10-Deacetylbaccatin hergestellt, dass das Taxol-Grundgerüst besitzt und in großen Mengen ohne Abholzung aus Blättern der englischen Eibe gewonnen werden kann. Solche Möglichkeiten bieten sich aber nicht immer. Daher ist die Entwicklung neuer, effizienter und selektiver Synthesemethoden von Bedeutung. ${ }^{[10]}$ Großes Interesse kommt dabei den Domino-Reaktionen ${ }^{[11]} \mathrm{zu}$, die unter gleichzeitiger Beachtung 
atomökonomischer Aspekte ${ }^{[12]}$ der Forderung Rechnung tragen, die anfallende Menge an Abfall und Lösungsmitteln zu minimieren, die Umwelt zu entlasten und Kosten zu sparen. Weiterer Forschungsschwerpunkt liegt auf der Enwicklung chiraler Katalysatoren, ${ }^{[13]}$ deren Effizienz und Selektivität an enzymatische Systeme heranreicht oder diese sogar übertrifft. Die chirale Information eines Moleküls besitzt einen entscheidenen Einfluß auf die physiologische Wirkung wie sich beispielsweise bei der Verabreichung des racemischen Thalidomid (Contergan) ${ }^{[14]}$ zeigte. Während das eine Enantiomer die erwünschte sedierende Eigenschaft besitzt, führte die teratogene Wirkung des anderen Enantiomers zu den verheerenden fötalen Mißbildungen bei schwangeren Frauen. ${ }^{[15]}$ Aus diesem Grunde ist die Synthese enantiomerenreiner Verbindungen von großer zentraler Bedeutung. Die unterschiedliche Wirkungsweise von Stereoisomeren stellt wiederum neue Herausforderungen an die organische Synthese dar, da sie zur Herstellung von Stereoisomeren eines Naturstoffes sowie dessen Analoga und Derivate die aus dem Naturstoff selbst nicht zugänglich sind herangezogen wird. ${ }^{[16]} \mathrm{In}$ der vorliegenden Arbeit wird untersucht, wie aufbauend auf der von L. F. Tietze entwickelten Domino-Knoevenagel-Hetero-Diels-Alder-Reaktion ein neuer Zugang zu Yohimban- und Camptotheca-Alkaloiden geschaffen werden kann. ${ }^{[17]}$ 


\section{Alkaloide}

\subsection{Allgemeines}

Der Begriff „Alkaloid“ leitet sich von den Wörtern Alkali und dem griechischen Ausdruck für Art bzw. Ähnlichkeit ab. Damit wird grundsätzlich eine Substanzklasse beschrieben, die alkaliähnliche Eigenschaften aufweist. Die eigentliche Begriffsbildung „Alkaloid“ wurde erstmals im Jahre 1819 von W. Meissner ${ }^{[18]}$ vorgeschlagen:

„Überhaupt scheint es mir auch angemessen, die bis jetzt bekannten Pflanzenstoffe nicht mit dem Namen Alkalien, sondern Alkaloide zu belegen, da sie doch in manchen Eigenschaften von den Alkalien sehr abweichen, sie würden daher in dem Abschnitt der Pflanzenchemie vor den Pflanzensäuren ihre Stelle finden. “

Einfach ausgedrückt, werden hier Alkaloide als alkalisch reagierende Pflanzenstoffe umschrieben. Zu dieser Zeit wurde dieser Begriff jedoch sehr großzügig ausgelegt. So waren die Möglichkeiten der Strukturaufklärung unbekannter Verbindungen im Wesentlichen auf Derivatisierung, kontrollierten chemischen Abbau und Verbrennungsanalyse beschränkt. Dieser Umstand führte dazu, dass man alle unbekannten Verbindungen, die sich neben basischen Eigenschaften auch durch physiologische Aktivität jeglicher Art und Weise auswiesen, unter der Bezeichnung „Alkaloide“ zusammenfasste. Mit zunehmender Zahl der isolierten Alkaloide wurde auch deren Definition erweitert, wie zum Beispiel jene von Hegenauer, ${ }^{[19]}$ die auch $N$-heterocyclische, sich von Aminosäuren ableitende, sauer oder neutral reagierende Verbindungen wie Coffein, Theophyllin und Theobromin mit eingeschloß. ${ }^{[20]}$ Jedoch schien auch diese Erklärung nicht befriedigend, da es sich gezeigt hat, dass sich stickstoffhaltige Heterocyclen und Amine biosynthetisch auf Aminosäuren zurückführen lassen. Erfahrungsgemäß lassen sich alle Alkaloide auf dem Shikimisäure-, dem Polyketid- oder dem Mevalonsäurepfad in Kombination mit einer zumeist aromatischen Aminosäure, zu der Tryptophan, Phenylalanin als auch Tyrosin zählen, aufbauen. Ein Umstand, der folglich auch die Grundstruktur des Ringsystems 
festlegt. ${ }^{[21]}$ Des Weiteren ist heute auch bekannt, dass das Vorkommen der Alkaloide nicht nur auf das Pflanzenreich beschränkt ist. Aufgrund dieser Tatsachen ergibt sich so als geltende Definition: Alkaloide sind stickstoffhaltige organische Verbindungen natürlichen Ursprungs mit mehr oder weniger ausgeprägten basischem Charakter.

Eine Vielzahl an Verbindungen dieser Substanzklasse zeichnet sich durch eine ausgeprägte biologische Aktivität aus, ${ }^{[22]}$ die die Menschheit schon früh zu nutzen wusste (Abbildung 1) So wurde in älteren Kulturen aus Papaver somniferum, dem Schlafmohn, Opium ${ }^{[23]}$ gewonnen, welches bis zur Mitte des 17. Jahrhunderts fast ausschließlich als Medikament zur Schmerzlinderung oder als Schlafmittel eingesetzt wurde. Coniin, das Hauptalkaloid des gefleckten Schierlings, wurde in der Antike zur Vollstreckung der Todesstrafe eingesetzt und Strychnin ist noch heutzutage ein Suizidgift. In der Medizin werden Reserpin (10) und Morphin (2) als Antisympathotonikum bzw. starkes Analgetikum eingesetzt. In neueren Arbeiten finden sie auch Anwendung als biogenetische oder evolutionäre Marker. ${ }^{[24]}$ Neben der pharmakologischen Wirkung spielen auch wirtschaftliche Aspekte eine entscheidende Rolle. Hierzu zählt vor allem Kaffee, welcher wohl das bedeutendste Genussmittel der Welt ist und zu 1-1.5\% das Alkaloid Coffein (1) enthält.<smiles>Cn1c(=O)c2c(ncn2C)n(C)c1=O</smiles>

Coffein 1

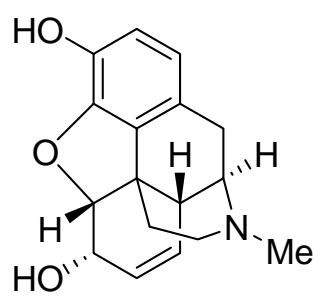

Morphin 2

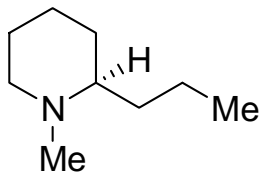

Coniin 3

Abbildung 1: Pharmakologisch aktive Alkaloide.

Da Alkaloide Sekundärmetabolite darstellen und somit nichtessentiell für den jeweiligen Organismus sind, stellt sich die Frage, was deren eigentliche Funktion ist. So wird vermutet, dass Alkaloide aufgrund des oftmals bitteren Geschmacks von Pflanzen zum Schutz vor Fraßschäden gebildet werden. Allerdings haben sich einige 
Tiere an deren Giftigkeit angepasst. So vertragen Hasen, Rehe und Kaninchen den Verzehr von Blättern der Tollkirsche, da sie über einen Entgiftungsmechanismus verfügen. Für Insekten sind diese Giftstoffe oftmals ungefährlich und können sogar im Organismus gespeichert werden, wodurch sie diesen Tieren als Selbstschutz dienen. Ferner erfüllen Alkaloide die Funktion als Pheromone, ${ }^{[25]}$ als Gifte zur Paralysierung der Opfer oder auch als Sprühreagenzien zur Abschreckung von Feinden. Als Beispiel soll das Gift von Spinnen und Wespen aufgeführt werden, welches sich aus einem komplexen Gemisch freier Aminosäuren, hochmolekularer peptidischer Toxine, einzelner Purin-Basen und kleinerer Polyamin-Toxine zusammensetzt (Abbildung 2). Aufgrund ihrer strukturellen Ähnlichkeit mit gewissen pflanzlichen Alkaloiden werden die Polyamine zu den Alkaloiden gezählt. Von den über 100.000 Spinnenarten auf der Welt wurden bisher nur 20 Spezien untersucht, demzufolge existieren nur wenige Informationen auf dem Gebiet dieser Polyamin-Toxine. ${ }^{[26]}$<smiles>NCCCNCCCCNCCC(=O)NCCCCCNC(=O)[C@H](CCCN)NC(=O)Cc1c[nH]c2cc(O)ccc12</smiles>

Abbildung 2: $\quad$ Struktur eines Polyamin-Toxins aus der Spinne.

\subsection{Klassifizierung der Alkaloide}

Alkaloide stellen eine der größten Klassen von natürlich vorkommenden Verbindungen dar. Nach groben Schätzungen konnten bisher aus pflanzlichen und tierischen Organismen oder Pilzen mehr als 10.000 Verbindungen isoliert oder nachgewiesen werden. Um sich in diesem Dschungel aus Verbindungen zurechtzufinden, ist eine Einteilung der Alkaloide in Substanzklassen notwendig.

$\mathrm{Zu}$ Beginn wurden die einzelnen Substanzen entsprechend der botanischen Klassifizierung bezeichnet, je nachdem, welcher Herkunft ihre Isolierung entsprach. Diese Herleitung begründete unter anderem die Namensgebung der Solanum- 
Alkaloide, Papaver-Alkaloide, Angostura-Alkaloide, Lobelia-Alkaloide usw. Die stetige Entwicklung und Verbreitung moderner Methoden der Strukturaufklärung, insbesondere die der NMR-Spektroskopie und Massenspektromertrie ließen eine visuelle Darstellung unbekannter Moleküle zu, was zu einer weiteren Einordnung der Alkaloide hinsichtlich ihrer strukturellen Verwandtschaft führte, zum Beispiel Pyridin-, ${ }^{[27,28]}$ Chinolin- ${ }^{[29]}$ oder Steroid-Alkaloide. Eine weitere Differenzierung dieser Verbindungsklasse wird nach deren Biosynthese vorgenommen. Das Einteilungsprinzip der Alkaloide erweist sich jedoch als sehr problematisch, da es häufig zu Überschneidungen von Merkmalen isolierter Verbindungen kommt, die ein einheitliches und somit widerspruchsfreies Einordnen nicht erlauben. In solchen Fällen kann ein Kompromiss der Klassifizierung erreicht werden, indem man als Richtlinie die Position des $N$-Atoms im Hauptstrukturelement nimmt und die Alkaloide danach unterscheidet. Daraus ergeben sich folgende Alkaloid-Klassen:

1. Heterocyclische Alkaloide

2. Alkaloide mit exocyclisch angeordneten Stickstoff-Atomen und aliphatische Amine

3. Putrescin-, Spermidin- und Spermin-Alkaloide

4. Terpen- und Steroid-Alkaloide

Die Mehrzahl der Alkaloide findet sich in der heterocyclischen Gruppe wieder, bei der das Stickstoff-Atom Teil eines Heterozyklus ist, während die restlichen drei Klassen die kleinste Einheit bilden.

\subsection{Heterocyclische Alkaloide}

\subsubsection{Indol-Alkaloide ${ }^{[30]}$}

Die Gruppe der Indol-Alkaloide umfasst ungefähr 1.500 Verbindngen. Aufgrund ihrer vielfältigen pharmakologischen Wirkung besitzen sie eine große Bedeutung. Hierzu werden Alkaloide gezählt, die sowohl das eigentliche Indol-Chromophor enthalten 
oder sich davon ableiten als auch jene, die zusätzlich einen anellierten Benzol- oder Pyridin-Ring enthalten wie Carbazole, $\beta$-und $\gamma$-Carboline und deren Derivate (Abbildung 3).<smiles>c1ccc2[nH]ccc2c1</smiles>

Indol 5<smiles>O=C1Cc2ccccc2N1</smiles>

Oxindol 6<smiles>c1ccc2c(c1)[nH]c1ccccc12</smiles>

Carbazol 7<smiles>c1ccc2c(c1)[nH]c1cnccc12</smiles>

$\beta$-Carbolin 8

Abbildung 3: Indol-Alkaloide.

Als weitere Kriterien zur Unterteilung der Indol-Alkaloide werden strukturelle und biogenetische Kriterien angewandt.

\subsubsection{Yohimban-Alkaloide ${ }^{[31]}$}

Yohimban-Alkaloide besitzen ein $\beta$-Carbolin-Grundgerüst, welches in der Natur sehr weit verbreitet ist. Diese Tatsache lässt sich vermutlich darauf zurückführen, dass desen Biogenese aus Tryptamin bzw. Tryptophan und Carbonylverbindungen relativ unkompliziert verläuft.

Die wichtigsten Vertreter dieser Gruppe sind Yohimbin (9), welches aus Pflanzen der Genera Alchornea (Euphorbiaceae), Aspidosperma, Catharanthus, Diplorrhynchus, Rauvolfia und Vinca (Apocynaceae), Corynanthe und Pausinystalia (Rubiaceae) isoliert werden kann und Reserpin (10), vorkommend in verschiedenen Alstonia-, Excavatia-, Ochrosia-, Rauvolfia-, Tonduzia-, Vallesia- und Vinca-Arten (Abbildung 4). ${ }^{[32]}$ Beide Basen existieren als verschiedene Stereoisomere, Konstitutionsisomere als auch als zahlreiche Derivate (Abbildung 4). 
<smiles>CC(=O)[C@H]1[C@@H](O)CC[C@H]2CN3CCc4c([nH]c5ccccc45)[C@H]3C[C@@H]21</smiles>

(+)-Yohimbin 9

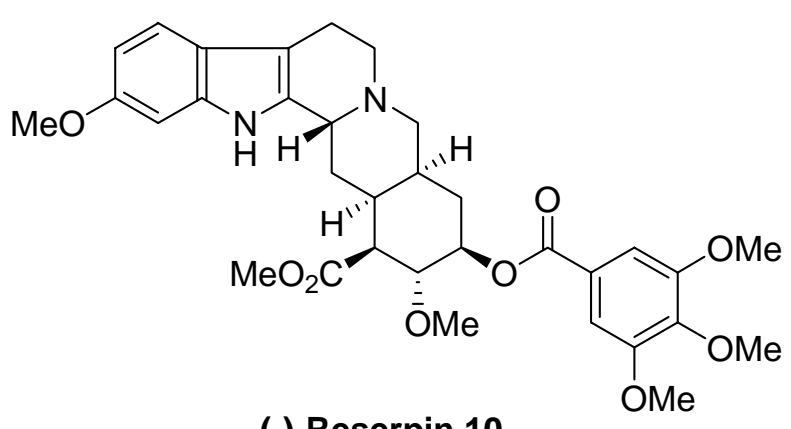

(-)-Reserpin 10

Abbildung 4: Yohimban-Alkaloide.

Yohimbin (9) wurde lange Zeit als Aphrodisiakum in der Tiermedizin genutzt, während Reserpin (10) wegen seiner hypotensiven (blutdrucksenkenden) und sedativen (beruhigenden) Wirkung eine besondere Bedeutung zukommt. ${ }^{[33]}$ Dieser Effekt beruht auf einer Senkung des Serotoninspiegels im Zentralnervensystem.

\subsubsection{Biosynthese von Yohimbin ${ }^{[34]}$}

In der Biosynthese der monoterpenoiden Indol-Alkaloide gilt das Glucosidalkaloid Strictosidin (18) als eine Schlüsselverbindung, da es sowohl die erste stickstoffhaltige Vorstufe zu den Verbindungen wie Ajmalicin und Aspidospermin als auch zu den Cinchona-Alkaloiden wie Chinin oder den Pyrrolochinolizidin-Alkaloiden, zu denen unter anderem Camptothecin (44) gehört, darstellt. Generell leiten sich die IndolAlkaloide von Tryptophan AA ab. Die Biosynthese von Yohimbin (9) verläuft ausgehend vom Tryptamin (17) und einer Dihydropyraneinheit, die sich wiederum von einem Terpen ableitet. Im ersten Schritt wird Geraniol (11) durch Oxidation zu 9Hydroxygeraniol (12) umgesetzt, dessen Umlagerung und Phosphorylierung einen Ringschluss zum Cyclopentan-System 14 erlaubt. Aus diesem Vorläufermolekül kann dann Loganin (15) aufgebaut werden, welches durch oxidative Spaltung Secologanin (16) ergibt (Abbildung 5). 


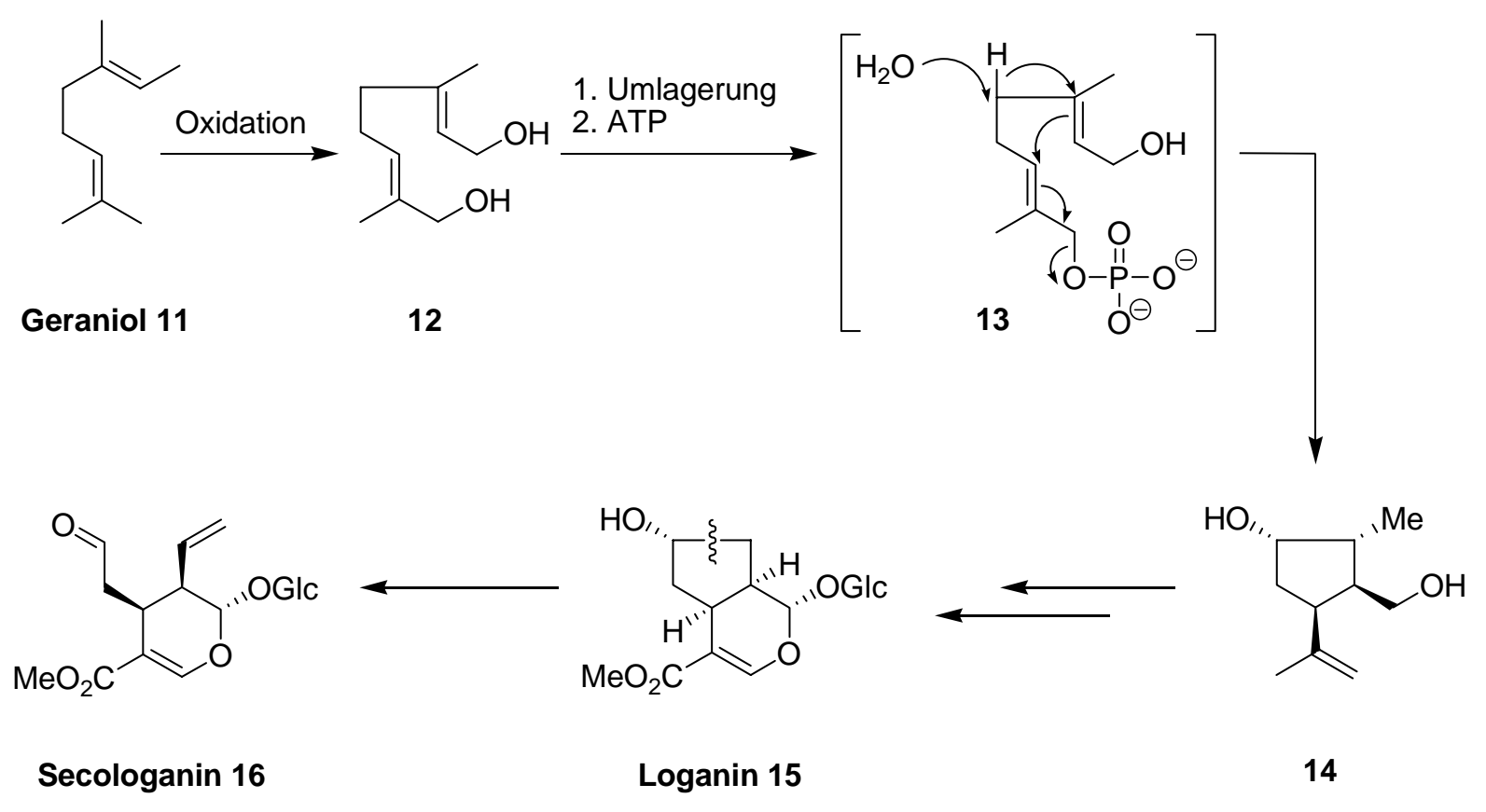

Abbildung 5: Biosynthese von Secologanin (16).

Primär wird in der Natur aus Secologanin (16) und Tryptamin (17) in einer PictetSprengler-Cyclisierung, die durch das Enzym Strictosidin-Synthase katalysiert wird, stereoselektiv das Strictosidin 18 gebildet. Das C-3 Epimer Vincosid entsteht dabei nicht. ${ }^{[35]}$ Enzymatische Deglycosidierung ergibt über das Halbacetal die acyclische Aldehydform 19, die mit dem Stickstoff am Ring zu zyklisieren vermag. ${ }^{[36]}$ Dies ergibt das entsprechende Iminiumion 20, dessen Ausbildung kinetisch kontrolliert ist und zum Corynanthe-Ringgerüst führt. Bei der thermodynamisch kontrollierten Cyclisierung des Dialdehyds 19 entsteht dagegen das vinyloge Urethan Vallesiachotamin, welches unter anderem die Vorstufe zu Antirhin und 18,19-Dihydroantirhin beschreibt.

Eine anschließende Isomerisierung von 4,21-Dehydrocorynantheinaldehyd 20 liefert das Dehydrogeissochizin 21, welches ein zentrales Intermediat weiterer IndolAlkaloide darstellt. ${ }^{[37]}$ Aus der Cyclisierung von Dehydrogeissochizin 21 resultiert letztendlich Yohimbin (9) (Abbildung 6). 


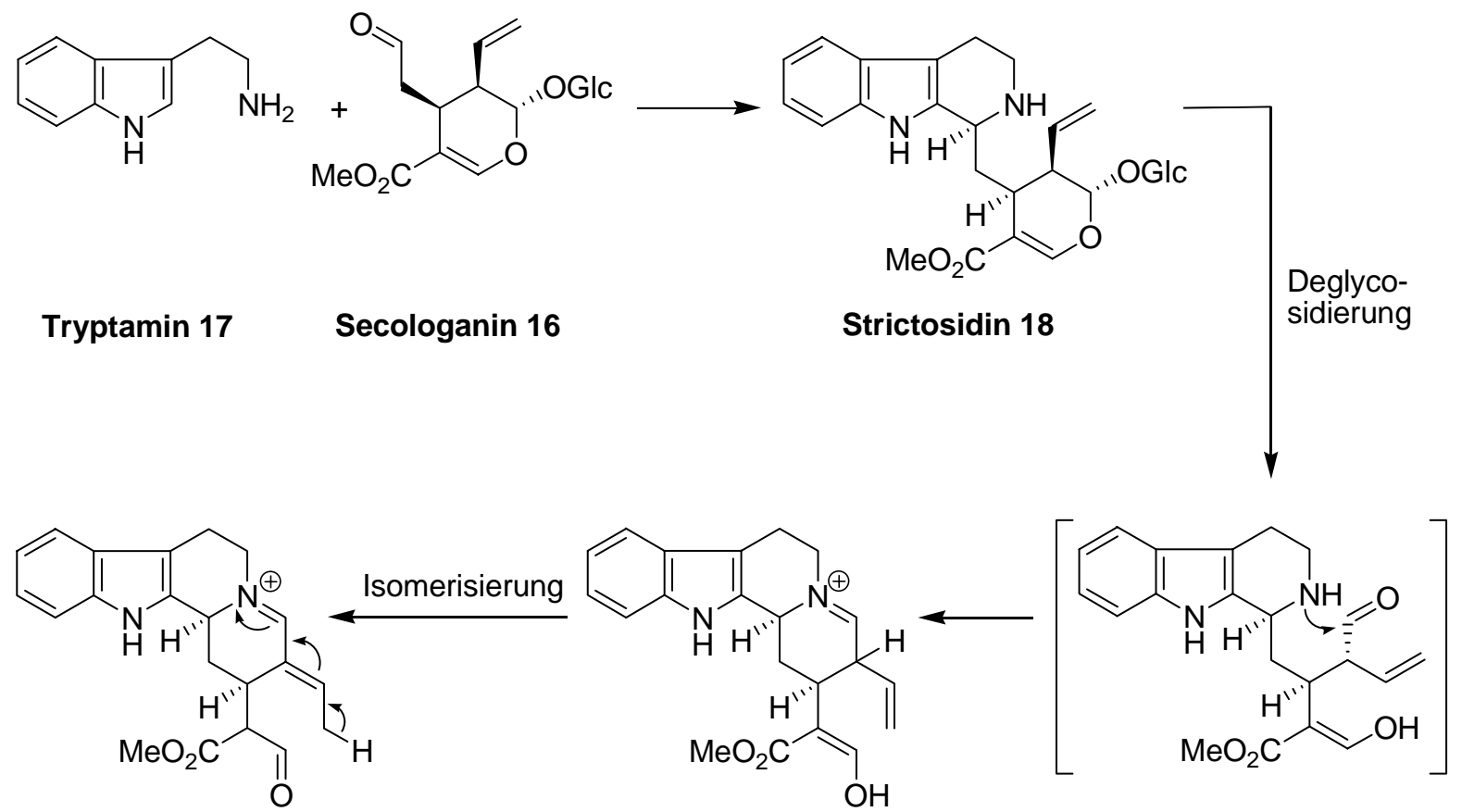

Dehydrogeissochizin 21

20

19

Aldehydform

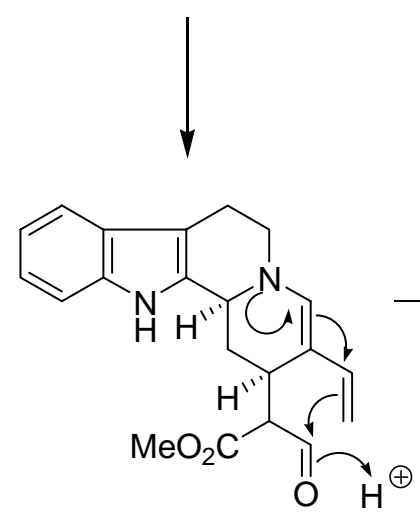

22

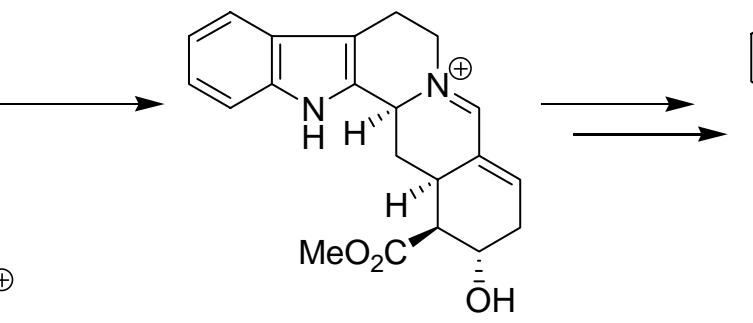

23

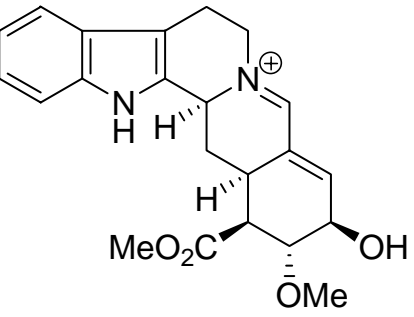

24<smiles>COC(=O)[C@H]1C[C@@H]2c3[nH]c4ccccc4c3CCN2C[C@H]1CO</smiles>

Abbildung 6: Biosynthese von Yohimbin (9). 


\subsubsection{Totalsynthese von Yohimbin $(9)^{[38]}$}

Die vielversprechende biologische Aktivität sowie die interessante pentacyclische Struktur des Yohimbins (9) wie auch seiner Strukturanaloga haben seit ihrer Entdeckung das Interesse synthetisch arbeitender Chemiker auf sich gezogen. Die historisch bedeutsame Totalsynthese von Reserpin (10) durch R. B. Woodward et al. ${ }^{[39]}$ gilt noch heute als ein Vorzeigemodell in der Strategie der präparativen organischen Chemie. Auch die erste vollständige Synthese von Yohimin (9) im Jahre 1958 von E. E. Tamelen ${ }^{[40]}$ gehört zu den beeindruckendsten Erfolgen, die in der Naturstoffsynthese erzielt wurden. Asymmetrische Darstellungen der Yohimban-Alkaloide in enantiomerenreiner Form waren jedoch für lange Zeit trotz modernster Methoden erfolglos. ${ }^{[4]}$ Erst 1986 gelang K. Szántay die Synthese der enantiomerenreinen Form von (+)-Yohimbin (9), welches aber lediglich als geringere Komponente in einem 2:1Verhältnis zu seinem C-17-Epimer isoliert wurde. ${ }^{[42]}$ Vier Jahre später publizierte T. Momose die formale Totalsynthese von (+)-Yohimbin (9), in der die stereogenen Zentren über eine asymmetrische intramolekulare Michael-Reaktion aufgebaut werden konnten. ${ }^{[43]}$ Eine erfolgreiche asymmterische Totalsynthese von (+)-Yohimbin (9) lieferte die Arbeit von J. Aubé. Für die Synthese des trans-Hydrindanons 29 wurde Butadien (25) und Fumarsäure (26) in einer asymmetrischen Diels-Alder-Reaktion zum entsprechenden Cycloaddukt 27 mit $96 \%$ de und $56 \%$ Ausbeute überführt. Die folgende Reduktion mit Lithiumaluminiumhydrid ergab ein Diol 28, welches daraufhin in drei Schritten zum gewünschten trans-Hyrindanon 29 führte. Obwohl eine Ruzicka-Cyclisierung/Decarboxylierung mit $\mathrm{BaO}$ bei $260-270{ }^{\circ} \mathrm{C}$ das Keton 29 in nur einem einzigen Schritt lieferte, wurden bessere Ergebnisse mit der drei-stufigen Sequenz, die eine Dieckmann-Cyclisierung beinhaltet, erreicht. Das isolierte transHydrindanons 29 wurde in der nächsten Stufe mit Osmiumtetraoxid umgesetzt, gefolgt von einer Acylierung, die in der Bildung von dem Diacetoxyketon 30 resultierte. Kondensation von 30 mit Tryptamin (17) und Oxidation des daraus resultierenden Imins mit mCPBA erzielte eine Mischung von zwei Isomeren, bei der das Oxaziridin 31 als Hauptprodukt mit einer Ausbeute von 67 \% hervorging. 


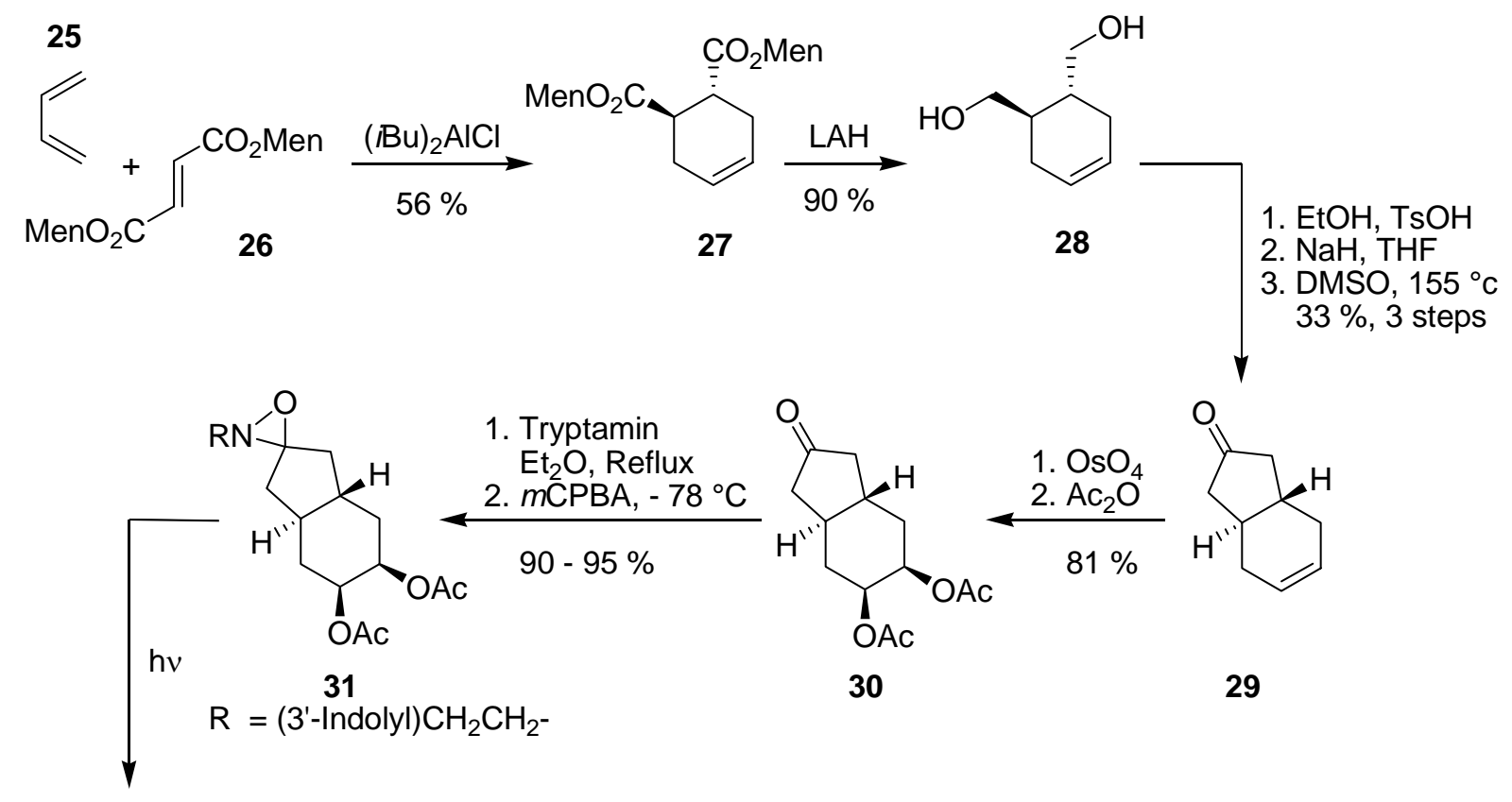<smiles>[R]C1=CC[C@H]2CC(OC(C)=O)[C@@H](OC(C)=O)C[C@H]2CC1=O</smiles>

32

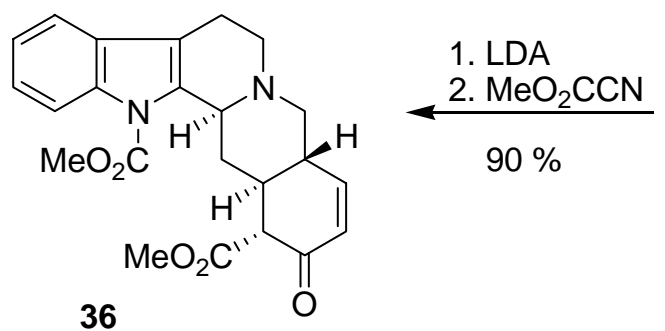

36

1. $\mathrm{H}_{2}, \mathrm{Pd} / \mathrm{C}$
2. $\mathrm{K}_{2} \mathrm{CO}_{3}, \mathrm{MeOH}$ $80 \%$

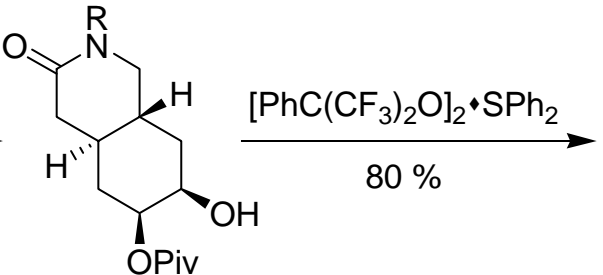

33<smiles>CO[C@@H]1C=C[C@H]2CN(CCc3c[nH]c4ccccc34)C(=O)C[C@H]2C1</smiles>

1. Bischler-

Napieralski

2. $\mathrm{NaOH}$

3. $(n \mathrm{Pr})_{4} \mathrm{NRuO}_{4}$ $48 \%, 3$ steps<smiles>O=C1C=C[C@H]2CN3CCc4c([nH]c5ccccc45)[C@H]3C[C@H]2C1</smiles><smiles></smiles> 
Anschließend wurde das Oxaziridin 31 einer Photolyse unterzogen, gefolgt von einer Hydrolye und der selektiven Veresterung der äquatorialen Hydroxygruppe mit Pivaloylchlorid. Das aus dieser Tranformation hervorgegangene Lactam 33 wurde mit Martin's Sulfuran-Reagenz umgesetzt, was eine Eliminierung der Hydroxygruppe bewirkte und somit Verbindung $\mathbf{3 4}$ zugänglich war. Die dreistufige Sequenz, bestehend aus einer Bischler-Napieralski-Cyclisierung, Hydrolyse des Pivalolylesters sowie einer Oxidation, ergab das Enon 35 in einer Ausbeute von $48 \%$. Die Anwesenheit der Enon-Funktionalität im Molekül 35 ermöglichte über eine EnolatBildung mit einem Überschuss an LDA und der anschließenden Versetzung mit Mander's Reagenz die regioselektive Einführung der Carbomethoxy-Gruppe am C-16 und somit die Bildung von 36. Es folgten die Reduktion der Doppelbindung und die selektive Deacylierung des Indol-Stickstoffs mit $\mathrm{K}_{2} \mathrm{CO}_{3}$ zum Yohimbinon (37). Im letzten Schritt führte dann die Reduktion der Keto-Gruppe zum entsprechenden Alkohol zur enantioselektiven Totalsynthese von (+)-Yohimbin (9). Zusammenfassend wurde (+)-Yohimbin (9), ausgehend von Verbindung 30, in 13 Stufen mit einer Gesamtausbeute von $7.8 \%$ erfolgreich synthetisiert (Abbildung 7).

\subsubsection{Chinolin-Alkaloide ${ }^{[44]}$}

Chinolin-Alkaloide sind seltener vertreten und kommen im Pflanzenreich vorzugsweise in Rutaceen vor. Dabei unterscheidet man unter anderem folgende Chromophore:<smiles>c1ccc2ncccc2c1</smiles>

Chinolin 39<smiles>[R]n1c(=O)ccc2ccccc21</smiles>

2-Chinolon 40<smiles>c1ccc2nc3occc3cc2c1</smiles>

Furochinolin 41<smiles>[R]n1c2ccccc2c(=O)c2ccoc21</smiles>

Furochinolon 42

Abbildung 8: Chinolin-Alkaloide. 
Neben Chinolin, das in Camelia thea, Ciutrus bigaradia und Galipea officinalis nachgewiesen wurde, findet man auch eine Vielzahl an pharmazeutisch wichtigen Chinolin-Alkaloiden. Das in der Chinarinde (Chinchona-Arten) bedeutende Chinin (43) zum Beispiel findet seine Verwendung in der Malariabehandlung, da es das Wachstum der Malariaerreger hemmt.

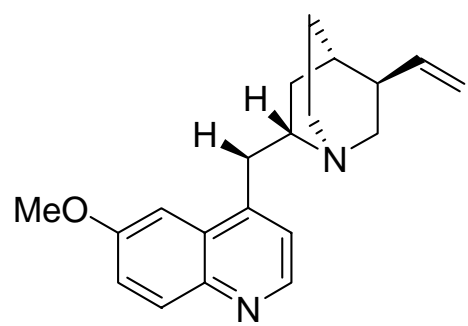

Chinin 43

Abbildung 9: Pharmazeutisch wirksames Chinin (43).

\subsubsection{Camptotheca-Basen ${ }^{[45]}$}

Hauptvertreter der Camptotheca-Basen, einer Untergruppierung der Chinolin-Alkaloide, ist Camptothecin (44), welches biogenetisch mit den Indol-Alkaloiden verwandt ist. ${ }^{[46]}$ Sein Vorkommen ist pflanzlicher Natur, vorwiegend bei Camptotheca(Nyssaceae) und Mappia-Arten (Olacaceae) und dessen biologische Herkunft auch für die Namensgebung genutzt wurde.<smiles>CC[C@H]1C(=O)OCc2c1cc1n(c2=O)Cc2cc3ccccc3nc2-1</smiles>

(+)-Camptothecin 44

Abbildung 10: Camptothecin (44). 


\subsubsection{Biosynthese von Camptothecin (44)}

Die in den Rutaceen gefundenen Chinolin-Alkaloide leiten sich von der Anthranilsäure ab. Durch Aktivierung der Carbonsäure 45 durch Coenzym A findet der nukleophile Angriff eines Moleküls Acetylcoenzym A statt. Die nachfolgende Cyclisierung zum Lacton 47 ergibt dann das Chinolingrundgerüst (Abbildung 11).

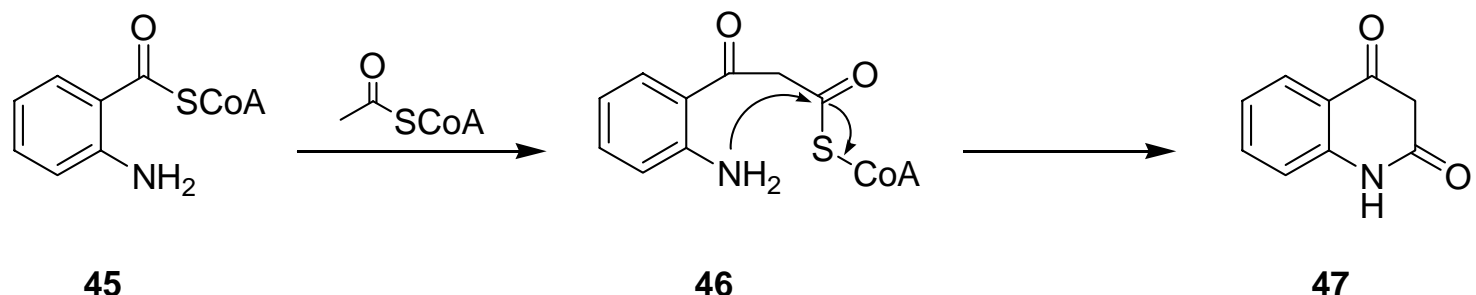

Abbildung 11: Biosynthese des Chinolingrundgerüsts 47.

\subsubsection{Totalsynthese von Camptothecin (44) ${ }^{[47]}$}

Seit der erstmaligen Isolierung von Camptothecin (44) aus der chinsesischen Pflanze Camptotheca acuminate, Nyssaceae und seiner Strukturaufklärung im Jahre 1966 von Wall et al. sorgte dieses aufgrund seiner aussergewöhnlichen Antitumoraktivität für großes Interesse in der wissenschaftlichen Gemeinschaft. ${ }^{[48,49]}$ Die anfängliche Begeisterung nahm jedoch angesichts der Probleme, die sich aus der Löslichkeit und Toxizität von Camptothecin (44) ergeben, schnell ab. Einige Jahre später führte aber die Erkenntnis, dass dieser Naturstoff eine einzigartige Wirkungsweise beruhend auf der selektiven Inhibition der DNA-Topoisomerase I besitzt, zu einem Wiederbeleben der Camptothecin-Chemie. ${ }^{[50]}$ Dies hat zu der Entwicklung von Topotecan ${ }^{[51]}$ und Irinotecan $^{[52]}$ sowie weiteren Analoga ${ }^{[53]}$ geführt, deren pharmakologische Aktivität mit der von Camptothecin (44) vergleichbar ist. Seither wurde eine Vielzahl an verschiedenen Totalsynthesen von Camptothecin (44) veröffentlicht, die auf vier unterschiedlichen Strategien aufbauen (Abbildung 12). 
56<smiles>c1ccc2nc3c(cc2c1)CNC3</smiles><smiles>CCC1(O)C(=O)OCC(C(=O)[GeH2])=C1C=O</smiles>

55<smiles>Nc1ccccc1C=O</smiles>

48<smiles>CCC1(O)C(=O)OCc2c1cc1n(c2=O)CCC1=O</smiles>

49

A

50
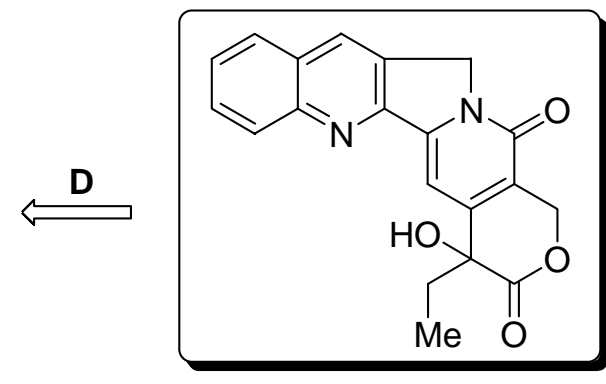

C

51<smiles>[R5]c1cc2c(c(=O)n1CC#C)C(O)(CC)C(=O)OC2</smiles>

Abbildung 12: Synthesestrategien von Camptothecin (44).

Die Synthesestrategien A und B ghören dabei zur „klassischen“ CamptothecinChemie, während die modernen Routen C und D Möglichkeiten wiederspiegeln, die die heutige Synthesetechnologie bietet.

Die folgende, racemische Totalsynthese von Camptothecin (44), publiziert von A. Kanazawa, basiert auf dem ersteren Weg. Für die Synthese des Hydroxypyridon 59 wurde die von A. Padwa entwickelte Chemie basierend auf Isomünchnon-Cyclisierungen angewendet. Dazu wurde das Diazoimid 56 in Isomünchnon 58 überführt, welches in situ eine Cycloaddition mit Methyacrylat (57) zum Hydroxypyridon 59 eingeht. Decarboxylierung mit wässrigem Bromwasserstoff ergab die Struktur 60. Die Synthese wurde mit einer Etherbildung des Hydroxypyridons 60 mit (Z)-Methyl-4chloro-2-methoxybut-2-enoat (61) und Cäsiumcarbonat fortgesetzt. 


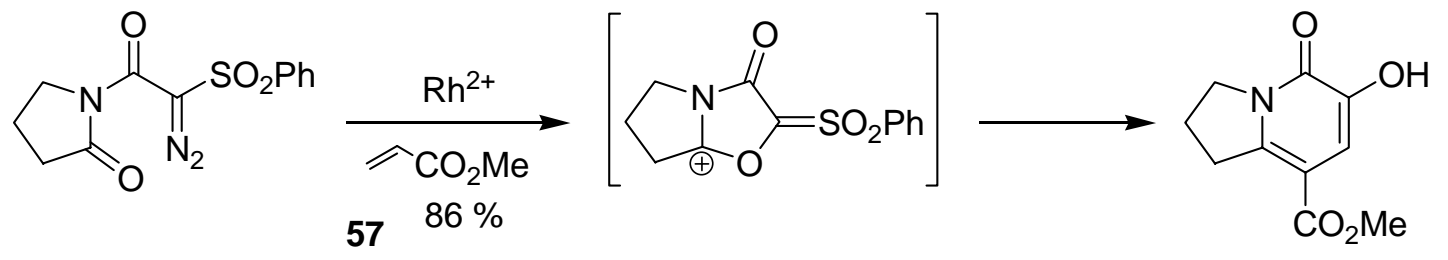

56

58

59

aq. $\mathrm{HBr}, \Delta$ $91 \%$

1. $\mathrm{Cl} \sim \mathrm{CO}_{2} \mathrm{Me}$<smiles>C=CC(C=C)(OC)c1cc2n(c(=O)c1O)CCC2</smiles>

62<smiles>O=c1c(O)ccc2n1CCC2</smiles>

60
$\mathrm{H}_{2}, \mathrm{Pd} / \mathrm{C}$

$99 \%$
$\mathrm{Cs}_{2} \mathrm{CO}_{3}$

2. PhCl, HMDS, $\triangle$<smiles>CCCC(CC)(OC)c1cc2n(c(=O)c1OC)CCC2</smiles>

64

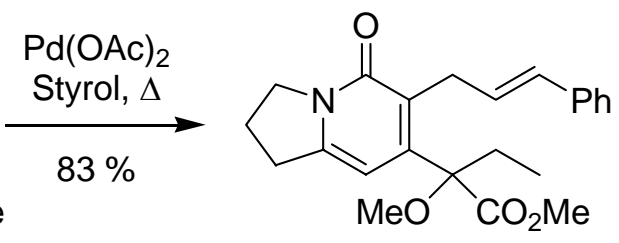

65

1. $\mathrm{O}_{3}, \mathrm{Me}_{2} \mathrm{~S}$

2. $\mathrm{NaBH}_{4}$ $80 \%, 2$ Stufen

\section{Dess-Martin \\ 2. $\mathrm{N}^{-\mathrm{Tol}}$ $\mathrm{NH}_{2} \quad 68$} $\mathrm{H}^{+}, \Delta$

$77 \%, 2$ Stufen<smiles>CCC1(OC)C(=O)OCc2c1cc1n(c2=O)CC[C@H]1O</smiles>

67<smiles>CCOS(=O)(=O)OCCCCCO</smiles>

$62 \%$<smiles></smiles>

66<smiles>CCC1(OC)C(=O)OCc2c1cc1n(c2=O)Cc2cc3ccccc3nc2-1</smiles><smiles>CCC1(O)C(=O)OCc2c1cc1n(c2=O)Cc2cc3ccccc3nc2-1</smiles>

Camptothecin 44

Abbildung 13: Totalsynthese von Camptothecin (44) nach A. Kanazawa. 
Das entstandene Derivat wurde nun einer Claisen-Umlagerung unterzogen. Die Darstellung des Hydroxypyridons 62 verlief dabei in sehr guten Ausbeuten von $60 \%$ ausgehend von Verbindung 60. Es folgten eine Hydrierung der terminalen Doppelbindung über $\mathrm{Pd} / \mathrm{C}$ zur gewünschten Verbindung 63. Die Einführung des $\alpha$ Substituenten in $\mathbf{6 5}$ gestaltete sich dagegen problematisch. Zwar gelang die Synthese des Triflat-Derivates 64, doch schlugen viele Heck-Reaktionen unter Standardbedingungen mit Styrol oder Methylacrylat anfänglich fehl. Freilich konnte das gewünschte Produkt 65 nachgewiesen werden, jedoch gelang dessen Isolierung bestenfalls mit sehr schlechten Ausbeuten. Glücklicherweise konnte diese Transformation mit hervoragender Ausbeute von $83 \%$ unter Jeffery's Konditionen verwirklicht werden. Dafür wurde Molekül 64 mit Palladiumacetat in Verbindung mit Tetrabutylammoniumacetat in Acetonitril bei $80^{\circ} \mathrm{C}$ umgesetzt. Der Lacton-Aufbau wurde vervollständigt, indem man das Styrol 65 in Anwesenheit von Pyridin bei $-100{ }^{\circ} \mathrm{C}$ mit Ozon versetzte und daraufhin der resultierende Aldehyd mit Natriumborhydrid zum Lacton 66 reduziert wurde. Der Einsatz von Seleniumoxid lieferte Verbindung 67, die im darauffolgenden Schritt mit Dess-Martin-Reagenz zum Keton reagierte. Da sich das gebildete Keton als unstabil herausstellte, wurde das Rohprodukt sofort mittels einer Friedländer-Kondensation mit einem o-Aminobenzaldehyd-Surrogat zum entsprechenden Chinolin 69 in $77 \%$ Aubeute über zwei Stufen umgesetzt. Erstaunlicherweise stellte man fest, dass Camptothecin (44) in heißem wässrigen Bromwasserstoff stabil ist, so dass auf diese Art die MethylSchutzgruppe abgespalten werden konnte und die Totalsynthese von Camptothecin (44) mit einer Ausbeute von 86 \% abgeschlossen wurde (Abbildung 13). 


\section{Theorie der Diels-Alder-Reaktion}

\subsection{Allgemeines}

Im Jahre 1928 entdeckten O.P. H. Diels und K. Alder, dass die Reaktion von pChinon (69) und Cyclopentadien (70) zu zwei Cycloaddukten $\mathbf{7 1}$ und $\mathbf{7 2}$ führt, welches noch heute als Meilenstein in der Organischen Synthese betrachtet werden kann (Abbildung 14). ${ }^{[54]}$ Die Erkenntnis, aus leicht zugänglichen Edukten mittels einer einstufigen Cycloaddition einen neuen sechsgliedrigen Ring aufbauen zu können, ließ schon damals die Schlussfolgerung zu, dass diese Methode eines der wichtigsten Werkzeuge zur Synthese von Naturstoffen mit komplizierter Molekülstruktur darstellt. Diese Annahme wurde 1950 durch die Verleihung des Nobelpreises für diese heute unter den Namen Diels-Alder-Reaktion bezeichnete Transformation bestättigt.<smiles>O=C1C=CC(=O)C=C1</smiles>

69

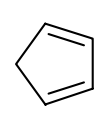

70

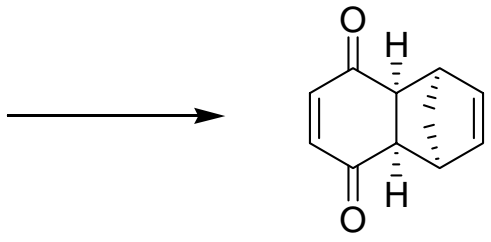

71

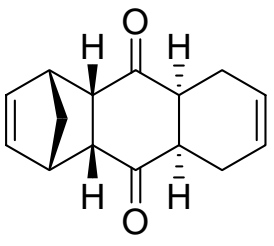

72

Abbildung 14: Historische Diels-Alder-Reaktion aus dem Jahr 1928.

Gut ein halbes Jahrhundert danach hat sich die Diels-Alder-Reaktion als eine der leistungsfähigsten Methoden in der modernen Organischen Synthesechemie etabliert und stellt ein nicht mehr wegzudenkendes Instrument in der Totalsynthese komplexer Naturstoffe dar. ${ }^{[55]}$ Die Popularität der Reaktion begründet sich zum einen in der zumeist einfachen Durchführbarkeit, zum anderen in der großen Variationsmöglichkeit hinsichtlich der einzelnen Komponenten. Desweiteren wird ihre breite Anwendbarkeit durch die Tolerierung einer Vielzahl funktioneller Gruppen gewährt, was einen leichten Zugang zu einer unbegrenzten Menge cyclischer Ringsysteme eröffnet. Oftmals ist sie das entscheidende Element einer eleganten Domino-Reaktion zur Herstellung komplizierter Molekülstrukturen. ${ }^{[56]}$ Die Reaktion verläuft konzentiert 
unter Erhalt der Stereochemie der Edukte und in den meisten Fällen regioselektiv. Es werden hierbei zwei $\sigma$-Bindungen geknüpft und somit bis zu vier stereogene Zentren aufgebaut. Die Ausbildung von zwei $\sigma$-Bindungen unter gleichzeitiger Aufgabe zweier $\pi$-Bindungen resultiert in einem beträchtlichen Energiegewinn, der die Triebkraft der Reaktion, selbst wenn ein ringgespanntes Cycloaddukt entsteht, begründet. Es konnte sogar gezeigt werden, dass die Diels-Alder-Reaktion einen synthetischen Zugang zu schwierigen Brückenkopf-Olefinen, welche die Bretsche Regel verletzen, bietet. ${ }^{[57,58]}$ Bei der Diels-Alder-Reaktion bildet sich ein einfach ungesättigter Sechsring in einer [4+2]-Cycloaddition. Das einfachste Beispiel stellt die Reaktion zwischen 1,3Butadien (25) als Dien mit Ethen (73) als Dienophil zum Cyclohexen (74) dar, die jedoch unter sehr drastischen Bedingungen abläuft (Abbildung 15).

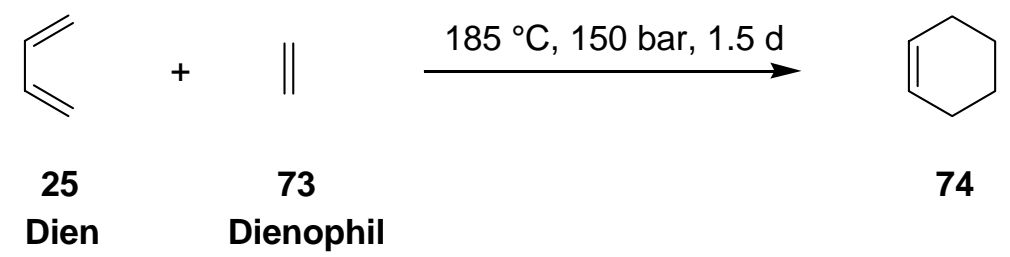

Abbildung 15: Diels-Alder-Reaktion mit 1,3-Butadien (25) mit Ethen (73).

Die Diels-Alder-Reaktion fand eine der ersteren Anwendungen in der Naturstoffsynthese in den 50iger Jahren bei der Totalsynthese von Cortison, Cholesterol und Reserpin (10) nach Woodward. ${ }^{[59]}$ Neben der wichtigen pharmakologischen Eigenschaften war es auch die komplexe Molekülarchitektur sowie die vielschichtige Funktionalisierung dieser Verbindungen, die das Interesse des organischen Synthesechemikers weckten und hierbei vor große Herausforderungen an seine Synthesestrategie stellten.Ein bemerkenswertes Beispiel einer enantioselektiven Diels-AlderReaktion zeigte kürzlich die Arbeitsgruppe von M. T. Crimmins ${ }^{[60]}$ bei der Totalsynthese der marinen und biologisch aktiven Naturstoffe Astrogorgin (78) ${ }^{[61]}$ und Ophirin B (81) ${ }^{[62]}$ (Abbildung 16 und 17).

Zunächst ergab eine vorangegangene Wittig-Olefinierung die Dienophile 76 und 75 in einem 3:1-Verhältnis. Anschließend wurde das Gemisch für mehrere Stunden bei 
Raumtemperatur stehengelassen, wobei eine intramolekulare Diels-Alder-Cycloaddition selektiv mit dem Isomer $\mathbf{7 6}$ zum gewünschten oxatricyclischen System $\mathbf{7 7}$ als einziges Diasteromer erfolgte. Das nicht umgesetzte Dienophil 75 konnte durch katalytische Mengen von PhSSPh anteilig zu Verbindung 76 isomerisiert werden und dieses wieder einer Diels-Alder-Reaktion unterzogen werden. Durch die Wiederholung dieser beiden Schritte konnte so die Ausbeute des exo-Diels-Alder-Addukts 77 auf $78 \%$ gesteigert werden (Abbildung 16).

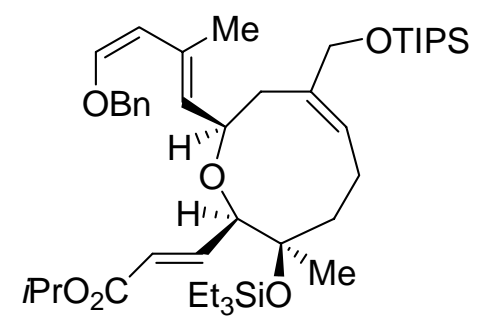

75

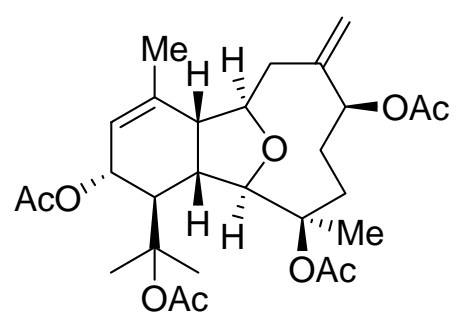

Astrogorgin 78
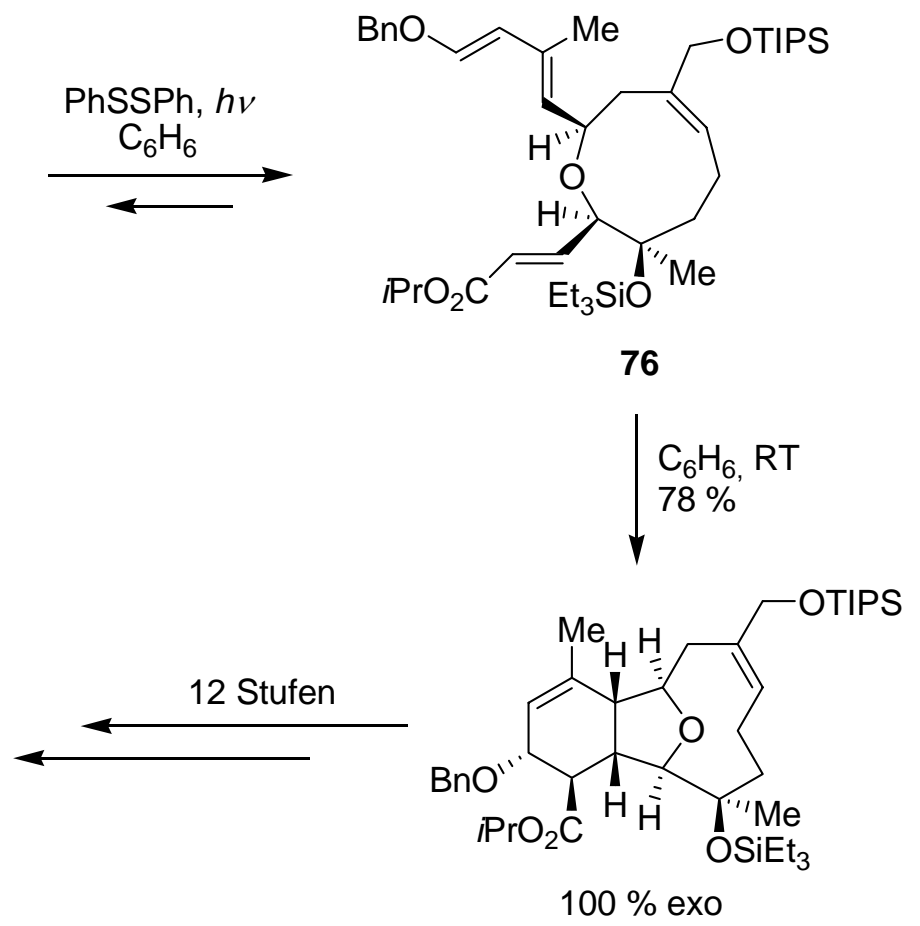

77

Abbildung 16: Intramolekulare Diels-Alder-Reaktion in Crimmins enantioselektiven Totalsynthese von Astrogorgin (78).

Auf dieselbe Art und Weise konnten auch die vier stereogene Zentren des oxatricyclischen Grundgerüsts 80 enantioselektiv über eine intramolekulare DielsAlder-Reaktion aufgebaut werden. In nur sieben weiteren Reaktionen gelang anschließend die Totalsynthese von Ophirin B (81) (Abbildung 17).

Beide Verbindungen gehören zu den Eunicellins, einer Unterklasse der C2,C11cyclisierten cembranoiden Diterpenen, welche als Sekundär-Metabolite der gorgoni- 
schen Octo-Koralle, vorkommend in der Karibik und dem westpazifischen Ozean, isoliert wurden. ${ }^{[63]}$ Seit der erstmaligen Entdeckung der Eucelline aus Eunicella Stricta im Jahre $1968^{[64]}$ führte die intensive Erforschung der gorgonischen Weich-Koralle zu einer Gewinnung von über 50 neuen Sekundär-Metaboliten dieser Klasse. Untersuchungen der biologischen Aktivität zeigten, dass diese Untergruppe neben einer Wachstumshemmung bei Insekten auch cyctotoxische Eigenschaften gegenüber verschiedenen Krebsarten aufweisen. Somit ist das Interesse, Eunicelline synthetisch zugänglich zu machen, besonders aus pharmakologischer Sicht, sehr groß.

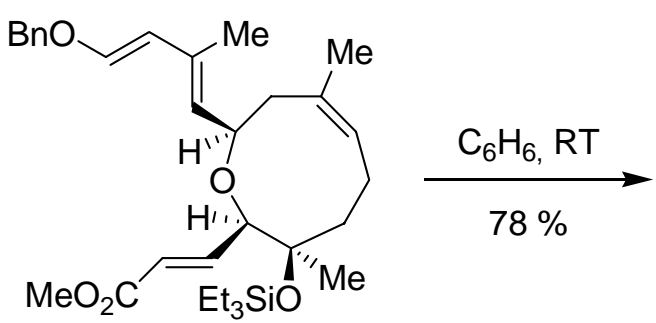

79

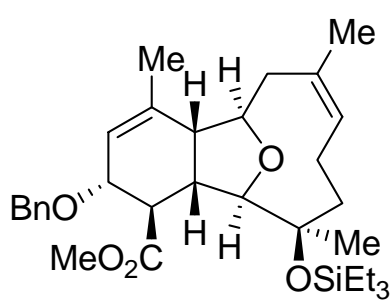

$100 \%$ exo

80

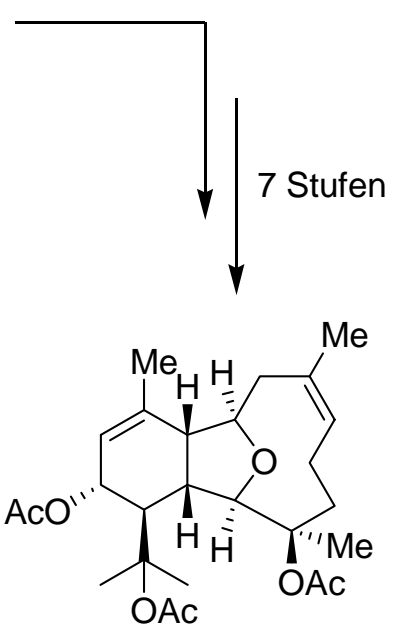

Ophirin B 81

Abbildung 17: Intramolekulare Diels-Alder-Reaktion in Crimmins enantioselektiven Totalsynthese von Ophirin B (81).

Die Strategie von A. G. Myers ${ }^{[65]}$ zum Aufbau des (-)-Tetracyclins (85), eines Antibiotikums, das seit über 50 Jahren Verwendung in der Human- als auch Veterinärmedizin findet, verkörpert ebenso einen eindrucksvolle Meilenstein innerhalb der Naturstoffsynthese. Die Besonderheit dieser Diels-Alder-Cycloaddition liegt in einem als Cyclobutan maskierten Dien (82), welches in situ durch thermischinduzierte konrotatorische elektrocyclische Ringöffnung für die Diels-Alder-Reaktion verfügbar gemacht wird. Eine direkte Umsetzung mit dem Vinylsulfid 83 unter 
lösungsmittelfreien Reaktionsbedingungen führte zu einer Ausbeute von $64 \%$ an dem hochkomplexen Tetracyclus 84, dessen weitere Transformation in nur fünf Syntheseschritten das (-)-Tetracyclin (85) lieferte (Abbildung 18).

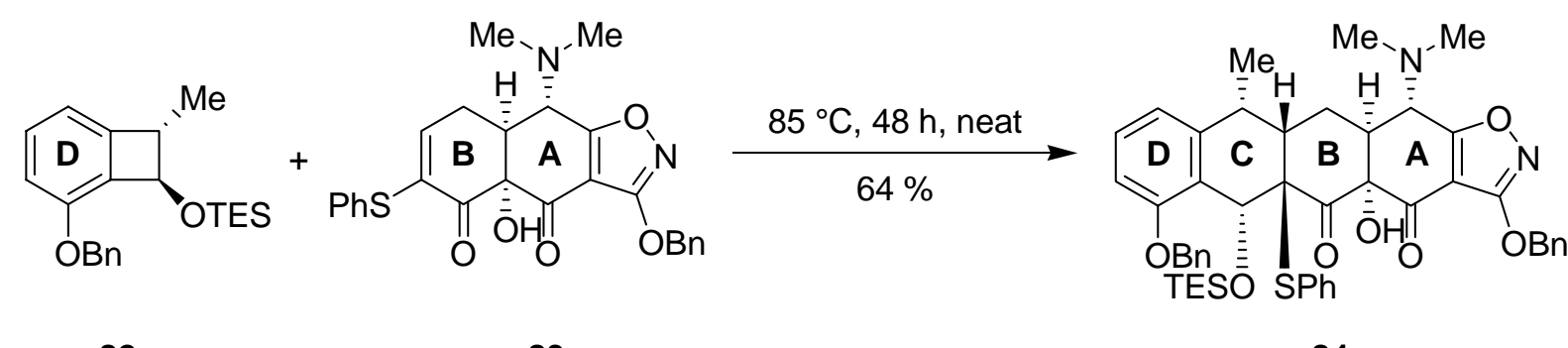

82

84

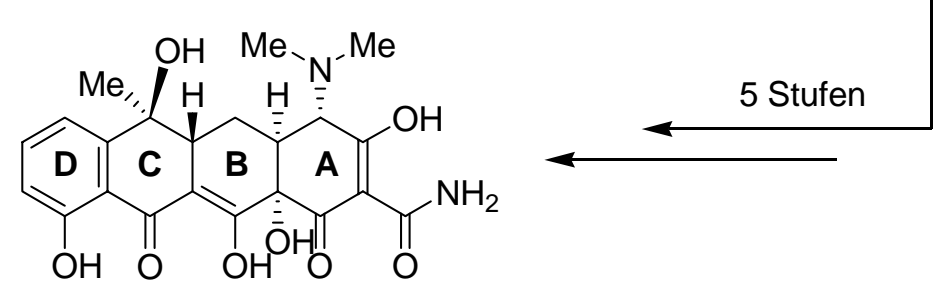

(-)-Tetracyclin 85

Abbildung 18: Diels-Alder-Reaktion unter Verwendung eines maskierten Diens 82 in der Totalsynthese von (-)-Tetracyclin (85) nach Myers.

Diels-Alder-Reaktionen beschränken sich nicht nur auf den Aufbau von Carbocyclen. Ferner ist es auch möglich, jede Position im Dien oder Dienophil von einem HeteroAtom wie Sauerstoff, Stickstoff oder Schwefel zu besetzen, wodurch sich weitere Variationsmöglichkeiten bei der Synthese von Cycloaddukten eröffnen. Man spricht dann von der sogenannten Hetero-Diels-Alder-Reaktion, die einen einfachen und effizienten Zugang $\mathrm{zu}$ sechsgliedrigen Heterocyclen erlaubt und aufgrund dieser Tatsache in der modernen Naturstoffsynthese eine besondere Stellung ein-nimmt. ${ }^{[66]}$ Der Arbeitsgruppe von A. S. Kende ${ }^{[67]}$ gelang es beispielsweise, das typischerweise aus verschiedenen Pilzen der Art Penicillium und Aspergellius isolierte Alkaloid ( \pm )Alantrypinon (90) $)^{[68]}$ über eine Hetero-Diels-Alder-Reaktion zwischen einem neuartigen, vollständig konjugierten $6 H$-Pyrazinol[2,1-b]-chinazolin-6-on 86 und 3Methylenoxindol 87 aufzubauen (Abbildung 19). Das Dien 87 konnte vorher in einer 6-stufigen Synthese, ausgehend von Anthranilsäure, in einer Ausbeute von $38 \%$ 
erhalten werden. Die Reaktion erfolgte unter sehr milden Bedingungen, wobei die Stereoselektivität durch eine bereits bekannte Bevorzugung des exo-Übergangszustandes von 2-Azadienen gesteuert wurde. Das Cycloaddukt 88 konnte so in einem exo/endo-Verhältnis von 3.14:1 und in einer Ausbeute von $73 \%$ synthetisiert werden.<smiles>CCOc1cn2c(=O)c3ccccc3nc2c(C)n1</smiles>

86<smiles>C=C1C(=O)Nc2ccccc21</smiles>

87

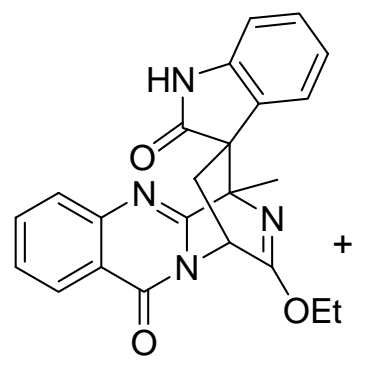

$88(55 \%)$

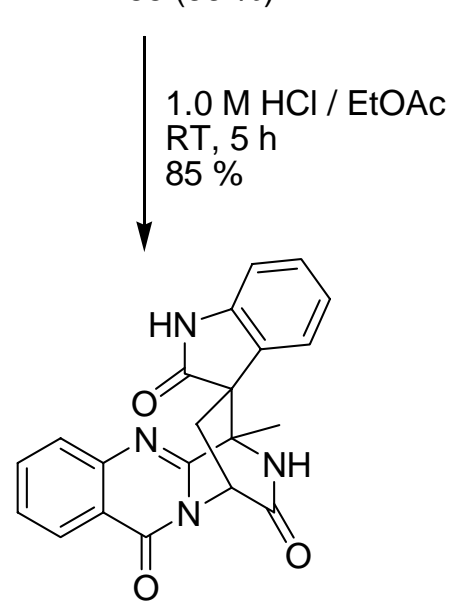<smiles>CCOC1=NC2(C=N1)CC1(C(=O)Nc3ccccc31)c1nc3ccccc3c(=O)n12</smiles>

$89(18 \%)$

(+l-)-Alantrypinon 90

Abbildung 19: Synthese von ( \pm )-Alantrypinon 90 nach Kende als Beispiel einer Hetero-Diels-Alder-Reaktion.

Der Ablauf und die Richtung der Diels-Alder-Reaktion lassen sich anhand verschiedener, empirisch gefundener Gesetzmäßigkeiten vorhersagen: ${ }^{[69]}$

1. Das cis-Prinzip: Die relative Konfiguration der Substituenten am Dien und Dienophil bleibt während der Reaktion erhalten und ist somit auch im Cycloaddukt in gleicher Form wiederzufinden.

2. Die Alder-Regel: Unterschiedlich starke elektronische Eigenschaften von Dien und Dienophil beschleunigen die Reaktion. 
3. Die endo-Regel: Bei Additionen an cyclische Diene entsteht häufig das thermodynamisch instabilere endo-Produkt im Überschuß.

4. Die ortho-Regel: Unsymmetrisch substituierte Edukte vereinigen sich regioselektiv zum ortho-Produkt.

5. Lewis-Säurekatalyse: Die Reaktionsgeschwindigkeit kann unter Verwendung von Lewissäuren gesteigert und gleichzeitig kann eine höhere Regio- und Stereoselektivität bei der Bildung des Cycloadduktes erreicht werden.

6. Lösungsmitteleffekte: Die Lösungsmittelabhängigkeit der Diels-Alder-Reaktion ist im Allgemeinen gering.

\subsection{Mechanismus}

Der detaillierte Mechanismus der Diels-Alder-Reaktion ist nach wie vor Gegenstand intensiver Forschung, wobei jedoch zumindest über bestimmte Theorien Einigkeit herrscht, die im Folgenden näher beschrieben werden sollen.

Viele mechanistische Gesetzmäßigkeiten sind schon früh, zum Teil bereits von ihren Entdeckern formuliert worden. Dennoch konnte für lange Zeit kein schlüssiger Mechanismus für deren Ablauf entwickelt werden. Ein Durchbruch wurde erst 1965 erzielt, als R. B. Woodward und R. Hoffmann das Prinzip der Erhaltung der Orbitalsymmetrie formulierten und somit erstmals die empirischen Fakten gedeutet werden konnten. ${ }^{[70]}$

Woodward und Hoffmann haben durch die Betrachtung der Symmetrie der Molekülorbitale (MOs), die durch Linearkombination der Atomorbitale eines Moleküls gebildet werden, viele Aspekte der Diels-Alder-Reaktion aufgeklärt. ${ }^{[31]} \mathrm{Sie}$ kamen dabei zu der Feststellung, dass es sich bei dieser Reaktion um eine thermisch erlaubte, orbitalkontrollierte, konzertierte [4+2]-Cycloaddition mit supra-suprafacialer Anordnug der reagierenden $\pi$-Systeme während des gesamten Reaktionsverlaufs handelt. Diese Aussage deckt sich mit der empirisch entwickelten cis-Regel.

Ebenfalls müssen Zweistufenmechanismen über ionische oder biradikalische Intermediate berücksichtig werden, da nur dann eine stereospezifische Reaktionsführung 
gewährt ist, wenn die Rotation um die neugebildete Einfachbindung langsamer ist als der Ringschluss durch Ausbildung der zweiten $\sigma$-Bindung (Abbildung 20).

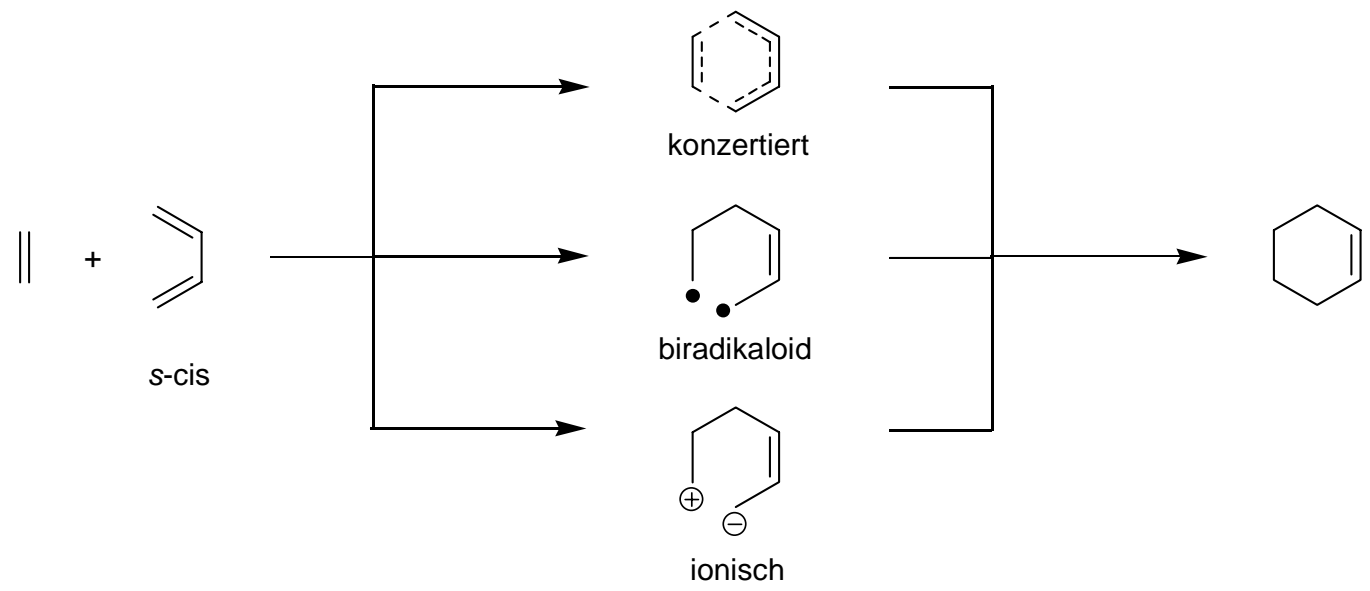

Abbildung 20: Mögliche Mechanismen der Diels-Alder-Reaktion

Ausschlaggebend für den erfolgreichen Ablauf einer Diels-Alder-Reaktion ist die s-cis-Anordnung im Dien, da ansonsten ein konzetrierter Reaktinsverlauf nicht möglich ist. Jedoch befinden sich acyclische Diene üblicherweise in der thermodynamisch stabileren s-trans-Konformation. Aufgrund der niedrigen Rotationsbarriere von ca. $30 \mathrm{~kJ} \mathrm{~mol}^{-1}$ um die zentrale $\sigma$-Bindung erfolgt aber eine schnelle Rotation zur weniger bevorzugten aber reaktiven s-cis-Konformation. Eine s-trans-Anordnung würde bei einem zweistufigen Mechanismus eine energetisch realtiv aufwendige Rotation um eine allylische Einfachbindung erfordern, sodass auf diesem Weg keine stereo-spezifische Reaktion zu erwarten ist.

Quantenmechanische $a b$ initio-Berechnungen konnten zeigen, dass bei Homo-DielsAlder-Reaktionen der konzertierte Reaktionsmechanismus im Allgemeinen energetisch günstiger ist. ${ }^{[71]}$ Ebenso deuten die stark negativen Aktivierungsentropien und Aktivierungsvolumina sowie die häufig beobachteten hohen Stereoselektivitäten darauf, dass sterisch kompakte und hochgeordnete Übergangsstrukturen ausgebildet werden, wie sie bei einem konzertierten Reaktionsverlauf anzunehmen sind. Es kann jedoch zwischen dem zweistufigen und dem konzertierten Reaktionsmechanismus Konkurrenz auftreten, wenn die gleichzeitige Bindungsbildung aus sterischen oder elektro- 
nischen Gründen erschwert ist. ${ }^{[72]}$ Im Vergleich zu Homo-Diels-Alder-Reaktionen ist bei Hetero-Dienen der Unterschied in der Aktivierungsenergie zwischen dem einstufigen, konzertierten Reaktionspfad und dem zweistufigen Mechanismus weniger stark ausgeprägt. In diesem Zusammenhang treten unsymmetrische Übergangszustände auf und in Abhängigkeit von der Natur der Substituenten ein zweistufiger Prozess, der an der reagierenden Spezies durchlaufen werden kann, was durch experimentelle Daten auch belegt werden konnte. ${ }^{[73]}$

\subsection{Die Klopman-Salem-Gleichung}

Nach dem Grenzorbitalmodell, das auf die Frontier-Molecular-Orbital-Theorie (FMOTheorie) von K. Fukui ${ }^{[74]}$ und den von K. N. Houk ${ }^{[75]}$ beschriebenen Begriff des Orbitalkoeffizienten zurückzuführen ist, hängt die Reaktivität der beteiligten Systeme im Wesentlichen von den Wechselwirkungen zwischen dem höchsten besetzten Molekülorbitas (HOMO) und dem niedrigsten unbesetzten Molekülorbital (LUMO) ab. Die bei Cycloadditionen auftretenden Übergangszustände werden elektronisch hauptsächlich durch bindende HOMO-LUMO-Wechselwirkungen stabilisiert. Der Energiegewinn $\Delta \mathrm{E}$ und die damit verbundene Stabilisierung des Übergangszustandes ist am größten, je geringer die Energiedifferenz zwischen diesen Grenzorbitalen ist. Ausgehend von der Störungstheorie haben K. Klopmann und L. Salem eine Gleichung (Gleichung 1) aufgestellt, mit derer sich die Energie $\Delta \mathrm{E}$ berechnen lässt, die bei der Überlappung der Orbitale eines Reaktanden mit denjenigen eines anderen gewonnen bzw. verloren wird. ${ }^{[76]}$

$$
\Delta \mathrm{E}=\underbrace{\sum_{\mathrm{ab}}\left(\mathrm{q}_{\mathrm{a}}+\mathrm{q}_{\mathrm{b}}\right) \beta_{\mathrm{ab}} \mathrm{S}_{\mathrm{ab}}}_{\text {1.Term }}+\underbrace{\sum_{\mathrm{k}<1} \frac{\mathrm{Q}_{\mathrm{k}} \mathrm{Q}_{1}}{\varepsilon \mathrm{R}_{\mathrm{kl}}}}_{\text {2.Term }}+\underbrace{\sum_{\mathrm{r}}^{\text {bes. }} \sum_{\mathrm{s}}^{\text {unbes. }}-\sum_{\mathrm{s}}^{\text {bes. }} \sum_{\mathrm{r}}^{\text {unbes. }} \sum_{\mathrm{r}}^{\frac{2\left(\sum_{\mathrm{ab}} \mathrm{c}_{\mathrm{ra}} \mathrm{c}_{\mathrm{sb}} \beta_{\mathrm{ab}}\right)^{2}}{\mathrm{E}_{\mathrm{r}}-\mathrm{E}_{\mathrm{s}}}}}_{\text {3.Term }}
$$

Gleichung 1: Klopman-Salem-Gleichung. 
Hierin bedeuten:

$\mathrm{q}_{\mathrm{a}}$ und $\mathrm{q}_{\mathrm{b}} \quad$ : die Elektronenpopulationen in den Atomorbitalen a und $\mathrm{b}$.

$\beta_{\mathrm{ab}}$ und $\mathrm{S}_{\mathrm{ab}} \quad$ : die Resonanz- bzw. Überlappungsintegrale der Atomorbitale $\mathrm{a}$ und $\mathrm{b}$.

$\mathrm{Q}_{\mathrm{k}}$ und $\mathrm{Q}_{1} \quad$ : die Gesamtladung an den Atomen a und $\mathrm{b}$.

$\varepsilon \quad$ : die lokale Dielektrizitätskonstante.

$\mathrm{R}_{\mathrm{kl}} \quad$ : den Abstand zwischen den Atomen a und $\mathrm{b}$.

$\mathrm{c}_{\mathrm{ra}} \quad:$ den Koeffizienten des Atomorbitals a im Molekülorbital r.

$\mathrm{c}_{\mathrm{sb}} \quad:$ den Koeffizienten des Atomorbitals b im Molekülorbital s.

$\mathrm{E}_{\mathrm{r}}$ und $\mathrm{E}_{\mathrm{s}} \quad$ : die Energien der Molekülorbitale $\mathrm{r}$ und $\mathrm{s}$.

Die drei verschiedenen Terme repräsentieren dabei die unterschiedlichen Arten von Wechselwirkungen der sich annährenden Reaktanden. Der erste Term, die sogenannte closed-shell-Abstoßung, beschreibt die antibindenen Wechselwirkungen der besetzten Molekülorbitale der miteinander reagierenden Moleküle. Da es bei der Annäherung zweier Reaktionspartner in jedem Fall zu einem Energieanstieg kommt, beinhaltet dieser Term den größten Anteil der Aktivierungsenergie der Reaktion. Im zweiten Term werden die elektrostatischen Coulomb-Wechselwirkungen der Moleküle betrachtet, die vor allem bei ionischen oder stark polaren Spezies von Bedeutung sind. Term 3 veranschaulicht die Wechselwirkungen aller besetzten mit allen unbesetzten Molekülorbitalen korrekter Symmetrie (bindende Wechselwirkung). Er ist für die Reaktion zwischen unpolaren Reaktionspartnern, wie sie normalerweise bei DielsAlder-Reaktionen auftreten, am wichtigsten und führt in jedem Fall zu einer Energieabsenkung.

Durch einige Nährungen lässt sich die Klopman-Salem-Gleichung erheblich vereinfachen. So kann man annehmen, dass die closed-shell-Abstoßung bei ähnlichen oder verwandten Reaktionswegen in etwa identisch ist und somit vernachlässigt werden kann. Außerdem kann, sofern es sich nicht um polare oder ionische Verbindungen handelt, auch der zweite Term unberücksichtigt bleiben. Bei näherer Betrachtung des dritten Term wird deutlich, dass die Beiträge energetisch sehr 
unterschiedlicher Molekülorbitale vernachlässigbar klein sind $\left(E_{r}-E_{s}\right.$ wird groß und steht im Nenner). Den größten Beitrag zu Term 3 liefern somit die energetisch am dichtesten zusammenliegenden Orbitalpaare, die HOMOs und LUMOs der Reaktionspartner. Nach Fukui ist demnach eine Beschränkung auf die Betrachtung der sogenannten Grenzorbitale zulässig. Bei der Diels-Alder-Reaktion werden nur die Wechselwirkungen zwischen den endständigen Atomen 1 des Diens mit 1' des Dienophils und 4 des Diens mit 2' des Dienophils betrachtet (Abbildung 21).

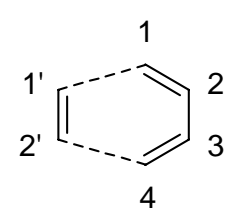

Abbildung 21: Wechselwirkende Atome bei der Diels-Alder-Reaktion.

Aufgrund dieser Näherungen vereinfacht sich die Klopman-Salem-Gleichung auf folgende Weise (Gleichung 2):

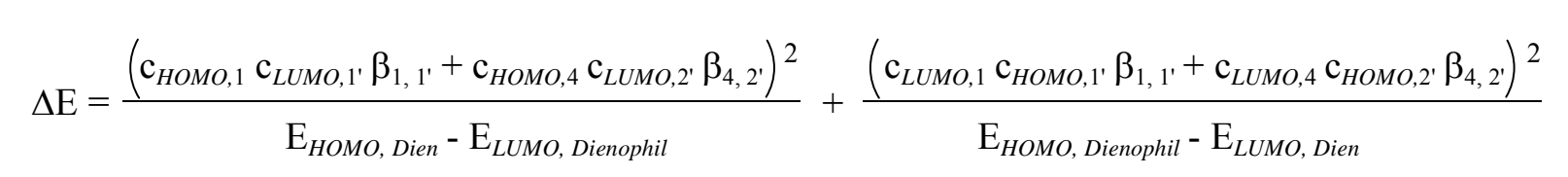

Gleichung 2: Vereinfachte Klopman-Salem-Gleichung.

Aus der Klopman-Salem-Gleichung geht hervor, dass der Energiegewinn $\Delta \mathrm{E}$ und somit die Reaktivität einer Diels-Alder-Reaktion umso höher ist, je geringer die Energiedifferenz zwischen dem HOMO und dem LUMO der jeweiligen Reaktanden ist. Vereinfachend ist, dass sich die Orbitalenergien im Gegensatz zu den Übergangsstrukturen leicht experimentell bzw. quantenmechanisch bestimmen lassen. So entspricht die HOMO-Energie ca. dem negativen Wert des ersten Ionisierungspotentials und ist demzufolge durch Photoelektronenspektroskopie ermittelbar. ${ }^{[77]}$ LUMOEnergien sind aus polarographisch bestimmbaren Redoxpotentialen zugänglich. ${ }^{[78]}$ 


\subsection{Einteilung der Diels-Alder-Reaktionen}

Aus der Klopman-Salem-Gleichung geht hervor, dass der Energiegewinn und damit die Reaktivität einer Reaktion umso größer sind, je geringer die Energiedifferenz zwischen dem HOMO und dem LUMO der einzelnen Reaktanden ist. Ein Ziel sollte daher sein, durch geeignete Substituenten der Reaktionspartner diese Energiedifferenz so weit wie möglich zu minimieren. Zum Erreichen einer Reaktivitätssteigerung muß daher die HOMO-Energie durch elektronenliefernde Substituenten angehoben und die LUMO-Energie durch elektronenziehende Substituenten abgesenkt werden. Je nach energetischer Lage der betrachteten Grenzorbitale lässt sich die Diels-Alder-Reaktion in drei verschiedenen Typen unterteilen (Abbildung 22) ${ }^{[39]}$ Typ I wird als Diels-AlderReaktion mit normalem Elektronenbedarf bezeichnet. Bei diesem Typ dominiert die Wechselwirkung zwischen dem HOMO des Diens und dem LUMO des Dienophils den energetischen Ablauf der Reaktion. Elektronenliefernde Substituenten im Dien bewirken eine Anhebung der HOMO-Energie und Elektronenakzeptoren im Dienophil eine Absenkung der LUMO-Energie. Dadurch rücken die Energien der Grenzorbitale näher zusammen und bewirken eine erhöhte Reaktivität. Folglich laufen Diels-AlderReaktionen mit normalem Elektronenbedarf zwischen elektronenreichen Dienen und elektronenarmen Dienophilen bevorzugt ab. Bei dem zweiten Typ handelt es sich um die so genannte neutrale Diels-Alder-Reaktion, welche sich durch ähnliche oder gleiche Orbitalenergien beider Reaktanden auszeichnet. In diesem Fall müssen beide Möglichkeiten der HOMO-LUMO-Wechselwirkungen berücksichtigt werden. Die Einführung jeglicher Art von Substituenten hat zur Folge, dass die Energiedifferenz HOMO-LUMO verringert wird und somit eine Reaktivitätssteigerung bewirkt wird. Diels-Alder-Reaktionen mit neutralem Elektronenbedarf laufen im Allgemeinen nur unter drastischen Bedingungen wie hohe Temperaturen, hoher Druck oder lange Reaktionszeiten ab (vgl. Abbildung 15). 


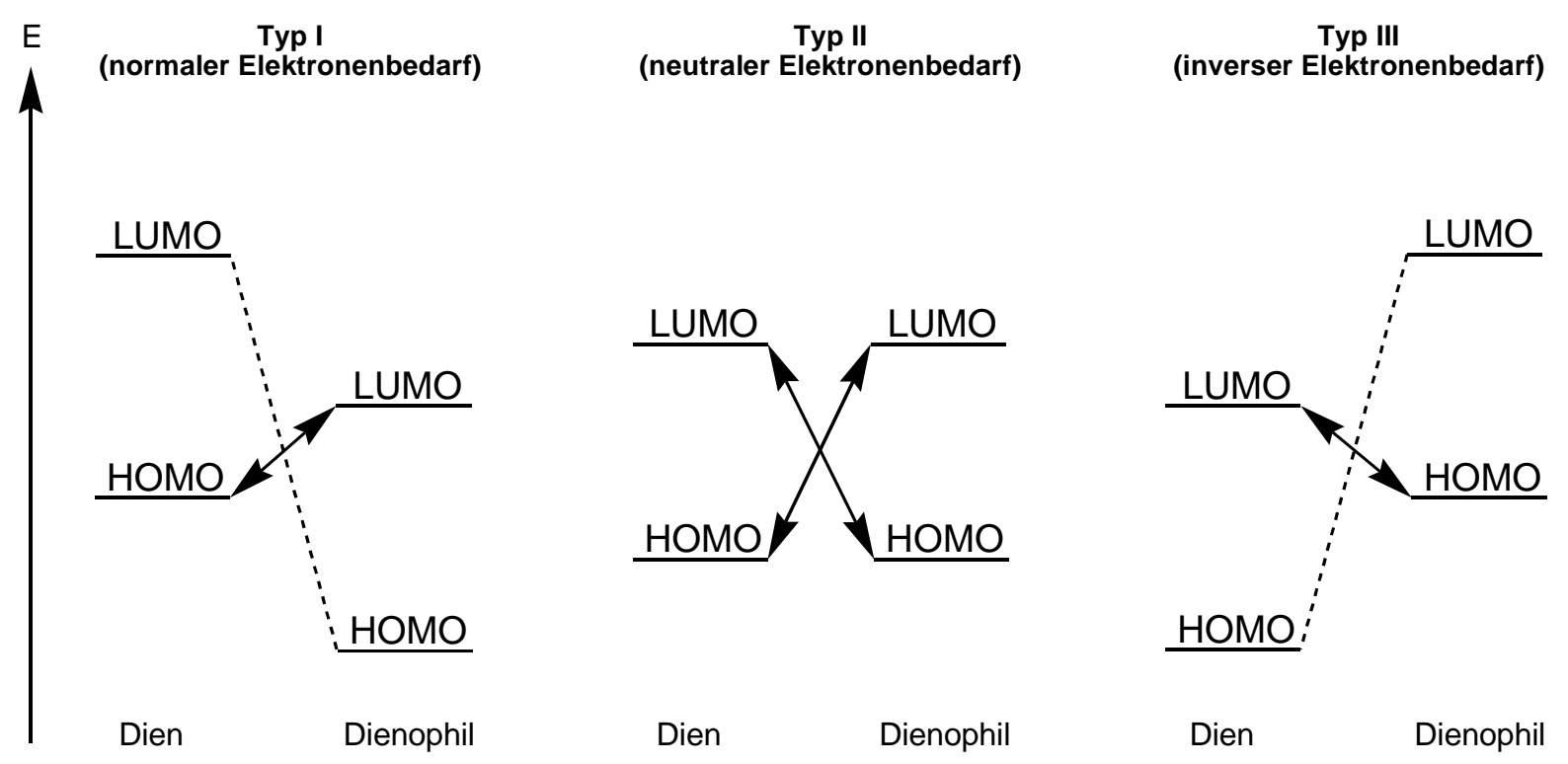

schematisch:
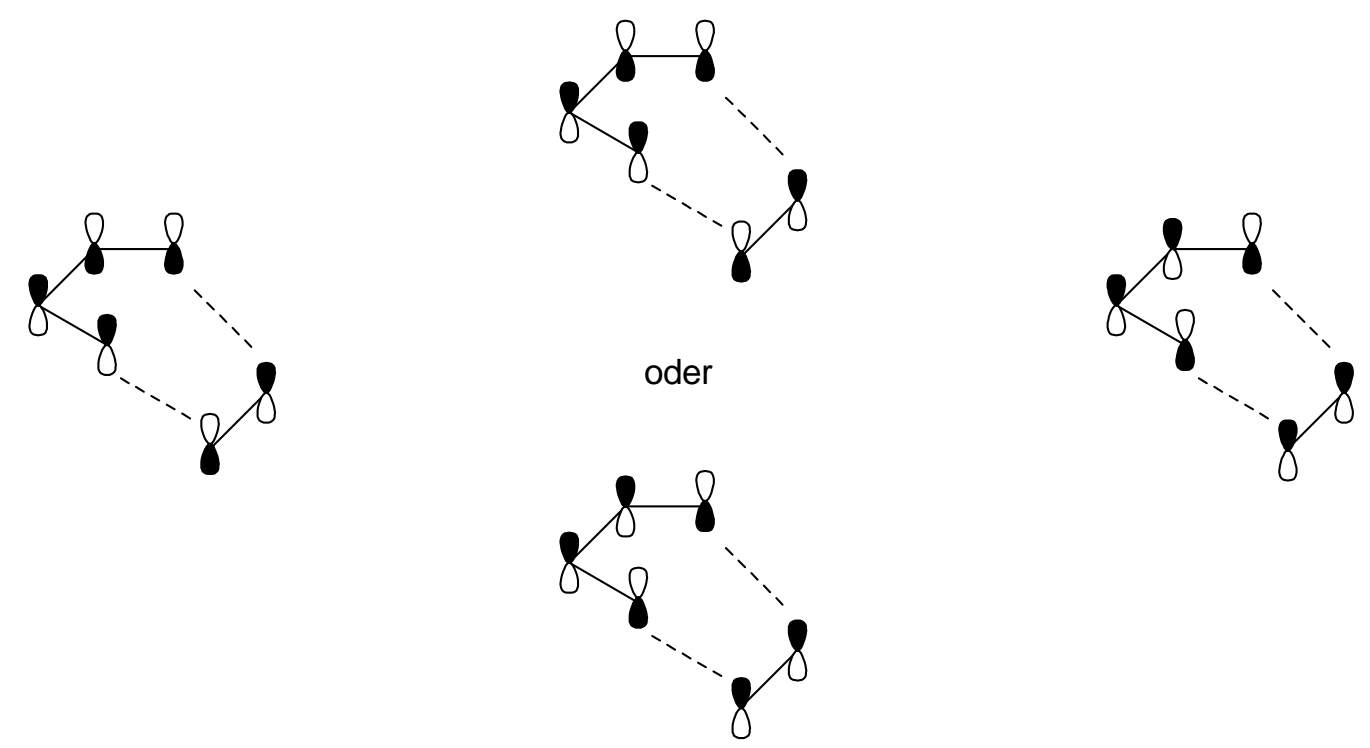

Typ II steht die inverse Diels-Alder-Reaktion (Typ III) gegenüber. Hier dominiert die Wechselwirkung zwischen dem LUMO des Diens mit dem HOMO des Dienophils. Elektronenziehende Substituenten im Dien und Elektronendonatoren im Dienophil senken die Differenz der Grenzorbitalenergien und bewirken so eine Reaktionsbeschleunigung. 
Beispiel:

normal<smiles>C=CC=COC=CC=O</smiles><smiles>CCOC1CCCC[C@@H]1C=O</smiles>

neutral

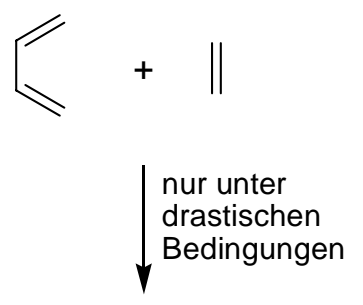

1 invers<smiles>C=CC=[N+](C)COCC</smiles><smiles>CCO[C@@H]1CCC=CO1</smiles>

Abbildung 23: Einteilung der Diels-Alder-Reaktionen nach ihrem Elektronenbedarf.

Diese Einteilung erlaubt eine qualitative Voraussage über das Reaktivitätsmuster einer Diels-Alder-Reaktion. Eine Klassifizierung setzt die Kenntnis der Beeinflussung der energetischen Lage der Grenzorbitale durch Substituenteneffekte voraus. Mit diesem Problem setzte sich K. N. Houk auseinander und schlug eine Gliederung der Substituenten in drei Klassen vor. ${ }^{[79]}$

1. C-Substituenten: Zur Konjugation befähigte Systeme wie Phenyl- oder Vinylgruppen. Sie senken die LUMO-Energie und heben die HOMO-Energie an.

2. Z-Substituenten: Elektronenakzeptoren, z.B. Nitro-, Carbonyl- oder Cyanogruppen, senken sowohl die HOMO- als auch die LUMO-Energie.

3. X-Substituenten: Elektronendonatoren, wie Amino-, Alkyl- oder Alkoxygruppen, heben die HOMO- und LUMO-Energie an.

Aus dieser Gruppierung geht hervor, dass C-Substituenten generell aktivierend auf eine Diels-Alder-Reaktion wirken, während bei einer Umsetzung mit normalem Elektronenbedarf Z-Substituenten am Dienophil und X-Substituenten am Dien diese beschleunigt wird. Diels-Alder-Reaktionen mit inversem Elektronenbedarf verlaufen 
dagegen deutlich schneller, wenn sich X-Substituenten am Dienophil und Z-Substituenten am Dien befinden.

\subsection{Regioselektivität}

Die Grenzorbitaltheorie bietet nicht nur eine gute Grundlage zum Verständnis der Reaktivität einer Diels-Alder-Reaktion, sie ermöglicht auch eine Erklärung der Regioselektivität bei einer Transformation zwischen unsymmetrischen Edukten. Diese wird durch die Molekülorbital-Koeffizienten der miteinander reagierenden Atome bestimmt, welche durch ESR-spektroskopische Messungen und qunatenmechanische Rechnungen (PM3-Methode) ermittelt werden können.

Abbildung 24 zeigt die unterschiedliche Beeinflussung der HOMO-LUMO-Energien und der MO-Koeffizientengröße beim Dien und Dienophil in Abhängigkeit vom Substituententyp. Für die häufig auftretende Diels-Alder-Reaktion mit normalem Elektornenbedarf kann man die Wirkung der Substituenten auf die Orbitalkoeffizienten wie folgt zusammenfassen:

Im HOMO eines 1,3-Diens ist der MO-Koeffizient

1. an C-4 umso größer im Vergleich zu dem an C-1, je stärker der elektronengebende Effekt eines Substituenten an C-1 ist;

2. an C-1 umso größer im Vergleich zu dem an C-4, je stärker ein an C-2 gebundener Donor ist;

3. an C-4 größer als an C-1, wenn sowohl C-2 als auch C-1 denselben Donor-Substituenten besitzen. 


\section{Olefine:}

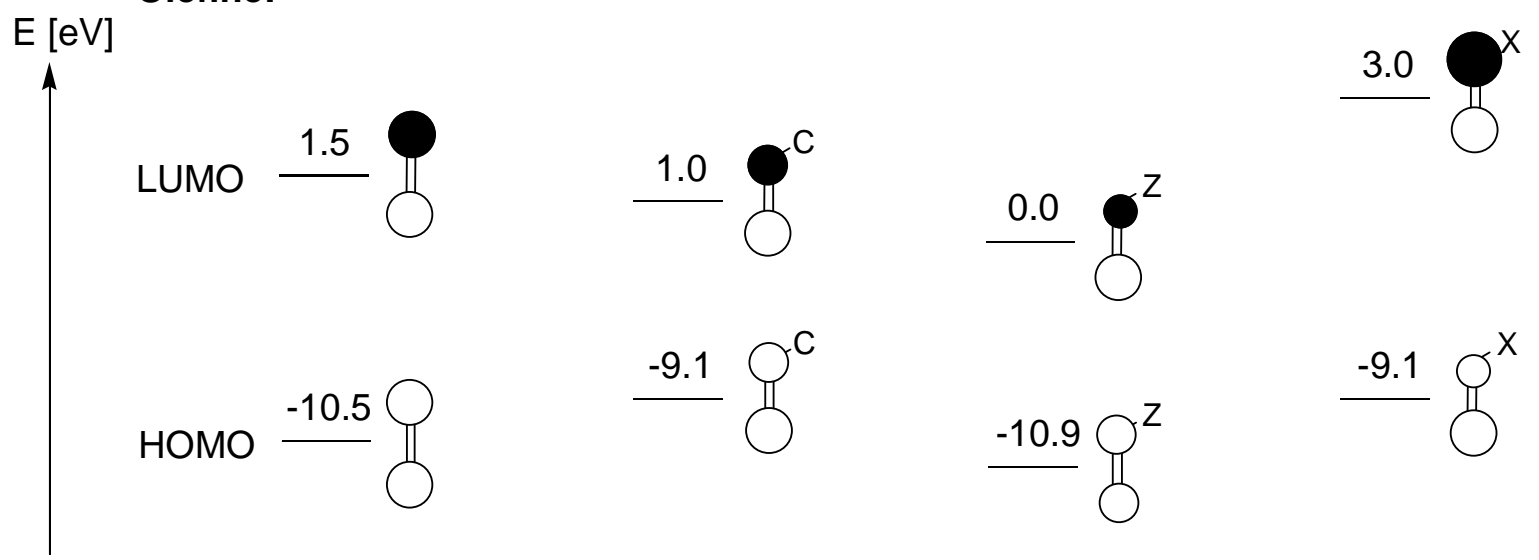

\section{1-substituierte Diene:}

$\mathrm{E}[\mathrm{eV}]$

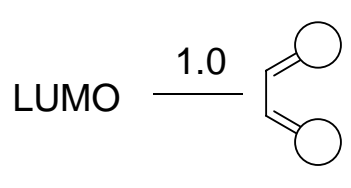<smiles>CO/C(C)=C/C=S</smiles><smiles>[X]C(C)=CC(C)=O</smiles>

HOMO $\stackrel{-9.1}{0}$
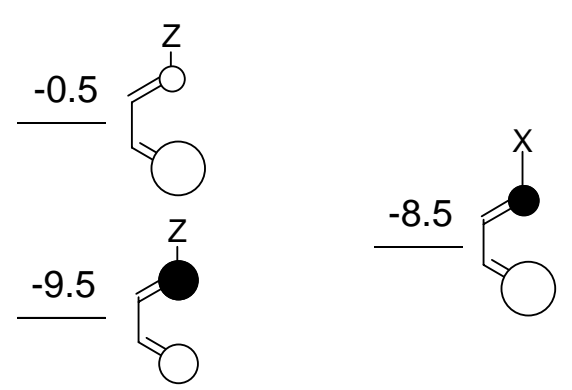

2-substituierte Diene:

$\mathrm{E}[\mathrm{eV}]$<smiles>COC(=O)C(=O)OCC(=O)CO</smiles><smiles>O=CC=C(C=O)C=O</smiles><smiles>C=CC(C=O)=CCC</smiles><smiles>[X]C(=O)C=O</smiles>

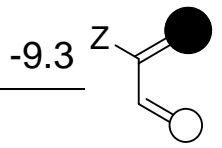<smiles>CCC(C)=CC=O</smiles>

Abbildung 24: Einfluß unterschiedlicher Substituenten auf die Orbitalkoeffizienten und Grenzorbitalenergien nach K. N. Houk. 
Im LUMO eines Dienophils ist der MO-Koeffizient

1. an C-2 umso größer im Vergleich zu dem an C-1, je höher die Anzahl elektonenziehnder Substiutenten an C-1 ist;

2. an C-2 umso größer als an C-1, je stärker der an C-1 gebundene Akzeptor ist.

Eine bindende Überlappung zweier großer bzw. kleiner Orbitalkoeffizienten ist gegenüber der eines kleinen mit einem großen Koeffizienten bevorzugt (Abbildung 25). Dieser Sachverhalt wird im Zähler des dritten Terms der Klopman-Salem-Gleichung qunatifiziert: der Energiegewinn $\Delta \mathrm{E}$ wird dann hoch, wenn das Produkt aus den MOKoeffizienten der wechselwirkenden Zentren entsprechend groß ist. ${ }^{[80]}$

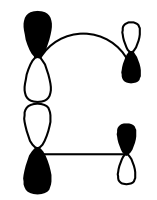

bevorzugt

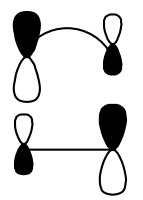

nicht bevorzugt

\section{Abbildung 25: Atomorbitalüberlappung.}

Die Reaktion des methoxysubstituierten Diens 92 mit dem Acrylsäuremethylester 57 veranschaulicht beispielhaft einen regioselektiven Verlauf einer Diels-Alder-Reaktion.<smiles>CC=CCCC</smiles>

91<smiles>COC=CCOC(C)=O</smiles>

HOMO<smiles>C=C/C=C\OC</smiles>

92<smiles>C=CC(=O)OC</smiles>

57<smiles>COC(=O)[C@H]1CCC=C[C@H]1OC</smiles>

93

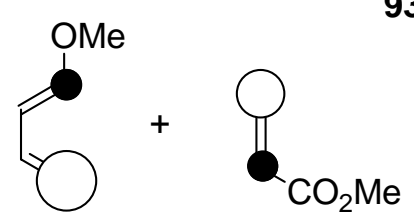

HOMO LUMO

Abbildung 26: Steuerung der Regioselektivität durch MO-Koeffizienten. 


\title{
3.6. Stereoselektivität
}

Die Stereochemie der Diels-Alder-Produkte ist von den unterschiedlichen Geometrien, die beim Übergangszustand auftreten können, abhängig.

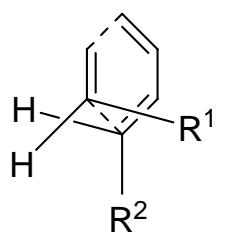

endo-E-syn

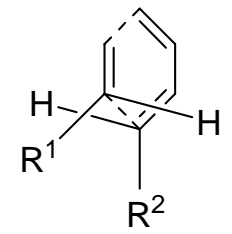

exo-E-anti
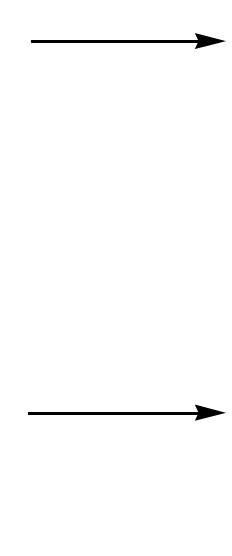

(1)

\begin{abstract}
Abbildung 27: Mögliche Übergangsstrukturen intermolekularer Diels-Alder-Reaktionen.
\end{abstract}
Die Annäherung des Dienophils ist generell aus zwei verschiedenen Richtungen möglich (Abbildung 27). Orientiert sich der Substituent des Dienophils in Richtung des Diens, so wird dies als endo-Angriff bezeichnet. Bei der entgegengesetzten Konstellation, bei der der Substituent des Dienophils dem Dien abgewandt ist, wird vom exo-Angriff gesprochen. Bei mehrfach substituierten Dienophilen greifen die Cahn-Ingold-Prelog-Regeln (CIP-Regeln), die Bezeichnung richtet sich demzufolge nach dem Substituenten mit der höchsten Priorität. Hingegen wird bei Hetero-DielsAlder-Reaktionen die Stellung des Substituenten mit der höchsten Priorität am Dienophil zum Heteroatom des Diens betrachtet. Die Orientierung des Dienophils im Übergangszustand und die Doppelbindungskonfiguration des Diens bestimmen hingegen die relative Konfiguration des Cycloaddukts.

Bei intramolekularen Diels-Alder-Reaktionen spielt die Orientierung der Kette von Atomen, durch die Dien und Dienophil miteinander verbunden sind, die tragende Rolle. Liegt die verbindende Kette ober- oder unterhalb der Ebene des Dienophils, so 
wird der Übergangszustand als endo bezeichnet. Von einem exo-Übergangszustand (Abbildung 28) spricht man hingegen, wenn sich die Kette außerhalb der Dienebene befindet.

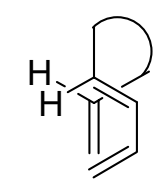

endo-E-syn

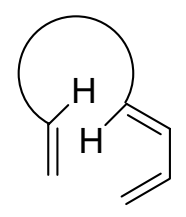

exo-E-anti

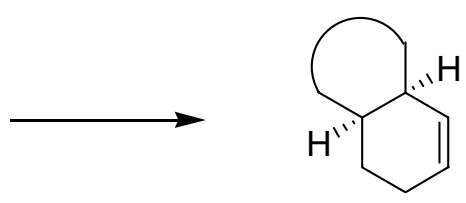

cis-Cycloaddukt<smiles>C1=C[C@H]2CCCCC[C@H]2CC1</smiles>

trans-Cycloaddukt

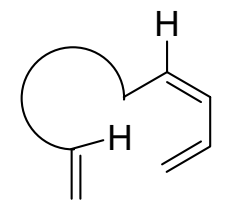

exo-Z-syn

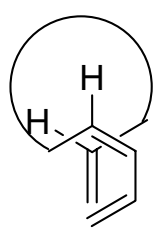

endo-Z-anti

Abbildung 28: Mögliche Übergangsstrukturen intramolekularer Diels-Alder-Reaktionen.

Im Gegensatz zur Regioselektivität, die die FMO-Theorie treffend beschreibt, konnte bislang das häufige Auftreten der endo-Selektivität nicht bis ins letzte Detail aufgeklärt werden. So wurden lange Zeit stabilisierende, nichtbindende sekundäre Orbitalwechselwirkungen für das Zustandekommen der endo-Selektivtät zur Begründung herangezogen. Es wurde angenommen, dass der sogenannte „SecondaryOverlap“-Effekt eine Herabsenkung der Aktivierungsenergie des endo-Übergangszustandes bewirkt, welcher dadurch dem exo-Übergangszustand zu bevorzugen sei. ${ }^{[81]}$ Berechnungen konnten jedoch zeigen, dass die Stabilisierung durch diesen Effekt weniger als $0.3 \%$ der benötigten Aktivierungsenergie beträgt und somit keine ausreichende Erklärung für die endo-Selektivität darstellt. ${ }^{[82]}$ Neure Untersuchungen stützen eher die Annahme, dass die endo-Selektivität durch sterische Ursachen, Lösungsmitteleffekte oder eventuell auftretende „Wasserstoff-Bindungen“ ausgelöst wird. $^{[83]}$ 


\section{Domino-Reaktionen}

\subsection{Das Konzept der Domino-Reaktion}

Die Anforderungen, den die heutige moderne, organische Synthesechemie gerecht werden soll, werden immer größer. Ziel ist es nicht mehr allein, die oftmals von der Natur vorgegebenen komplexen Molekülarchitekturen nachzubauen. Vielmehr wird gerade im industriellen Bereich versucht, sich den gesellschaftlichen Bedürfnissen nach immer größeren Mengen an chemisch gefertigten Erzeugnissen anzupassen. Dies erfordert einen wohlüberlegten Umgang mit vorhandenen Rohstoffen und lässt somit ökologische und ökonomische Aspekte immer mehr in den Mittelpunkt rücken. Ein wegweisendes Konzept, das diese Ansprüche erfüllt, sind sogenannte DominoReaktionen. ${ }^{[84]}$ Nach L. F. Tietze definiert man damit einen Prozess, bei dem zwei oder mehrere Bindungsknüpfungen, üblicherweise C-C-Bindungen, oder Fragmentierungen unter identischen Reaktionsbedingungen stattfinden, wobei die nachfolgende Transformation an der im vorangegangenen Schritt gebildeten Funktionalität erfolgt. Solche Reaktionen zeichnen sich häufig durch große Eleganz, hohe Stereoselektivität und einfache Reaktionsführung aus und ermöglichen es, komplexe Moleküle in wenigen Stufen aufzubauen. Die Entwicklung derartiger Synthesemethoden erlaubt darüber hinaus die Minimierung unerwünschter Abfallprodukte durch das Einschränken von Aufarbeitungs- und Reinigungsschritten.

Domino-Reaktionen sind in der Natur häufig vertreten, insbesondere bei der Biosynthese von Alkaloiden, Terpenen und Steroiden. Ein faszinierendes Beispiel ist die Cyclisierung des Squalenoxids 94 zum Lanosterin (95), einem Steroidvorläufer (Abbildung 29). ${ }^{[85] \mathrm{i}}$ Dabei laufen diese Reaktionen in der Natur allerdings über die Multienzymkomplexe der Zelle ab, was eine synthetische Nachahmung im Labor erschwert. 


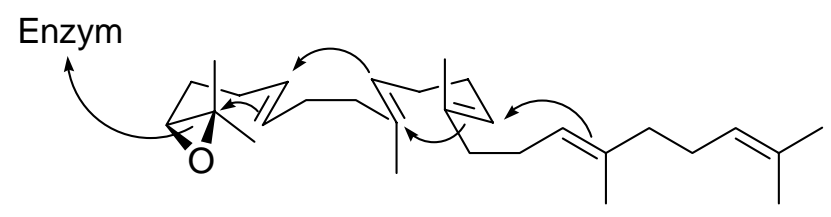

94

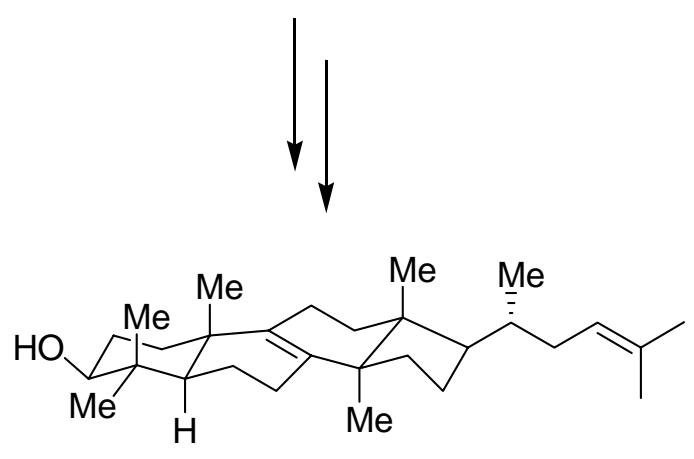

Lanosterin 95

Abbildung 29: Biosynthese von Lanosterin (95).

Nach L.F. Tietze werden die einzelnen Transformationen Reaktionskategorien zugeordnet und die Dominoprozesse durch Art und Reihenfolge der vollzogenen Schritte beschrieben (Tabelle 1).

\section{Transformation}

kationisch

anionisch

radikalisch

pericyclisch

photochemisch

carbenoid

übergangsmetallinduziert

oxidiert/reduziert

enzymatisch

\section{Transformation}

kationisch, anionisch, radikalisch oder pericyclisch anionisch, kationisch, radikalisch oder pericyclisch radikalisch, kationisch, anionisch oder pericyclisch pericyclisch, kationisch, anionisch oder radikalisch anionisch oder radikalisch

Tabelle 1: Klassifizierung von Domino-Prozessen nach L. F. Tietze. 
Als Hauptkriterium der schematischen Klassifizierung wurde die reaktive Spezies Kation, Anion oder Radikal - gewählt, die generell in einer einleitenden Reaktion gebildet wird. Bei den meisten Domino-Prozessen verlaufen die beiden ersten Transformationen über den gleichen Typ, wobei die anionisch-anionischen Reaktionen am häufigsten vertreten sind. Bei vielen Domino-Reaktionen erfolgen zudem die zweiten und nachfolgenden Schritte intramolekular. Eine Vielzahl der möglichen Permutationen ist bereits bekannt. Doch läßt diese Art von Reaktionsführung soviel Raum, dass sie aktueller Gegenstand intensiver Forschung vieler Arbeitsgruppen ist.

\subsection{Domino-Reaktionen in der organischen Synthese}

Ein aktuelles Beispiel für die Effizienz der biomimetischen Domino-Reaktionen ist die von E. J. Sorensen entwickelte Totalsynthese von (+)-FR182877, ${ }^{[86]}$ bei der zwei transannulare Diels-Alder-Reaktionen zum Aufbau des polycyclischen Grundgerüstes verwendet wurden (Abbildung 30).

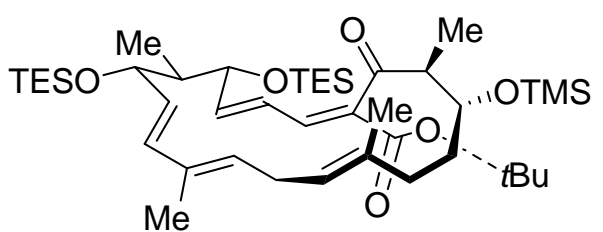

96

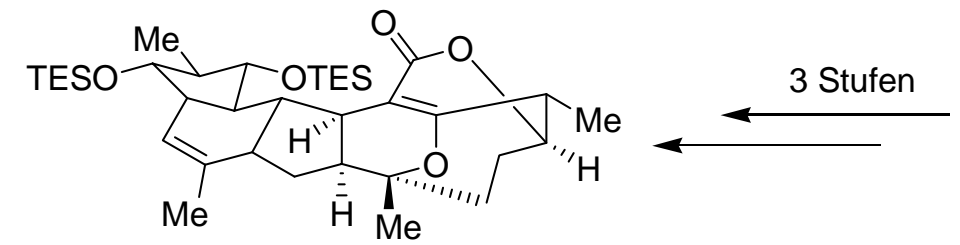

(+)-FR182877 (99)

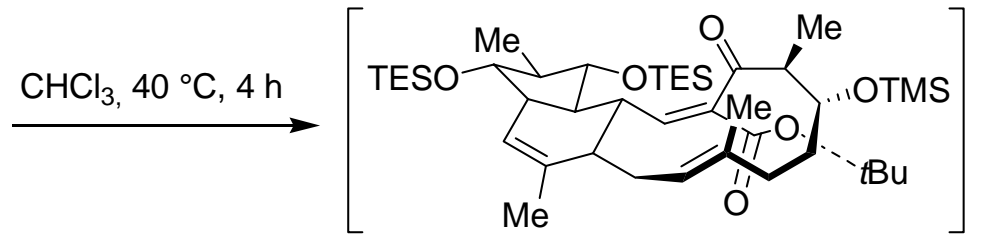

97 $40 \%$ $\downarrow$

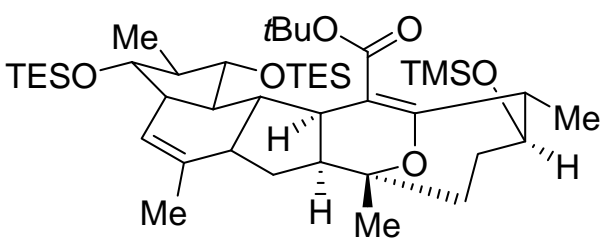

98

Abbildung 30: Totalsynthese von (+)-FR182877 (99) über eine doppelte transannulare Diels-AlderReaktionskaskade nach E. J. Sorensen. 
Eine breite Anwendung in der Naturstoffsynthese fand auch die im Arbeitskreis von

L. F. Tietze entwickelte Domino-Pictet-Spengler-En-Reaktion, ${ }^{[87]}$ Domino-Knoevenagel-En-Reaktion $^{[88]}$ und Domino-Knoevenagel-Hetero-Diels-Alder-Reak-tion. ${ }^{[89]}$ Durch die Domino-Knoevenagel-En-Reaktion als hochstereoselektive Synthesemethode für trans-1,2-disubstituierte Cyclohexane und Cyclopentane konnte in einer enantioselektiven Synthese das Sesquiterpen Veticadinol erhalten werden. ${ }^{[90]}$ Die Knoevenagel-Reaktion von Dimethylmalonat (100) mit dem eine En-Komponente enthaltenden Aldehyd 101 ergab das Dien 102, das in der anschließenden En-Reaktion zum trans-1,2-disubstituierten Cyclohexan 103 abreagierte.

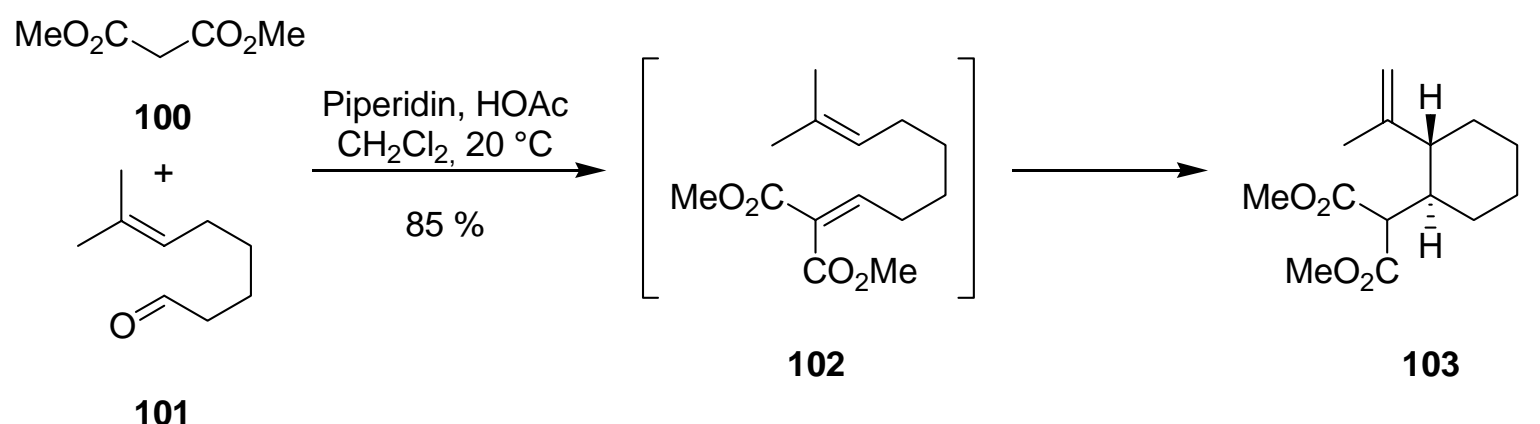

Abbildung 31: Domino-Knoevenagel-En-Reaktion.

\subsubsection{Domino-Knoevenagel-Hetero-Diels-Alder-Reaktion}

Die intensive Auseinandersetzung mit Domino-Reaktionen in den letzten Jahren hat insbesondere die Domino-Knoevenagel-hetero-Diels-Alder-Reaktion als ein potentes Werkzeug zur Synthese von komplexen Molekülstrukturen wie Naturstoffen in den Vordergrund gebracht. ${ }^{[91]}$

Sie stellt eine Kombination aus einer Knoevenagel-Reaktion ${ }^{[92]}$ einer 1,3Dicarbonylverbindung mit einem Aldehyd in Gegenwart einer schwachen Base wie EDDA oder Piperidiniumacetat und einer anschließenden hetero-Diels-AlderReaktion $^{[93]}$ mit einem Enolether, Enamin oder alkylsubstituierten Alken dar. Die hetero-Diels-Alder-Reaktion des zuvor in der Kondensationsreaktion gebildeten 1- 
Oxa-1,3-butadiens verläuft mit inversem Elektronenbedarf. Das bedeutet, dass die Wechselwirkung zwischen dem LUMO des Diens mit dem HOMO des Dienophils dominiert. Die Vielseitigkeit dieser sequentiellen Transformation ist vor allem darin begründet, dass sowohl cyclische Substrate wie Meldrum-Säure, BarbitursäureDerivate und Dimedon als auch Cumarine und $\beta$-Ketoester sowie ihre Phosphor-, Stickstoff- und Schwefel-Analoga als 1,3-Dicarbonylenolate fungieren können. Desweiteren ist eine Reaktionsführung als zwei, drei oder vier Komponenten-Reaktion möglich. ${ }^{[94]}$

Ein beeindruckendes Beispiel einer Domino-Knoevenagel-hetero-Diels-AlderReaktion spiegelt sich in der von L.F. Tietze ${ }^{[95]}$ entwickelten enantioselektiven Totalsynthese von dem Ipecacuanha-Alkaloids Emetin (110), einem Antiprotozoikum, wieder. Die Kondensation von Aldehyd 104 mit Meldrum's-Säure (106) liefert zunächst das Intermediat 107, welches in situ mit dem anwesenden Enolether 105 in einer Hetero-Diels-Alder-Reaktion zu dem nicht isolierten Cycloaddukt 108 weiter reagierte. Abschließende Fragmentierung unter Freisetzung von $\mathrm{CO}_{2}$ und Aceton ergab das Zielprodukt 109 zusammen mit zwei Diastereomeren im Verhältnis von 1.5:1.0:1.8 in $86 \%$ Ausbeute. Ausgehend von 109 konnte in nur vier weiteren Stufen Emetin (110) synthetisch bereitgestellt werden (Abbildung 32).

Aufbauend auf der Domino Knoevenagel-hetero-Diels-Alder-Reaktionssequenz wurde darüber hinaus ein Konzept der stereochemischen Kombinatorik entwickelt, das den einfachen und effizienten Aufbau einer Bibliothek von 12 Stereoisomeren des Ipecacuanha-Alkaloids Emetin (110) ermöglichte (Abbildung 32). 


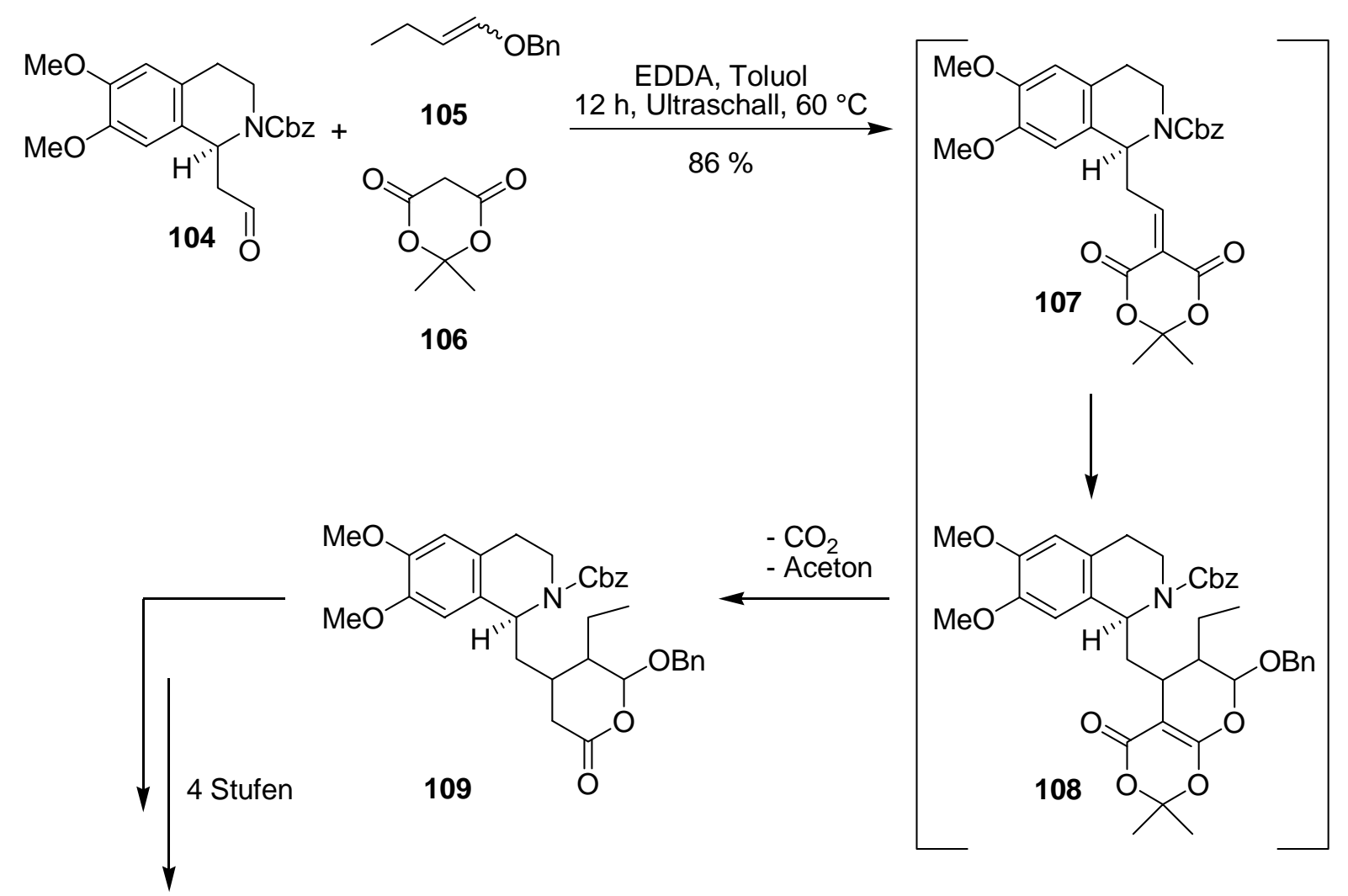<smiles>CC[C@H]1CN2CCc3cc(OC)c(OC)cc3[C@@H]2CC1C[C@@H]1NCCc2cc(OC)c(OC)cc21</smiles>

Abbildung 32: Domino Knoevenagel-hetero-Diels-Alder-Reaktionssequenz zur Darstellung von Emetin (110). 


\section{Problemstellung und Planung der Arbeit}

\section{$5.1 \quad$ Einleitung}

Viele Alkaloide, zu denen vornehmlich die aus Pflanzen isolierten Verbindungen zählen.sind durch biologisch eindrucksvolle Wirkungsweisen gekennzeichnet. Deren breiter Anwendungsbereich wird vor allem durch den Einsatz in vielen Arzneimitteln deutlich. Gemäss dem International Drug Directory von 1995 wurden unter anderem folgende Alkaloide aufgeführt, deren Nutzen für den Menschen außer Frage steht: Ajmalin mit antiadrenerger, in hohen Dosen auch sedativer Wirkung, und Spartein werden zur Behandlung von Arrhytmien eingesetzt. Codein, ein Antitussivum, das bedeutet ein den Hustenreflex hemmendes Mittel, weist auch opioide Eigenschaften auf, auch wenn diese geringer ausfallen als bei Morphin. Weitere Beispiele sind das Antimigränemittel Dihydroergotamin, Ergotamin als Wehenmittel sowie Chinin, welches als Antipyretikum und als Antimalaria-Medikament genutzt wird. Hervorzuheben sind auch diejenigen Alkaloide, die bei der Behandlung von Krebserkrankungen ihren Nutzen finden, so wie Vinblastin, Vincristin, Leurosidin und Leurosin. Diese vier Verbindungen konnten aus einer einzigen Pflanze extrahiert werden. Die Behandlung mit dem dimeren Indolalkaloid Vincristin hat dazu geführt, dass bei akuter Leukämie von Kindern, die früher tödlich war, eine Heilungschance von über $70 \%$ erreicht wird. Eine beachtliche Menge der natürlichen Alkaloide wird dabei weiterhin aus Pflanzen direkt gewonnen, was sich als problematisch gestaltet, weil deren Anteil aus diesen Quellen oftmals sehr gering und der Aufwand der Isolierung groß ist. Aber gerade diese Gegebenheiten verdeutlichen das langanhaltende synthetische, pharmakologische und nicht zuletzt auch das finanzielle Interesse an effizienten Wegen zum Aufbau dieser Strukturen. Voraussetzung zur Nutzung dieses großen pharmakologischen Potentials sind daher kurze Synthesen, welche einen schnellen und variablen Zugang zu eben diesen Molekülstrukturen ermöglichen. Einer noch wichtigeren Bedeutung kommt jedoch der Verwendung von Alkaloid-Analoga als so genannte „Lead“-Substanzen zu. Hierbei handelt es sich um StrukturModifikationen, die dieselbe oder auch eine unterschiedliche Wirkung wie ihre 
Vorbilder besitzen. So erlauben oft diese Derivate unerwünschte Begleiterscheinungen der alkaloidischen Substanzen zu beseitigen oder soweit wie möglich diese zumindest zu verringern. Moderne Verfahren, die dem hohen Anspruch der Naturstoffsynthese gerecht werden, sind oft Domino-Reaktionen. Diese zeichnen sich im Allgemeinen durch Ressourcenschonung, Abfallvermeidung und eine hohe Bindungseffizienz aus. Die im Arbeitskreis von L. F. Tietze entwickelte Domino-Knoevenagel-hetero-DielsAlder-Reaktion mit einer darauf folgenden Cyclisierung stellt einen effizienten, ökonomisch und ökologisch sinnvollen Zugang zu monoterpenoiden Indolalkaloiden der Vallesiachotamin- und Corynanthe-Gruppe als auch der Isochinolinalkaloiden vom Ipecacuanha-Typs dar. Auf diese Weise konnten unter anderem Hirsutin 111, Dihydrocorynanthein 112, Dihydroantirhin 113, Emetin 110 und Tubulosin 114 in enantiomerenreiner Form synthetisch zugänglich gemacht werden (Abbildung 33).

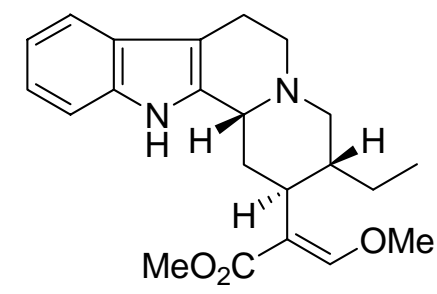

Hirsutin 111

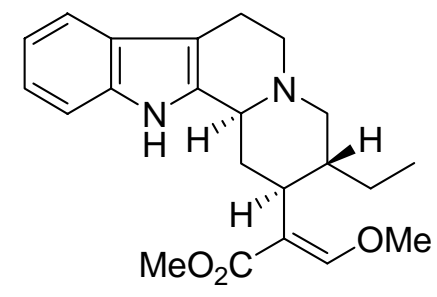

Dihydrocorynanthein 112

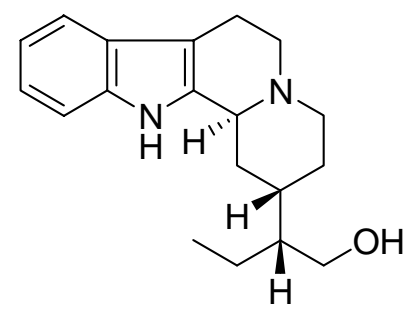

Dihydroantirhin 113<smiles>CC[C@H]1CN2CCc3cc(OC)c(OC)cc3[C@H]2C[C@H]1C[C@@H]1NCCc2cc(OC)c(OC)cc21</smiles>

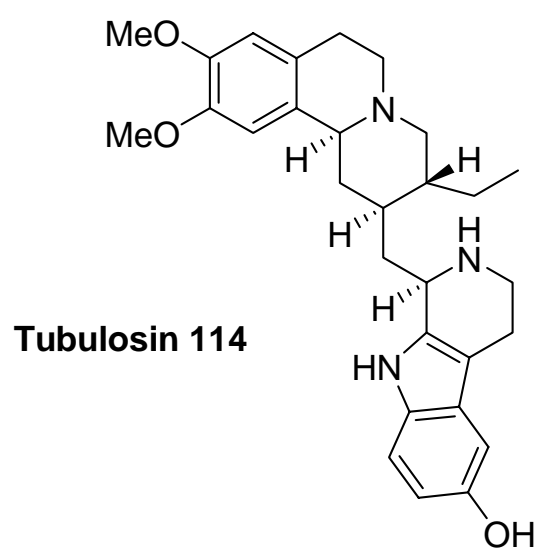

Abbildung 33: Alkaloide, die durch eine Domino Knoevenagel-hetero-Diels-Alder-Reaktions nach Tietze aufgebaut worden sind. 
Hirtusin 111, ein Indol-Alkaloid der Corynanthe-Klasse, wurde aus der Pflanze Uncaria rhynchophylla isoliert und für die Herstellung der chinesischen Volksmedizin „Kampo“ eingesetzt. Seine pharmakologische Wirkung besteht in einer erheblichen Inhibition des Influenza A Virus (Untergruppe H3N2), welche mit einem $E C_{50}=0.40-0.57 \mu \mathrm{g} / \mathrm{ml}, 11-20$-mal höher als die des klinisch angewendeten Ribavirin ist. Die retrosynthetische Analyse von Hirsutin 111 führt zum (3R)-Tetrahydro- $\beta$ carbolinacetaldehyd 118. Meldrum-Säure 108 und dem Enolether 119 über die Zwischenstufen 115-117 (Abbildung 34).

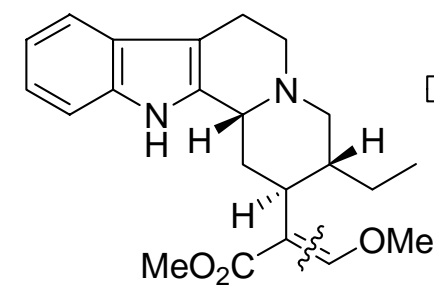

Hirsutin 111
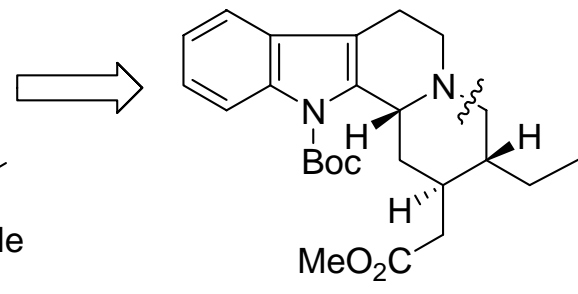

115
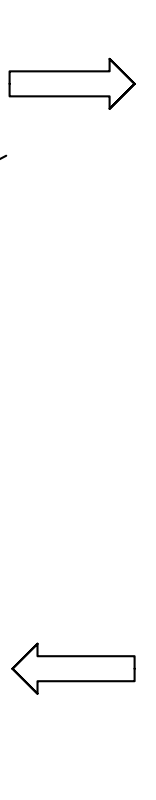<smiles>CCC(C=O)C(C)C[C@H]1c2c(c3ccccc3n2C(=O)c2ccccc2)CCN1C(=O)c1ccccc1</smiles>

116

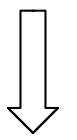

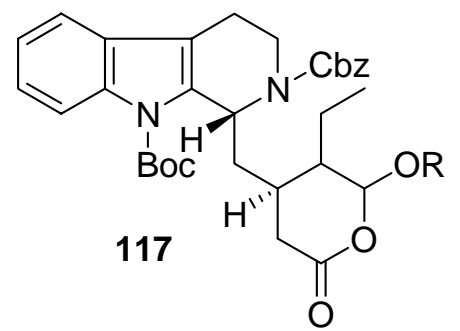

Abbildung 34: Retrosynthese Analyse von Hirsutin (111).

\subsection{Syntheseplanung}

In Anlehnung an die Arbeiten von J. Wichmann, U. Griesbach, N. Rackelmann und Y. Zhou ergab sich für die vorliegende Arbeit die Augabe, mit Hilfe einer DreiKomponenten-Domino-Knoevenagel-hetero-Diels-Alder-Reaktion, gefolgt von einer weiteren Domino-Sequenz, bestehend aus Solvolyse, Hydrogenolyse, Kondensation 
und Hydrierung das Yohimban-Alkaloid Yohimbin (9) und das Camptotheca-Alkaloid Camptothecin (44) enantiomerenrein aufzubauen.

Die retrosynthetische Analyse von Yohimbin (9) erfolgte unter der Option, den Domino-Knoevenagel-hetero-Diels-Alder-Prozess als Schlüsselreaktion einzusetzen. Das Lacton 120 könnte man so auf den enantiomerenreinen Aldehyd (1S)-121 sowie auf den Enolether 122, der als Dienophil dient, und die käufliche Meldrum-Säure (108) zurückführen (Abbildung 35).<smiles>CO[C@@H]1[C@H]2C[C@@H]3c4[nH]c5ccccc5c4CCN3C[C@]2(C)CC[C@H]1O</smiles>

imbin 9

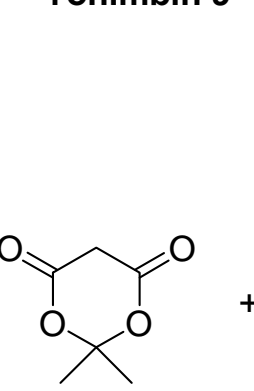

108

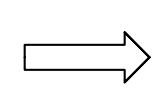

120

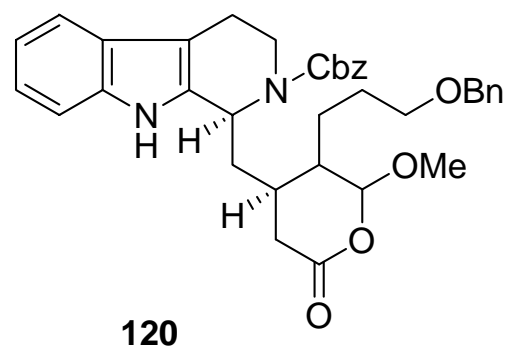
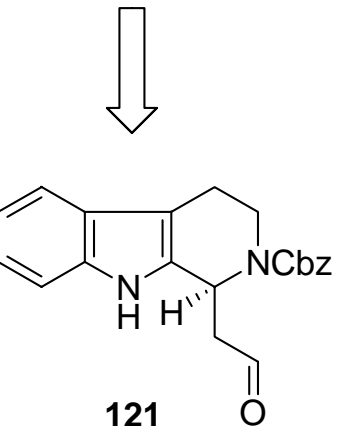

Abbildung 35: Retrosynthetische Analyse von Yohimbin (9).

Daraus ergab sich als erstes Ziel, den enantiomerenreinen Aldehyd (1S)-121 aus dem racemischen Indol-Derivat 123 darzustellen (Abbildung 36). Die Reaktionssequenz sollte mit dem kommerziell erhältlichen Tryptamin Hydrochlorid (17) beginnen, der zum Imin 124 umgewandelt würde. Für die selektive Einführung des stereogenen Zentrums des chiralen Carbolins 121 sollte eine von Noyori entwickelte enantioselektive Hydrierung einer $\mathrm{C}=\mathrm{N}$-Doppelbindung dienen. Hierzu sollte die racemische Verbindung 123 zuvor zum Imin 124 oxidiert und nachfolgend mit Hilfe einer asymmetrischen Ruthenium-katalysierten Transferhydrierung mit $\mathrm{HCO}_{2} \mathrm{H} / \mathrm{NEt}_{3}$ in 
Gegenwart des Katalysators $(R, R)-\mathbf{1 2 5}$ zum enantioselektiven Indol-Aldehyd (1S)-121 umgesetzt werden.<smiles>CCOC(=O)CC(=O)OCCNCCc1c[nH]c2ccccc12</smiles>

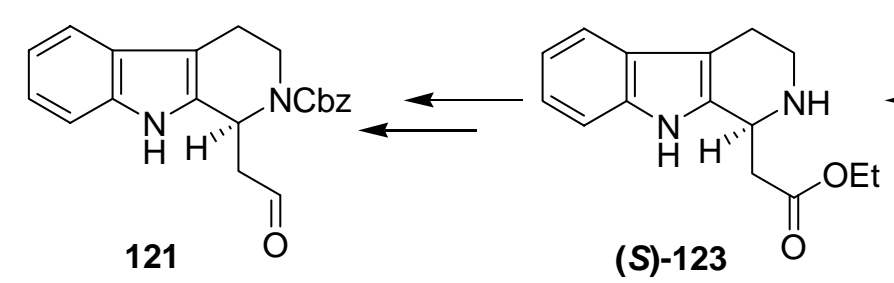<smiles>[R16]C(C)[R16]([H])([H])CC</smiles><smiles>CCOC(=O)CC1NCCc2c1[nH]c1ccccc21</smiles>

125

Abbildung 36: Synthese des enantiomerenreinen Aldehyds (1S)-121.

Zur Synthese des erforderlichen Enolethers 122 sollte 1,5-Pentandiol (127) in mehreren Transformationen $\mathrm{zu}$ einem Acetal umgesetzt und aus diesem in einer Eliminierungsreaktion die ungesätitgte Verbindung 122 gebildet werden (Abbildung $37)$.<smiles>OCCCCCO</smiles>

127<smiles>COC=CCCCOCc1ccccc1</smiles>

122

Abbildung 37: Synthese des Enolethers 122.

Der Aldehyd 121 und der Enolether 122 sollten zusammen mit der käuflichen Meldrum-Säure (108) anschließend in einer Domino-Knoevenagel-hetero-Diels-AlderReaktion zu den entsprechenden Lactonen 120a und 120b umgesetzt werden. (Abbildung 38). Die Beeinflussung der facialen Selektivität durch die Einführung 
eines Substituenten R am Indol-Stickstoff wurde schon bei früheren Untersuchungen im Arbeitskreis Tietze beobachtet und sollte auch hier zur Selektivitätssteigerung des Domino-Prozesses gegebenenfalls genutzt werden.

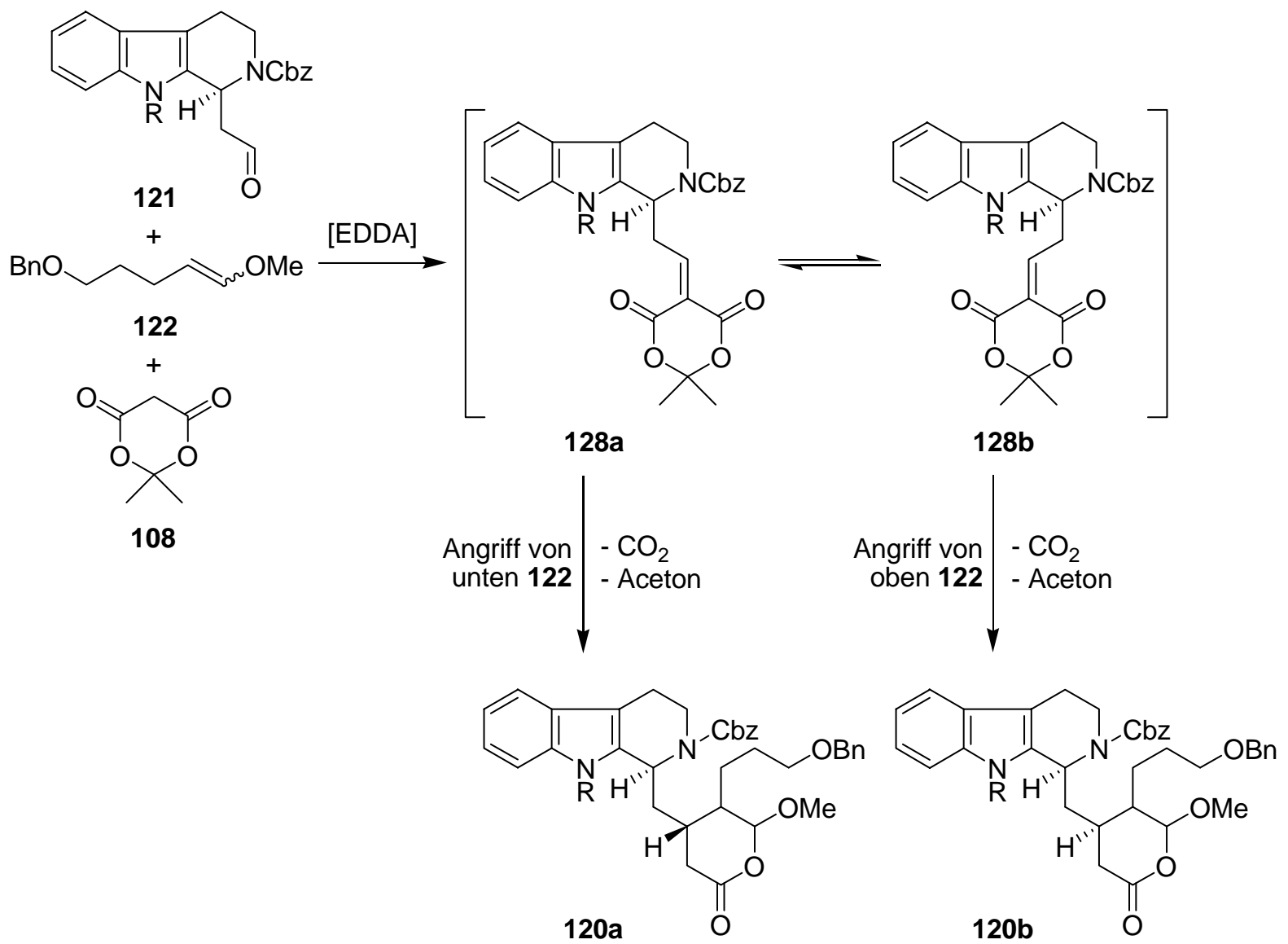

Abbildung 38: Einfluss von Substituenten am Indol-Stickstoff auf die Lage des Konformerengleichgewichts.

Eine weiterere Aufgabe, die im Rahmen dieser Arbeit untersucht werden sollte, war das an Yohimbin (9) vorgestellte Synthesekonzept auf die Totalsynthese von Camptothecin (44) zu erweitern. Die erfolgreiche Anwendung der DominoKnoevenagel-hetero-Diels-Alder-Reaktion würde so einen völlig neuen synthetischen Zugang zum Aufbau von Camptotheca-Alkaloiden erlauben.

Der nachfolgend vorgestellte retrosynthestische Ansatz ist an dem von Yohimbin (9) angelehnt. Ein effizienter Aufbau des Zielmoleküls sollte über eine Domino-Knoevenagel-hetero-Diels-Alder-Umsetzung erfolgen, gefolgt von einer biomimetischen 
Cyclisierung. Substrate für diese Reaktion sind die kommerziell erhältliche MeldrumSäure (108), der als Dienophil fungierende Silylenolether 129 und der enantiomerenreine Aldehyd 130 (Abbildung 39). Letzteres würde enantioselektiv durch eine vorangegangene asymmetrische Hydrierung eines zuvor gebildeten Imins nach Noyori aufgebaut werden.<smiles>CCC1(O)C(=O)OCc2c1cc1n(c2=O)Cc2cc3ccccc3nc2-1</smiles>

Camptothecin 44

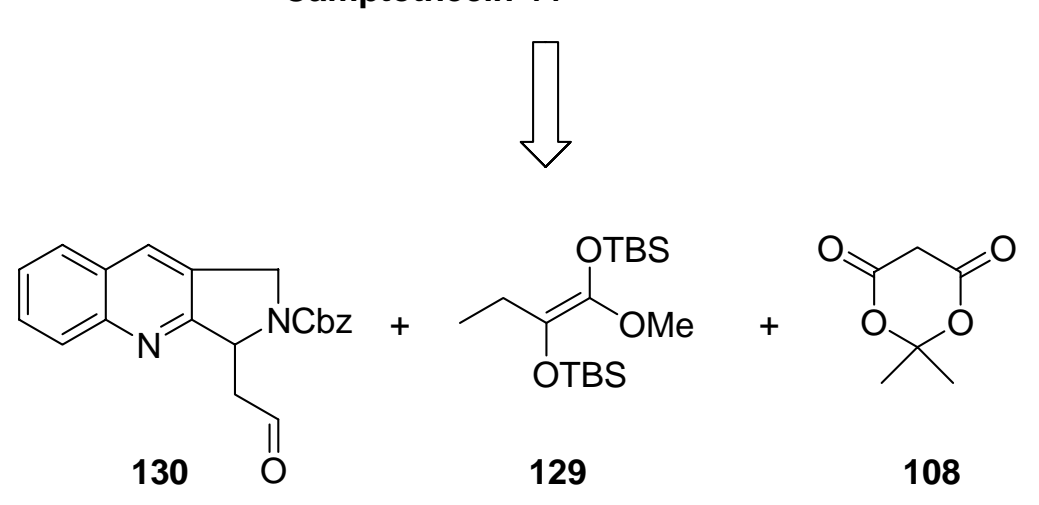

Abbildung 39: Retrosyntheseschema zum Aufbau von Camptothecin (44).

Zur Synthese des Silylenolethers 129 bietet sich an, von der Ketocarbonsäure 131 auszugehen. Eine fünfstufige Synthesesequenz sollte es erlauben, das Dienophil 129 erfolgreich aufzubauen (Abbildung 40).<smiles>CCC(=O)C(=O)O</smiles>

131<smiles>CC/C([OH2+])=C(/OC)O[SbH3]</smiles>

129

Abbildung 40: Synthese des Dienophils 129. 
Zusammenfassend ergaben sich also folgende Aufgaben:

A) Enantioselektive Totalsynthese von Yohimbin

- Synthese des ennantiomerenreinen Aldehyds (1S)-121

- Synthese des Enolethers 122

- Synthese des Indol-Alkaloids Yohimbin (4) über eine Domino-Knoevenagelhetero-Diels-Alder-Sequenz als Schlüsselschritt

- Untersuchungen zur Faciale Differenzierung der Domino-Knoevenagel-heteroDiels-Alder-Reaktion durch Variation des Substituenten am Indol-Stickstoff

B) Enantioselektive Totalsynthese von Camptothecin

- Synthese des ennantiomerenreinen Aldehyds (1S)-130

- Synthese des Silylenolethers 129

- Synthese des Chinolin-Alkaloids Camptothecin (44) über eine DominoKnoevenagel-hetero-Diels-Alder-Sequenz als Schlüsselschritt 


\section{A) Versuche zur Synthese von Yohimin (9)}

\section{Synthese des Enolethers 122}

\subsection{Versuche zur Synthese des Enolethers 122}

Die Darstellung einfacher Enolether wurde in der Literatur von J. Bachmann über eine säurekatalysierte Eliminierung eines Alkohols aus den entsprechenden Aldehydacetalen beschrieben. Diese gebräuchliche Methode sollte auch im vorliegenden Fall zum Einsatz kommen. Ausgehend vom kommerziell erhältlichen 1,5-Pentandiol (127) wurde eine Hydroxylgruppe mit TBSCl in Gegenwart von Imidazol in DMF in einer sehr guten Ausbeute von $92 \%$ geschützt. Der monogeschützte Alkohol 132 wurde anschließend durch eine Oxidation mit $\mathrm{NaClO}$ und katalytischen Mengen an TEMPO in den Aldehyd 133 überführt und dieser wiederum im letzten Schritt nach einer literaturbekannten Vorschrift in das gewünschte Acetal 134 umgewandelt (Abbildung 41).

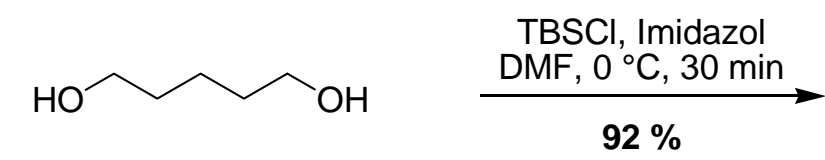

127

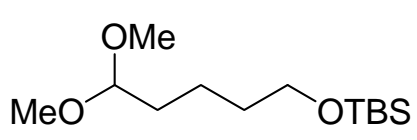

134

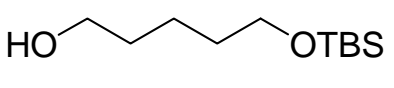

132

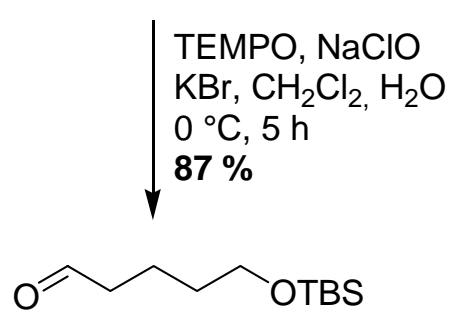

133

Abbildung 41: Synthese des Acetals 134.

Trotz wiederholter Versuche gelang es jedoch nicht die Synthese von 135 über einen $\mathrm{KHSO}_{4}$ vermittelten Eliminierungsprozess durchzuführen, obwohl in früheren 
Arbeiten Enolether wie 1-(4-Methoxy)-buten und 1-Isopropoxylbuten in guten Ausbeuten über diesen Reaktionsweg zugänglich waren (Abbildung 42).

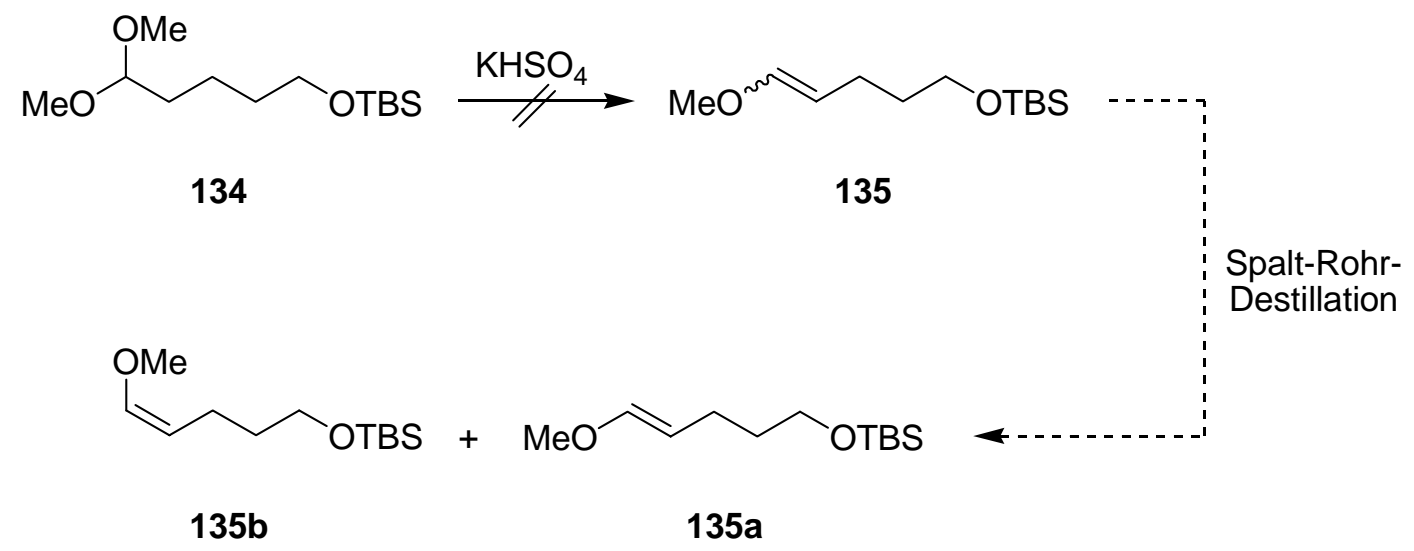

Abbildung 42: Versuche zur Synthese des Enolethers 135 mit einer Säure-katalysierten Eliminierung.

Die eigentliche Synthesevorschrift sah vor, zu dem auf ungefähr $80^{\circ} \mathrm{C}$ erwärmten Acetal 134 eine katalytische Menge von Kaliumhydrogensulfat zuzugeben und das dabei entstehende Produkt über eine Vigreux-Kolonne in eine mit wenig Kaliumcarbonat beschickte Vorlage zu destillieren. Da sich das Acetal 134 jedoch als eine bei $80{ }^{\circ} \mathrm{C}$ siedende und dadurch leicht flüchtige Verbindung herausstellte, wurde erstmals die Säure bei Raumtemperatur zugegeben. Das dabei gewonnene Destillat konnte dann aber nur als Ausgangsverbindung identifiziert werden. Daraufhin wurde $\mathrm{KHSO}_{4}$ bei unterschiedlichen Temperaturen zwischen 50 und $75{ }^{\circ} \mathrm{C}$ zugegeben, wobei keine Umsetzung zu 135 erreicht werden konnte. Stattdessen verwandelte sich das farblose und flüssige Substrat in eine schwarze, klebrige Masse, was darauf schließen lässt, dass eine Polymerisation des Eduktes stattgefunden haben muss. Um dennoch eine Umsetzung zu bewirken, wurde versucht, durch das Einleiten eines Stickstoffstroms das als eines der beiden Eliminierungsprodukte eventuell gebildete Methanol auf diese Art und Weise aus dem Reaktionsgleichgewicht zu entfernen und somit eine Verschiebung des Prozesses in Richtung des Enolethers zu ermöglichen. Dieser Versuch blieb jedoch erfolglos. Weitere Versuche, durch Variation der Säure den 
Enolether 135 zugänglich zu machen, blieben ebenfalls ergebnislos. Weder $p$ Toluolsulfonsäure noch Diphenylphosphinsäure führten zum Erfolg.

\subsection{Synthese des Enolethers 122}

Die missglückten Versuche zur Synthese von Verbindung 135 legten die Überlegung nahe, anstelle von 134 ein Acetal mit veränderter Alkoholschutzgruppe der Eliminierungsreaktion zum entsprechenden Enolether auszusetzen. Die Wahl fiel auf das benzylgeschützte Derivat 122 (Abbildung 43).

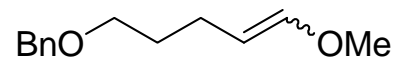

122

Abbildung 43: Alternativer Enolether 122 für die Domino-Knoevenagel-hetero-Diels-Alder-Reaktion.

Eine Möglichkeit, Substrat 122 darzustellen, basierte auf der einfachen Schützung von 1,5-Pentandiol (127) mit Benzylbromid. Der gewünschte Benzylether (136) konnte so in sehr guter Ausbeute von $88 \%$ erhalten werden (Abbildung 44).

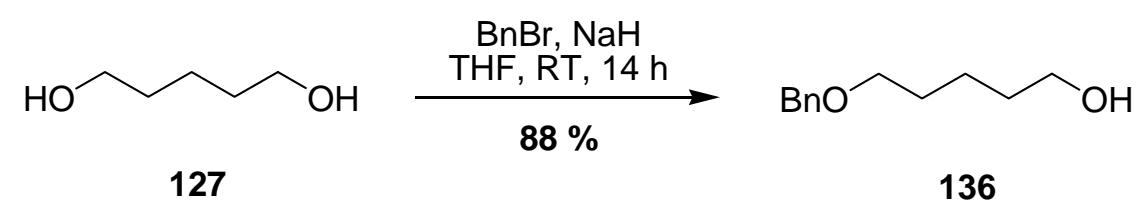

Abbildung 44: Synthese des monogeschützten Diols 136.

In einer alternativen Synthesestrategie wurde das zuvor bereitgestellte und nun nicht mehr gebräuchliche Molekül 132 als Ausgangsverbindung verwendet. Analog der Benzylierung von 1,5-Pentandiol (127) ließ sich hier eine Benzyl-Schutzgruppe einführen. Zu diesem Zweck wurde der Ether 132 in THF gelöst und mit Benzylbromid umgesetzt, wobei $60 \%$-iges Natriumhydrid (in Weißöl) als Base diente. Im Anschluss daran wurde die TBS-Schutzgruppe mit Hilfe von Tetrabutylammonium- 
fluorid entfernt. Es folgten die bereits aus der Darstellung von 134 bekannten Transformationen, die Oxidation der Hydroxylgruppe zum Aldehyd und die Acetalbildung. Die Bildung des Enolethers im letzten Schritt der Synthese des Dienophils gestalltete sich jedoch auch mit verändeter Schutzgruppe als problematisch. Zwar handelt es sich bei 139 um keine leicht flüchtige Verbindung, dennoch ließ sich das Acetal 139 wie sein Vorgänger 134 nicht zu einer Eliminierungsreaktion veranlassen (Abbildung 45).

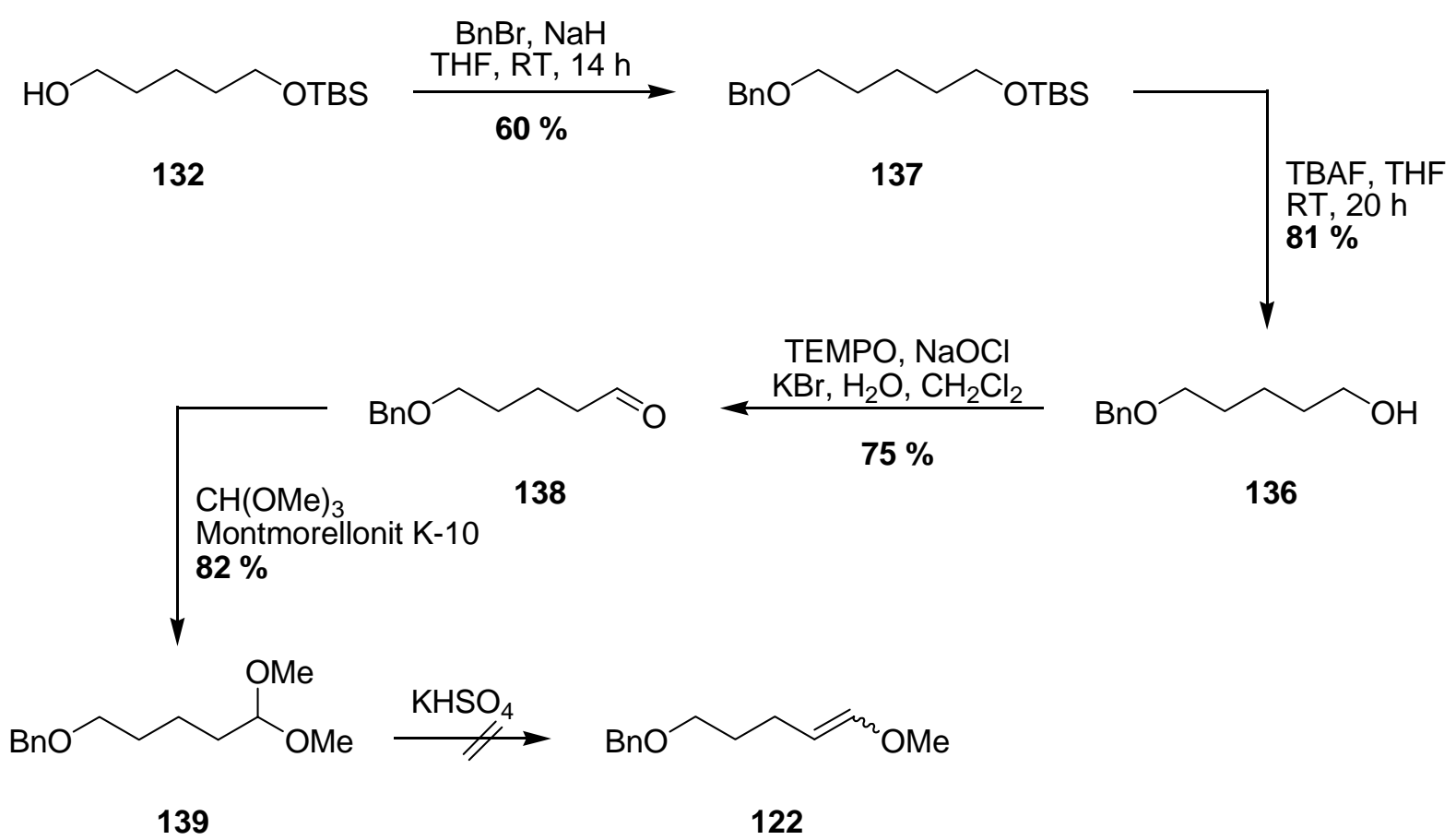

Abbildung 45: Versuche zur Synthese des Enolethers 122 über eine Eliminierungreaktion.

Grundsätzlich ist die einstufige Synthese von 136 (Abbildung 44) der zweistufigen (Abbildung 45) vorzuziehen und insbesondere aus Sicht von ökologischen und ökonomischen Aspekten sinnvoll. Zusätzlich gewinnt der erste Weg an Attraktivität beim Vergleich der Ausbeuten, konnte Verbindung 136 doch in $88 \%$ Ausbeute erhalten werden, während der zweite Weg nur eine Gesamtausbeute von 65 \% liefert.

Die erfolglosen Bemühungen, die Enolether 135 und 122 über eine säurekatalysierte Eliminierung von einem Alkohol aus einem Aldehydacetal zugänglich zu machen, 
machten eine neue Synthesestrategie unumgänglich. In einem alternativen Konzept wurde daher versucht, über eine Wittig-Reaktion zu einem Enolether vom Typ 122 zu gelangen. Dafür wurde die Synthese von 4-Hydroxybutanal (142) angestrebt, welches sich im Gleichgewicht $\mathrm{zu}$ seiner cyclischen Lactol-Form (141) befindet. Am sinnvollsten erschien es, den Aldehyd 142 über eine säurekatalysierte Addition von Wasser an die Doppelbindung von 2,3-Dihydrofuran zu synthetisieren. Dieser Ansatz stellte sich jedoch nur als bedingt durchführbar heraus. Die Umsetzung zur gewünschten Verbindung 141 ließ sich zwar realisieren, doch wurde dieser Erfolg durch den Umstand, dass es zur Ausbildung eines Trimers (143) kam, stark beeinträchtigt (Abbildung 46). Da Verbindung 143 als Hauptprodukt in einem Verhältnis von 2.7:1.0 zum Lactol 141 vorlag, lag der Anteil an 141 bezüglich der Geamtausbeute des Gemisches bei nicht zufriedend stellenden $19 \%$.

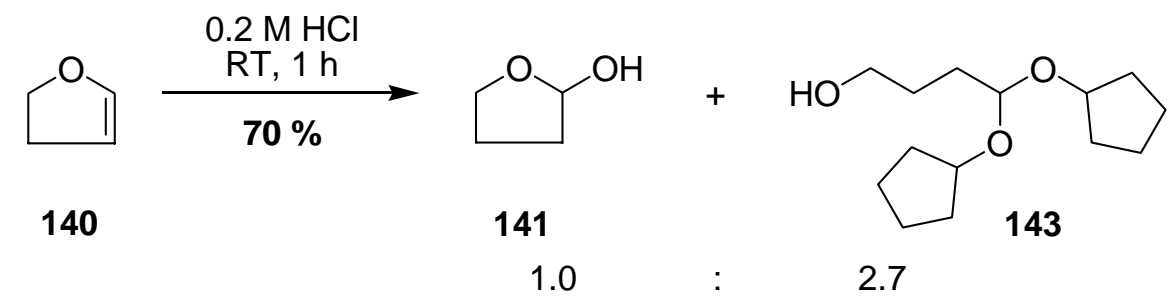

Abbildung 46: Versuch zur Synthese des Lactols 141 mittels einer Additionsreaktion.

Die Suche nach Ursachen legte die Überlegung nahe, dass durch den Einsatz einer schwächeren Säure als Katalysator die Bildung des Trimers 143 unterbunden werden könnte. Die Wahl fiel auf para-Toluolsulfonsäure, deren Acidität geringer als die von Salzsäure ist. Mit dem Einsatz eines 2-Phasen-Lösungsmittelsystems bestehend aus Diethylether und Wasser sollte dann das entstehende Lactol möglichst schnell aus der wässrigen Phase in die organische überführt werden, um Verluste möglichst gering halten (Abbildung 47). Leider konnte auch nach einer Reaktionszeit von $14 \mathrm{~h}$ dünnschichtchromatographisch kein Umsatz beobachtet werden, so dass dieser Ansatz nicht weiter verfolgt wurde. 


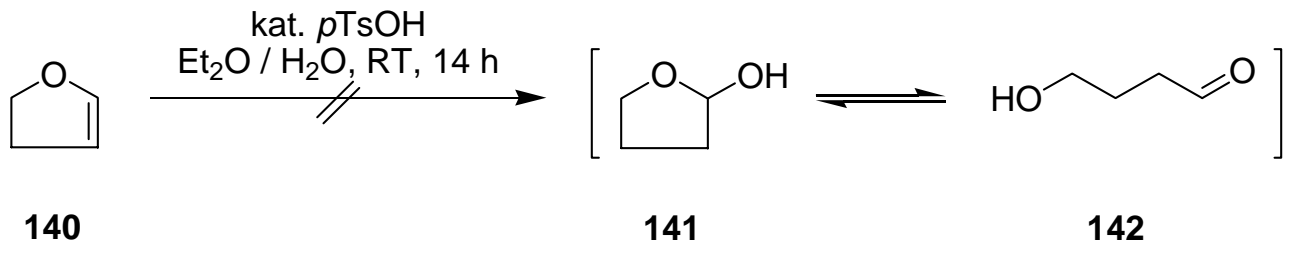

Abbildung 47: Versuche zur Synthese des Lactols 141.

Eine weitere Möglichkeit zur Darstellung von 141 bot die Reduktion der KetoFunktionalität von $\gamma$-Butyrolacton (144) an. Der Einsatz von Diisobutylaluminiumhydrid als Reduktionsmittel erlaubte eine vollständige Umsetzung des Lactons 144 zum gewünschten Aldehyd 142, das sich wie bereits erwähnt im Gleichgewicht zu seinem Lactol 141 befindet.

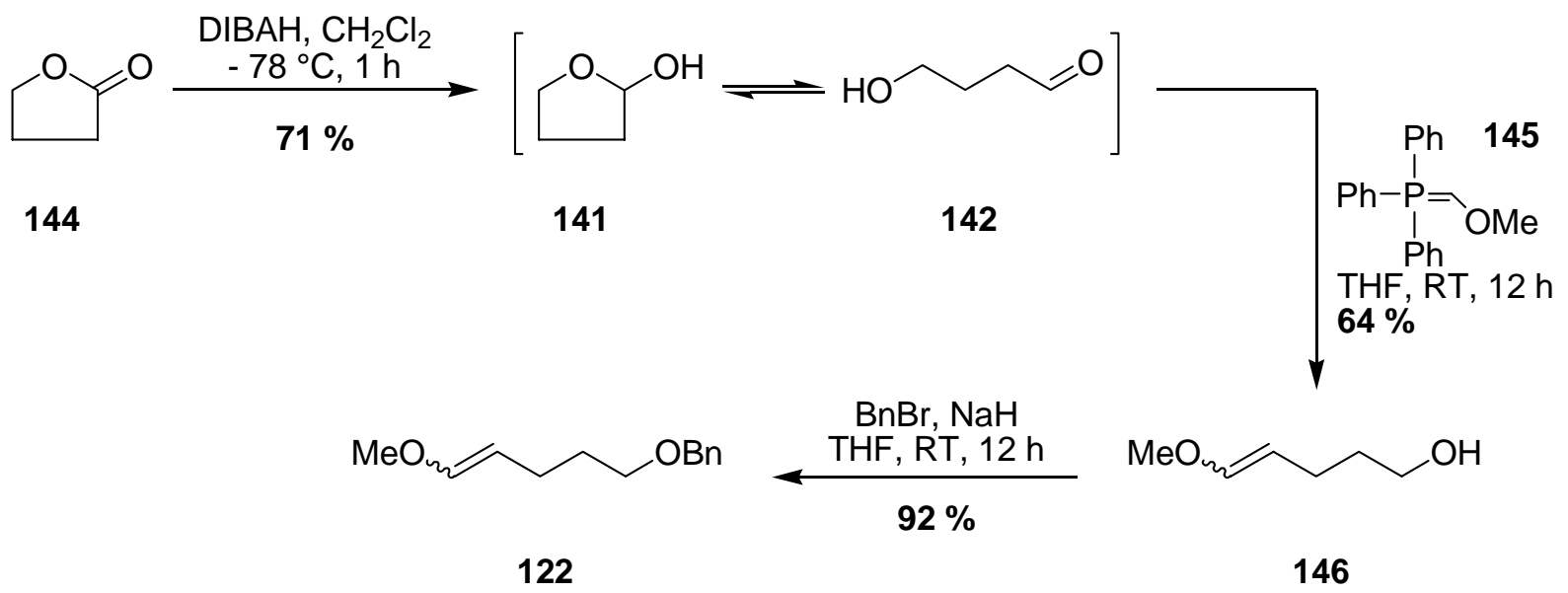

Abbildung 48: $\quad$ Synthese des Enolethers 122.

Die Isolierung der gesamten Menge an der kleinen und polaren Verbindung 142 aus der wässrigen Phase konnte jedoch nicht erreicht werden. Reaktionen, die über einen Ansatz von $5 \mathrm{~g} \gamma$-Butyrolacton (144) hinausgingen, resultierten in einem erheblichen Verlust der Ausbeute an 141 (30-50 \%). Die besten Ergebnisse wurden mit Ansätzen zwischen $1 \mathrm{~g}$ und $2.5 \mathrm{~g}$ erreicht. Im Anschluss an die Reduktion sollte dann eine Wittig-Reaktion erfolgen. Für die Durchführbarkeit dieser Transformation musste im ersten Schritt aus (Methoxymethyl)triphenylphosphoniumchlorid in situ das Phosphor- 
Ylen 145 generiert werden, indem tropfenweise zu seiner Suspension in THF bei $-78^{\circ} \mathrm{C}$ langsam eine $n$-Buthyllithium-Lösung zugegeben wurde. Die Entstehung des Ylens 145 zeigte sich dabei in der charakteristisch dunkelroten Verfärbung der Suspension, die zur Vervollständigung der Reaktion eine Stunde bei $-78{ }^{\circ} \mathrm{C}$ gerührt wurde. Alternativ konnte auch s-Butyllithium als Base eingesetzt werden. Das Zufügen von 4-Hydroxybutanal (141) erlaubte nach $14 \mathrm{~h}$ die Umsetzung zum entsprechenden Enolether 146 in $64 \%$ Ausbeute. Letztendlich vollendete die Schützung der freien Alkoholgruppe von 146 mit Benzylbromid die erfolgreiche Synthese des für den Domino-Knoevenagel-hetero-Diels-Alder-Prozesses notwenigen Dienophils 122 (Abbildung 48).

\subsection{Versuche zur Synthese weiterer Enolether}

Anstelle eines benzylgeschützten Enolethers (122) wurden auch Versuche unternommen, Derivate dieser Verbindung herzustellen, um einerseits die Produktpalette der Indolalkaloide vom Yohimban-Typ zu erweitern und andererseits, um die Leistungsfähigkeit der Domino-Knoevenagel-hetero-Diels-Alder-Reaktion zu überprüfen. Aus einer schier unbegrenzten Anzahl an unterschiedlichsten Substraten, die als Dienophil für den Domino-Prozess als geeignet erschienen, wurde das geschützte Diol 157 ausgesucht. Diese Wahl lässt sich darauf begründen, dass die Entschützung des Diols den Zugang zu vielfältigen, an der Seitenkette in C-20 Position funktionalisierten Indolalkaloiden gestattet. Von den unterschiedlichen Synthesestrategien, die einen Zugang zum Triol 147 erlauben, wurden zwei Transformationen untersucht (Abbildung 49). Zum Einen wurde Pent-4-en-1-ol (148) an der terminalen Doppelbindung dihydroxyliert. Die üblicherweise verwendeten Reagenzien sind katalytische Mengen an Osmium(IV)-tetraoxid und der stöchimetrischer Einsatz von $N$-Methylmorpholin- $N$-oxid, wobei alternativ in diesem Fall Trimethylamin- $N$-oxid als Oxidationsmittel verwendet wurde. Schwierigkeiten bereitete bei dieser Reaktion die Anwesenheit von Wasser als ein Lösungsmittelbestandteil neben tert-Butanol. Aufgrund der hohen Polarität des Triols 147 gelang die Überführung aus der wässrigen 
in die organische Phase nur unzureichend mit einer Aubeute von nur moderaten $12 \%$. Deswegen erschien In diesem Fall der zweite Weg, die Reduktion von 2Oxopentandicarbonsäure zu 147 als sinnvoller. Mit Hilfe von Lithiumaluminiumhydrid konnte die Dicarbonsäure 149 jedoch nur unzureichend zum Triol 147 reduziert werden. Die Hydrolyse des noch verbliebenen Lithiumaluminium-hydrids sollte mit einer geringen Menge von Wasser erfolgen. Überschüssiges Wasser sollte anschließend durch Zugabe von $\mathrm{MgSO}_{4}$ gebunden werden, wobei eine gräulich-weiße Suspension entstehen sollte. Stattdessen beobachtete man ein Verklumpen des Reaktionsgemisches, so dass sich das Abtrennen von $\mathrm{LiOH}$ und $\mathrm{MgSO}_{4}$ als sehr problematisch erwies. Obwohl der Filterkuchen mit reichlich Lösungsmittel gespült wurde, konnte nicht mehr als $18 \%$ des Produktes 147 gewonnen werden. Möglicherweise lässt sich dieser Befund auf die Alterung des sehr empfindlichen Reagenzes zurückzuführen. Dennoch reichten die geringen isolierten Mengen an 147 aus, um nachfolgend die Alkohlfunktinalitäten in Position C-1 und C-2 mit 2,2Dimethoxypropan zu schützen (Abbildung 49).

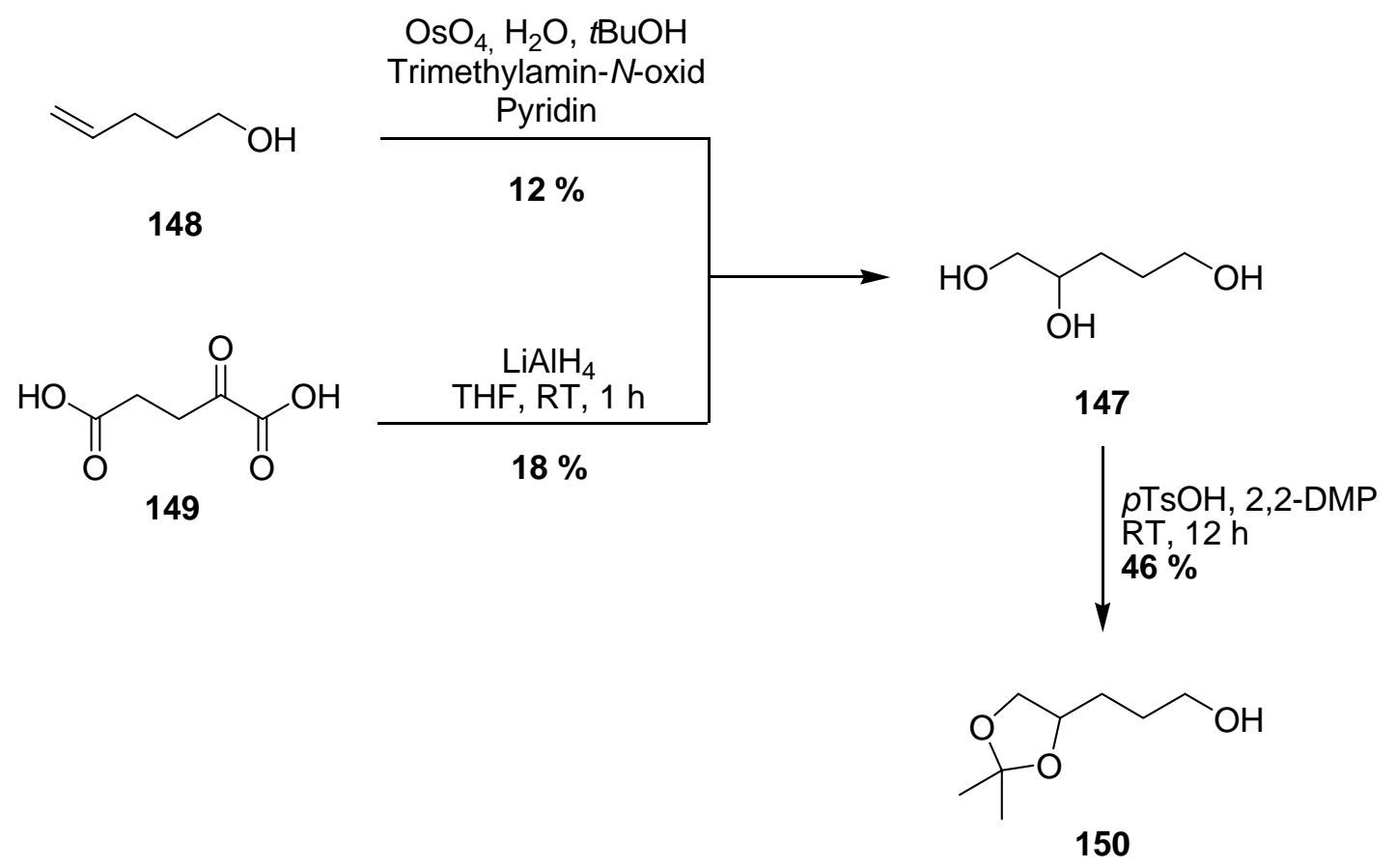

Abbildung 49: Synthese des Alkohols 150 über zwei Synthesestrategien. 
Die problematische Darstellung von dem Triol 147 über die beschriebenen Umsetzungen (Abbildung 49) erforderte eine neue Strategie. Als interessante Alternative bot sich die von C. L. Wilson beschriebene Reaktion von Tetrahydrofuran- und Tetrahydropyran-Substraten mit Reagenzien wie Acetanhydrid oder Wasserstoffbromid zu offenkettigen Butan- oder Pentan-Derivaten an. Ziel war es, 1,2,5-Triacetoxypentan (151) ausgehend vom käuflichen Tetrahydrofurfurylalkohol (152) über eine Reaktion mit Zinkchlorid und Acetanhydrid zu erhalten. Dazu wurde das Edukt 152 solange mit Acetanhydrid als Lösungsmittel refluxiert, bis es dünnschichtchromatographisch nicht mehr detektierbar war. Fraktionierte Destillation der schwarz gewordenen Reaktionslösung ergab zwei Produkte, zum einen das gewünschte 1,2,5-Triacetoxypentan (151) und zum anderen Monoacetoxypentan 153. Die Gesamtausbeute beider Verbindungen belief sich dabei auf $81 \%$ (Abbildung 50).

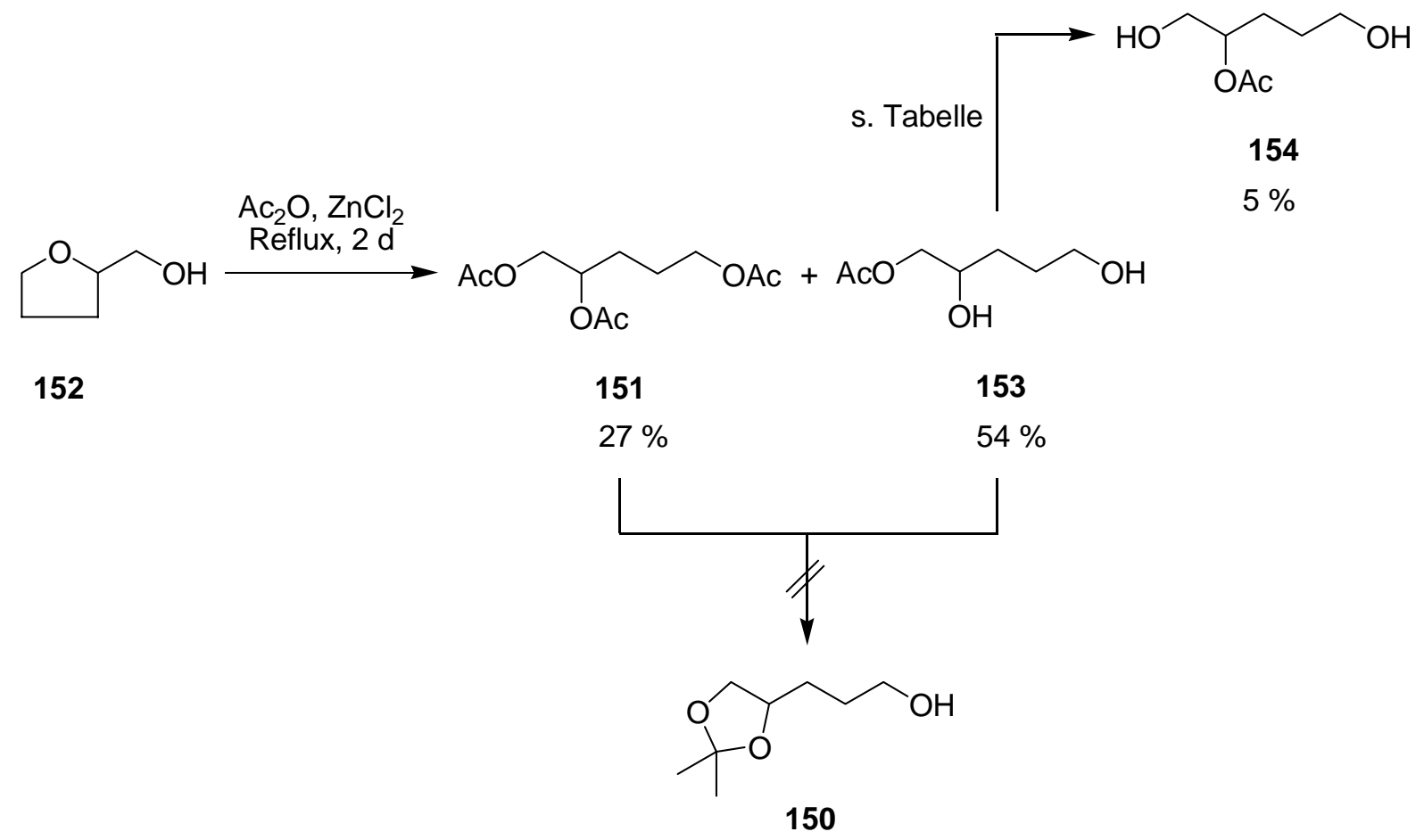

Abbildung 50: Versuche zur Synthese der Triacetoxy-Verbindung 151.

Bei dem einfach acylierten Molekül 153 ließ sich aus den spektroskopischen Daten zunächst nicht erkennen, an welcher Position sich die Acetoxy-Gruppe befindet. Daher 
wurde versucht, 153 analog dem Triol 151 mit 2,2-Dimethoxypropan zu schützen. Hätte sich die Acetoxy-Funktionalität in Position C-5 befunden, sollte eine Umsetzung beobachtet werden müssen. Da dies aber nicht der Fall war, kann man zumindest mit Sicherheit ausschließen, dass es sich um 5-Acetoxy-1,2-pentandiol handelt. Eine eindeutige Aussage darüber, ob es sich nun um Verbindung 153 oder das Regioisomer 2-Acetoxy-1,5-pentandiol handelt, konnte nicht getroffen werden. Einfachhalber wurde in Abbildung 50 auf die Darstellung des zweiten in Frage kommenden Isomers verzichtet.

Wenngleich vorangegangene Versuche, die Monoacetoxy-Verbindung 153 mit 2,2Dimethoxypropan zu schützen, missglückt waren, wurde angenommen, die an sich unter leicht sauren Reaktionsbedingungen abspaltbare Acetoxy-Funktionalität sowohl bei 151 als auch bei 153 erfolgreich entfernen zu können. Hierzu wurde versucht, unter Verwendung unterschiedlicher saurer Reagenzien, den Alkohol 147 zu erhalten. Während die Spaltung der Acetyl-Gruppe beim Diol 153 im geringen Maße zu einer Acylgruppenwanderung führte (Tabelle 2), blieb 1,2,5-Triacetoxypentan (161) vollständig unangetastet. Weitere Untersuchungen zu diesem Syntheseansatz wurden deswegen eingestellt.

\begin{tabular}{cccc}
\hline Nr. & Substrat & Reaktionsbedingungen & Ergebnis \\
\hline 1 & $\mathbf{1 5 3}$ & IR-120, Aceton, RT, 2 d & Acylgruppen-Wanderung \\
2 & $\mathbf{1 5 3}$ & $p$ TsOH, Aceton, RT, 2 d & Acylgruppen-Wanderung \\
3 & $\mathbf{1 5 3}$ & $p$ TsOH, 2,2-DMP, RT, 2 d & Acylgruppen-Wanderung \\
4 & $\mathbf{1 5 1}$ & IR-120, Aceton, RT, 2 d & keine Umsetzung \\
5 & $\mathbf{1 5 1}$ & $p$ TsOH, Aceton, RT, 2 d & keine Umsetzung \\
6 & $\mathbf{1 5 1}$ & $p$ TsOH, 2,2-DMP, RT, 2 d & keine Umsetzung \\
\hline
\end{tabular}

Tabelle 2: Versuche zur Abstaltung der Acetoxy-Funktionalität bei 153 und 151.

Darüberhinaus sah die eigentliche Syntheseplanung vor, den Alkohol 150 in drei aufeinanderfolgenden Schritten, bestehend aus einer Oxidation, einer Acetalbildung 
und einer Eliminierung, zum Enolether 157 umzusetzen. Dabei war es, aufgrund der an anderen Substraten bereits festgestellten Problemen anzunehmen, die Säurekatalysierte Eliminierungsreaktion, in diesem Fall nicht zum gewünschten Dienophil 157 geführt hätte.

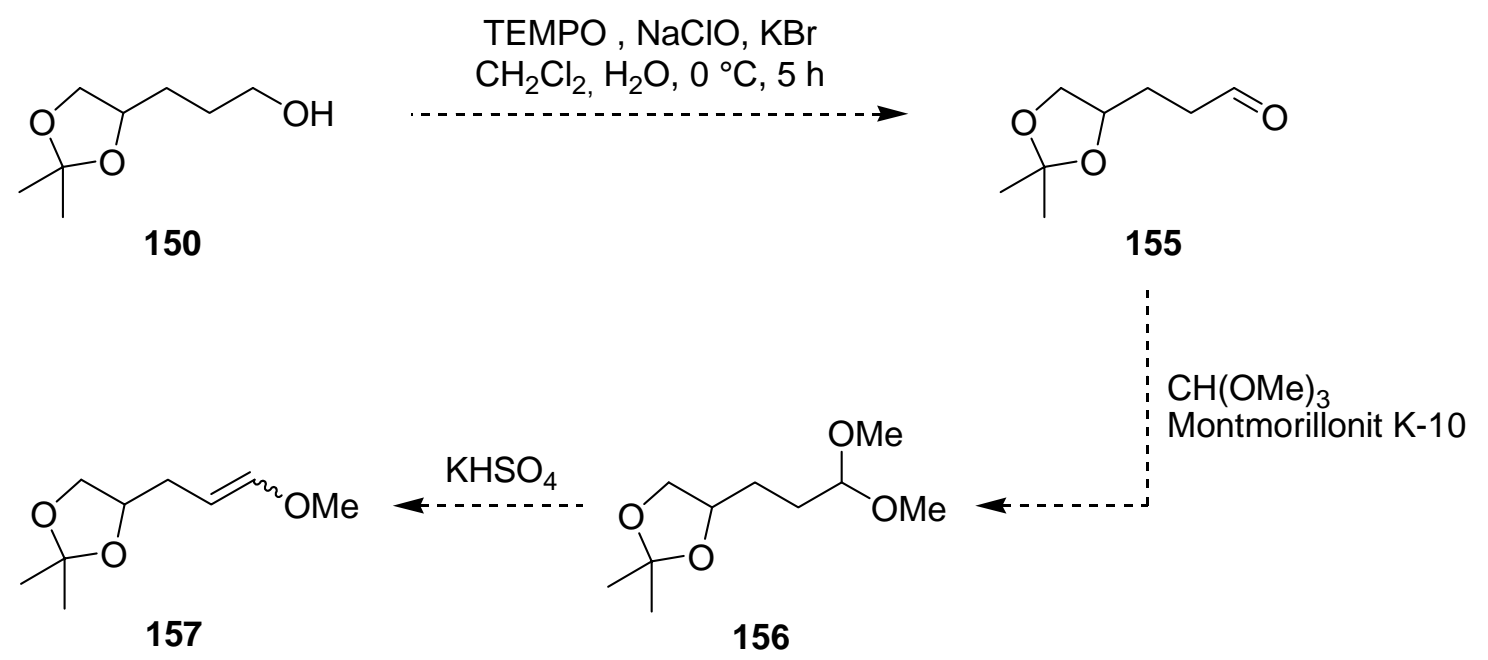

Abbildung 51: Synthesestrategie für die Darstellung von dem Enolether 157. 


\section{Synthese des Aldehyds 121}

\subsection{Versuche zur Synthese des enantiomerenreinen (1S)-Tetrahydroiso- chinolins 121}

Ein wesentlicher Aspekt der Synthese von Yohimbin (9) und eventueller Analoga war die Bildung des enantiomerenreinen Aldehyds (1S)-121, der in der DominoKnoevenagel-hetero-Diels-Alder-Reaktion eingesetzt werden sollte. Das stereogene Zentrum C-1 sollte über einen katalytischen Prozess eingeführt werden. Zuvor war aber als ein Baustein des Aldehyds 121 die $\alpha$-Ketocarbonsäure 126 zu synthetisieren. Einen einfachen und vor allem auch kostengünstigen Zugang dazu stellte Diethylnatriumoxalacetat (158) dar. Dieses wurde mittels einer Verseifung und anschließender saurer Aufarbeitung in die entsprechende Säure 126 überführt (Abbildung 52).<smiles>CCOC(=O)/C=C(\O[N+]([O-])OCC)C(=O)OCC</smiles>

158

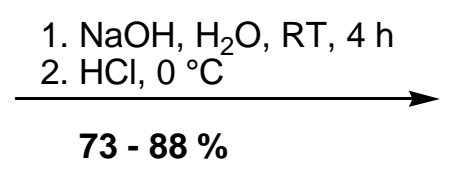

$73-88 \%$<smiles>CCOC(=O)CC(=O)C(=O)O</smiles>

126

Abbildung 52: Synthese des Ketoesters 126 für die Pictet-Sprengler-Reaktion.

Als effizienteste Methode zur Synthese von 1,2,3,4-Tetrahydro- $\beta$-carbolin-Systemen vom Typ 123 zeichnet sich die Pictet-Sprengler-Reaktion aus. Auch in der Biosynthese der Indolalkaloide spielt die analoge Transformation eine bedeutende Rolle. Erste Untersuchungen, die auf dieser Umsetzung basieren, wurden bereits 1911 von A. Pictet und T. Sprengler durchgeführt. Dabei wurde Tryptamin mit einem Aldehyd in Gegenwart von Säuren wie $\mathrm{HCl}$ zur Reaktion gebracht Auf diese Weise gelang der Aufbau von vielen Tetrahydro- $\beta$-carbolin-Derivaten, die zudem durch die Verwendung von chiralen Substituenten bzw. von nachträglich abspaltbaren chiralen Hilfsstoffen stereoselktiv synthetisiert werden konnten. Als Beispiel solch einer 
enantioselektiven Synthese kann hier der Einsatz von chiralen Sulfoxiden zur asymmetrischen Induktion angeführt werden.

Die Synthese des Cyclisierungsproduktes 123 über eine Pictet-Sprengler-Reaktion ist zwar bereits mehrfach im Arbeitskreis von L.F. Tietze durchgeführt worden, allerdings konnten dabei keine zufriedenstellenden Ausbeuten erreicht werden. Erste Versuche, Tryptamin-Hydrochlorid (17) mit der $\alpha$-Ketocarbonsäure 126 in Ethanol zum korrespondierenden Ester 123 umzusetzen, ergaben in Übereinstimmung mit vorherigen Ergebnissen anderer Arbeiten nur Ausbeuten um 55 \%. Die darauffolgende Optimierungsarbeit wurde dahingehend geleistet, indem zum einen mehr Carbonsäure 126 im Vergleich zu den bekannten Synthesevorschriften zur Reaktionslösung nachträglich gegeben wurde, eine Menge von 1.2 Äquivalenten an 126 gewährleistete schliesslich keinen vollständigen Umsatz. Andererseits zeigte der dünnschichtchromatographische Nachweis des Hydrochlorids von 123 in der Mutterlauge, dass dessen Auskristallisation aus dem Reaktionsmedium nur unzureichend stattgefunden hatte. Mehrmaliges Einengen der verbleibenden Lösung führte zum Ausfallen weiteren kristallinen Hydrochlorids und insgesamt zu einer Steigerung der Ausbeute von 123 auf respektable $80 \%$ (Abbildung 53).<smiles>CCOC(=O)CC1NCCc2c1[nH]c1ccccc21</smiles>

17

126

123

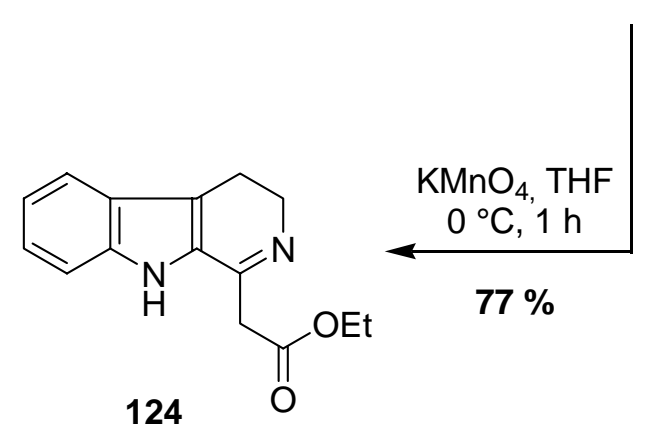

Abbildung 53: Synthese des Imins 124. 
Im nächsten Schritt galt es, in einer optimierten Synthesestrategie den S-konfigurierten Ester 123 und damit den Aldehyd 121 enantiomerenrein aufzubauen. Hierzu sollte eine enantioselektive Reduktion des Imins 124 dienen (Abbildung 54).
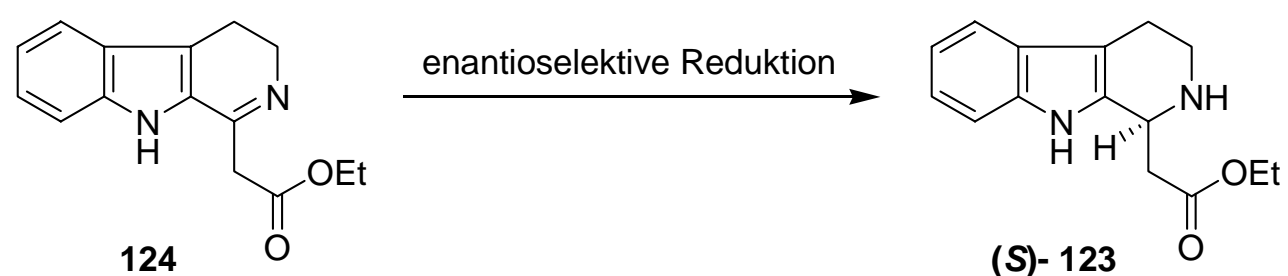

Abbildung 54: Synthese des enantiomerenreinen Aldehyds (1S)-123 aus dem Imin 124.

Grundsätzlich stehen zahlreiche Synthesewege zur Darstellung enantiomerenreiner Tetrahydro- $\beta$-carbolin-Derivate zur Verfügung. Wie bereits kurz dargelegt, sollte in der vorliegenden Arbeit zur Einführung des stereogenen Zentrums C-1 einem katalytischen Prozess der Vorrang gegeben werden. Dies lässt sich damit begründen, dass sich katalytische Verfahren vor allem durch ihre Atomökonomie, Toleranz funktioneller Gruppen sowie der Ökobilanz auszeichnen und dadurch in zunehmendem Masse klassiche Methoden verdrängen. Ein Blick in die Literatur bestättigt diesen Trend. So standen mehrere verschiedene Katalysator-Systeme, die innerhalb der letzten Jahre vorgestellt worden sind, zur Auswahl. Für die Synthese chiraler Amine wurde in der Regel ein gleicher Ansatz verfolgt, der eine asymmetrische Reduktion eines zuvor gebildeten Imins vorsieht. Als vielversprechend hatten sich unter anderem chirale Phosphin-Rhodium- oder Phosphin-IridiumKatalysatoren herausgestellt. Ebenso wurden positive Resultate mit chiralen AnsaTitanocen-Katalysatoren erreicht. Hydrosilylierungen mit den bereits erwähnten Rhodium-Komplexen als auch Hydroborierungen mit chiralen Oxazaborolidinen bewirkten gleichfalls eine asymmetrische Induktion, auch wenn der Enantiomerenüberschuss mit 60-70\% eher bescheiden ausfiel. Geeigneter schien daher die bereits im Jahre 1996 von $R$. Noyori entwickelte Transferhydrierung mit $\mathrm{HCO}_{2} \mathrm{H}_{/} \mathrm{NEt}_{3}$ als Wasserstoff-Quelle in Gegenwart von chiralen Ruthenium-Katalysatoren. 
Zunächst war es notwendig, für die asymmetrische Transferhydrierung das Imin 124 bereitzustellen. Eine simple Oxidation des racemischen Tetrahydro- $\beta$-carbolinDerivates 123 mit $\mathrm{KMnO}_{4}$ bei $0{ }^{\circ} \mathrm{C}$ lieferte nach nur einer Stunde die gewünschte Verbindung 124. Die Aubeuten variierten in Abhängigkeit der eingesetzten Menge am Edukt 123. Als limitierender Faktor stellte sich Braunstein heraus, welches im Laufe der Reaktion als Folgeprodukt der Reduktion von Kaliumpermanganat gebildet wird und das Imin $124 \mathrm{zu}$ absorbieren vermag. Grössere Ansätze von mehr als $10 \mathrm{~g}$ resultierten so in nur mäßigen Ausbeueten. Das beste Ergebnis wurde durch den Umsatz von $1 \mathrm{~g}$ Ester 123 mit $10 \mathrm{~g} \mathrm{KMnO}_{4}$ mit einer Ausbeute von 77 \% erzielt.

Für die beabsichtigte asymmetrische Transferhydrierung standen zwei chirale Ruthenium-Katalysatoren zur Verfügung, die sich lediglich in der Struktur eines Substituenten unterscheiden. In der Arbeitsgruppe von Noyori wurden die Reaktionsbedingungen dieser Umsetzung detailliert untersucht. Bei der Verwendung diverser Ruthenium-Komplexe erwies sich insbesondere 125 als potenter Katalysator für die Reduktion von Iminen mit einem Tetrahydro- $\beta$-carbolin-Grundgerüst. Dagegen hat sich 159 zur Synthese enantiomerenreiner Isochinolin-Derivate etabliert.

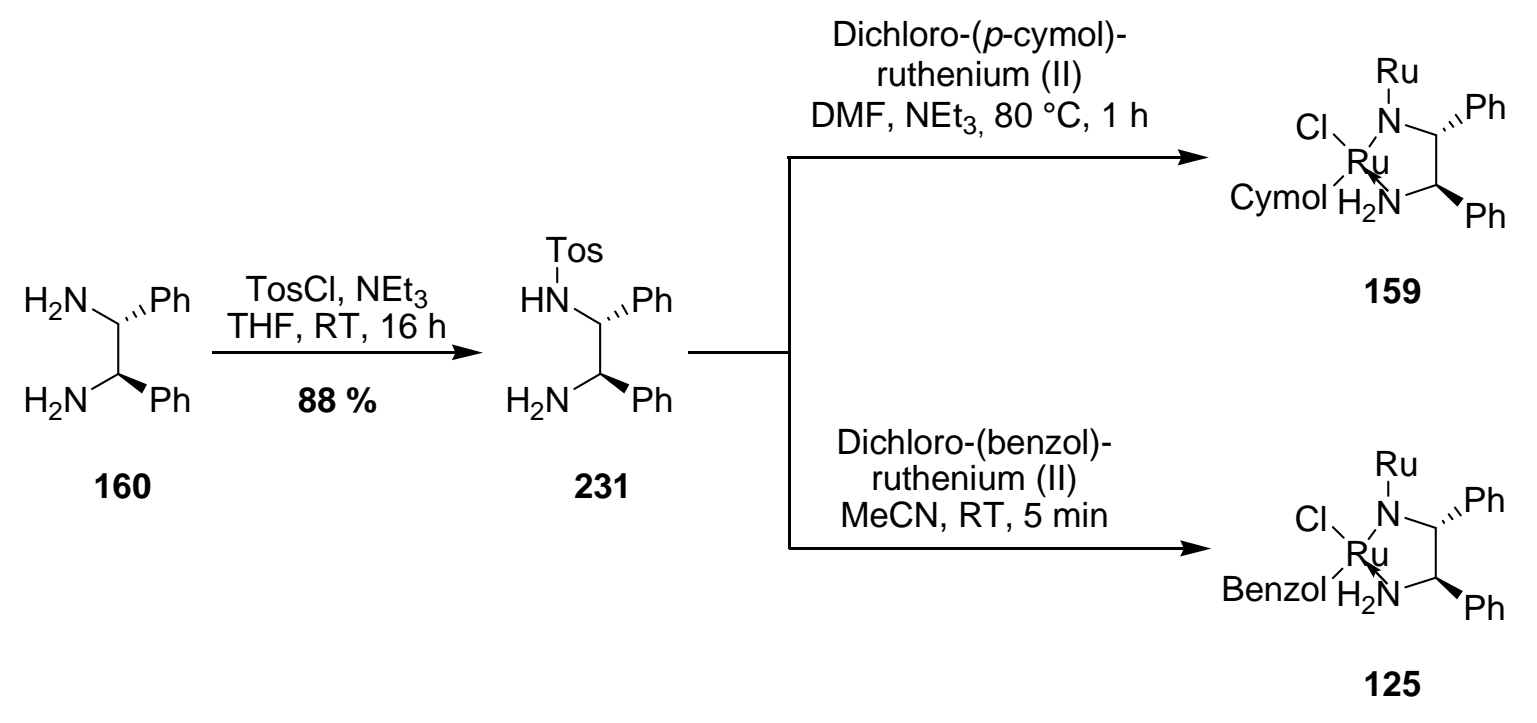

Abbildung 55: Synthese der chiralen Katalysatoren 159 und 125 für die asymmetrische Transferhydrierung. 
Mit welchem Katalysator der Weg letztendlich erfolgreich beschritten werden kann, sollte für das vorliegende System eingehend experimentell erprobt werden.

Die Tosylierung des käuflichen Diamins $(R, R)-\mathbf{1 6 0}$ mit Tosylchlorid wurde in THF durchgeführt und ergab nach $16 \mathrm{~h}$ bei Raumtemperatur den chiralen Liganden $(R, R)-\mathbf{1 6 1}$ in sehr guter Ausbeute (Abbildung 55). Nachfolgende in situ Generierung der Katalysatoren fand unter den in Abbildung 55 beschriebenen Reaktionsbedingungen statt. Anschließend sollte die asymmetrische Induktion des Imins 124 untersucht werden. Zunächst wurde versucht, den enantiomeren Ester 123 unter den von $Y$. Zhou verwendeten Reaktionsbedingungen zu synthetisieren.

Die in Tabelle 3 zusammen-gefassten Befunde erzielten nicht den erhofften Durchbruch, so dass weitere Optimierung notwendig war. Hierzu sollte der von N. Rackelmann verwendete Katalysator 159 zum Einsatz kommen, mit dem zuvor mit sehr guten Ausbeuten und Selektivitäten von $93 \%$ bzw. $>95 \%$ ee das enantiomerenreine Isochinolin-System 163 synthetisch zugänglich gemacht werden konnte (Abbildung 56).

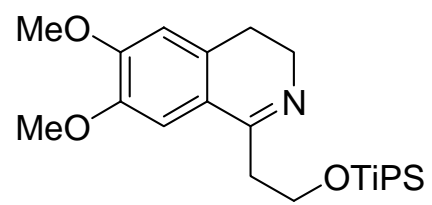

162

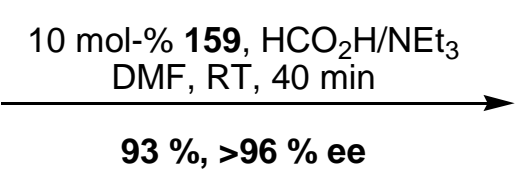

$93 \%,>96 \%$ ee

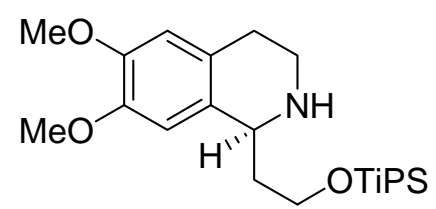

163

Abbildung 56: Synthese des enantiomerenreinen Isochinolin-Derivates (1S)-163 von N. Rackelmann.

Wie bereits kurz erwähnt, gestaltete sich die asymmetrische Transferhydrierung mit dem Phenyl-substituierten Katalysator 125 entgegen den Literaturangaben als auch den im Arbeitskreis Tietze durchgeführten Versuchsreihen als unerwartet schwierig. Mit Acetonitril als Lösungsmittel und 2 mol\% des Katalysators 125 konnte nach 5 Tagen nur eine mässige Ausbeute von $8 \%$ verzeichnet werden. Erhöhung der Katalysatormenge um das 5-fache ergab ebenso keinen nennenswerten Anstieg der Ausbeute. Verantwortlich dafür lässt sich wohl eine zu geringe Konzentration der Reaktionslösung ausmachen. Aus diesem Grund wurde dieser Ansatz bei einer 
höheren Konzentration als auch mit einer erhöhten Katalysatormenge wiederholt. Diese Vorgehensweise führte zu weitaus besseren Ausbeuten, allerdings stellten sich die Enantiomerenüberschüsse als nicht reproduzierbar heraus (siehe Tabelle 3).

\begin{tabular}{|c|c|c|c|c|}
\hline Nr. & Katalysator & Reaktionsbedingungen & $\begin{array}{l}\text { Ausbeute } \\
\text { [\%] }\end{array}$ & $\begin{array}{c}e e \\
{[\%]}\end{array}$ \\
\hline 1 & $2 \mathrm{~mol} \% 125$ & 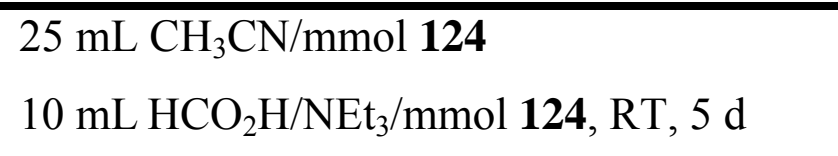 & 8 & - \\
\hline 2 & $10 \mathrm{~mol} \% 125$ & 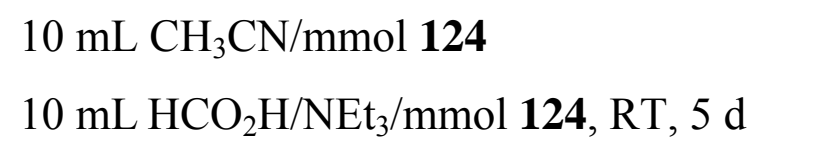 & 21 & - \\
\hline 3 & $20 \mathrm{~mol} \% 125$ & $\begin{array}{l}2 \mathrm{~mL} \mathrm{CH}{ }_{3} \mathrm{CN} / \mathrm{mmol} 124 \\
0.5 \mathrm{~mL} \mathrm{HCO} \mathrm{HCO}_{2} \mathrm{H} / \mathrm{NEt}_{3} / \mathrm{mmol} \mathrm{124}, \mathrm{RT}, 42 \mathrm{~h}\end{array}$ & 98 & 69 \\
\hline 4 & $10 \mathrm{~mol} \% 125$ & 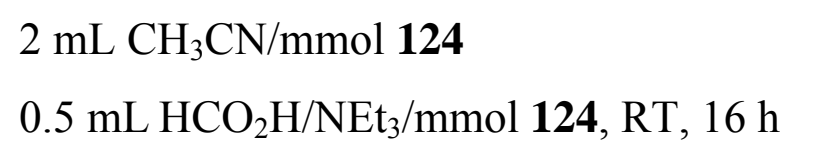 & 91 & 84 \\
\hline 5 & $10 \mathrm{~mol} \% 125$ & 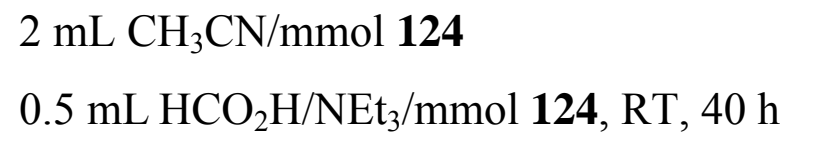 & 79 & \\
\hline 6 & $10 \mathrm{~mol} \% 125$ & 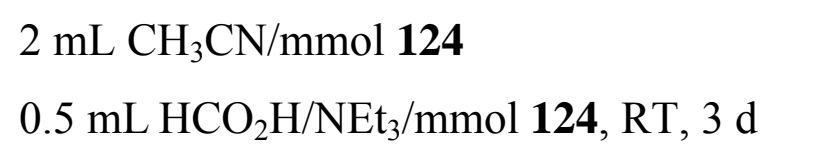 & 51 & \\
\hline 7 & $10 \mathrm{~mol} \% 125$ & $\begin{array}{l}2 \mathrm{~mL} \mathrm{DMF} / \mathrm{mmol} 124 \\
0.5 \mathrm{~mL} \mathrm{HCO} \mathrm{H}_{2} / \mathrm{NEt}_{3} / \mathrm{mmol}^{124}, \mathrm{RT}, 5 \mathrm{~d}\end{array}$ & $\begin{array}{l}\text { keine } \\
\text { Rkt. }\end{array}$ & - \\
\hline 8 & $10 \mathrm{~mol} \% 125$ & 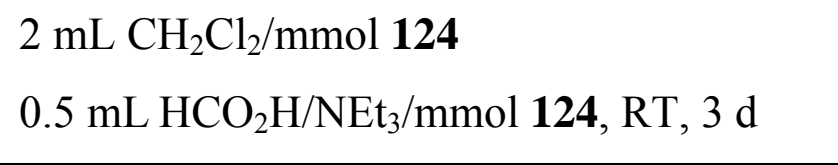 & $\begin{array}{l}\text { kaum } \\
\text { Umsatz }\end{array}$ & - \\
\hline 9 & $10 \mathrm{~mol} \% 159$ & 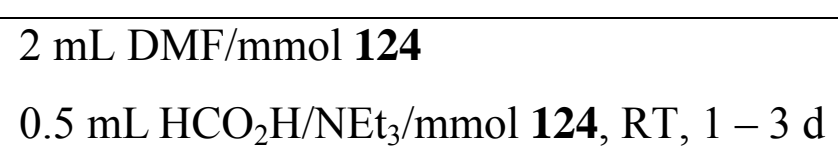 & $23-45$ & \\
\hline 10 & $10 \mathrm{~mol} \% 159$ & $\begin{array}{l}2 \mathrm{~mL} \mathrm{CH}{ }_{3} \mathrm{CN} / \mathrm{mmol} 124 \\
0.5 \mathrm{~mL} \mathrm{HCO}{ }_{2} \mathrm{H} / \mathrm{NEt}_{3} / \mathrm{mmol} \mathrm{124} \mathrm{RT}, 16 \mathrm{~h}\end{array}$ & 83 & \\
\hline 11 & $10 \mathrm{~mol} \% 159$ & $\begin{array}{l}2 \mathrm{~mL} \mathrm{CH} \mathrm{Cl}_{2} / \mathrm{mmol} 124 \\
0.5 \mathrm{~mL} \mathrm{HCO} \mathrm{HCt}_{2} \mathrm{NEt} / \mathrm{mmol} \mathrm{124,} \mathrm{RT,} 2 \mathrm{~d}\end{array}$ & $\begin{array}{l}\text { keine } \\
\text { Rkt. }\end{array}$ & - \\
\hline
\end{tabular}

Tabelle 3: Versuche zur Optimierung der asymmetrischen Transferhydrierung. 
Entscheidender war, dass sich diese Resultate nicht auf grössere Reaktionsansätze übertragen liessen. Auch die Modifikation des Lösungsmittels ergab keine Verbesserung hinsichtlich der Ausbeuten und der ee-Werte. Schließlich wurde auf ein weiteres katalytisches System zurückgegriffen, welches in der Vergangenheit erfolgreich bei Isochinolinen eingesetzt worden ist. Trotz mehrmaliger Versuche unter verschiedenen Reaktionsbedingungen, gelang es jedoch auch mit dem Katalysator 159 nicht eine Verbesserung insbesondere der ee-Werte zu erzielen.

Es wurde ebenso untersucht, ob in einer alternativen Synthesestrategie, die von den geschützten Alkoholen 167 und 168 anstatt vom Ester 124 ausging, die Einführung des stereogenen Zentrums mittels der Noyori-Reduktion möglich war. Für diesen Umweg wurde die Ausgangsverbindung 123 zunächst mit Lithiumaluminiumhydrid zum korrespondierenden Alkohol 164 in hohen Ausbeuten von 88 \% reduziert und dieser als TiPS- bzw. TBS-Ether geschützt (Abbildung 57). Die Oxidation mit Kaliumpermanganat lieferte dann die gewünschten Substrate 167 und 168 für die geplante asymmetrische Transferhydrierung. Allerdings konnten auch in diesen Fällen die gewünschten enantiomerenreinen Verbindungen 165 und 166 nicht erhalten werden.

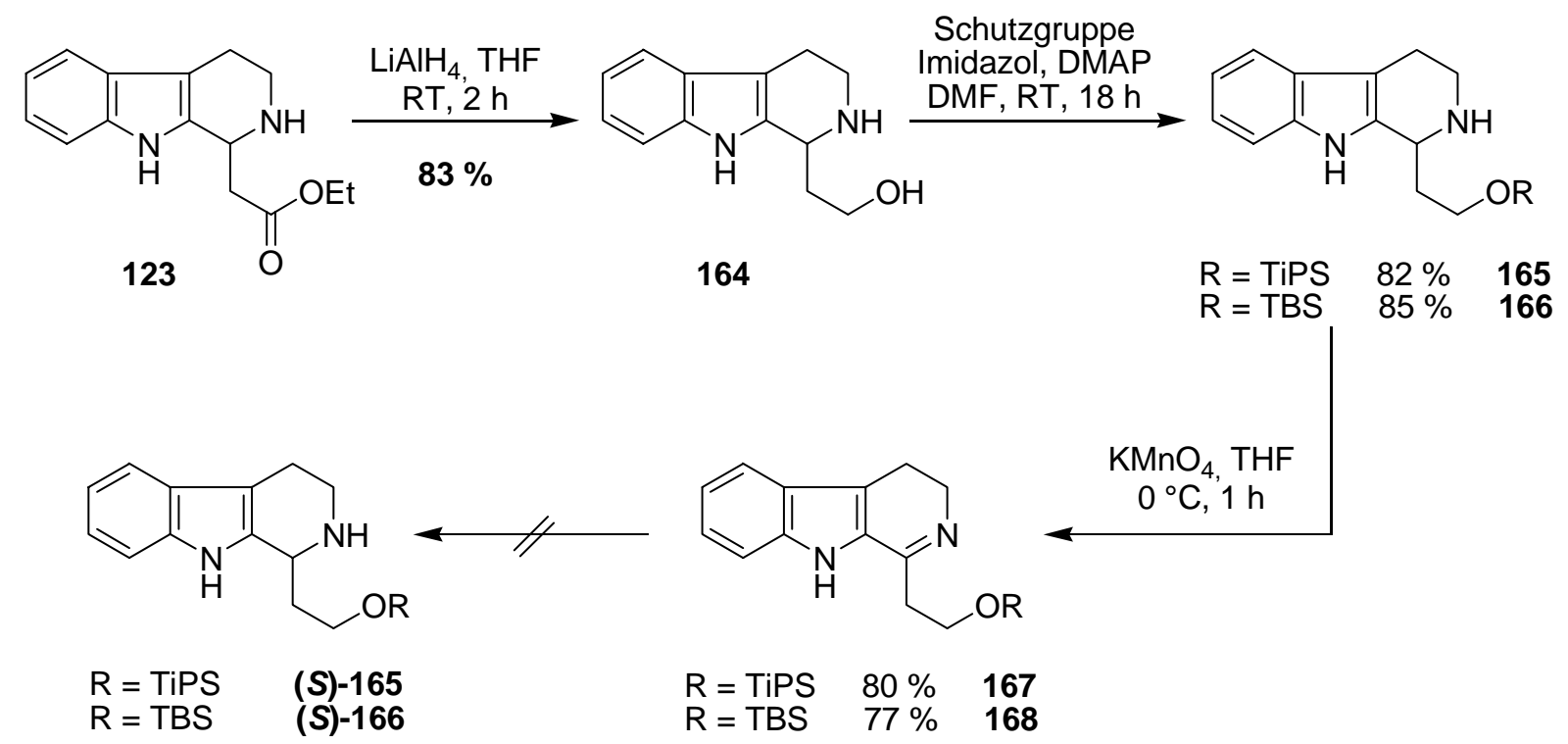

Abbildung 57: Versuche zur Synthese der enantiomerenreinen Silylether (1S)-165 und (1S)-166. 
Daraufhin wurde die Durchführbarkeit der Noyori-Hydrierung eingehend an einem literaturbekannten Modellsystem untersucht.

Zunächst wurde das Ethylamin (169) zu 170 acyliert und konnte nach einigen Modifikationen der Synthesevorschrift in $86 \%$ Ausbeute gewonnen werden. Anschließend wurde dieses in einer Bischler-Napieralski-Isochinolin-Synthese eingesetzt (Abbildung 58).<smiles>COc1ccc(CCN)cc1OC</smiles>

169

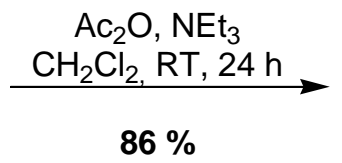

(N)

172

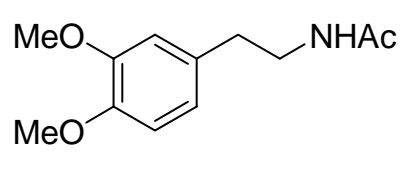

170
$\mathrm{POCl}_{3}$, Toluol Reflux, $2 \mathrm{~h}$ $83 \%$

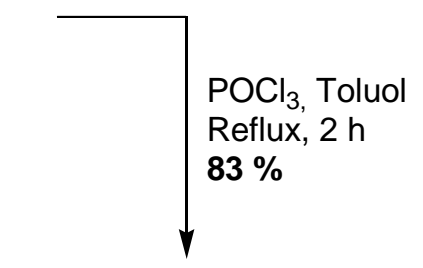<smiles>COc1cc2c(cc1OC)C(C)=NCC2</smiles>

171

Abbildung 58: Modellsystem für die asymmetrische Transferhydrierung.

Das erhaltene Dihydroisochinolin 171 wurde dann unter bekannten Reaktionsbedingungen mit 0.2 mol\% des Katalysators 159 umgesetzt, was allerdings nur eine Reisolierung des Eduktes 171 zur Folge hatte.

\begin{tabular}{ccccc}
\hline Nr. & $\begin{array}{c}\text { Katalysator 159 } \\
{[\mathbf{m o l} \%]}\end{array}$ & Reaktionsbedingungen & $\begin{array}{c}\text { Ausbeute } \\
{[\%]}\end{array}$ & $\begin{array}{c}\text { ee } \\
\text { [\%] }\end{array}$ \\
\hline 1 & 0.2 & $\mathrm{HCO}_{2} \mathrm{H} / \mathrm{NEt}_{3}=5: 2$ & Reisolierung von & - \\
& & $\mathrm{CH}_{3} \mathrm{CN}, \mathrm{RT}, 17 \mathrm{~h}$ & $\mathbf{1 7 1}$ & \\
2 & 10.0 & $\mathrm{HCO}_{2} \mathrm{H} / \mathrm{NEt}_{3}=5: 2$ & 81 & \\
& & $\mathrm{CH}_{3} \mathrm{CN}, \mathrm{RT}, 17 \mathrm{~h}$ & & \\
\hline
\end{tabular}

Tabelle 4: Versuche zur Optimierung der asymmetrischen Transferhydrierung am Modellsystem. 
Die Erhöhung der Katalysatormenge auf $10 \mathrm{~mol} \%$ führte zwar zu der gewünschten Zielverbindung 172 in sehr guten Ausbeuten von $81 \%$, leider aber auch zu sehr niedrigen Enantiomerenüberschüssen. Das offensichtliche Problem der manchmal unzureichenden Reproduzierbarkeit der asymmetrischen Transferhydrierung nach Noyori wurde bereits in den Arbeiten von Abraham beschrieben.

Aufbauend auf diesen Ergebnissen wurde daraufhin die enantioselektive Reaktionsführung zunächst aufgegeben und der Aldehyd 121 in racemischer Form aufgebaut. Hierbei wurde Tryptamin-Hydrochlorid (17) mit der Ketosäure 126 unter den zuvor optimierten Bedingungen zum Carbolin 123 umgesetzt. Die nachfolgende selektive Schützung des sekundären Stickstoff-Atoms mit $\mathrm{CbzCl}$ gefolgt von Reduktion des Esters 173 mit Diisobutylaluminiumhydrid führte zum gewünschten Produkt 121 und somit zu dem zweiten Baustein für die Domino-Knoevenagel-heteroDiels-Alder-Reaktion.

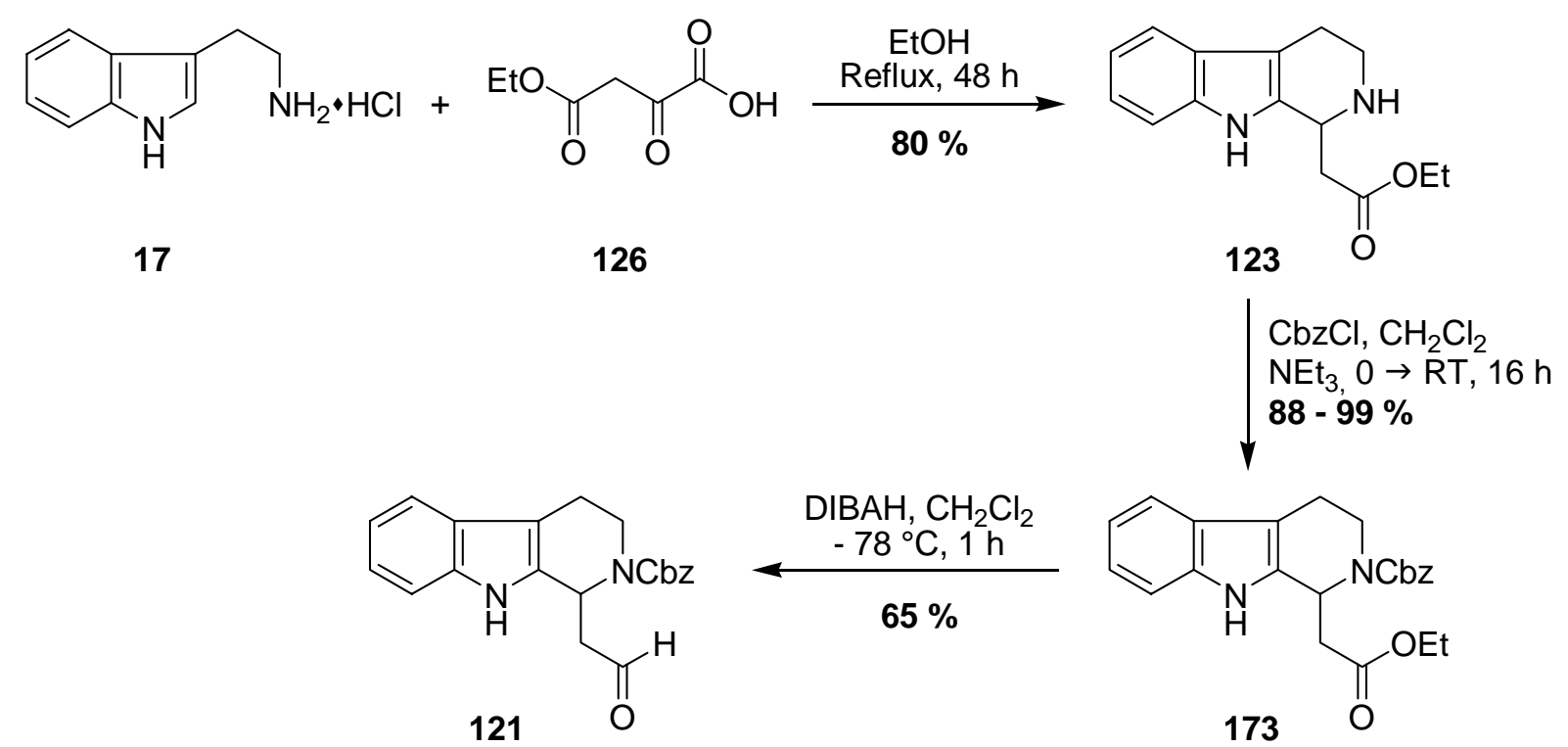

Abbildung 59: Synthese des racemischen Aldehyds 121 für die Domino-Knoevenagel-hetero-Diels-AlderReaktion.

Zusätzlich und in Hinblick auf eine stereoselektive Reaktionsführung bei dem Domino-Knoevenagel-hetero-Diels-Alder-Prozess sollte der Carbolin-Aldehyd 121 in 
einer $N$-Boc-geschützten Form synthetisiert werden. Hierfür wurde der Ester 173 zuerst einer Boc-Schützung und dann einer DIBAH-Reduktion zum entsprechenden Aldehyd 118 unterzogen (Abbildung 60).<smiles>CCOC(=O)CC1c2[nH]c3ccccc3c2CCN1CC</smiles>

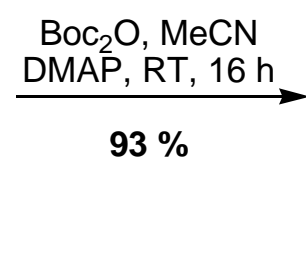<smiles>CCOC(=O)CC1C2=C(CCN1C)c1ccccc12</smiles>

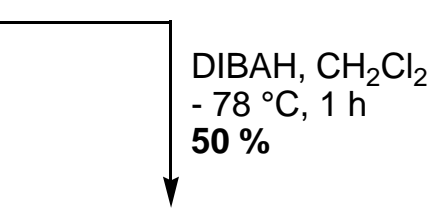<smiles>O=CCC1c2[nH]c3ccccc3c2CCN1C(=O)O</smiles>

Abbildung 60: Synthese des Aldehyds 118.

\subsection{Diskussion der spektroskopischen Daten der Verbindung 174}

\section{UV-, IR- und Massenspektren}

Das UV-Spektrum des Esters 174 zeigt neben dem Absorbtionsmaximum bei $\lambda=228.5 \mathrm{~nm}$ zwei weitere Banden bei $\lambda=266.0$ und $293.0 \mathrm{~nm}$.

Im IR-Spektrum sieht man eine starke Doppelbande bei 1732 und $1699 \mathrm{~cm}^{-1}$, die auf die Absorbtion der $\mathrm{C}=\mathrm{O}$-Streckschwingungen des Ethylesters und der beiden UrethanKomponenten zurückzuführen ist. Die Streckschwingungen der aromatischen Protonen zeigen sich bei 3034 und $2978 \mathrm{~cm}^{-1}$. Weitere auffällige Banden bei 1456, 1423 und $1323 \mathrm{~cm}^{-1}$ sind den C-H-Deformationsschwingungen der Methylen- und Methylgruppen zuzuordnen. Zwei starke Absorptionen bei 751 und $698 \mathrm{~cm}^{-1}$ lassen auf die out of plane-Schwingungen der aromatischen Protonen zurückführen.

Im Massenspektrum von 174 wird der Basispeak vom Tropylium-Kation bei $\mathrm{m} / \mathrm{z}=91$ gebildet, welches aus der schrittweisen Abspaltung der Cbz-Schutzgruppe entsteht. Die zweitstärkste Intensität weist der Peak auf, dem das tert-Butyl-Kation bei $\mathrm{m} / \mathrm{z}=57$ 
zuzuordnen ist, das aus der Boc-Gruppe resultiert. Infolge der Abspaltung der Schutzgruppen ist die Intensität des Molekülions bei $m / z=492$ nur schwach.

\section{NMR-Spektren}

Die NMR-Spektren der Verbindung 174 sind durch das Auftreten von Rotameren etwa gleicher Intensität gekennzeichnet. So erscheinen im ${ }^{1}$ H-NMR-Spektrum die Signale der drei chemisch äquivalenten Methylgruppen der tert-Butylgruppe als zwei Singuletts bei $\delta=1.59$ und 1.72 im Intensitätsverhältnis von etwa 6:5. Für 1-H findet man zwei Dubletts mit $J=8.0 \mathrm{~Hz}$ bei $\delta=6.25$ und 6.35. Das äquatoriale Proton an C-3 resoniert als zwei Dubletts von Dubletts mit $J=13.2$ und $5.4 \mathrm{~Hz}$ bei $\delta=4.38$ und 4.52. Die beiden Rotamere sind isochron, so dass im Aromatenbereich kein doppelter Signalsatz in Erscheinung tritt. Die Substitution an $N-9$ mit Boc führt zu einer Tieffeldverschiebung von 8-H als Dublett mit $J=7.2 \mathrm{~Hz}$ bei $\delta=8.16$.

Im ${ }^{13} \mathrm{C}$-NMR-Spektrum führt das Auftreten von Rotameren bei einigen Kohlenstoffatomen $\mathrm{zu}$ einem doppelten Signalsatz. Die Ester-Carbonylgruppe resoniert bei $\delta=169.9$ und 170.1. Die Signale des C- 1 sind bei $\delta=49.85$ und die des C-3 bei $\delta=36.35$ und $39.10 \mathrm{zu}$ finden . 


\section{Die Domino-Knoevenagel-hetero-Diels-Alder-Reaktion}

\subsection{Disskussion des Reaktionsmechanismus}

Für den Domino-Prozess wird folgender Mechanismus angenommen:

Im ersten Schritt findet eine durch Ethylendiammoniumdiacetat katalysierte Kondensation der Meldrum-Säure (108) mit dem enantiomerenreinen Aldehyd 118 bzw. 121 statt. Das dabei gebildete 1-Oxa-1,3-butadien 176 bzw. 128 liegt aufgrund fehlender sterischer oder elektronischer Wechselwirkungen sowie freier Drehbarkeit um die Einfachbindung in zwei nahezu gleichberechtigten Konformeren 176a/128a bzw. 176a/128b vor. Die zuvor bei Versuchen von N. Rackelmann beobachtete und auch in diesem Fall zu erwartende fehlende Selektivität der folgenden hetero-DielsAlder-Reaktion mit inversem Elektronenbedarf könnte an dieser Flexibilität des Systems liegen, wenn man annimmt, dass der Angriff des Dienophils 122 von der sterisch gehinderten Seite auf das Konformer 175a/128a bzw. 175b/128b stattfindet. Das Konformer 175a/128a sollte dann das primäre Cycloaddukt 176a/177a mit den H-Atomen anti bezüglich C-1 und C-4' zueinander und das Konformer 176b/128b das Cycloaddukt 176b/177b mit den H-Atomen cis bezüglich C-1 und C-4' zueinander bilden. Eine andere Erklärung für die mangelnde Selektivität könnte der fehlende Einfluss des Stereozentrums an C-1 auf den Angriff des Dienophils 122 sein. Die Reaktion fände dann ohne faciale Differenzierung statt. Experimente aus der Arbeit von N. Rackelmann legen nahe, dass die nicht vorhandene Fixiertheit des Systems als Grund für die fehlende Selektivität wahrscheinlich ist als ein Angriff ohne faciale Differenzierung. Der stereoselektive Aufbau von C-2' und C-3' ist nicht zu erwarten, da nur Domino-Knoevenagel-hetero-Diels-Alder-Reaktionen, bei denen die heteroDiels-Alder-Reaktion intramolekular verläuft, gute bis sehr gute endo/exoSelektivitäten erzielt werden und das Dienophil zusätzlich noch als E/ZIsomerengemisch eingesetzt wurde. Das Stereozentrum an Position C-2' sollte im weiteren Reaktionsverlauf ohnehin wieder aufgehoben werden, sodass eine Kontrolle diesbezüglich nicht erforderlich ist. 


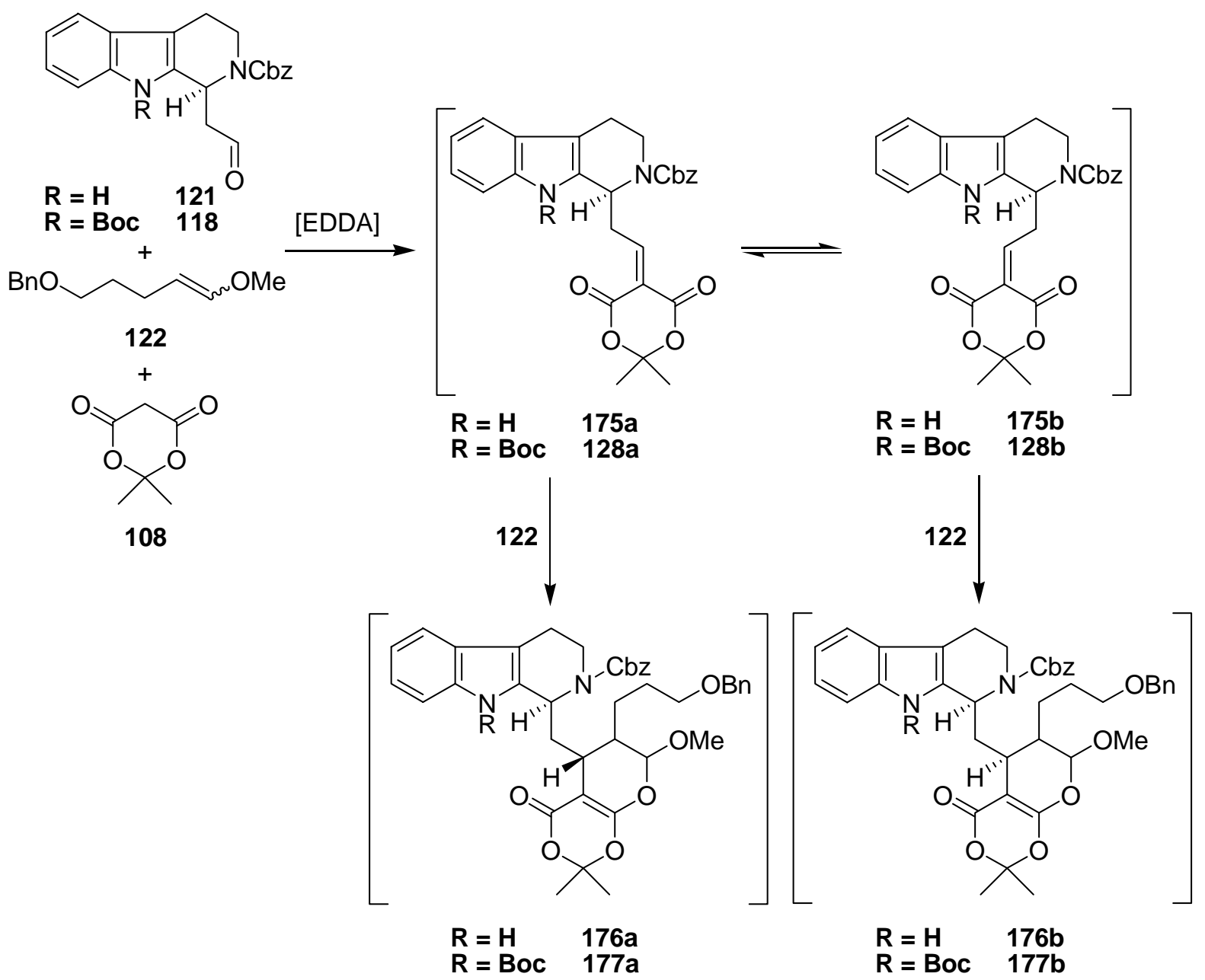

Abbildung 61: Reaktionsmechanismus der Domino Knoevenagel-hetero-Diels-Alder-Reaktion.

Für die Bildung von 120/178 aus 176a/177a und 176b/177b stehen nun zwei unterschiedliche Reaktionskanäle offen (Abblidung 62).

Zum einen könnte 176a/177a bzw. 176b/177b in einer retro-Diels-Alder-Reaktion unter Freisetzung von Aceton zum Keten 181/182 reagieren. In einer folgenden Addition von $\mathrm{H}_{2} \mathrm{O}$, das bei der Knoevenagel-Kondensation frei wird, könnte es dann zur Carbon-säure 179/180 reagieren. Alternativ könnten die Acetonide 176a/177a bzw. 176b/177b mit Wasser zu 179/180 reagieren, welches dann zu 120/178 decarboxyliert wird. 


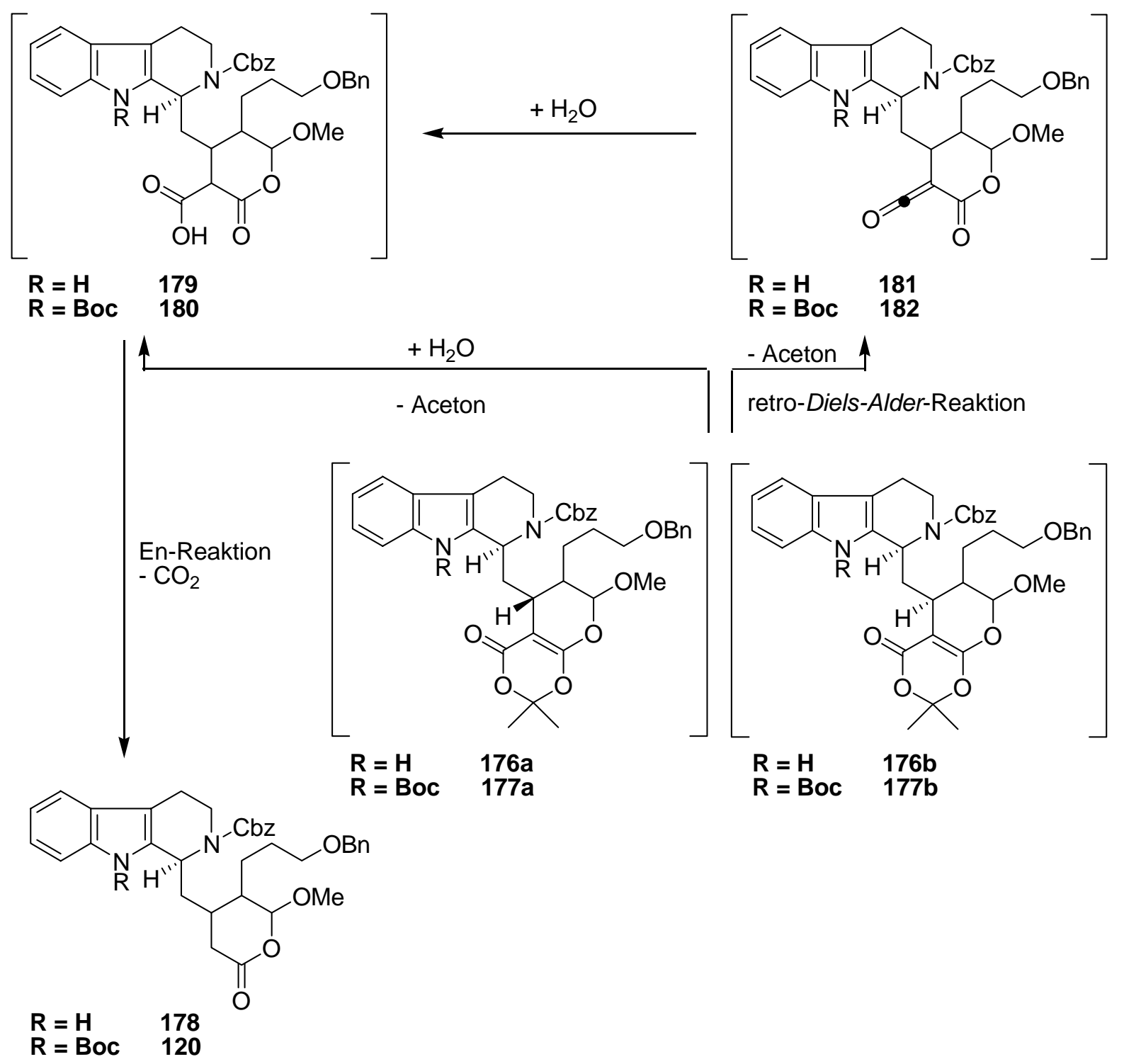

Abbildung 62: Bildung des Endprodukts 118/179 der Domino Knoevenagel-hetero-Diels-Alder-Reaktion.

\subsection{Die biomimetische Cyclisierung}

Für die biomimetische Cyclisierung wird folgender Mechanismus angenommen:

Das Lactonacetal 120/178 wird durch Methanol in Gegenwart von $\mathrm{K}_{2} \mathrm{CO}_{3}$ unter Bildung eines Carbonsäuremethylesters und eines Halbacetals, das anschließend zum Aldehyd 183/184 zerfällt, geöffnet. 


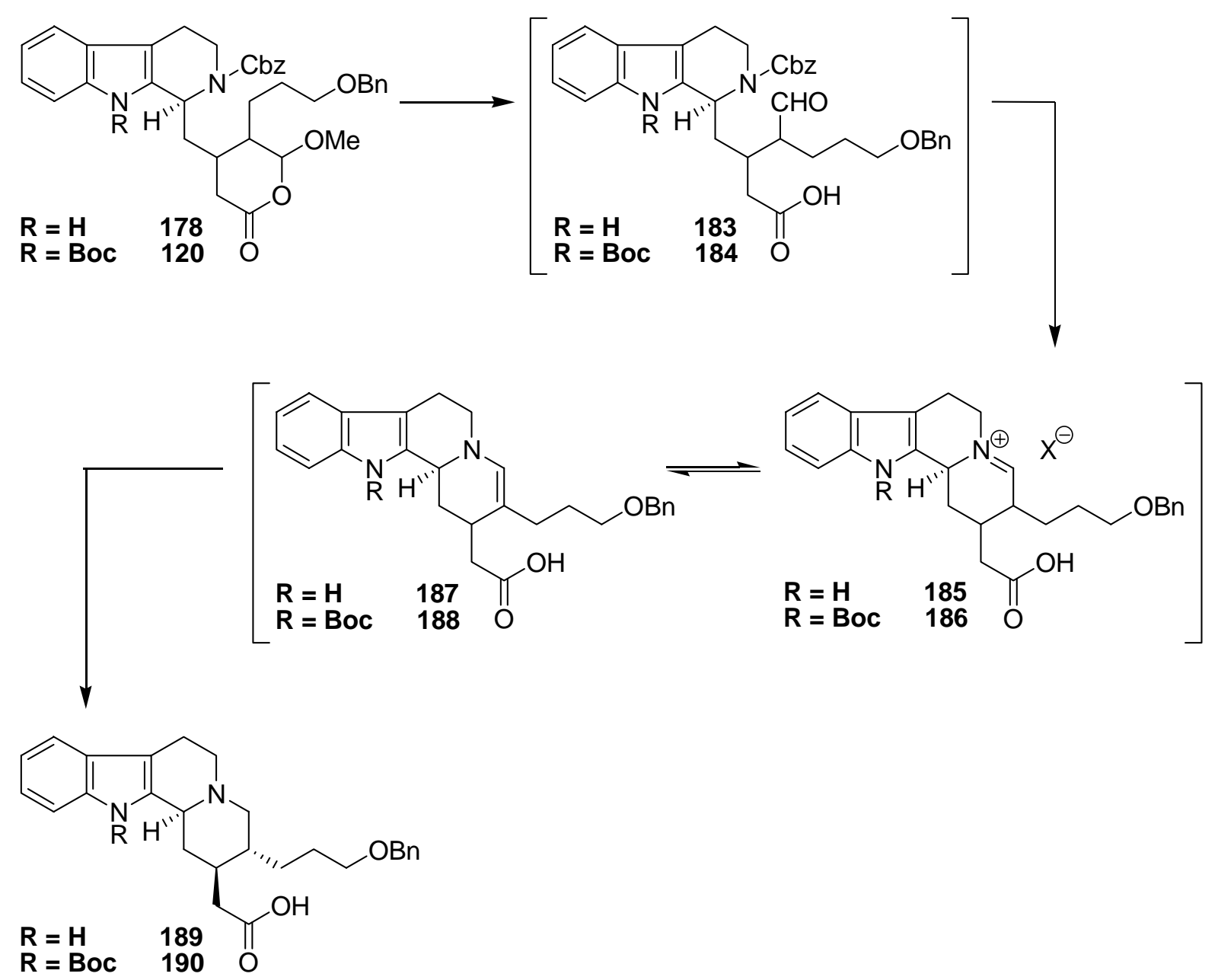

Abbildung 63: Mechanismus der biomimetischen Cyclisierung.

Nach hydrogenolytischer Abspaltung der Schutzgruppe (Cbz) am Stickstoff des Pyrans kondensiert der Aldehyd 183/184 intramolekular mit dem Stickstoffatom zu einem Tetrazyklus. Unter basischen Bedingungen kann das entstandene Iminiumion 185/186 zum Enamin 187/188 tautomerisieren, das unter den Reaktionsbedingungen hydriert wird. Allerdings besteht auch die Möglichkeit, dass das primär gebildete Iminiumion 185/187 direkt hydriert wird.

Bei Verwendung von 120 mit $\mathrm{R}$ = Boc war nur ein Diastereomer zu erwarten, da im Übergangszustand das 1-Oxa-1,3-butadien aufgrund sterischer Wechselwirkung mit der tert-Butoxycarbonyl-Gruppe am Indol-Stickstoff fixiert ist. 


\subsection{Untersuchungen zur Domino-Knoevenagel-hetero-Diels-Alder-Reaktion}

Mit Hilfe der hochkonvergenten drei Komponenten Domino-Knoevenagel-heteroDiels-Alder-Reaktion und einem anschließenden zweiten biomimetischen DominoProzess sollte nun das Grundgerüst 18 des Yohimbins (9) aufgebaut werden. Hierzu wurden die racemischne Aldehyde 121 und 118 mit Meldrum-Säure (108), dem Enolether 122 als Dienophil und einer katalytischen Menge EDDA versetzt und in wenig Benzol gelöst. Die Reaktion wurde in einem Druckkolben im Ultraschall-Bad durchgeführt.

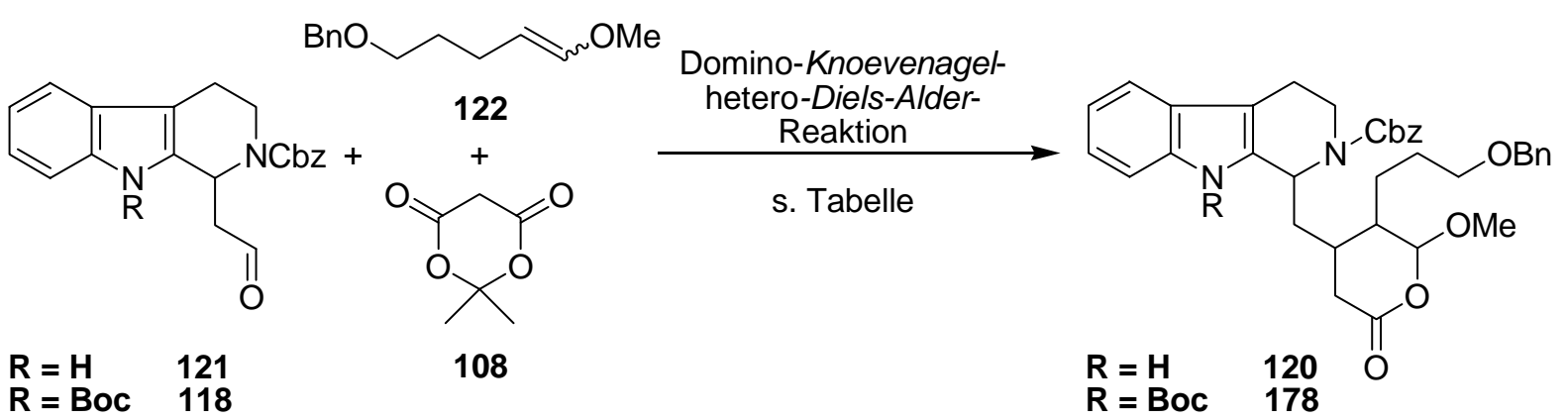

Abbildung 64: Domino Knoevenagel-hetero-Diels-Alder-Reaktion.

Im Fall der nicht Boc-geschützten Verbindung 118 wurde das gewünschte Produkt 178 zunächst in $12 \%$ Ausbeute als Diastereomerengemisch erhalten, das auf dieser Stufe der Synthesesequenz allerdings nicht getrennt werden konnte. Weitere Versuche führten jedoch trotz umfangreicher Optimierungsversuche nur $\mathrm{zu}$ komplexen Gemischen aufgrund der offensichtlichen Instabilität der Pyrane 120/178. Lediglich eine Beschleunigung der Umsetzung bei höhreren Temperaturen konnte festgestellt werden. 


\begin{tabular}{|c|c|c|c|}
\hline Nr. & Rest $\mathbf{R}$ & Reaktionsbedingungen & Ausbeute [\%] \\
\hline 1 & $\overline{\mathrm{H}}$ & $\begin{array}{l}1.2 \text { Äq. Meldrum-Säure, } 3.0 \text { Äq. } 122 \\
\text { kat. EDDA, Benzol, RT, } 2 \text { d, Rühren }\end{array}$ & keine Umsetzung \\
\hline 2 & $\mathrm{H}$ & $\begin{array}{l}1.2 \text { Äq. Meldrum-Säure, } 3.0 \text { Äq. } 122 \\
\text { kat. EDDA, Benzol, } 60^{\circ} \mathrm{C}, 7 \text { h, Rühren }\end{array}$ & $\begin{array}{l}\text { nächste } \\
\text { Transformation }\end{array}$ \\
\hline 3 & $\mathrm{H}$ & $\begin{array}{l}1.2 \text { Äq. Meldrum-Säure, } 3.0 \text { Äq. } 122 \\
\text { kat. EDDA, Benzol, } 60^{\circ} \mathrm{C}, 7 \text { h, Ultraschall }\end{array}$ & 12 \\
\hline 4 & $\mathrm{H}$ & $\begin{array}{c}1.2 \text { Äq. Meldrum-Säure, } 3.0 \text { Äq. } 122 \\
\text { kat. EDDA, Benzol, } 60^{\circ} \mathrm{C}, 7 \text { h, Ultraschall }\end{array}$ & Zersetzung \\
\hline 5 & $\mathrm{H}$ & $\begin{array}{l}1.2 \text { Äq. Meldrum-Säure, } 3.0 \text { Äq. } 122 \\
\text { kat. EDDA, Benzol, } 60^{\circ} \mathrm{c}, 16 \text { h, Ultraschall }\end{array}$ & Multispot-Reaktion \\
\hline 6 & $\mathrm{H}$ & $\begin{array}{c}1.2 \text { Äq. Meldrum-Säure, } 1.5 \text { Äq. } 122 \\
\text { kat. EDDA, Benzol, } 60{ }^{\circ} \mathrm{C}, 16 \text { h, Ultraschall }\end{array}$ & Multispot-Reaktion \\
\hline 7 & $\mathrm{H}$ & $\begin{array}{c}1.2 \text { Äq. Meldrum-Säure, } 1.5 \text { Äq. } 122 \\
\text { kat. EDDA, Benzol, } 60^{\circ} \mathrm{C}, 5 \text { h, Ultraschall }\end{array}$ & Multispot-Reaktion \\
\hline 8 & $\mathrm{H}$ & $\begin{array}{l}1.2 \text { Äq. Meldrum-Säure, } 1.5 \text { Äq. } 122 \\
\text { kat. EDDA, Benzol, } 80^{\circ} \mathrm{C}, 4 \text { h, Ultraschall }\end{array}$ & Multispot-Reaktion \\
\hline 9 & $\mathrm{H}$ & $\begin{array}{l}1.2 \text { Äq. Meldrum-Säure, } 3.0 \text { Äq. } 122 \\
\text { kat. EDDA, Benzol, } 80^{\circ} \mathrm{C}, 4 \text { h, Ultraschall }\end{array}$ & Multispot-Reaktion \\
\hline 10 & $\mathrm{H}$ & $\begin{array}{l}1.2 \text { Äq. Meldrum-Säure, } 3.0 \text { Äq. } 122 \\
\text { kat. EDDA, Benzol, } 90^{\circ} \mathrm{C}, 4 \text { h, Ultraschall }\end{array}$ & Gemisch \\
\hline 11 & Boc & $\begin{array}{l}1.2 \text { Äq. Meldrum-Säure, } 3.0 \text { Äq. } 122 \\
\text { kat. EDDA, Benzol, RT, } 2 \text { d, Rühren }\end{array}$ & keine Umsetzung \\
\hline 12 & Boc & $\begin{array}{l}1.2 \text { Äq. Meldrum-Säure, } 3.0 \text { Äq. } 122 \\
\text { kat. EDDA, Benzol, } 60^{\circ} \mathrm{C}, 7 \text { h, Rühren }\end{array}$ & $\begin{array}{l}\text { nächste } \\
\text { Transformation }\end{array}$ \\
\hline 13 & Boc & $\begin{array}{c}1.2 \text { Äq. Meldrum-Säure, } 3.0 \text { Äq. } 122 \\
\text { kat. EDDA, Benzol, } 60^{\circ} \mathrm{C}, 7 \text { h, Ultraschall }\end{array}$ & Gemisch \\
\hline 14 & Boc & $\begin{array}{l}1.2 \text { Äq. Meldrum-Säure, } 1.5 \text { Äq. } 122 \\
\text { kat. EDDA, Benzol, } 60{ }^{\circ} \mathrm{C}, 16 \text { h, Ultraschall }\end{array}$ & Zersetzung \\
\hline
\end{tabular}




\begin{tabular}{|c|c|c|c|}
\hline \multirow[t]{2}{*}{15} & Boc & 1.2 Äq. Meldrum-Säure, 1.5 Äq. 122 & Multispot-Reaktion \\
\hline & & kat. EDDA, Benzol, $60^{\circ} \mathrm{C}, 5 \mathrm{~h}$, Ultraschall & \\
\hline \multirow[t]{2}{*}{16} & Boc & 1.2 Äq. Meldrum-Säure, 1.5 Äq. 122 & Multispot-Reaktion \\
\hline & & kat. EDDA, Benzol, $80^{\circ} \mathrm{C}, 4 \mathrm{~h}$, Ultraschall & \\
\hline \multirow[t]{2}{*}{17} & Boc & 1.2 Äq. Meldrum-Säure, 3.0 Äq. 122 & Multispot-Reaktion \\
\hline & & kat. EDDA, Benzol, $80^{\circ} \mathrm{C}, 4 \mathrm{~h}$, Ultraschall & \\
\hline
\end{tabular}

Tabelle 5: Versuche zur Optimierung der Domino-Knoevenagel-hetero-Diels-Alder-Reaktionssequenz zur Darstellung von 120/178.

Es wurde auch versucht, das Reaktionsgemisch ohne Isolierung des Cycloaddukts $\mathbf{1 2 0}$ bzw. 178 direkt in der biomimetischen Cyclisierung umzusetzen. Dazu wurde die Reaktionslösung zunächst mit Kaliumcarbonat und Methanol versetzt, um eine Lactonöffnung zu bewirken und dann einer Hydrierung mit Palladium auf Aktivkohle unterzogen. Die gewünschten Produkte 191 und 192 konnten jedoch dabei nur massenspektroskopisch nachgewiesen werden.
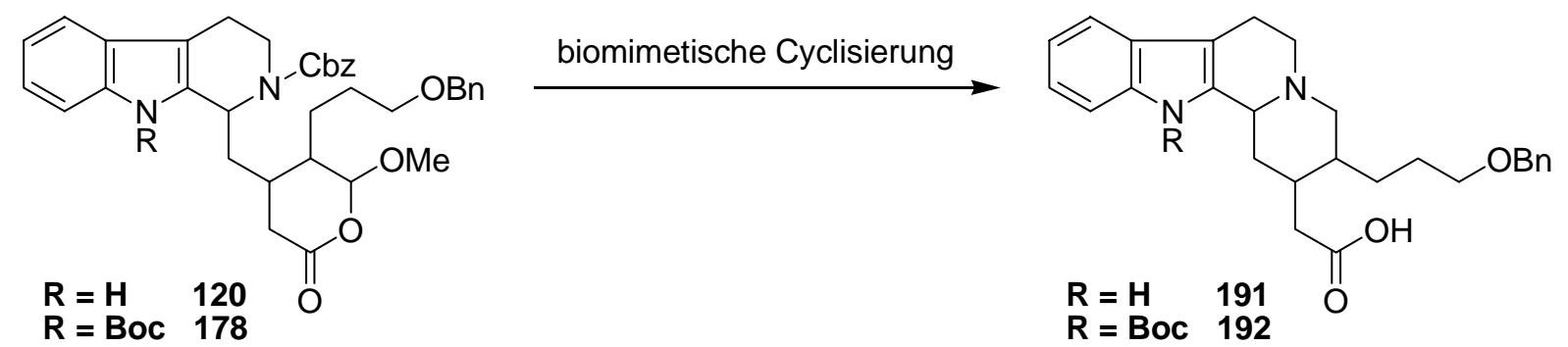

Abbildung 65: Biomimetische Cyclisierung des Domino-Produkte 120 und 178.

In einer alternativen Strategie wurde derweilen versucht, cyclische Enolether als Dienophile einzusetzen. Hierfür wurden die kommerziell erhältlichen Verbindungen Dihydrofuran 140 und Dihydropyran 193 verwendet. In den erhaltenen komplexen Gemischen konnten die gewünschten Domino-Produkte nicht nachgewiesen werden. 


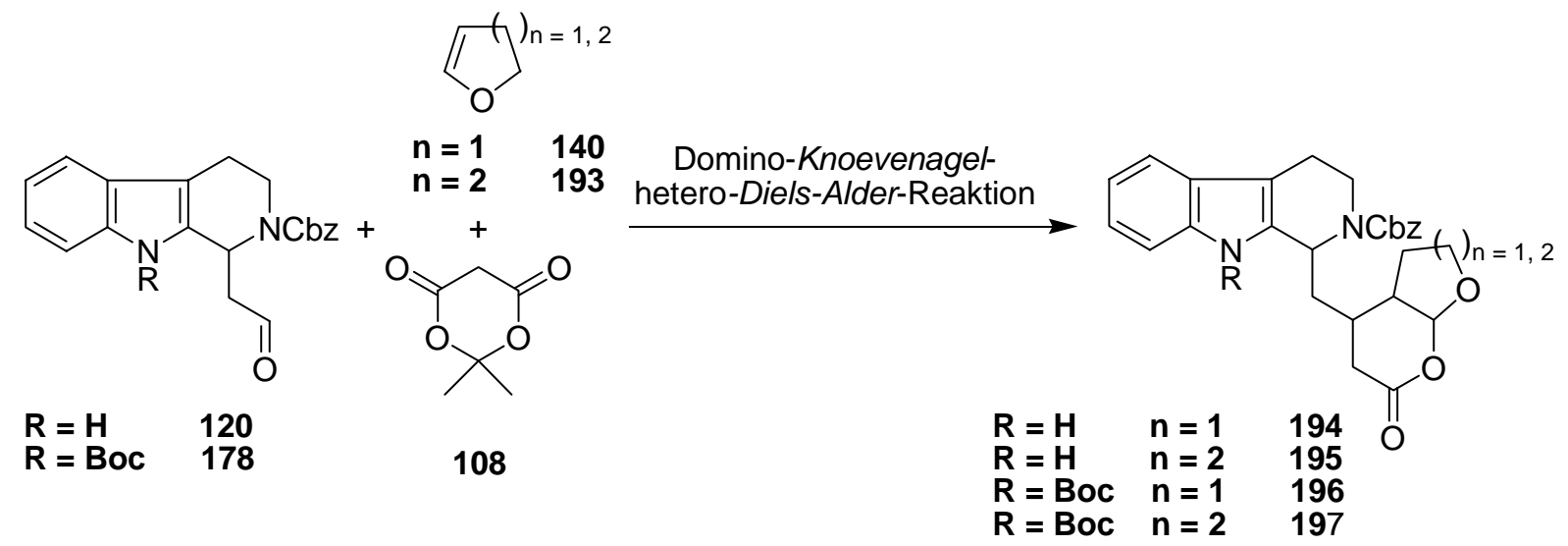

Abbildung 66: Domino Knoevenagel-hetero-Diels-Alder-Reaktion mit kommerziell erhältlichen Dienophilen. 


\section{B) Versuche zur Synthese von Camptothecin}

\section{Versuche zur Synthese des enantiomerenreinen Aldehyds 130}

Im zweiten Teil dieser Arbeit sollte untersucht werden, inwieweit das bei Yohimbin (9) vorgestellte Synthesekonzept auf die Totalsynthese von Camptothecin (44) an-gewandt werden kann Der erfolgreiche Einsatz der Domino-Knoevenagelhetero-Diels-Alder-Reaktion sowie der darauf folgenden biomimetischen Cyclisierung würde so einen völlig neuen synthetischen Zugang zum Aufbau von CamptothecaAlkaloiden erlauben.

Hierfür wurde im ersten Schritt Fumarsäuremonoethylester (198) mit Thionylchlorid zum Säurechlorid aktiviert und nachfolgend mit Anilin (199) und 1.0 Äq. Triethylamin in Dichlormethan innerhalb von $24 \mathrm{~h}$ in das entsprechende Amid 200 überführt. Da diese Reaktion nicht ohne die Bildung mehrerer Nebenprodukte verlief, gestaltete sich die Trennung des Amids vom Reaktionsgemisch als kompliziert und aufwendig. Der Versuch, bei einer Reaktionstemperatur von $0{ }^{\circ} \mathrm{C}$ einen sauberen Reaktionsverlauf zu erzielen, erwies sich leider als erfolglos und bewirkte hinzu eine Herabsetzung der Ausbeute. In der folgenden Michael-Addition mit Propargylamin (201) wurde das Dienophil-Fragment für die angestrebte intramolekulare Diels-Alder-Cyclisierung mit einer mässigen Ausbeute von $57 \%$ eingeführt und anschließend das sekundäre Stickstoff-Atom mit Carbobenzyloxychlorid geschützt. Die Verwendung von Meerwein-Salz sollte nun ermöglichen, ein Lactimether-Intermediat 204 zu bilden, welches dann in einer [4+2]-Cycloaddition das Chinolin 205 liefern sollte. Ein Versuch, diese Umsetzung erfolgreich durchzuführen, scheiterte jedoch. Vermutlich ist dafür die Alterung des Meerwein-Salzes verantwortlich. Bemühungen, diese Reaktion sowie die geplanten weiteren Transformationen zum Aldehyd 130 fortzusetzen, wurden zunächst an dieser Stelle eingestellt. 


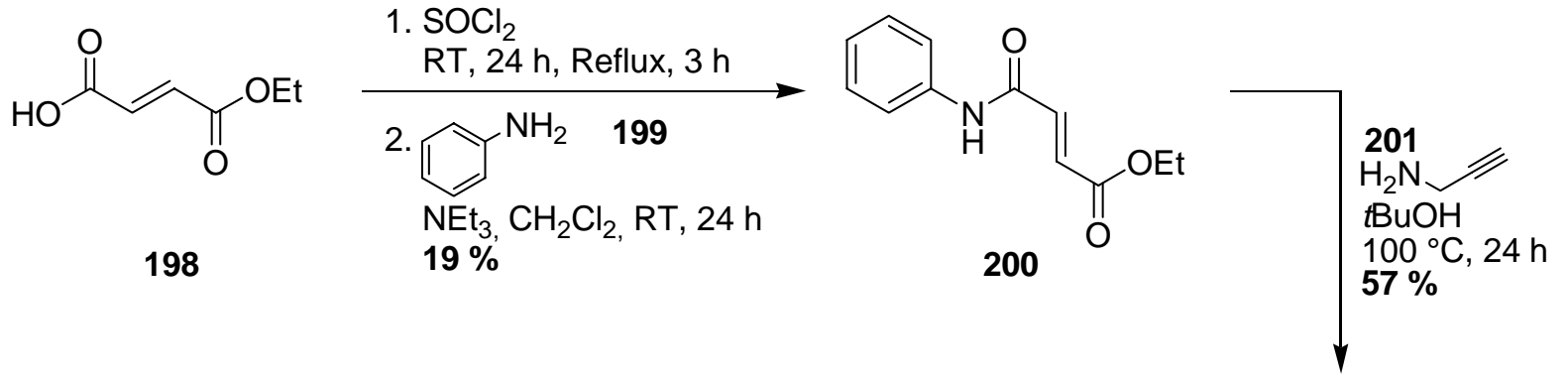

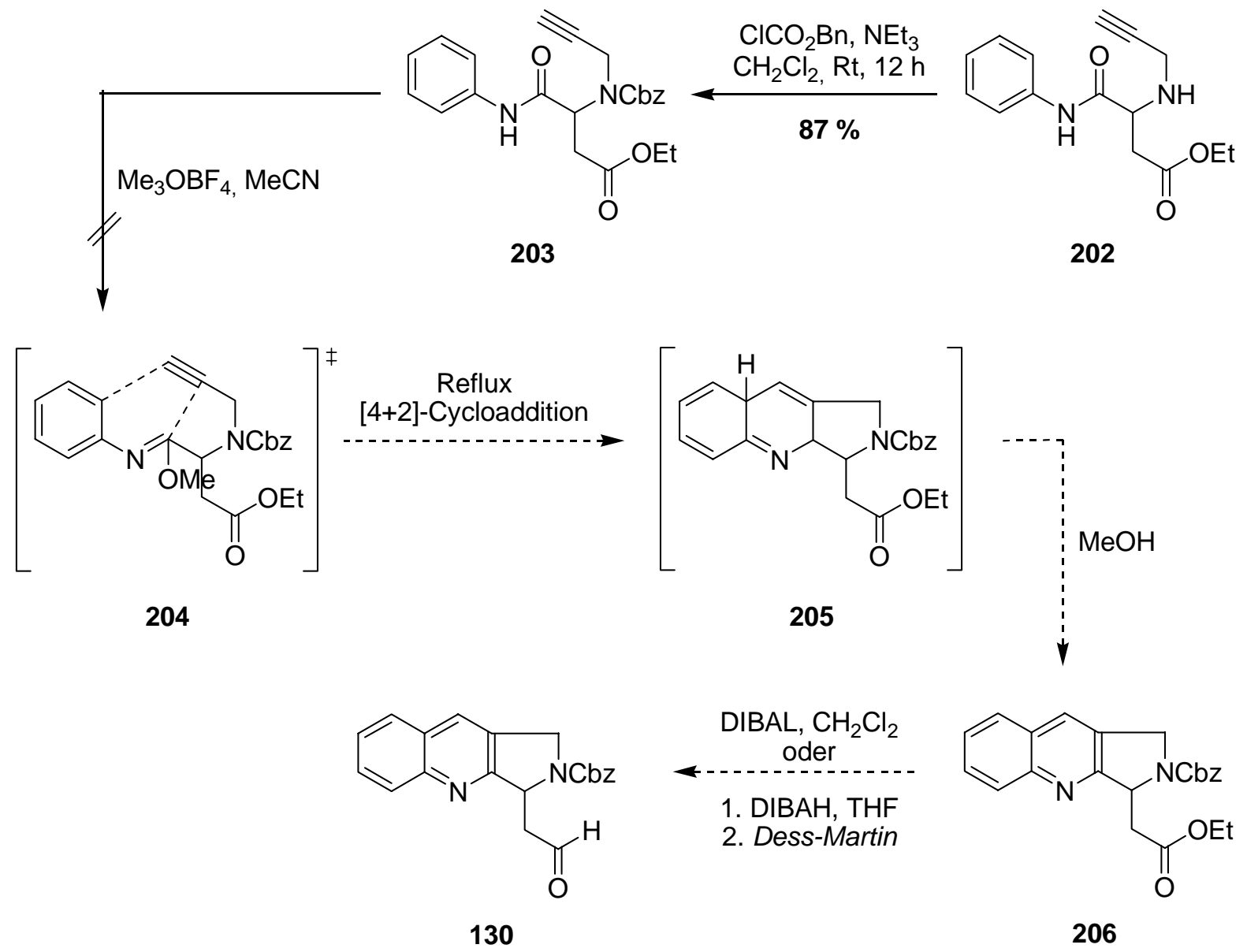

Abbildung 67: Synthesestrategie für den Aldehyd 130.

Parallel zu der oben beschriebenen Synthesestrategie wurde ein alternativer Zugang zu dem Aldehyd 130 untersucht. Dazu wurde die Aminosäure 207 mit Hilfe von Thionylchlorid in Ethanol zum gewünschten Ester 208 in qunatitativer Ausbeute umgesetzt, gefolgt von einer Cbz-Schützung der Amino-Funktionalität. Darauf aufbauend sollte in einer Michael-Additions-, Substitutions- und Decarboxylierungs- 
Sequenz Verbindung 209 dargestellt werden. Allerdings konnte diese Transformation trotz Variation der Reaktionsbedingungen nicht erfolgreich durchgeführt werden.

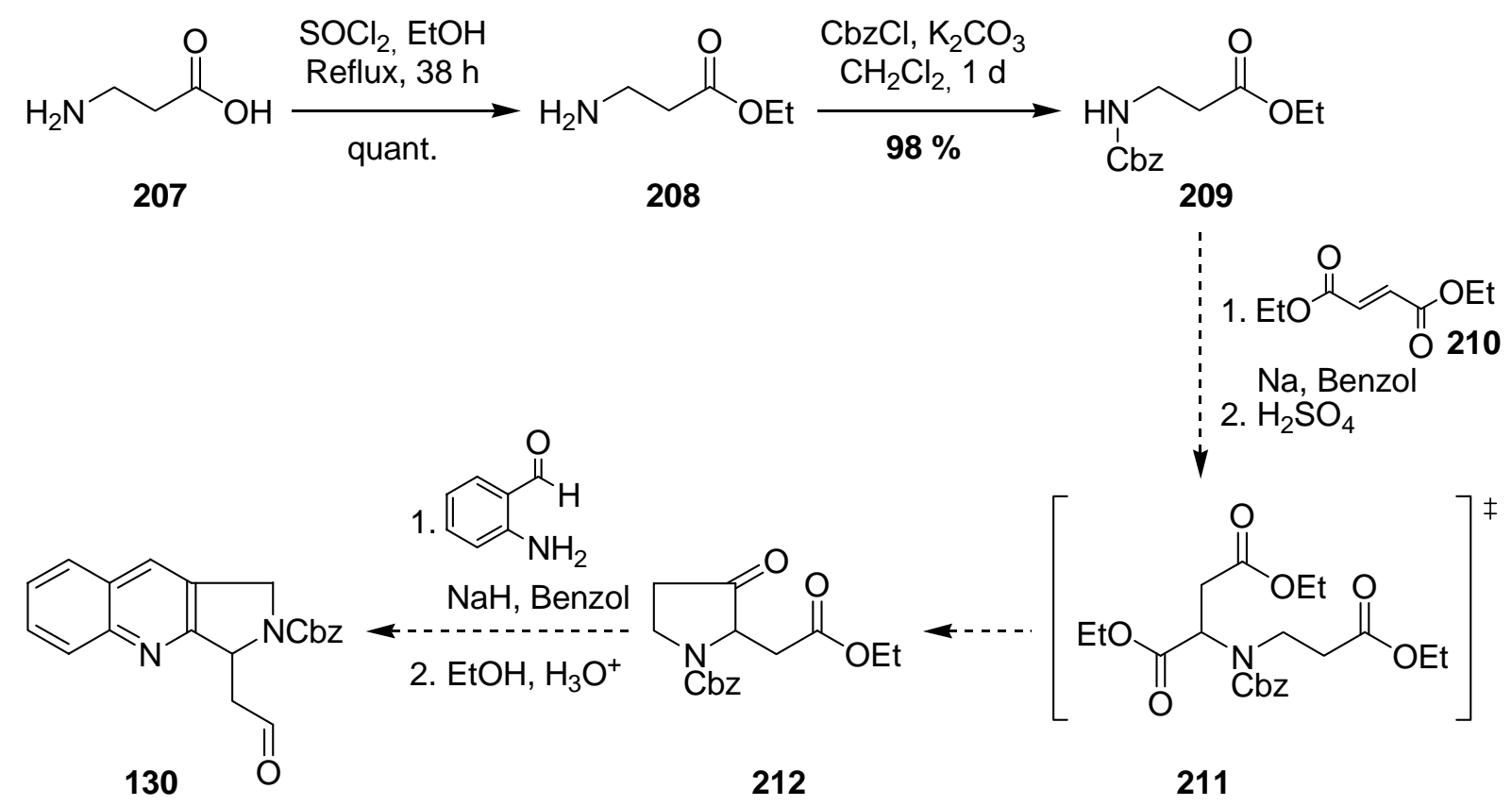

Abbildung 68: Alternative Synthesestrategie für 130.

Aufgrund der Probleme, die bei der Synthese des Aldehyds 130 deutlich wurden, entschloß man sich den Einsatz des herzustellenden Dienophils 129 in der DominoKnoevenagel-hetero-Diels-Alder-Reaktion vorher intensiv an einem Modellsystem zu testen. Entsprechend diesem Vorhaben wurde das Isochinolin-Derivat 218 in einer 5-stufigen Synthese aufgebaut. Begonnen wurde mit einer Bischler-NapieralskiReaktion von 213, wobei der Versuch, Verbindung 214 unter den klassischen Reaktionsbedingungen in guten Ausbeuten zu erhalten, zunächst misslang. Vor allem die Aufarbeitung des schwarzen klebrigen Rückstandes erwies sich als problematisch. Erst in Anlehnung an eine Veröffentlichung der Arbeitsgruppe Bonwell et al. konnte die Ausbeute des Bischler-Napieralski-Produktes 214 erheblich gesteigert werden. Mit einem Reagenzverhältnis von 5 Äq. Trifluormethansulfonsäureanhydrid zu 3 Äq. Dimethylaminopyridin lieferte die intramolekulare Kondensationsreaktion des Amids 213 in Dichlormethan innerhalb von 5 Tagen bei $0{ }^{\circ} \mathrm{C}$ bis Raumtemperatur akzeptable Ausbeuten von $70 \%$. Palladium-katalysierte Hydrierung des vinylogen Urethans 214 
bei 4 bar in einem Ethanol/Essigsäure-Gemisch ergab Verbindung 215 in $73 \%$ Ausbeute. Die anschließende Reduktion mit Lithiumaluminiumhydrid in THF zum entsprechenden Alkohol 216 mit nachfolgender Schützung der Amino-Funktionalität mit Carbobenzyloxychlorid in einem Zweiphasensystem bestehend aus $\mathrm{CH}_{2} \mathrm{Cl}_{2}$ und gesättigter wässriger $\mathrm{NaHCO}_{3}$-Lösung lieferte 217. Für die Oxidation des Alkohols 217 standen verschiedene Reagenzien zur Verfügung. Die Verwendung von 1.5 Äq. Dess-Martin-Periodinan als Oxidationsmittel stellte sich dabei als die geeigneteste Methode heraus und führte so zu der Synthese des Aldehyds 218 in einer Ausbeute von $78 \%$.<smiles>CCOC(=O)CC(=O)NCCc1ccccc1</smiles><smiles>CCOC(=O)/C=C1\NCCc2ccccc21</smiles>

$\mathrm{Tf}_{2} \mathrm{O}$, DMAP

$\mathrm{CH}_{2} \mathrm{Cl}_{2}$

$0{ }^{\circ} \mathrm{C} \rightarrow \mathrm{RT}, 5 \mathrm{~d}$

$70 \%$<smiles>CCOC(=O)/C=C1\NCCc2ccccc21</smiles>

214<smiles>[R15]CC(=O)OC(=O)CC1NCCc2ccccc21</smiles>

215

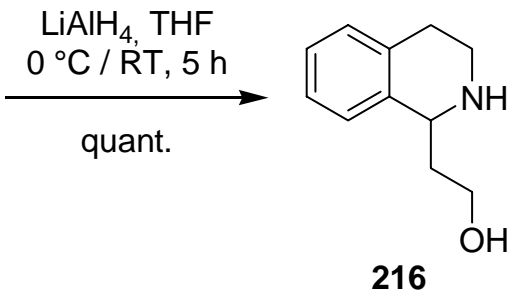

$\mathrm{CbzCl}, \mathrm{CH}_{2} \mathrm{Cl}_{2}$ ges. $\mathrm{NaHCO}_{3}$ $\Delta, 3.5 \mathrm{~h}$ $70 \%$<smiles>O=CCC1c2ccccc2CCN1C(=O)[O-]</smiles>

218
Dess-Martin Periodinan

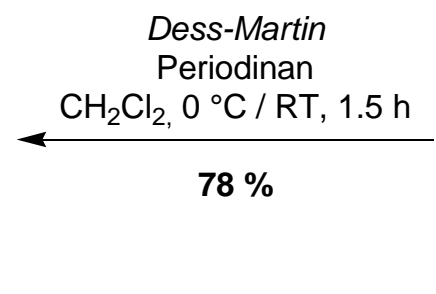

$78 \%$<smiles>O=C([O-])N1CCc2ccccc2C1CCO</smiles>

Abbildung 69: Modellsystem 218 für die Domino-Knoevenagel-hetero.Diels-Alder-Reaktion. 


\section{Versuche zur Synthese des Silylenolethers 129}

Für die Synthese des Silylenolethers 129 bot sich die Ketocarbonsäure 131 als käufliche Ausgangsverbindung an. Eine fünfstufige Synthesesequenz sollte es gestatten, das Dienophil 129 erfolgreich aufzubauen. Zuerst wurde $131 \mathrm{mit}$ katalytischen Mengen an Trimethylsilylchlorid und 2,2-Dimethoxypropan in Methanol bei Raumtemperatur verestert. Überraschender Weise gelang es aber nicht die KetoFunktionalität zum korrespondierenden Alkohol 220 im darauffolgenden Schritt zu reduzieren (Abbildung 70).<smiles>CCC(=O)C(=O)O</smiles>

131

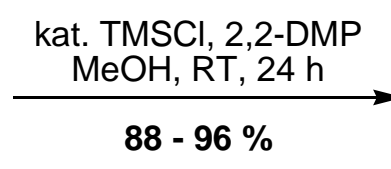

$88-96 \%$<smiles>CCC(=O)C(=O)OC</smiles>

219

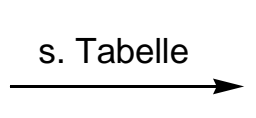<smiles>CCC(O)C(=O)OC</smiles>

220

Abbildung 70: Versuche zur Synthese des Hydroxyesters 220.

Die Reduktion eines Ketons zum Alkohol in Gegenwart einer Carbonsäure wird üblicherweise mit Natriumborhydrid erreicht. In diesem Fall konnte jedoch kein Reduktionsprodukt 220 isoliert werden. Erhöhung der Natriumborhydrid-Menge um 50 \% (Nr. 2) auf 15.0 Äquivalente ergab eine klare Flüssigkeit, deren Molekülpeak in der massenspektroskopischen Messung der gewünschten Verbindung 220 entspricht, allerdings stimmen die NMR-Spektren nicht mit den für 220 erwarteten Daten überein. Eine weitere Variation der Lösungsmittelsysteme, der Temperatur, Reaktionszeit und der Äquivalente an Reduktionsmittel führten ebenfalls nicht zum Erfolg. Der Versuch, eventuell gebildeten Alkohol 220 ohne vorherige Isolierung als TBS-geschützte Verbindung 221 abzufangen, ließ sich ebenso wenig realisieren. Als weitere Möglickeit bot sich eine Hydrierung mit Palladium auf Aktivkohle an, die aber wiederum eine Polymerisation zur Folge hatte (Tabelle 6). 


\begin{tabular}{|c|c|c|}
\hline Nr. & Reaktionsbedinungen & Ergebnisse \\
\hline 1 & 10 Äq. $\mathrm{NaBH}_{4}, \mathrm{MeOH}, \mathrm{RT}, 30 \mathrm{~min}$ & kein Produkt \\
\hline 2 & 15 Äq. $\mathrm{NaBH}_{4}, \mathrm{MeOH}, \mathrm{RT}, 30 \mathrm{~min}$ & $\begin{array}{l}\text { nur massenspektro- } \\
\text { skopischer Nachweis }\end{array}$ \\
\hline 3 & 0.25 Äq. $\mathrm{NaBH}_{4}, i \mathrm{PrOH}, \mathrm{RT}, 3 \mathrm{~h}$ & kein Produkt \\
\hline 4 & $\begin{array}{l}\text { 1. } 1.4 \text { Äq. } \mathrm{NaBH}_{4}, \mathrm{CH}_{2} \mathrm{Cl}_{2}, \mathrm{MeOH}, \mathrm{RT}, 30 \mathrm{~min} \\
\text { 2. } 1.18 \text { Äq. TBSCl, Imidazol, DMF, RT, } 12 \mathrm{~h}\end{array}$ & kein Produkt \\
\hline 5 & 3.0 Äq. $\mathrm{NaBH}_{4}, \mathrm{THF}, \mathrm{MeOH},-78^{\circ} \mathrm{C}, 30 \mathrm{~min}$ & Zersetzung \\
\hline 6 & 6.5 Äq. $\mathrm{NaBH}_{4}, \mathrm{MeOH}, \mathrm{RT}, 30 \mathrm{~min}$ & Polymerisierung \\
\hline 7 & $\mathrm{H}_{2}, \mathrm{Pd} / \mathrm{C}, \mathrm{MeOH}, \mathrm{RT}, 20 \mathrm{~h}$ & Polymerisierung \\
\hline
\end{tabular}

Tabelle 6: Versuche zur Durchführbarkeit der Reduktion von 219.

Alternativ sollte für die Synthese der Hydroxycarbonsäure 219 ein anderer Weg beschritten werden. Dazu wurde die $\alpha$-Aminosäure 222 mit Hilfe von Natriumnitrit in Essigsäure acyliert und anschließend in der nächsten Transformation der Versuch unternommen, die Acetyl-Gruppe mittels Thionylchlorid zu entfernen (Abbildung 71). Die wiederholten Versuche des zweiten Schrittes führten bei jedem Experiment zur Gewinnung eines grünen Feststoffes, dessen Struktur sich nicht ermitteln ließ. Der erhoffte Alkohol 219 wurde dabei nicht gefunden.<smiles>CCC(N)C(=O)O</smiles>

222

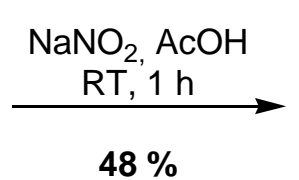

$48 \%$<smiles>CCC(OC(C)=O)C(=O)O</smiles>

223<smiles>CCC(O)C(=O)OC</smiles>

219

Abbildung 71: Alternative Synthese von 219.

Die Öffnung eines Epoxides über eine nukleophile Substitutionsreaktion bot eine dritte Möglichkeit zur Darstellung des Alkohols 219. Hierzu sollte die Doppelbindung des Esters 224 zuerst mit para-Chlorperbenzoesäure epoxidiert werden (Abbildung 72). 
Auch wenn das Epoxid 225 nicht gewonnen werden konnte, ist davon auszugehen, dass diese Reaktion grundsätzlich funktionieren solle. Womöglich wurde bei der Aufarbeitung der Reaktionslösung eine Epoxid-Öffnung bewirkt, so dass noch Optimierungsarbeit diesbezüglich erforderlich ist.<smiles>C/C=C/C(=O)OC</smiles>

224

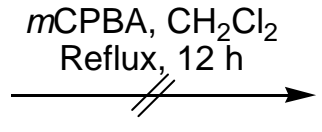

Tre

225

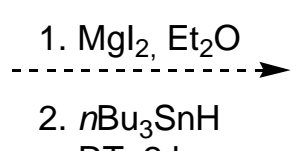

$\mathrm{RT}, 2 \mathrm{~h}$<smiles>CCC(O)C(=O)OC</smiles>

219

Abbildung 72: Alternative Synthese von 219.

Ausgehend von der Hydroxycarbonsäure 219 fehlen zur Vollständigkeit dieser Synthese des Dienophils 129 nur eine Schützung des Alkohols 219 mit TBSCl, die Erzeugung des entsprechenden Enolats mit einer Base und dessen Silylierung zu 129 im abschließenden Schritt (Abbildung 73).<smiles>CCC(O)C(=O)OC</smiles>

219

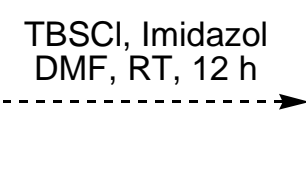<smiles>CCC(O[Sb](=O)(=O)c1ccccc1)C(=O)OC</smiles>

221

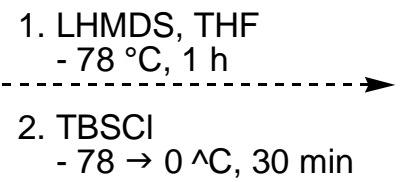

- $78 \rightarrow 0^{\wedge} \mathrm{C}, 30 \mathrm{~min}$<smiles>CC/C([OH2+])=C(/[OH2+])OC</smiles>

129

Abbildung 73: Synthesestrategie für die Darstellung des Silylenolethers 129. 


\section{Zusammenfassung}

Die biologische Wirksamkeit vieler Naturstoffe haben die Menschen bereits seit ältesten Zeiten für sich zu nutzen gewusst. Insbesondere in der Naturstoffklasse der Alkaloide findet man eine Vielzahl an Verbindungen, die aufgrund ihrer naturbedingten Aktivität unter anderem als Pharmaka zur Behandlung von Krankheiten eingesetzt werden. Deren Darstellung stellt noch heute eine besondere Herausforderung an den Synthesechemiker, gilt es doch stereochemische und regioselektive Kriterien zu beachten, um komplizierte Strukturen gezielt zu verändern oder gänzlich zu synthetisieren. Da die Isolierung grösserer Mengen aus natürlichen Quellen wie Mikroorganismen, Schwämmen, Pflanzen oder Metazoen aufgrund deren begrenzten Verfügbarkeit nur unzureichend gedeckt wird, ist eine chemische Totalsynthese oftmals unerlässlich. Darüber hinaus erlaubt dies Modifizierungen dieser Verbindungen um Struktur-Wirkung-Beziehungen zu erarbeiten, die Aktivität zu steigern, Nebenwirkungen zu minimieren, die pharmakologischen und chemischen Eigenschaften zu verändern und um aktive Teilstrukturen zu erkennen.

In Hinblick auf Effizienz, Wirtschaftlichkeit und Ressourcenschonung, die bei modernen Syntheseverfahren im zunehmenden Masse in den Mittelpnkt rücken, erweisen sich Domino-Reaktionen als besonders wertvoll. Hierzu zählt auch die im Arbeitskreis von L.F. Tietze entwickelte Domino-Knoevenagel-hetero-Diels-AlderReaktion mit einer darauf folgenden zweiten Domino-Sequenz, bestehend aus einer Solvolyse, Hydrogenolyse, Kondensation und Hydrierung, mit deren Hilfe ein gewählter Zugang zu monoterpenoiden Indolalkaloiden der Vallesiachotamin- und Corynanthe-Gruppe als auch der Isochinolinalkaloiden vom Ipecacuanha-Typs gelingt. Auf diese Weise konnten unter anderem Ementin (110), Hirsutin, Dihydrocorynanthein, Dihydroantirhin und Tubulosin synthetisch zugänglich gemacht werden (Abbildung 74). 
<smiles>CC(=O)[C@@H]1[C@H](O)CC[C@@H]2CN3CCc4c([nH]c5ccccc45)[C@H]3C[C@H]21</smiles>

(+)-Yohimbin 9<smiles>CC[C@@]1(O)C(=O)OCc2c1cc1n(c2=O)Cc2cc3ccccc3nc2-1</smiles>

(+)-Camptothecin 44<smiles>CCCCCCN1CCc2cc(OC)c(OC)cc2[C@H]1C[C@H]1C[C@@H]2c3cc(OC)c(OC)cc3CCN2C[C@H]1CC</smiles>

Abbildung 74: Alkaloide.

Ziel dieser Dissertaion war es, mit Hilfe einer drei Komponenten DominoKnoevenagel-hetero-Diels-Alder-Reaktion und mit dem Einsatz der am Beispiel der Totalsynthese von Emetin (110) vorgestellten biomimetischen Cyclisierung das Yohimban-Alkaloid Yohimbin (9) und das Camptotheca-Alkaloid Camptothecin (44) enantiomerenrein aufzubauen. Zuvor sollten die stereogenen Zentren, die nicht über die Domino-Sequenz aufgebaut werden können, in Katalysator-kontrollierten Reaktionen synthetisiert werden. Die asymmetrische Transferhydrierung nach Noyori erschien hierfür als geeignet. Als effizienteste Methode zur Synthese des 1,2,3,4Tetrahydro- $\beta$-carbolin-Systems 123 hatte sich die Pictet-Sprengler-Reaktion, die bereits mehrfach in Arbeitskreis von L. F. Tietze durchgeführt worden war, etabliert. Erste Versuche, Tryptamin-Hydrochlorid (17) mit der $\alpha$-Ketocarbonsäure 126 in Ethanol zum korrespondierenden Ester 123 umzusetzen, ergaben in Übereinstimmung mit vorherigen Ergebnissen anderer Arbeiten zunächst Ausbeuten von nur 55 \%. Nach Optimierung der Reaktionsbedingungen konnte die Aubeute von 123 auf $80 \%$ gesteigert werden. Die Synthese des Imins 124 für die geplante asymmetrische Transferhydrierung wurde über eine einfache Oxidation des racemischen Tetrahydro$\beta$-carbolin-Derivates 123 mit $\mathrm{KMnO}_{4}$ bei $0{ }^{\circ} \mathrm{C}$ erreicht. Die Aubeuten variierten dabei in Abhängigkeit der eingesetzten Menge am Edukt 123. Das beste Ergebnis konnte in einem Ansatz mit $10 \mathrm{~g}$ des Esters mit einer Ausbeute von $86 \%$ erzielt werden (Abbildung 75). 


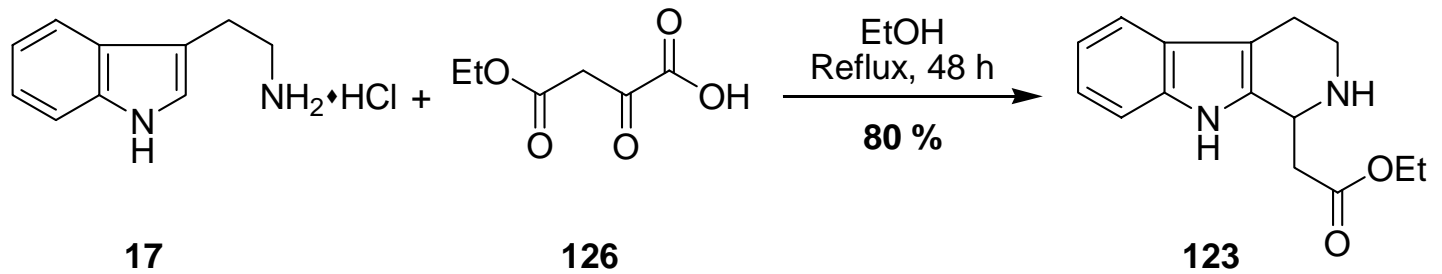<smiles>CCOC(=O)CC1=NCCc2c1[nH]c1ccccc21</smiles>

Abbildung 75: Synthese des Imins 124 für die asymmetrische Transferhydrierung.

Für die anschließende asymmetrische Transferhydrierung standen die beiden chiralen Ruthenium-Katalysatoren 125 und 159 zur Auswahl, wobei sich insbesondere 125 als effizienter Katalysator für die Reduktion von Iminen mit einem Tetrahydro- $\beta$ carbolin-Grundgerüst erwiesen hatte. 159 hatte sich dagegen bei der Synthese enantiomerenreiner Chinolin-Derivate bewährt.
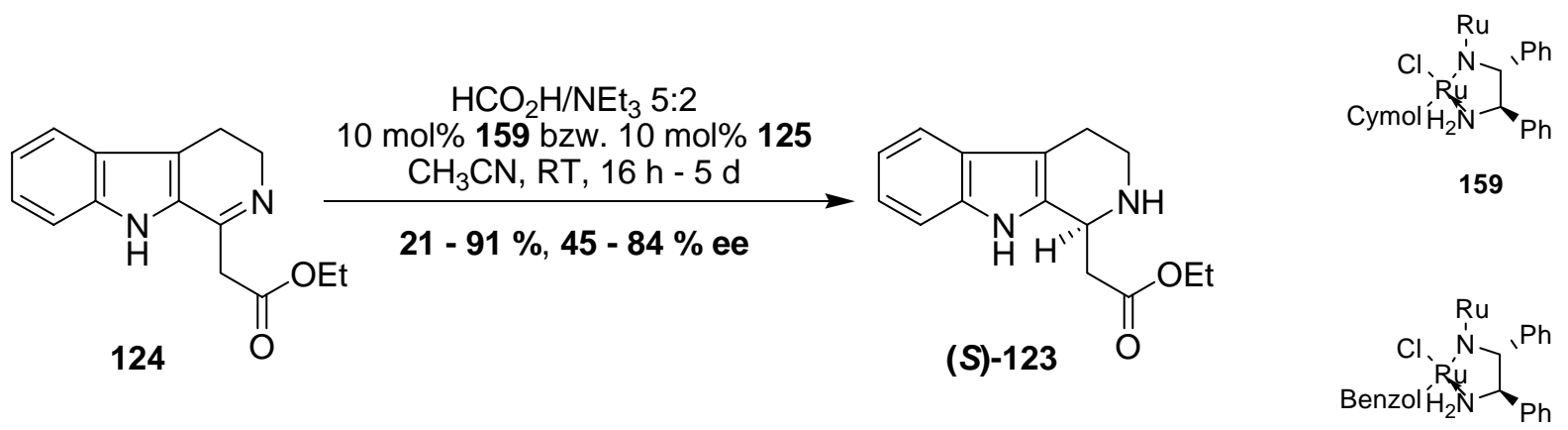

125

Abbildung 76: Asymmetrische Transferhydrierung.

Trotz umfangreicher Versuche, durch Variation der Katalysatormenge, des Lösungmittels sowie der Reaktionszeit konnten aber nur moderate bis gute Ausbeuten 
an (S)-123 erhalten werden. Ebensowenig gelang es, gute ee-Werte zu reproduzieren. Stattdessen bewegten sich die Enantiomerenüberschüsse zwischen $46-84 \%$ ee (Abbildung 76).

Daraufhin wurde getestet, ob mittels eines Umweges, der auf der Verwendung der geschützten Alkohole 167 und 168 basierte, die Einführung des stereogenen Zentrums mittels der Noyori-Reduktion gelingen könnte. Für diese Synthesestrategie wurde die Ausgangsverbindung 123 zunächst mit Lithiumaluminiumhydrid zum korrespondierenden Alkohol 164 in hohen Ausbeuten von 88\% reduziert und dieser anschließend als TiPS- bzw. TBS-Ether geschützt (Abbildung 77). Die Oxidation mit Kaliumpermanganat lieferte die gewünschten Substrate 167 und 168 für die asymmetrische Hydrierung, die allerdings leider zur keiner Verbesserung führte.<smiles>O=C([O-])CC1NCCc2c1[nH]c1ccccc21</smiles>
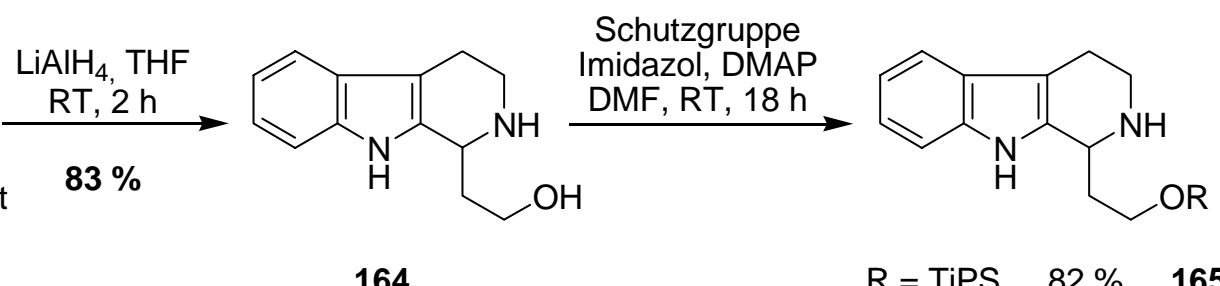

164
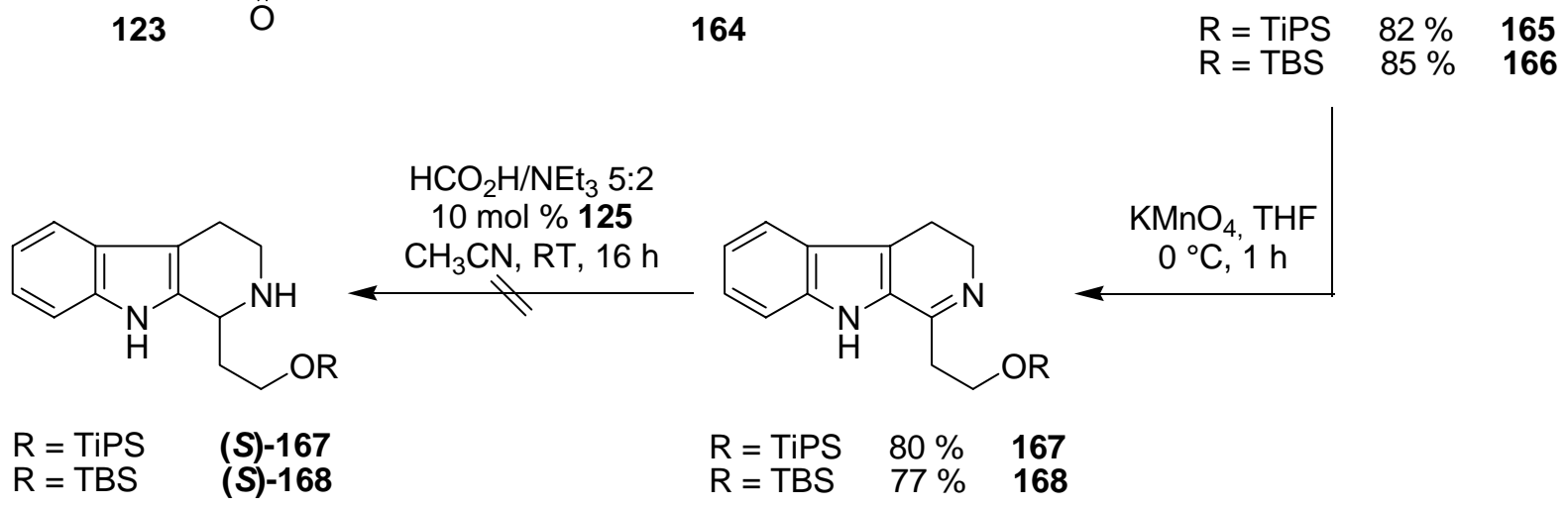

Abbildung 77: Versuche zur Synthese der enantiomerenreiner Aldehyde (1S)-165 und (1S)-168.

Basierend auf diesen Ergebnissen wurde die Durchführbarkeit der Noyori- Hydrierung eingehend an einem literaturbekannten Modellsystem untersucht.

Hierzu wurde zunächst das Phenylethylamin 169 zu 86 \% Ausbeute zu 170 acyliert. Eine Bischler-Napieralski-Isochinolin-Synthese lieferte das gewünschte Imin 171 
(Abbildung 78), welches dann unter bekannten Reaktionsbedingungen mit $0.2 \mathrm{~mol} \%$ des Katalysators 159 umgesetzt wurde. Hierbei konnte allerdings nur das Edukt 171 reisoliert werden. Eine Erhöhung der Katalysatormenge auf $10 \mathrm{~mol} \%$ führte zwar zu der gewünschten Zielverbindung 172 in sehr guten Ausbeuten von $80 \%$, allerdings nur mit einem ee-Wert von $13 \%$.

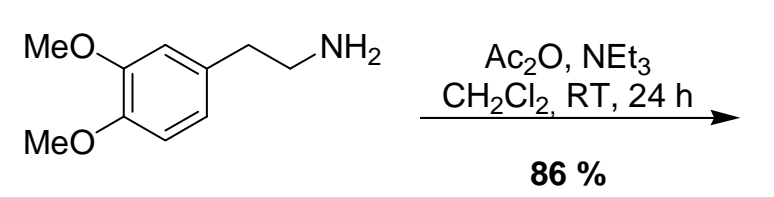

169<smiles>COc1ccc(CCNC(=O)c2ccccc2)cc1OC</smiles>

170

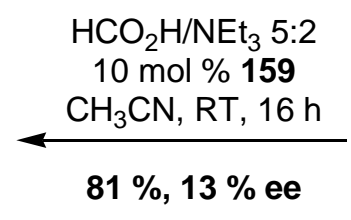

$81 \%, 13 \%$ ee

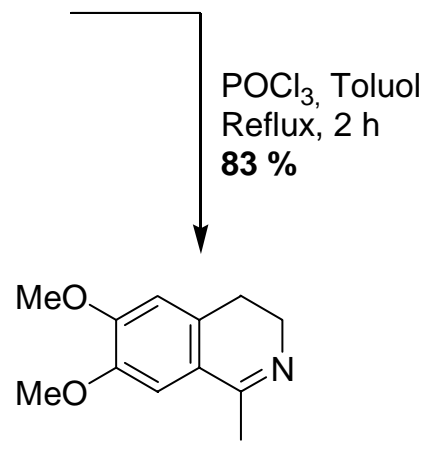

171

Abbildung 78: Asymmetrische Transferhydrierung an einem Modellsystem.

Aufgrund dieser negativen Resultate wurde daraufhin die enantioselektive Reaktionsführung zunächst aufgegeben und der Aldehyd 121 in racemischer Form aufgebaut.

Hierbei wurde Tryptamin-Hydrochlorid (17) mit der Ketosäure 126 unter den zuvor optimierten Bedingungen zum Carbolin 123 umgesetzt. Die nachfolgende selektive Schützung des sekundären Stickstoff-Atoms mit $\mathrm{CbzCl}$ gefolgt von Reduktion des Esters 173 mit Diisobutylaluminiumhydrid führte zum gewünschten Produkt 121 und somit zu dem ersten Baustein für die geplante Domino-Knoevenagel-hetero-DielsAlder-Reaktion (Abbildung 79). 

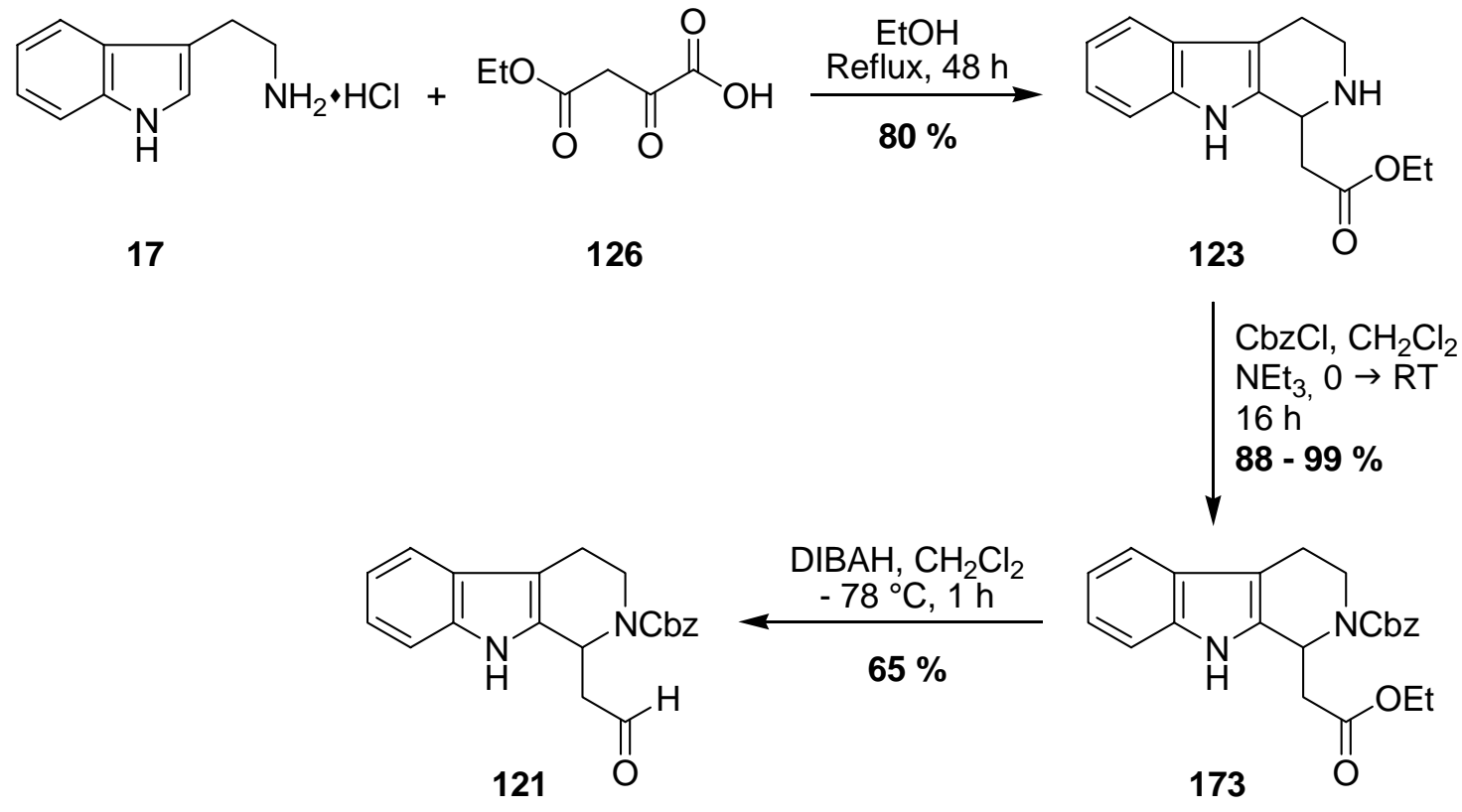

Abbildung 79: Synthese des racemischen Aldehyds 121.

Zusätzlich und in Hinblick auf eine stereoselektive Reaktionsführung bei dem Domino-Knoevenagel-hetero-Diels-Alder-Prozess sollte der Carbolin-Aldehyd $\mathbf{1 7 3}$ in einer NBoc-geschützten Form synthetisiert werden. Dafür wurde der Ester 173 zuerst einer Boc-Schützung und dann einer DIBAH-Reduktion zum entsprechenden Aldehyd 118 unterzogen (Abbildung 80).<smiles>CCOC(=O)CC1c2[nH]c3ccccc3c2CCN1C(=O)OCc1ccccc1</smiles>

173

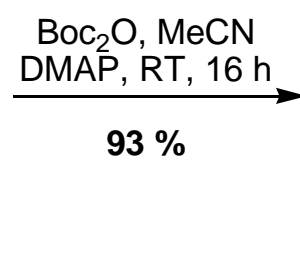<smiles>[R4]CC1c2[nH]c3ccccc3c2CCN1C(=O)OCc1ccccc1</smiles>

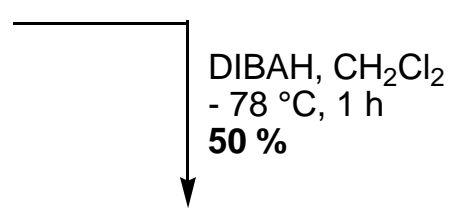

118<smiles>O=CCC1c2[nH]c3ccccc3c2CCN1C(=O)O</smiles>

Abbildung 80: Synthese von dem Aldehyd 118. 
Im nächsten Schritt sollte der Enolether 135 als zweiter Baustein für die DominoKnoevenagel-hetero-Diels-Alder-Reaktion über eine säurekatalysierte Eliminierung eines Alkohols aus den entsprechenden Acetalen generiert werden. Ausgehend vom kommerziell erhältlichen 1,5-Pentandiol (127) wurde eine Alkohol-Funktionalität mit TBSCl in Gegenwart von Imidazol in DMF mit einer sehr guten Ausbeute von $92 \%$ geschützt. Der monogeschützte Alkohol 132 wurde anschließend durch eine Oxidation mit $\mathrm{NaClO}$ und katalytischen Mengen an TEMPO in den Aldehyd 133 überführt und dieser wiederum im letzten Schritt in den gewünschten Acetal 134 umgewandelt (Abbildung 81). Eine Eliminierung von Methanol aus 134 mit Kaliumhydrogensulfat ist in der nächsten Transformation nicht beobachtet worden. Weitere Versuche, durch Variation der Säure, die eigentlich als Katalysator die Spaltung der Alkoholkomponente veranlasst, den Enolether $\mathbf{1 3 5}$ herzustellen, blieben ebenfalls ergebnislos. Weder $p$-Toluolsulfonsäure noch Diphenylphosphinsäure führten zum Erfolg.

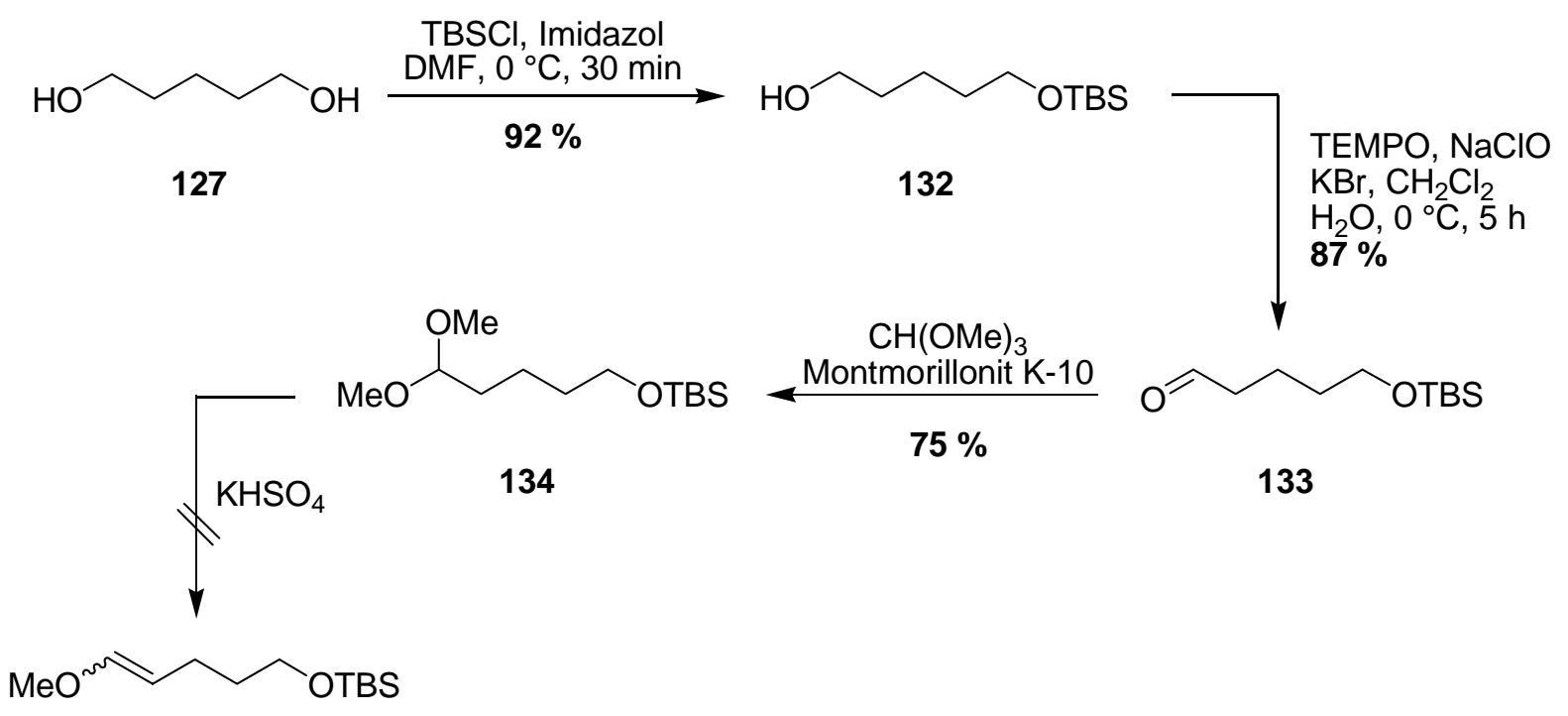
135

Abbildung 81: Versuche zur Synthese des Enolethers 135 mittels einer Säure-katalysierten Eliminierung.

Die erfolglosen Versuche zur Synthese von Verbindung 135 legten die Überlegung nahe, anstelle von 134 ein Acetal mit veränderter Alkoholschutzgruppe der Eliminierungsreaktion zum entsprechenden Enolether auszusetzen. Die Wahl fiel auf das 
benzylgeschützte Derivat 139 (Abbildung 82). Analog zur Synthesestrategie von 134 konnte so das Acetal 139 in $28 \%$ Ausbeute über vier Stufen erhalten werden. Leider erwies sich aber auch hier die Eliminierungsreaktion mit Kaliumhydrogen-sulfat als nicht durchführbar.

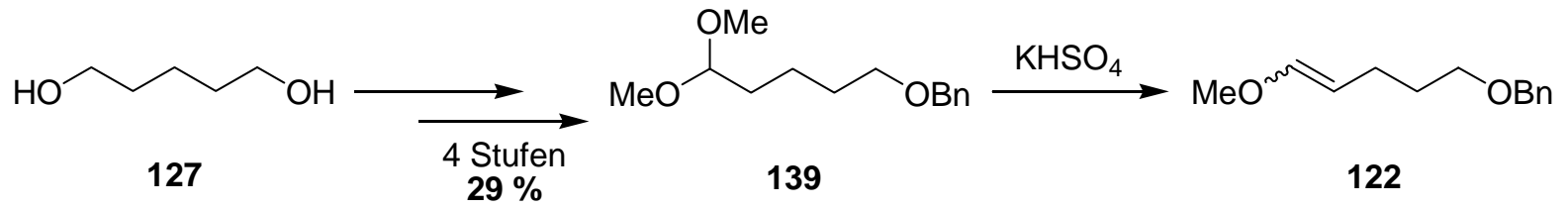

Abbildung 82: Versuche zur Synthese des Enolethers 122.

Die ergebnislosen Bemühungen, die Enolether 136 und 122 über eine säurekatalysierte Eliminierung von einem Alkohol aus einem Aldehydacetal zugänglich zu machen, machten eine neue Synthesestrategie unumgänglich. In einem alternativen Konzept wurde daher versucht, über eine Wittig-Reaktion zu einem Enolether vom Typ 122 zu gelangen. Dazu wurde $\gamma$-Butyrolacton (144) mit Diisobutylaluminiumhydrid zum Lactol 141 in $71 \%$ Ausbeute reduziert, das sich im Gleichgewicht zu seinerm offenkettigen Aldehyd 142 befindet. Wittig-Reaktion mit (Methoxymethyl)triphenylphosphoniumchlorid führte zum Enolether 146 in guten Ausbeuten, dessen Schützung mit Benzylbromid die Synthese des Dienophils vervollständigte (Abbildung 83).

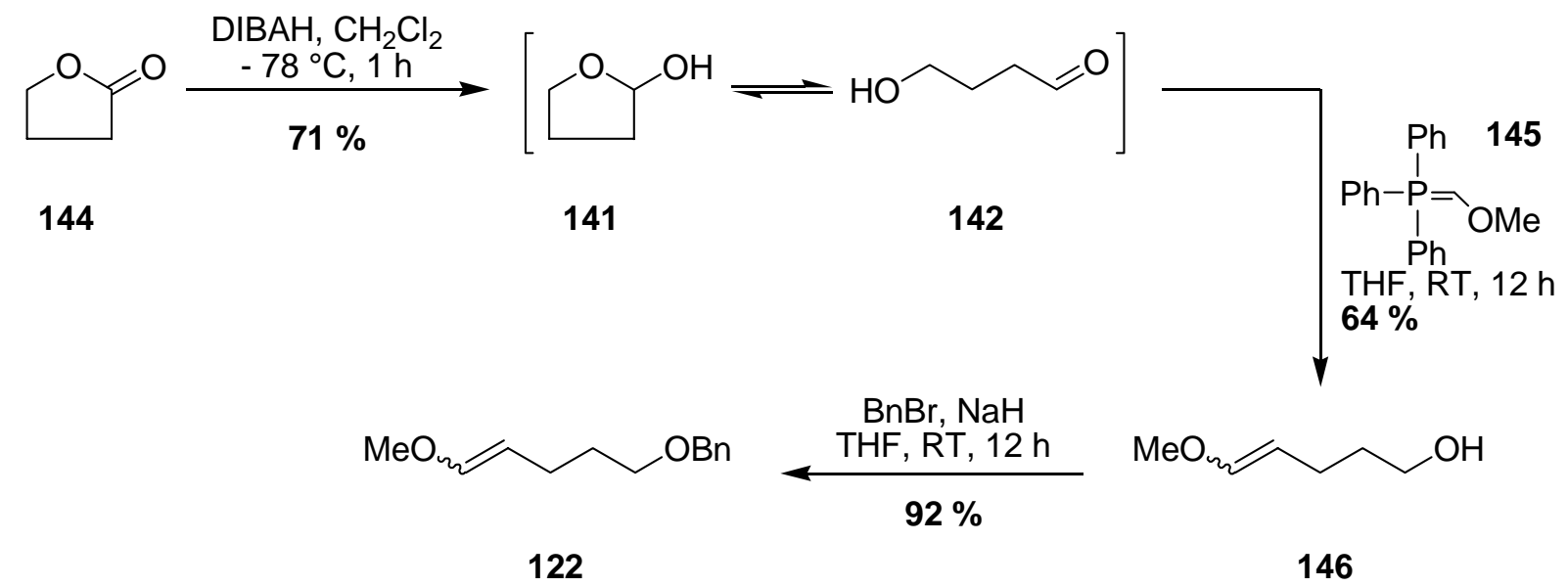

Abbildung 83: Synthese des Enolethers 122. 
Zusätzlich wurden auch Versuche unternommen, Derivate dieser Verbindung herzustellen, andere Enolether aufzubauen, die jedoch nicht erfolgreich waren.

Die Domino-Knoevenagel-hetero-Diels-Alder-Reaktion mit dem racemischen Aldehyd 121 sowie mit dem Boc-geschützten Amin 118, Meldrum-Säure (108) und dem Enolether 122 konnte trotz intensiver Versuche zur Optimierung dieses Prozesses nur in einem Fall (Abbildung 84) das Domino-Produkt 178 liefern. Dabei konnten lediglich $12 \%$ von 178 isoliert werden. Wiederholung der Transformation resultierte entweder in komplexen Gemischen, die sich nicht voneinander trennen ließen oder führte zur Zersetzng der Lactone 120 und 178. Daher wurde 178 sofort in der darauffolgenden biomimetischen Cyclisierung ohne vorherige Reinigung eingesetzt. Das gebildete Substrat 192 lag dabei in so geringer Menge in der Reaktionslösung vor, dass es nur massenspektrometrisch nachgewiesen werden konnte.

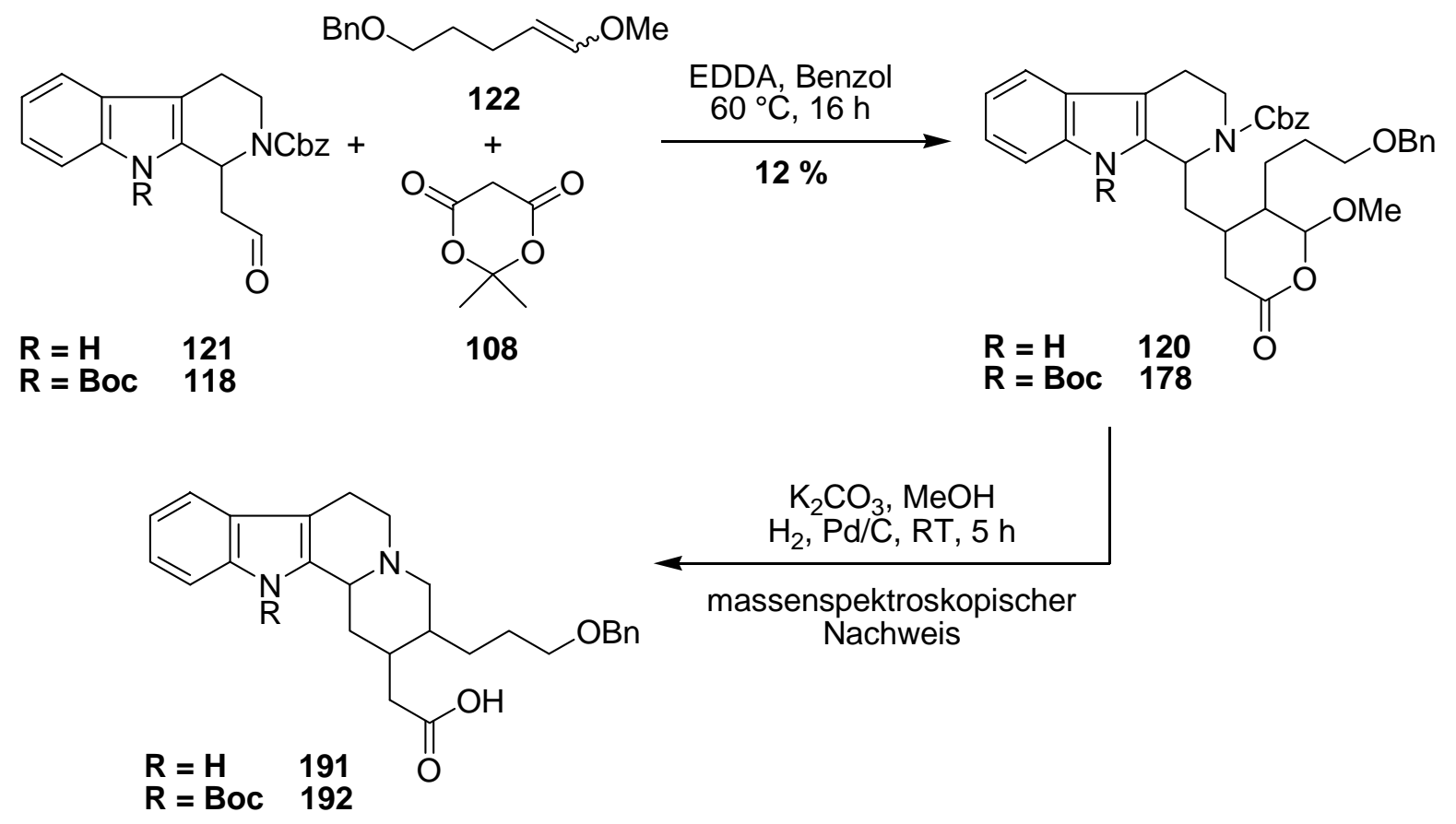

Abbildung 84: $\quad$ Domino-Knoevenagel-hetero-Diels-Alder-Reaktion.

Der Einsatz von cyclischen Ethern wie den kommerziell erhältlichen Verbindungen Dihydrofuran 140 und Dihydropyran 193 als Dienophile ergab gleichfalls komplexe 
Gemische, in denen die gewünschten Domino-Produkte mittels Massenspektrometrie nicht detektiert werden konnten.

Für die Totalsynthese von Campthotecin (44) war analog der Synthesestrategie von Yohimbin (9) geplant, das Corynanthe-Grundgerüst mit Hilfe der bereits vorgestellten Domino-Sequenz und anschliessender biomimetischer Cyclisierung aufzubauen. Der für den Domino-Knoevenagel-hetero-Diels-Alder-Prozess benötigte Aldehyd 130 sollte über zwei unterschiedliche Wege zugänglich gemacht werden. Das erste Konzept beinhaltete Fumarsäuremonoethylester (198) als Ausgangsverbindung, dessen Überführung in die Verbindung 203 in geringen Ausbeuten von nur $31 \%$ gelang. Die Verwendung von Meerwein-Salz sollte nun ermöglichen, ein Lactimether-Intermediat 209 zu bilden, welches dann in einer [4+2]-Cycloaddition das Chinolin 210 liefern sollte. Dieser Versuch war jedoch nicht erfolgreich.

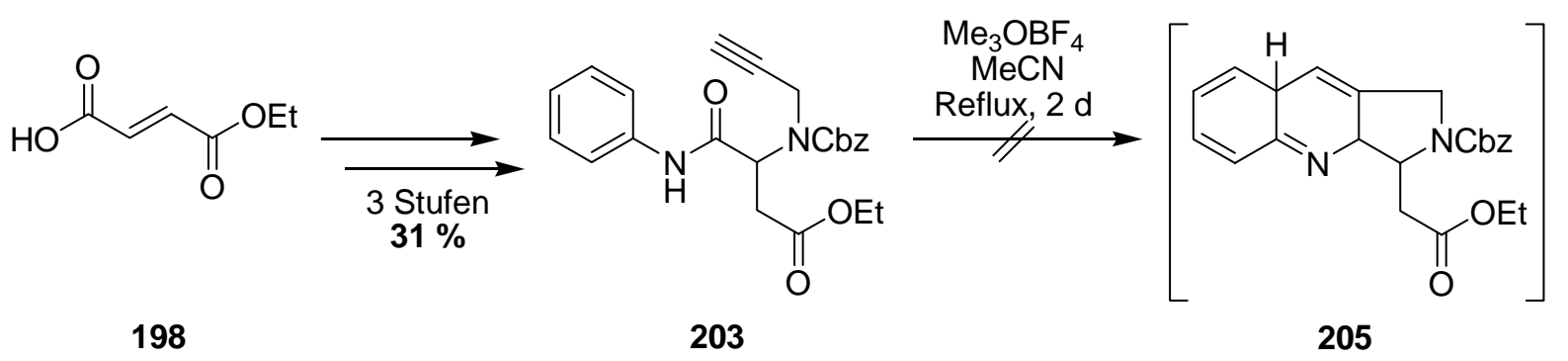

Abbildung 85: Versuche zur Synthese von 205.

In einer alternativen Synthesestrategie von 130 wurde die $\beta$-Aminosäure 207 in zwei Schritten in einer Ausbeute von 98 \% zum Cbz-geschützten Amin 2209 umgesetzt. Darauf aufbauend sollte in einer Michael-Additions-, Substitutions- und Decarboxylierungs-Sequenz Verbindung 212 dargestellt werden. Allerdings konnte diese Transformation trotz Variation der Reaktionsbedingungen nicht erfolgreich ausgeführt werden (Abbildung 86). 


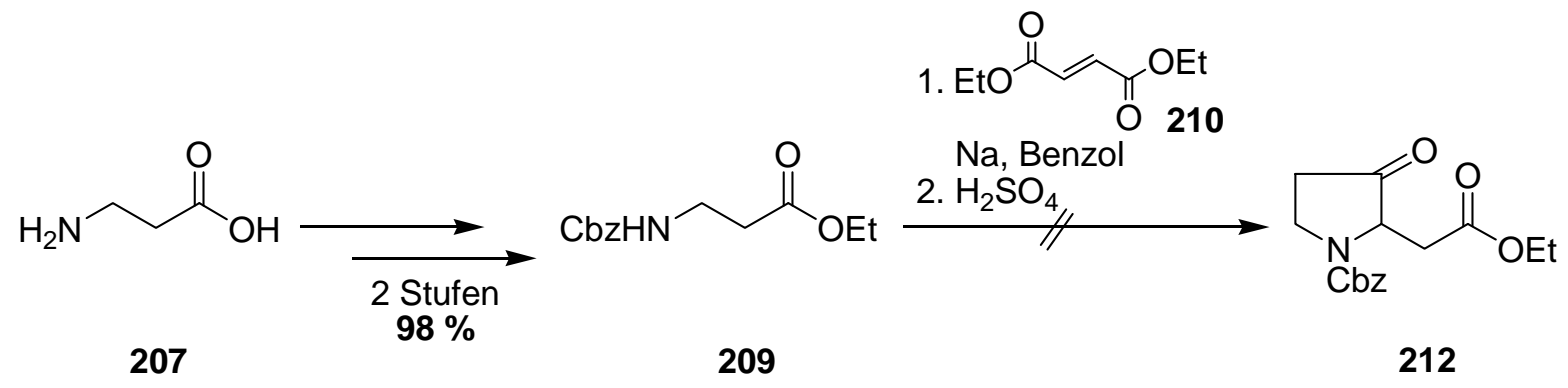

Abbildung 86: Versuche für die Synthese von 212.

Aufgrund der Schwierigkeiten, die bei der Synthese des Aldehyds 130 deutlich geworden waren, sollte ein Modellsystem aufgebaut werden. Dazu wurde das Isochinolin-Derivat 218 in einer 5-stufigen Synthese aus 213 in $28 \%$ Ausbeute hergestellt.<smiles>CCOC(=O)CC(=O)NCCc1ccccc1</smiles>

218

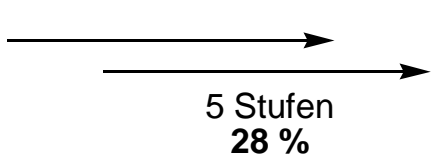

$28 \%$<smiles>[R16]CC1c2ccccc2CCN1C(=O)O</smiles>

Abbildung 87: Synthese des Modellsystems 218.

Die Durchführbarkeit der Domino-Knoevenagel-hetero-Diels-Alder-Reaktion für die Totalsynthese von Campthotecin (44) setzt die Darstellung des Silylenolethers 129 voraus. Dazu wurden gleich mehrere Ansätze gleichzeitig verfolgt. Ziel war es, die Alkohol-Funktion des 2-Hydroxybuttersäuremethylesters (219) mit TBSCl zu schützen, anschliessend ein Enolat zu erzeugen, um dieses im letzten Schritt zu Verbindung 129 silylieren. Eine Umwandlung von 219 und 223 in 129 gelang jedoch nicht. 


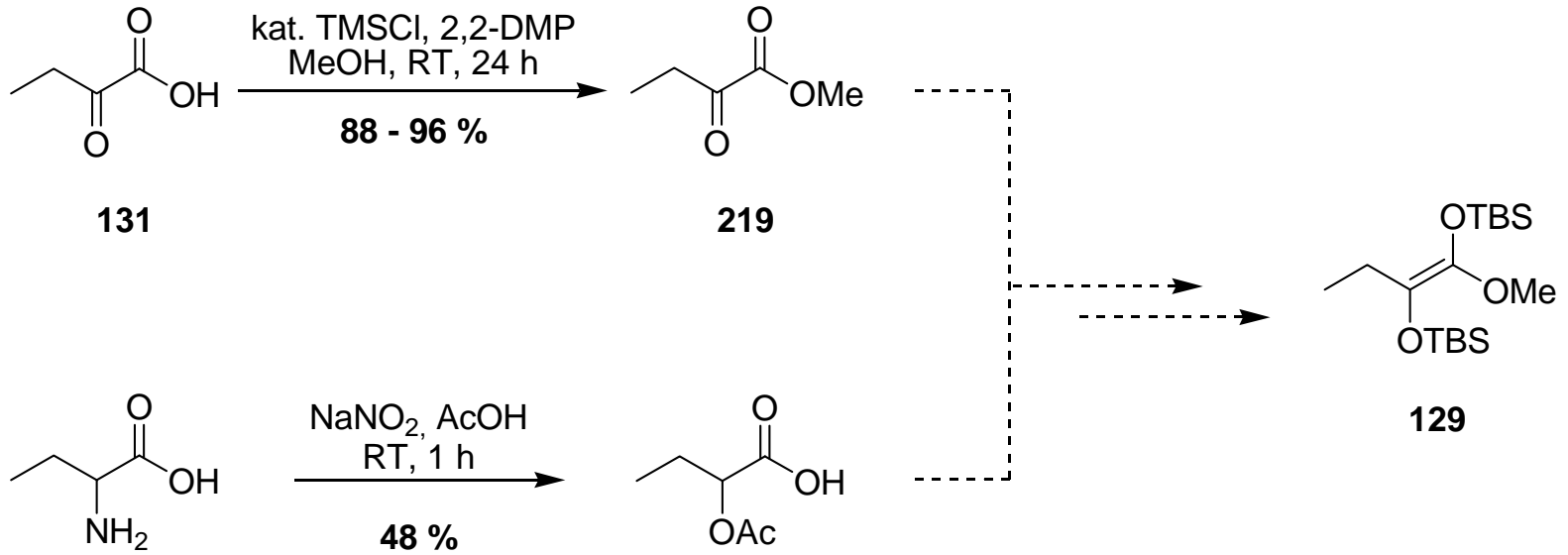

222

223

Abbildung 88: Versuche zur Synthese des Silylenolethers 129 über zwei Strategien. 


\section{EXPERIMENTELLER TEIL}

\section{Allgemeine Arbeitstechniken}

Die Umsetzungen wurden, soweit erforderlich, in mit Septumkappen bzw. Argon-TStück ausgestatteten ausgeheizten Glasapparaturen unter leichtem Argon-Überdruck durchgeführt. Die Lösungsmittel wurden entsprechend den üblichen Laboratoriumsmethoden getrocknet bzw. vorgetrocknet und destilliert. Kommerziell erhältliche Produkte wurden, sofern nicht anders angegeben, ohne weitere Reinigung eingesetzt. Das Entgasen von Lösungsmitteln geschah durch längeres Einleiten eines ArgonGasstroms und deren Entfernung im Vakuum mittels eines Rotationsverdampfers, dessen Wasserbadtemperatur dem jeweiligen Lösungsmittel sowie der Empfindlichkeit der Substanz angepasst war. Die Reaktionsführung bei tiefen Temperaturen erfolgte unter Verwendung üblicher Kühlmischungen (Trockeneis/Aceton; Natriumchlorid/Eis). Umsetzungen bei Temperaturen oberhalb des Lösungsmittel-Siedepunkts wurden in druckfesten Kolben mit Schraubverschluss durchgeführt.

\section{Verwendete Geräte:}

Schmelzpunkte: Die Bestimmung der Schmelzpunkte erfolgte mit dem Schmelzpunktbestimmungsgerät Modell FP 61 der Firma Mettler. Die angegebenen Werte sind nicht korrigiert.

Drehwerte: Drehwerte wurden in einem Polarimeter der Firma Perkin-Elmer, Modell 241 , gemessen.

Infrarotspektren: Die Aufnahme der IR-Spektren erfolgte mit dem Modell Vektor 22 der Firma Bruker. Nicht kristalline Substanzen wurden als Film zwischen NaCl- 
Platten, kristalline Proben als KBr-Presslinge gemessen. Zur Kalibrierung diente die Polystyrolbande bei $1601 \mathrm{~cm}^{-1}$.

UV/VIS-Spektren: UV-Spektren wurden mit dem Modell Lambda 2 der Firma Perkin-Elmer aufgenommen.

${ }^{1}$ H-NMR-Spektren: Die ${ }^{1}$ H-NMR-Spektren wurden mit den Modellen Mercury-200 (200 MHz), Mercury-300, Unity-300 (je $300 \mathrm{MHz)}$ und Inova-500 (500 MHz) sowie Unity Inova-600 (600 MHz) der Firma Varian aufgenommen. Die chemischen Verschiebungen sind in den Einheiten der $\delta$-Skala angegeben. Referenziert wurde bei nicht silylierten Verbindungen auf Tetramethylsilan (TMS, $\delta=0.00 \mathrm{ppm}$ ) als internen Standard oder auf das jeweilige Lösungsmittel. Zur Kennzeichnung der Signalmultiplizitäten wurden folgende Abkürzungen verwendet: $\mathrm{s}=$ Singulett, $\mathrm{s}_{\mathrm{b}}=$ breites Singulett, $\mathrm{d}=$ Dublett, $\mathrm{t}=$ Triplett, $\mathrm{q}=$ Quartett, quin $=$ Quintett, $\mathrm{dd}=$ Dublett vom Dublett, $\mathrm{dt}=$ Dublett vom Triplett, $\mathrm{m}=$ Multiplett, $\mathrm{m}_{\mathrm{c}}=$ zentriertes Multiplett. Phenylsubstituenten wurden mit der Abkürzung $\mathrm{Ph}$ und aromatische Systeme mit $\mathrm{Ar}$ gekennzeichnet. Signale, deren Zuordnung nicht eindeutig war, tragen den Index *. Im Fall diastereotoper Protonen wurde dem tieffeldverschobeneren Signal ein teifgestelltes A und dem hochfeldverschobenerem Signal ein tiefgstelltes B zugeordnet. Die Spektren wurden als solche erster Ordnung interpretiert. Die Kopplungskonstanten $J$ sind in Hertz (Hz) aufgeführt.

${ }^{13}$ C-NMR-Spektren: Die ${ }^{13}$ C-NMR-Spektren wurden mit den Modellen Mercury-200 (50.3 MHz), Mercury-300 und Unity-300 (je 75.5 MHz) sowie Unity Inova-600 $(150 \mathrm{MHz})$ der Firma Varian gemessen. Referenziert wurde bei nicht silylierten Verbindungen auf Tetramethylsilan (TMS, $\delta=0.00 \mathrm{ppm}$ ) als internen Standard oder auf das jeweilige Lösungsmittel. Die chemischen Verschiebungen sind den ${ }^{1} \mathrm{H}-$ breitbandentkoppelten Spektren entnommen, die Signalmultiplizitäten wurden in multiplett-selectio-Experimenten (APT-Plusfolge) bestimmt. Die Abkürzung Ph gilt 
Phenylsubstituenten und Ar wurde für Aromaten verwendet. Unsichere Zuordnungen sind durch den Index * ausgezeichnet.

Massenspektren: Zur Aufnahme der EI-Spektren diente ein doppelfokussierendes Sektorfeld-Massenspektrometer MAT 95 der Firma Finnigan. ESI-Spektren wurden mit einem Triple-Stage-Quadrupol-Instrument TSQ 7000 oder einem Ion-TrapMassenspektrometer LCQ der Firma Finnigan aufgenommen. Die angegebenen realtiven Peakintensitäten beziehen sich auf den Basispeak $(I=100 \%)$. Die Messung der ESI-HRMS-Spektren erfolgte an einem 7 Tesla-Fourier Transform Ion Cyclotron Resonance (FTICR)-Massenspektrometer APEX IV der Firma Bruker, das mit einer Apollo-Quelle dergleichen Firma und einer Spritzenpumpe 74900 Series der Firma Cole-Parmer ausgestattet ist. Der Fluss der Spritzpumpe betrug $2 \mu \mathrm{L} / \mathrm{min}$. Aufnahme und Auswertung der Spektren wurde mit den Programm XMASS vorgenommen.

Ultraschallbad: Für die Durchführung der Domino-Reaktion wurde das Modell Sonorex RK102 der Firma Bandelin verwendet.

Elementaranalyse: Die Elementaranalysen wurden im Mirkoanalytischen Labor des Institutes für Organische und Biomolekulare Chemie der Universität Göttingen durchgeführt.

\section{Chromatographische Methoden:}

Dünnschichtchromatographie (DC): Zur dünnschichtchromatographischen Kontrolle der Reaktionen kamen DC-Fertigfolien Alugram SIL G/UV 254 , Schichtdicke $0.25 \mathrm{~mm}$, der Firma Macherey-Nagel GmbH \& Co. KG zum Einsatz. Angegeben sind $\mathrm{R}_{\mathrm{f}}$-Werte (Laufhöhe relativ zur Laufmittelfront). Neben der UV-Detektion diente Vanillin-Schwefelsäure-Lösung (900 mL MeOH, $100 \mathrm{~mL}$ Eisessig, $30 \mathrm{~mL}$ konz. $\mathrm{H}_{2} \mathrm{SO}_{4}$ und $5 \mathrm{~g}$ Vanillin) als Anfärbereagenz. Die verwendeten Eluentien wurden wie 
folgt abgekürzt: PE (Pentan), E (Ether), $\mathrm{CH}_{2} \mathrm{Cl}_{2}$ (Dichlormethan), EE (Essigsäureethylester) und $\mathrm{MeOH}$ (Methanol).

Säulenfiltration und Säulenchromatographie (SC): Für die säulenchromatographische Trennung unter erhöhtem Druck (Flashchromatographie bei Drücken von 120-150 kPa) wurde Kieselgel der Firma Merck (Korngröße 0.040-0.063 mm) verwendet.

Hochdruckflüssigkeitschromatographie (HPLC): Die analytischen Trennungen erfolgten mit einer HPLC-Anlage der Firma Kontron mit Lösungsmittelpumpen des Typs 422, Programer, Mischkammer M800 und Steuerung. Das Computerprogramm und die Software zur Auswertung der Daten stammten ebenfalls von der Firma Kontron (Kroma System). Des Weiteren waren ein automatischer Probenwechsler (Autosampler 360) und ein UV-Detektor (Diode Array Detector 440) angeschlossen. Zur Trennung kam eine Fertigsäule Chiracel OD $(250 \times 4.6 \mathrm{~mm})$ (Säule A) der Firma Baker zum Einsatz. Die Lösungsmittelgemische wurden von der verwendeten Anlage auf der Hochdruckseite erzeugt. 


\section{Allgemeine Arbeitsvorschriften (AAV)}

\section{Silylierung von Alkoholen (AAV 1)}

$\mathrm{Zu}$ einer Lösung des Alkohols (1.0 Äq.), Imidazol (1.2 Äq.) und einer katalytischen Menge DMAP in DMF (2.8 mL pro mmol Alkohol) tropft man Silylchlorid (1.2 Äq.). Der Ansatz wird bei Raumtemperatur gerührt und der Fortgang der Reaktion mittels DC verfolgt. Nach vollständigem Umsatz (18 h) versetzt man den Reaktionsansatz mit gesättigter $\mathrm{NaHCO}_{3}$-Lösung $\left(7.0 \mathrm{~mL}\right.$ pro mmol) und $\mathrm{H}_{2} \mathrm{O}(7.0 \mathrm{~mL}$ pro mmol) und extrahiert die wässrige Phase mit $\mathrm{Et}_{2} \mathrm{O}(4 \times 7.0 \mathrm{~mL}$ pro $\mathrm{mmol})$. Die vereinigten organischen Phasen werden über $\mathrm{Na}_{2} \mathrm{SO}_{4}$ getrocknet und das Lösungsmittel im Vakuum entfernt. Das Rohprodukt wird durch Säulenchromatographie gereinigt.

\section{DIBAH-Reduktion von Estern zu Aldehyden (AAV 2)}

Essigsäureester (1.0 Äq.) wird in wasserfreiem $\mathrm{CH}_{2} \mathrm{Cl}_{2}(10 \mathrm{~mL}$ pro mmol) gelöst und bei $-78{ }^{\circ} \mathrm{C}$ eine auf $-78{ }^{\circ} \mathrm{C}$ vorgekühlte DIBAH-Lösung (1.1-1.6 Äq., $1 \mathrm{M}$ in Hexan) über eine Transferkanüle zugetropft. Der Ansatz wird bei $-78^{\circ} \mathrm{C}$ gerührt und der Fortgang der Reaktion mittels DC verfolgt. Nach vollständigem Umsatz (2 h) gibt man bei $-78{ }^{\circ} \mathrm{C}$ eine Mischung aus $\mathrm{MeOH}$ und $2 \mathrm{~N} \mathrm{HCl}(9: 1,3.0 \mathrm{~mL}$ pro mmol Ester) vorsichtig zur Reaktionsmischung, lässt zügig mittels Wasserbad auf Raumtemperatur erwärmen und nimmt in gesättigter $\mathrm{NH}_{4} \mathrm{Cl}$-Lösung (10 mL pro mmol Ester) auf. Man extrahiert zweimal mit dem gleichen Volumen $\mathrm{CH}_{2} \mathrm{Cl}_{2}$, wäscht die vereinigten organischen Phasen mit gesättigter $\mathrm{NaHCO}_{3}$-Lösung, trocknet über $\mathrm{Na}_{2} \mathrm{SO}_{4}$ und entfernt das Lösungsmittel im Vakuum. Das Rohprodukt wird durch Säulenchromatographie gereinigt.

\section{LiAlH $_{4}$-Reduktion von Estern zu Alkoholen (AAV 3)}

$\mathrm{Zu}$ einer Suspension von $\mathrm{LiAlH}_{4}$ (3.0 Äq.) in THF (4.5 mL pro mmol) tropft man bei $0{ }^{\circ} \mathrm{C}$ eine Lösung des Essigsäureesters (1.0 Äq.) in THF (2.5 mL pro mmol) dazu. Der Ansatz wird bei Raumtemperatur gerührt und der Fortgang der Reaktion mittels DC verfolgt. Nach vollständigem Umsatz (0.5-3 h) wird der Reaktionsansatz mit $\mathrm{MgSO}_{4}$ $(0.25 \mathrm{mg})$ und $\mathrm{H}_{2} \mathrm{O}(156 \mu \mathrm{L})$ bei $0{ }^{\circ} \mathrm{C}$ versetzt und so lange gerührt, bis sich ein feiner 
weißer Niederschlag bildet. Dieser wird abfiltriert und je zweimal mit dem gleichen Volumen $\mathrm{Et}_{2} \mathrm{O}$ und $\mathrm{EE}$ gewaschen. Die vereinigten organischen Phasen werden über $\mathrm{Na}_{2} \mathrm{SO}_{4}$ getrocknet und das Lösungsmittel im Vakuum entfernt. Das Rohprodukt wird durch Säulenchromatographie gereinigt.

\section{$\mathrm{KMnO}_{4}$-Oxidatin von Aminen zu Iminen (AAV 4)}

$\mathrm{Zu}$ einer Lösung des Amins (1.0 Äq.) in THF bzw. $\mathrm{CH}_{3} \mathrm{CN} / \mathrm{MeOH}$ (2:1) $(15 \mathrm{~mL}$ pro mmol) gibt man bei $0{ }^{\circ} \mathrm{C}$ pulverisiertes $\mathrm{KMnO}_{4}$ (2.1-6.3 Äq.) in kleinen Portionen dazu. Der Ansatz wird weiter bei $0{ }^{\circ} \mathrm{C}$ gerührt und der Fortgang der Reaktion mittels DC verfolgt. Nach vollständigem Umsatz (1-2 h) wird der gebildete Braunstein abgenutscht und das Lösungsmittel im Vakuum entfernt. Das Rohprodukt wird direkt weiter umgesetzt.

\section{Domino-Knoevenagel-hetero-Diels-Alder-Reaktion (AAV 5)}

Aldehyd (1.0 Äq.), Meldrum-Säure (1.2 Äq.), Enolether (3.0 Äq.) und wenige Kristalle Ethylendiammoniumdiacetat (EDDA) werden in einem Rundhochdruckkolben in Benzol (1.0 mL pro mmol Aldehyd) gelöst. Der Ansatz wird unter Argonatmosphäre dicht verschlossen und die rote Lösung bei $60{ }^{\circ} \mathrm{C}$ gerührt. Der Fortgang der Reaktion wird mittels DC verfolgt. Nach vollständigem Umsatz (8-20 h) wird in jedem Fall eine rötlich-klare Reaktionslösung erhalten, die entweder durch Säulenchromatographie an desaktiviertem Kieselgel gereinigt oder direkt weiter umgesetzt wird.

\section{Hydrogenolyse von Lactonacetalen (AAV 6)}

Lactonacetal (1.0 Äq.) wird in $\mathrm{MeOH}(20 \mathrm{~mL}$ pro mmol) gelöst und unter Rühren festes trockenes $\mathrm{K}_{2} \mathrm{CO}_{3}$ hinzu gegeben. Der Ansatz wird 30-50 min bei Raumtemperatur gerührt und die Reaktionslösung anschließend mittels einer Spritze $\mathrm{zu}$ einer zuvor mit $\mathrm{H}_{2}$ gesättigten Suspension von Palladium auf Kohle $(10 \% \mathrm{Pd}$; 50 Gewicht- $\%$ des Katalysators bezogen auf Lactonacetal) in $\mathrm{MeOH}(10 \mathrm{~mL}$ pro $\mathrm{mmol}$ Lactonacetal) gegeben. Der Ansatz wird bei Raumtemperatur gerührt und der Fortgang der Reaktion mittels DC verfolgt. Nach vollständigem Umsatz (8-20 h) wird der Katalysator abfiltriert und mit $\mathrm{MeOH}(3 \times 20 \mathrm{~mL}$ pro mmol) ausgewaschen. Nach 
Entfernen des Lösungsmittels im Vakuum wird das Rohprodukt durch Säulenchromatographie gereinigt.

\section{Katalytische asymmetrische Transferhydrierung nach Noyori (AAV 7) Synthese des Katalysators 159}

In einem geschlossenen Kolben wird eine Lösung von Dichlor-( $p$-cymol)ruthenium(II) Dimer (1.0 Äq.), 1,2-(R,R)-N-Tosyl-1,2-diphenyldiamin (2.2 Äq.) und $\mathrm{NEt}_{3}$ (4.0 Äq.) in DMF bzw. $\mathrm{CH}_{3} \mathrm{CN}(0.01 \mathrm{~mL}$ pro $\mu \mathrm{mol}$ Ru-Dimer) für $60 \mathrm{~min}$ bei einer Temperatur von $80^{\circ} \mathrm{C}$ gerührt. Die warme Lösung wird direkt in der TransferHydrierung eingesetzt.

\section{Synthese des Katalysators 125}

In einem geschlossenen Kolben wird eine Lösung von Dichlor- $\left(\eta^{6}\right.$-benzol)ruthenium(II) Dimer (1.0 Äq.) und 1,2-(R,R)-N-Tosyl-1,2-diphenyldiamin (2.2 Äq.) in $\mathrm{CH}_{3} \mathrm{CN}(0.01-0.5 \mathrm{~mL}$ pro $\mu \mathrm{mol} \mathrm{Ru}-\mathrm{Dimer})$ für $5 \mathrm{~min}$ bei Raumtemperatur gerührt. Die Lösung wird direkt in der Transfer-Hydrierung eingesetzt.

\section{Transfer-Hydrierung}

$\mathrm{Zu}$ einer Lösung des Imins (1.0 Äq.) in DMF bzw. $\mathrm{CH}_{3} \mathrm{CN}(2.0-6.0 \mathrm{~mL}$ pro mmol Imin) wird Katalysator $159 \mathrm{bzw}$. Katalysator 125 gegeben, die Lösung auf $0{ }^{\circ} \mathrm{C}$ gekühlt. Und eine Mischung von Ameisensäure/ $\operatorname{Net}_{3}$ (5:2, $0.5 \mathrm{~mL}$ pro mmol Imin) tropfenweise hinzu gegeben. Der Ansatz wird bei Raumtemperatur gerührt und der Fortgang der Reaktion mittels DC verfolgt. Nach vollständigem Umsatz (8-48 h) wird gesättigte $\mathrm{Na}_{2} \mathrm{CO}_{3}$-Lösung zugegeben und gerührt, bis sich pH-Wert von 10-12 eingestellt hat. Man extrahiert dreimal mit dem doppelten Volumen Essigester, wäscht die vereinigten organischen Phasen mit gesättigter $\mathrm{NaCl}$-Lösung, trocknet über $\mathrm{Na}_{2} \mathrm{SO}_{4}$ und entfernt das Lösungsmittel im Vakuum. Das Rohprodukt wird durch Säulenchromatographie gereinigt. 
A) Versuche zur Synthese von Yohimbin (9)

\section{Synthese des chiralen Hydrierungskatalysatorliganden}

\section{1. (1S,2S)- $N$-Tosyl-1,2-diphenylethylendiamin (224)}<smiles>N[C@@H](c1ccccc1)C([PbH])N[TeH3]</smiles>

$\mathrm{Zu}$ einer Lösung von (1S,2S)-1,2-Diphenylethylendiamin (258 mg, $1.22 \mathrm{mmol}$, 1.0 Äq.) und $\mathrm{NEt}_{3}(0.52 \mathrm{~mL}, 1.0$ Äq.) in THF (10.0 mL, $8.50 \mathrm{~mL}$ pro mmol) gab man bei $0{ }^{\circ} \mathrm{C}$ tropfenweise eine Lösung von TosCl (233 mg, $1.22 \mathrm{mmol}, 1.0$ Äq.) in THF (2.70 mL, $2.21 \mathrm{~mL}$ pro mmol Chlorid) und ließ $16 \mathrm{~h}$ bei Raumtemperatur rühren. Die Reaktion wurde mit gesättigter $\mathrm{NaCl}$-Lösung gewaschen und über $\mathrm{Na}_{2} \mathrm{SO}_{4}$ getrocknet. Das Lösungsmittel wurde im Vakuum entfernt und das Rohprodukt durch Säulenchromatographie (EE) gereinigt. Man erhielt die Titelverbindung (256 mg, $0.70 \mathrm{mmol}, 58 \%$ ) als weißen Feststoff.

$\mathbf{R}_{\mathbf{f}}=0.65(\mathrm{EE})$.

$[\alpha]_{\mathbf{D}}^{20}=33.0^{\circ}\left(c=0.2, \mathrm{CHCl}_{3}\right)$.

$\mathbf{U V}\left(\mathrm{CH}_{3} \mathrm{CN}\right): \lambda_{\max }(\lg \varepsilon): 192.5 \mathrm{~nm}(2.442)$.

IR $(\mathrm{KBr}): v=3344 \mathrm{~cm}^{-1}(\mathrm{NH}), 1329\left(\mathrm{CH}_{3}\right), 1155,698,670,571,543$.

${ }^{1} \mathrm{H}-\mathrm{NMR}\left(300 \mathrm{MHz}, \mathrm{CDCl}_{3}\right): \delta=2.35\left(\mathrm{~s}, 3 \mathrm{H}, \mathrm{Ar}-\mathrm{CH}_{3}\right), 4.26\left(\mathrm{~d}, 1 \mathrm{H},-\mathrm{CHPh}-\mathrm{NH}_{2}, J=\right.$ $6.0 \mathrm{~Hz}), 4.48$ (d, $1 \mathrm{H},-\mathrm{CHPh}-\mathrm{NHAr}, J=6.0 \mathrm{~Hz}), 6.94(\mathrm{~d}, 2 \mathrm{H}, m \mathrm{marH}, J=8.0 \mathrm{~Hz}$ ), 7.09 (s, 5 H, Ph-H), 7.14 (s, 5 H, Ph-H), 7.32 (d, 2 H, oArH, $J=8.5$ Hz). 


\section{2. $\quad(1 R, 2 R)-N$-Tosyl-1,2-diphenylethylendiamin (224)}<smiles>CN[C@@H](c1ccccc1)C(N)c1ccccc1</smiles>

(1R,2R)-N-Tosyl-1,2-diphenylethyldiamin (450 mg, $2.12 \mathrm{mmol}, 1.0$ Äq.) wurde analog zu (1S,2S)-1,2-Diphenylethylendiamin synthetisiert. Man erhielt die Titelverbindung (694 mg, $1.90 \mathrm{mmol}, 90 \%$ ) als weißen Feststoff. Die spektroskopischen Daten stimmen mit denen von Verbindung (1S,2S)-224 überein.

$[\alpha]_{\mathbf{D}}^{20}=-41.8^{\circ}\left(c=0.11, \mathrm{CHCl}_{3}\right)$

\subsection{Ru-Dimer für die Synthese von Katalysator 125}<smiles>Cl[Te](Cl)(Cl)(Cl)(Cl)c1ccccc1</smiles>

Eine Mischung aus $\mathrm{RuCl}_{3} \cdot \mathrm{H}_{2} \mathrm{O}(600 \mathrm{mg}, 2.9 \mathrm{mmol}, 1.0$ Äq. $41 \% \mathrm{Ru})$, Ethanol $(8.0 \mathrm{~mL})$ und 1,4-Cycloheandien $(6.0 \mathrm{~mL})$ wurde für $6 \mathrm{~h}$ refluxiert. Der entstandene Niederschlag wurde abgenutscht und unter vermindertem Druck getrocknet. Man erhielt den Ruthenium-Dimer (527 mg, 1.05 mmol, 87 \%) als orange-rotes Pulver. 


\section{Synthese des Aldehyds 121}

\subsection{Carbethoxysäure (126)}<smiles>CCOC(=O)CC(=O)C(=O)O</smiles>

$\mathrm{Zu}$ einer Lösung von Diethylnatriumoxalacetat (42.0 g, $200 \mathrm{mmol}, 1.0$ Äq.) in Wasser (400 mL) wurde tropfenweise $6 \mathrm{~N}$ Natriumhydroxid $(33.4 \mathrm{~mL}, 1.0$ Äq.) zugegeben und die Reaktionsmischung $3 \mathrm{~h}$ bei Raumtemperatur gerührt. Man gab $6 \mathrm{~N}$ Salzsäure (70.0 mL, 2.1 Äq.) bei $0^{\circ} \mathrm{C}$ hinzu und extrahierte die wässrige Phase mit Diethylether $(8 \times 140 \mathrm{~mL})$ und Ethylacetat $(3 \times 140 \mathrm{~mL})$. Die vereinigten organischen Phasen wurden über $\mathrm{MgSO}_{4}$ getrocknet und das Lösungsmittel im Vakuum entfernt. Man erhielt die Titelverbindung $(28.0 \mathrm{~g}, 175 \mathrm{mmol}, 88 \%)$ als orangene Suspension, bestehend aus der kristallinen Enolform und der öligen Ketoform.

UV $\left(\mathrm{CH}_{3} \mathrm{CN}\right): \lambda_{\max }(\lg \varepsilon)=194.0 \mathrm{~nm}(0.196), 211.5$ (0.126), 257.0 (0.353).

IR $(\mathrm{KBr}): v=2986 \mathrm{~cm}^{-1}(\mathrm{CH}), 1729(\mathrm{C}=\mathrm{O}), 1415\left(\mathrm{CH}_{2}\right), 1217,1026\left(\mathrm{OCH}_{2}\right), 853$, 787.

${ }^{1} \mathbf{H}-\mathbf{N M R}\left(300 \mathrm{MHz}, \mathrm{CDCl}_{3}\right): \delta=1.22$ (t (Enolform), $\left.2.4 \mathrm{H}, \mathrm{CH}_{3}, J=7.0 \mathrm{~Hz}\right), 1.29$ (t (Ketoform), 3.0 H, $\mathrm{CH}_{3}, J=7.0 \mathrm{~Hz}$ ), 4.09 (q (Enolform), $1.5 \mathrm{H}, \mathrm{CH}_{2}, J=7.0 \mathrm{~Hz}$ ), 4.25 (q (Ketoform), $2 \mathrm{H}, \mathrm{CH}_{2}, J=7.0 \mathrm{~Hz}$ ), 5.93 (s (Enolform), $0.6 \mathrm{H}, \mathrm{CH}$ ).

${ }^{13}$ C-NMR (50.3 MHz, $\left.\mathrm{CDCl}_{3}\right): \delta=14.30,14.36\left(\mathrm{OCH}_{2} \mathrm{CH}_{3}\right), 47.72\left(\mathrm{OCH}_{2} \mathrm{CH}_{3}\right)$, 61.74, $61.90\left(\mathrm{CH}_{2}\right), 96.89(\mathrm{CH}), 169.98(\mathrm{COOH}), 170.67\left(\mathrm{C}=\mathrm{O}-\mathrm{OCH}_{2} \mathrm{CH}_{3}\right), 172.36$ $(\mathrm{C}=\mathrm{O}-\mathrm{COOH})$.

MS $\left(\mathrm{DCI}-\mathrm{NH}_{3}\right): m / z(\%)=178(29)[\mathrm{M}+18]^{+}, 195(11)[\mathrm{M}+17+18]^{+}$.

$\mathrm{C}_{6} \mathbf{H}_{8} \mathbf{O}_{5}(160.04)$ 


\subsection{1-Carbethoxymethyl-1,2,3,4-tetrahydro- $\beta$-carbolin (123)}<smiles>CCOC(=O)CC1NCCc2c1[nH]c1ccccc21</smiles>

$\mathrm{Zu}$ einer refluxierenden Lösung von Tryptamin Hydrochlorid $(25.0 \mathrm{~g}, 127 \mathrm{mmol}$, 1.0 Äq.) in Ethanol $(400 \mathrm{~mL})$ wurde tropfenweise eine Lösung von Carbethoxypyruvinsäure (28.4 g, $178 \mathrm{mmol}, 1.4$ Äq) in Ethanol $(85 \mathrm{~mL})$ zugegeben und die Reaktionsmischung $40 \mathrm{~h}$ unter Rückfluss erhitzt. Die einsetzende Kristallisation wurde durch Übernachtstellen in den Kühlschrank vervollständigt. Der Niederschlag wurde abfiltriert, mit kaltem Diethylether gewaschen, in ges. $\mathrm{NaHCO}_{3}$ Lsg. (400 mL) aufgenommen und die Suspension so lange kräftig gerührt, bis sich der Feststoff vollständig aufgelöst hatte. Die wässrige Phase wurde mit Ethylacetat $(2 \times$ $400 \mathrm{~mL}$ ) extrahiert und die vereinigten organischen Phasen über $\mathrm{Na}_{2} \mathrm{SO}_{4}$ getrocknet. Das Lösungsmittel wurde im Vakuum entfernt und man erhielt die Titelverbindung (36.7 g, $142 \mathrm{mmol}, 80 \%$ ) als ein hellbraunes, klebriges Öl.

$\mathbf{R}_{\mathbf{f}}=0.55\left(\mathrm{MeOH} / \mathrm{EE} \mathrm{NEt}_{3}=1: 3: 1 \%\right)$.

UV $\left(\mathrm{CH}_{3} \mathrm{CN}\right): \lambda_{\max }(\lg \varepsilon)=195.0 \mathrm{~nm}$ (1.183), 226.5 (1.659), 280.5 (0.390), 289.0 $(0.314)$.

IR (KBr): $v=3400 \mathrm{~cm}^{-1}\left(\mathrm{~N}-\mathrm{H}\right.$ Indole), $2930(\mathrm{C}-\mathrm{H}), 1722(\mathrm{C}=\mathrm{O}), 1451\left(\mathrm{CH}_{2}\right), 1373$ $\left(\mathrm{CH}_{3}\right), 1157$ (C-O-Et), 1112, $1023\left(\mathrm{OCH}_{2}\right), 742(\mathrm{Ph}-\mathrm{H})$.

${ }^{1} \mathrm{H}-\mathrm{NMR}\left(300 \mathrm{MHz}, \mathrm{CDCl}_{3}\right): \delta=1.28\left(\mathrm{t}, 3 \mathrm{H}, \mathrm{CH}_{3}, J=7.0 \mathrm{~Hz}\right), 1.90\left(\mathrm{~s}_{\mathrm{b}}, 1 \mathrm{H}, \mathrm{NH}\right)$, $2.72\left(\mathrm{~m}, 2 \mathrm{H}, 4-\mathrm{H}_{2}\right), 2.79$ (d, $2 \mathrm{H}, 1^{\prime}-\mathrm{H}_{2}, J=7.0 \mathrm{~Hz}$ ), 3.07 (dt, $1 \mathrm{H}, 3-\mathrm{H}_{\mathrm{a}}, J=5.5,13.0$ $\mathrm{Hz}), 3.21\left(\mathrm{dt}, 1 \mathrm{H}, 3-\mathrm{H}_{\mathrm{b}}, J=5.5,13.0 \mathrm{~Hz}\right), 4.20\left(\mathrm{q}, 2 \mathrm{H}, 3\right.$ '- $\left.\mathrm{H}_{2}, J=7.0 \mathrm{~Hz}\right), 4.44$ (t, 1 H, 1-H, $J=7.0 \mathrm{~Hz}$ ), 7.10 (dtd, 2 H, H-6, H-7, $J=1.0,7.0,7.0$ Hz), 7.28 (d, 1 H, H-8, $J$ $=10 \mathrm{~Hz}), 7.48(\mathrm{~d}, 1 \mathrm{H}, \mathrm{H}-5, J=10 \mathrm{~Hz}), 8.64\left(\mathrm{~s}_{\mathrm{b}}, 1 \mathrm{H}, \mathrm{N}-\mathrm{H}\right.$ Indole $)$.

${ }^{13}$ C-NMR (50.3 MHz, $\mathrm{CDCl}_{3}$ ): $\delta=14.07$ (C-4'), 22.45 (C-4), 40.64 (C-1'), 41.79 (C3), 48.72 (C-1), 60.95 (C-3'), 108.87 (C-4a), 110.84 (C-8), 117.99 (C-5), 119.13 (C-7), 121.56 (C-6), 127.04 (C-4b), 134.86 (C-9a), 135.43 (C-8a), 173.03 (C=O). 
MS $\left(\mathrm{DCI}-\mathrm{NH}_{3}\right): m / \mathrm{z}(\%)=259(100)[\mathrm{M}+1]^{+}, 517(20)[2 \mathrm{M}+1]^{+}$.

$\mathbf{C}_{15} \mathbf{H}_{18} \mathbf{N}_{2} \mathbf{O}_{2}(258.14)$

\subsection{1-Carbethoxymethyl-2-benzyloxycarbonyl-1,2,3,4-tetrahydro- $\beta$-carbolin} (173)

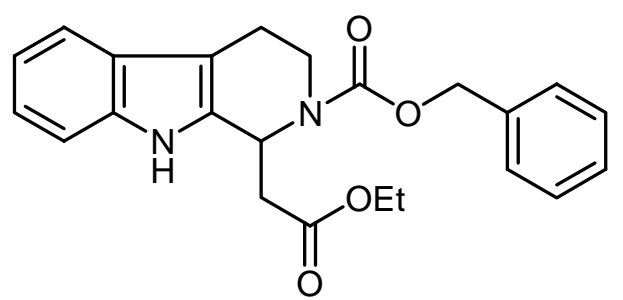

$\mathrm{Zu}$ einer Lösung von dem Ester $123(7.28 \mathrm{~g}, 28.0 \mathrm{mmol}, 1.0$ Äq.), Triethylamin (11.64 mL, $84 \mathrm{mmol}, 3.0$ Äq.) und katalytischen Mengen von DMAP in $\mathrm{CH}_{2} \mathrm{Cl}_{2}$ $(28 \mathrm{~mL})$ wurde bei $0{ }^{\circ} \mathrm{C}$ eine Lösung von Chlorameisensäurebenzylester $(4.78 \mathrm{~mL}$, $33.6 \mathrm{mmol}, 1.2$ Äq.) in $\mathrm{CH}_{2} \mathrm{Cl}_{2}(17 \mathrm{~mL})$ zugegeben und die Reaktionsmischung für $12 \mathrm{~h}$ bei Raumtemperatur gerührt. Man wusch mit Wasser $(45 \mathrm{~mL}), 2 \mathrm{~N}$ Salzsäure (45 mL), Wasser $(45 \mathrm{~mL})$, ges. $\mathrm{Na}_{2} \mathrm{CO}_{3}$-Lsg. (45 mL) und ges. NaCl-Lsg. (45 mL), trocknete die organische Phase über $\mathrm{Na}_{2} \mathrm{SO}_{4}$ und entfernte das Lösungsmittel im Vakuum. Man erhielt die Titelverbindung $9.8 \mathrm{~g}, 25.0 \mathrm{mmol}, 89 \%$ ) als ein hellbraunes, klebriges Öl.

$\mathbf{R}_{\mathbf{f}}=0.69(\mathrm{EE})$.

UV $\left(\mathrm{CH}_{3} \mathrm{CN}\right): \lambda_{\max }(\lg \varepsilon)=224.0$ (1.107), $273.0(0.237), 278.5$ (0.235), $289.0(0.176)$

IR (KBr): $v=3395 \mathrm{~cm}^{-1}$ (N-H Indol), $2981(\mathrm{CH}), 1699(\mathrm{C}=\mathrm{O}), 1424,1361\left(\mathrm{CH}_{3}\right)$, 1266, 1218 (O-Ar), 1100, $1020\left(\mathrm{OCH}_{2}\right), 742$ (1,2-disubst. Benzol), 699 (monosubst. Benzol).

${ }^{1}$ H-NMR (300 MHz, $\left.\mathrm{CDCl}_{3}\right): \delta=1.26\left(\mathrm{td}, 3 \mathrm{H}, \mathrm{CH}_{3}, J=3.5,7.0 \mathrm{~Hz}\right), 2.67-2.99(\mathrm{~m}, 4$ H, 4- $\mathrm{H}_{2}, 1$ ' $\left.-\mathrm{H}_{2}\right), 3.14\left(\mathrm{~m}_{\mathrm{c}}, 1 \mathrm{H}, 3-\mathrm{H}_{\mathrm{a}}\right), 4.20\left(\mathrm{~m}, 2 \mathrm{H}, \mathrm{CH}_{2}\right), 4.51$ (ddd, $1 \mathrm{H}, 1-\mathrm{H}, J=5.0$, 13.0, 39.0 Hz), 5.19 (s, $2 \mathrm{H}, \mathrm{Ph}-\mathrm{CH}_{2}$ ), 5.67 (ddd, $1 \mathrm{H}, 3-\mathrm{H}_{\mathrm{b}}, J=4.0,8.0,25.0 \mathrm{~Hz}$ ), 7.08 (dd, $1 \mathrm{H}, 8-\mathrm{H}, J=7.1,7.1 \mathrm{~Hz}), 7.16(\mathrm{ddd}, 1 \mathrm{H}, 9-\mathrm{H}, J=1.0,7.1,7.1 \mathrm{~Hz}), 7.32-7.42$ 
(m, 6 H, 7-H, Ph-H), 7.47 (dd, $1 \mathrm{H}, 10-\mathrm{H}, J=7.1,7.1 \mathrm{~Hz}), 8.75,8.91$ (2 s $\mathrm{br}, 1 \mathrm{H}$, Indol$\mathrm{NH})$.

${ }^{13}$ C-NMR (50.3 MHz, $\left.\mathrm{CDCl}_{3}\right): \delta=14.05\left(\mathrm{CH}_{3}\right), 21.05(\mathrm{C}-4), 39.00$ (C-1'), $39.06(\mathrm{C}-$ 2'’), 39.29 (C-3), 47.52 (C-1), $61.16\left(\mathrm{CH}_{2}\right), 108.12$ (C-5), 111.06 (C-10), 118.03 (C7), 119.29 (C-9), 121.92 (C-8), 126.25 (C-6), 127.82, 128.03, 128.48, 132.95 (Ph-C), 135.59 (C-11), 136.49 (C-13), 155.01 (C-1'’), 172.90 (C-2').

MS $\left(\mathrm{DCI}-\mathrm{NH}_{3}\right): m / z(\%)=393(70)[\mathrm{M}+1]^{+}, 410(62)[\mathrm{M}+18]^{+}$.

$\mathbf{C}_{23} \mathbf{H}_{24} \mathbf{N}_{2} \mathbf{O}_{4}(392.17)$

\subsection{Benzyl-1-(2-oxoethyl)-3,4-dihydro-1H-pyrido[3,4-b]indol-2(9H)-carboxylat} (121)<smiles>O=CCC1c2[nH]c3ccccc3c2CCN1C(=O)OCc1ccccc1</smiles>

Analog AAV 2 setzte man den Ester 173 (8.6 g, 21.9 mmol, 1.0 Äq.) mit DIBAH (33.0 mL, $32.9 \mathrm{mmol}, 1.5 \mathrm{Äq} ., 1 \mathrm{M}$ in Hexan) bei $-78{ }^{\circ} \mathrm{C}$ in $\mathrm{CH}_{2} \mathrm{Cl}_{2}(219 \mathrm{~mL}$,) innerhalb von 2 h um. Die Reinigung erfolgte durch Säulenchromatographie (EE/P). Man erhielt die Titelverbindung (4.92 g, $14.1 \mathrm{mmol}, 65 \%$ ) als einen farblosen Schaum.

$\mathbf{R}_{\mathbf{f}}=0.61(\mathrm{EE} / \mathrm{P}=1: 1)$.

Smp. $=65^{\circ} \mathrm{C}$

UV $\left(\mathrm{CH}_{3} \mathrm{CN}\right): \lambda_{\max }(\lg \varepsilon)=223$ (4.534), 273 (3.862), 279 (3.859), 289 (3.888).

IR (KBr): $v=3395 \mathrm{~cm}^{-1}(\mathrm{NH}), 1692(\mathrm{C}=\mathrm{O}), 698$ (Phenyl-H).

${ }^{1} \mathbf{H}-\mathbf{N M R}\left(500 \mathrm{MHz}, \mathrm{CDCl}_{3}\right): \delta=2.66-2.92\left(\mathrm{~m}, 2 \mathrm{H}, 4-\mathrm{H}_{2}\right), 3.02-3-21$ (m, $3 \mathrm{H}, 1$ '$\mathrm{H}_{2}, 3-\mathrm{H}_{\mathrm{a}}$ ), 4.46, 4.77 (2 dd (Rotamere), $\left.1 \mathrm{H}, 4-\mathrm{H}_{\mathrm{b}}, J=5.0,12.0 \mathrm{~Hz}\right), 5.16\left(\mathrm{~m}_{\mathrm{c}}, 2 \mathrm{H}, \mathrm{Ph}-\right.$ $\mathrm{CH}_{2}$ ), 5.68, 5.75 (2 d (Rotamere), $1 \mathrm{H}, 1-\mathrm{H}, J=8.0 \mathrm{~Hz}$ ), 7.08 (ddd, $1 \mathrm{H}, 6-\mathrm{H}, J=1.0$, 7.0, 7.0 Hz), 7.15 (ddd, $1 \mathrm{H}, 7-\mathrm{H}, J=1.0,7.0,7.0 \mathrm{~Hz}$ ), $7.27-7.40$ (m, $6 \mathrm{H}, 8-\mathrm{H}$, Ar- 
H), $7.44(1 \mathrm{H}, 5-\mathrm{H}), 8.43,8.57$ (2 $\mathrm{s}_{\mathrm{b}}$ (Rotamere), $\left.1 \mathrm{H}, \mathrm{NH}\right), 9.84,9.88$ (2 s (Rotamere), $\left.1 \mathrm{H}, 2^{\prime}-\mathrm{H}\right)$.

${ }^{13}$ C-NMR (50.3 MHz, $\mathrm{CDCl}_{3}$ ): $\delta=21.32$ (C-4), 39.31 (C-3), 46.46 (C-1), 49.47 (C1'), 67.59 ( $\mathrm{Ph}-\mathrm{CH}_{2}$ ), 108.7 (C-4a), 111.2 (C-8), 118.2 (C-7), 119.6 (C-5), 122.2 (C-6), 126.5 (C-4b), 127.9 (Ph-C-2", Ph-C-6"'), 128.2 (Ph-C-4'), 128.6 (Ph-C-3", Ph-C5'), 132.5 (C-9a), 136.1 (C-8a), 136.6 (Ph-C-1'), 155.2 (C-2'”), 201.8 (C-2').

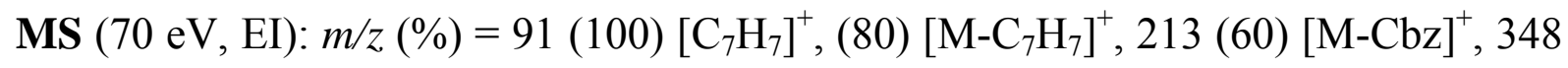
(40) $[\mathrm{M}]^{+}$.

$\mathbf{C}_{21} \mathbf{H}_{20} \mathbf{N}_{2} \mathbf{O}_{3}(348.15)$ 


\section{Synthese des Aldehyds 118}

\subsection{2-Benzyl-9-tert-butyl-1-(2-ethoxy-2-oxoethyl)-3,4-dihydro-1H-pyrido[3,4-}

\section{b]indol-2,9-dicarboxylat (174)}

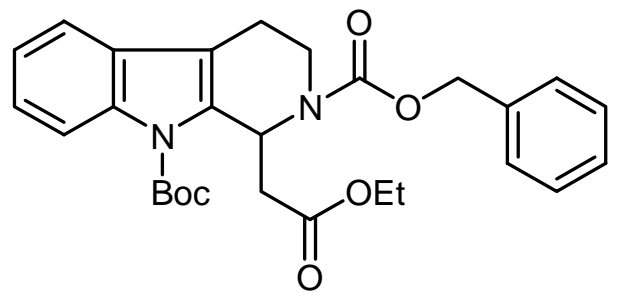

$\mathrm{Zu}$ einer Lösung des Amins 121 (9.6 g, $24.5 \mathrm{mmol}, 1.0$ Äq.) in $\mathrm{CH}_{3} \mathrm{CN}(80 \mathrm{~mL}$, $3.0 \mathrm{~mL}$ pro mmol) wurde eine Lösung von 2,2-Dimethylpropionsäureanhydrid ( $6.8 \mathrm{~g}$, $31.2 \mathrm{mmol}, 1.27$ Äq.) in $\mathrm{CH}_{3} \mathrm{CN}(80 \mathrm{~mL}, 3.0 \mathrm{~mL}$ pro mmol Anhydrid) und DMAP (826 mg, $6.8 \mathrm{mmol}, 0.28$ Äq.) bei Raumtemperatur dazugegeben und die resultierende Reaktionsmischung wurde $12 \mathrm{~h}$ bei der selben Temperatur gerührt. Anschließend wurde mit $0.4 \mathrm{~N} \mathrm{HCl}(160 \mathrm{~mL})$ gequentscht und die organische Phase mit $\mathrm{CH}_{2} \mathrm{Cl}_{2}(2 \mathrm{x}$ $160 \mathrm{~mL}$ ) extrahiert. Die vereinigten organischen Phasen wurden mit gesättigter $\mathrm{NaHCO}_{3^{-}}$und $\mathrm{NaCl}$-Lösung gewaschen, über $\mathrm{Na}_{2} \mathrm{SO}_{4}$ getrocknet und das Lösungsmittel im Vakuum entfernt. Man erhielt die Titelverbindung (11.2 g, 22.7 mmol, 93 \%) als ein hellbraunes Öl.

$\mathbf{R}_{\mathbf{f}}=0.41(\mathrm{EE} / \mathrm{P}=1: 4)$.

UV $\left(\mathrm{CH}_{3} \mathrm{CN}\right): \lambda_{\max }(\lg \varepsilon)=228.5$ (0.557), 265.5 (0.336), 293.0 (0.093).

IR $(\mathrm{KBr}): v=2980 \mathrm{~cm}^{-1}(\mathrm{CH}), 1732(\mathrm{C}=\mathrm{O}), 1457\left(\mathrm{CH}_{2}\right), 1424,1323\left(\mathrm{CH}_{3}\right), 1141(\mathrm{C}-$ O), 1116, $1036\left(\mathrm{OCH}_{2}\right), 855,749$ (1,2-disubst. Benzol), 699 (monosubst. Benzol).

${ }^{1}$ H-NMR $\left(300 \mathrm{MHz}, \mathrm{CDCl}_{3}\right): \delta=1.16\left(\mathrm{~m}, 3 \mathrm{H}, \mathrm{CH}_{3}\right), 1.60,173(2 \mathrm{~s}$ (Rotameres), $9 \mathrm{H}$, $\left.\mathrm{C}\left(\mathrm{CH}_{3}\right)_{3}\right), 2.60-3.10\left(\mathrm{~m}, 4 \mathrm{H}, 4-\mathrm{H}_{2}, 1\right.$ '- $\left.\mathrm{H}_{2}\right), 3.32\left(\mathrm{~m}_{\mathrm{c}}, 1 \mathrm{H}, 3-\mathrm{H}_{\mathrm{a}}\right), 3.99-4.15(\mathrm{~m}, 2 \mathrm{H}$, $\mathrm{CH}_{2}$ ), 4.40 (dd (Rotamere), 0.6 H, 3- $\mathrm{H}_{\mathrm{b}}, J=6.0,14.4 \mathrm{~Hz}$ ), 4.52 (m (Rotamere), $0.4 \mathrm{H}$, 3- $\mathrm{H}_{\mathrm{b}}$ ), 5.14, 5.18 (2 s (Rotameres), $\left.2 \mathrm{H}, \mathrm{Ph}-\mathrm{CH}_{2}\right), 6.27,6.37$ ( $2 \mathrm{dd}, 1 \mathrm{H}, 1-\mathrm{H}, J=3.5$, $10.2 \mathrm{~Hz}$ ), 7.18-7.44 (m, 8 H, 7-H, 8-H, 9-H, Ph-H), 8.18 (d, 1 H, 10-H, J = 7.0 Hz).

${ }^{13}$ C-NMR (50.3 MHz, $\left.\mathrm{CDCl}_{3}\right): \delta=14.11\left(\mathrm{CH}_{3}\right), 20.59,21.26(\mathrm{C}-4), 28.08,28.21$ $\left(\mathrm{C}\left(\mathrm{CH}_{3}\right)_{3}\right), 36.35,36.80(\mathrm{C}-3), 38.96,39.10\left(\mathrm{C}-1\right.$ ') $, 49.86(\mathrm{C}-1), 60.66\left(\mathrm{CH}_{2}\right), 67.34$, 
$67.75\left(\mathrm{Ph}-\mathrm{CH}_{2}\right), 84.77\left(\mathrm{C}\left(\mathrm{CH}_{3}\right)_{3}\right), 115.38(\mathrm{C}-5), 115.86(\mathrm{C}-10), 117.99,118.10(\mathrm{C}-9)$, 122.87 (C-7), 124.64 (C-8), 124.74, 128.03, 128.23 (Ph-C-2'”, 6”, 4”'), 128.44 (Ph-C3",5"'), 128.63 (C-6), 133.53 (C-11), 136.30 (C-11), 136.68 (C-13), 136.68 (Ph-C1'), $149.76(\mathrm{~N}-12-\mathrm{C}=\mathrm{O}), 155.45(\mathrm{~N}-2-\mathrm{C}=\mathrm{O}), 169.91,170.15$ (C-2').

MS $\left(\mathrm{DCI}-\mathrm{NH}_{3}\right): m / z(\%)=510(74)[\mathrm{M}+18]^{+}, 493(38)[\mathrm{M}+1]^{+}$.

$\mathbf{C}_{28} \mathbf{H}_{32} \mathbf{N}_{2} \mathbf{O}_{6}$ (492.23)

\subsection{2-Benzyl-9-tert-butyl-1-(2-oxoethyl)-3,4-dihydro-1H-pyrido[3,4-b]indol-2,9-} dicarboxylat (118)<smiles>O=CCC1c2c(c3ccccc3n2C(=O)c2ccccc2)CCN1C(=O)OCc1ccccc1</smiles>

Analog AAV 2 setzte man den Ester 174 (11.2 g, 22.7 mmol, 1.0 Äq.) mit DIBAH (27.2 mL, $27.2 \mathrm{mmol}, 1.2 \mathrm{Äq} ., 1 \mathrm{M}$ in Hexan) bei $-78{ }^{\circ} \mathrm{C}$ in $\mathrm{CH}_{2} \mathrm{Cl}_{2}(230 \mathrm{~mL})$ innerhalb von $2 \mathrm{~h}$ um. Die Reinigung erfolgte durch Säulenchromatographie (EE/P). Man erhielt die Titelverbindung (5.07 g, $11.3 \mathrm{mmol}, 50 \%)$ als einen farblosen Schaum.

$\mathbf{R}_{\mathbf{f}}=0.32(\mathrm{EE} / \mathrm{P}=1: 2)$.

UV $\left(\mathrm{CH}_{3} \mathrm{CN}\right): \lambda_{\max }(\lg \varepsilon)=228.5(0.621), 264.5$ (0.361), 293.0 (0.102).

IR (KBr): $v=2979 \mathrm{~cm}^{-1}(\mathrm{CH}), 1725(\mathrm{C}=\mathrm{O}), 1456\left(\mathrm{CH}_{2}\right), 1423,1369\left(\mathrm{CH}_{3}\right), 1235(\mathrm{O}-$ Ar), 1143 (C-O), 1117, $1016\left(\mathrm{OCH}_{2}\right), 853,751$ (1,2-disubst. Benzol), 699 (monosubst. Benzol).

${ }^{1} \mathbf{H}-\mathrm{NMR}\left(300 \mathrm{MHz}, \mathrm{CDCl}_{3}\right): \delta=1.58,1.69\left(2 \mathrm{~s}\right.$ (Rotamere), $\left.9 \mathrm{H}, \mathrm{C}\left(\mathrm{CH}_{3}\right)_{3}\right), 2.50-3.30$ (m, $5 \mathrm{H}, 3-\mathrm{H}_{\mathrm{a}}, 4-\mathrm{H}_{2}, 1^{\prime}-\mathrm{H}_{2}$ ), 4.37, 4.51 (2 dd (Rotamere), $1 \mathrm{H}, 3-\mathrm{H}_{\mathrm{b}}, J=5.0,13.8 \mathrm{~Hz}$ ), 5.16 (dd, $2 \mathrm{H}, \mathrm{Ph}-\mathrm{CH}_{2}, J=15.0 \mathrm{~Hz}$ ), 6.38, 6.53 (2 d (Rotamere), $1 \mathrm{H}, 1-\mathrm{H}, J=8.9 \mathrm{~Hz}$ ), 
7.17-7.38 (m, 8 H, 7-H, 8-H, 9-H, Ph-H), 8.07 (d, 1 H, 10-H, J = 7.5 Hz), 9.77, 9.90 (2 d (Rotamere), $1 \mathrm{H}, \mathrm{CHO}, J=4.0 \mathrm{~Hz}$ ).

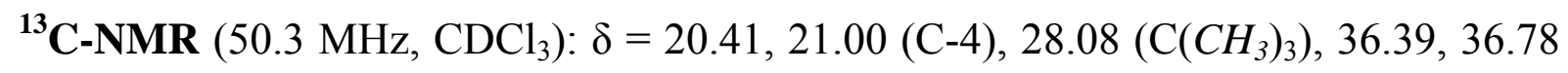
(C-3), 47.67 (C-1'), $\left.48.16(\mathrm{C}-1), 67.46,67.76\left(\mathrm{Ph}^{-} \mathrm{CH}_{2}\right), 84.60\left(\mathrm{C}_{(\mathrm{CH}}\right)_{3}\right), 115.43$, 115.97 (C-5), 115.79 (C-10), 117.94, 118.03 (C-9), 122.92 (C-7), 124.51, 124.60 (C8), 127.73, 128.03, 128.29 (Ph-C-2', 4', 6'), 128.41 (Ph-C-3', 5'), 128.49 (C-6) 133.51 (C-13), 135.61 (C-11), 136.22 (Ph-C-1”), 149.85 (N-12-C=O), 155.47 (N-2$\mathrm{C}=\mathrm{O}), 200.07,200.86$ (C-2').

MS (DCI-NH $\left.)_{3}\right): m / z(\%)=466(100)[\mathrm{M}+18]^{+}, 914$ (3) $[2 \mathrm{M}+18]^{+}$.

$\mathrm{C}_{26} \mathrm{H}_{28} \mathrm{~N}_{2} \mathrm{O}_{5}(448.20)$ 


\section{Synthese des enantiomerenreinen Esters $S-123$}

\subsection{Ethyl-2-(4,9-dihydro-3H-pyrido[3,4-b]indol-1-yl)acetate (124)}<smiles>CCOC(=O)CC1=NCCc2c1[nH]c1ccccc21</smiles>

Analog AAV 4 setzte man das Amin 123 (10.0 g, 38.8 mmol, 1.0 Äq.) mit $\mathrm{KMnO}_{4}$ (38.6 g, $244 \mathrm{mmol}, 6.3$ Äq.) bei $0{ }^{\circ} \mathrm{C}$ in THF ( $350 \mathrm{~mL}$ ) innerhalb von $2 \mathrm{~h} \mathrm{um}$. Man erhielt die Titelverbindung (7.68 g, $30.0 \mathrm{mmol}, 77$ \%) als ein braun-violetter Feststoff.

$\mathbf{R}_{\mathbf{f}}=0.75$ (EE).

MS $\left(\right.$ DCI-NH $\left.{ }_{3}\right): m / z(\%)=257(100)[\mathrm{M}+1]^{+}$.

$\mathbf{C}_{15} \mathbf{H}_{16} \mathbf{N}_{2} \mathbf{O}_{2}(256.12)$

6.2. (S)-Ethyl-2-(2,3,4,9-tetrahydro-1H-pyrido[3,4-b]indol-1-yl)acetate (123)<smiles>CCOC(=O)C[C@H]1NCCc2c1[nH]c1ccccc21</smiles>

Analog AAV 7 setzte man das Imin 124 (50 mg, $195 \mu$ mol, 1.0 Äq.) mit dem Katalysator 125 (5.7 mg, 19.5 mol, 0.1 Äq.) und einer Mischung von Ameisensäure / $\mathrm{Net}_{3}(5: 2,0.1 \mathrm{~mL})$ bei Raumtemperatur in $\mathrm{CH}_{3} \mathrm{CN}(0.4 \mathrm{~mL})$ für $42 \mathrm{~h}$ um. Die Reinigung erfolgte durch Säulenchromatographie (EE/P). Man erhielt die Titelverbindung (46 mg, $177 \mu \mathrm{mol}, 91 \%$ ) als dunkelbraunes Öl.

$[\alpha]_{\mathbf{D}}^{20}=+23^{\circ}\left(c=0.2, \mathrm{CHCl}_{3}\right)$. 


\section{Synthese der Imine 167 und 168}

\subsection{2-(2,3,4,9-Tetrahydro-1H-pyrido[3,4-b]indol-1-yl)ethanol (164)}<smiles>OCCC1NCCc2c1[nH]c1ccccc21</smiles>

Analog AAV 3 setzte man den Ester 123 (500 mg, 1.94 mmol, 1.0 Äq.) mit $\mathrm{LiAlH}_{4}$ (220 mg, $5.81 \mathrm{mmol}, 3.0$ Äq.) in THF (30.0 mL) innerhalb von $2 \mathrm{~h}$ um. Die Reinigung erfolgte durch Säulenchromatographie (EE/P). Man erhielt die Titelverbindung (344 mg, $1.61 \mathrm{mmol}, 83$ \%) als einen weißen Schaum.

$\mathbf{R}_{\mathbf{f}}=0.53(\mathrm{EE})$.

UV $\left(\mathrm{CH}_{3} \mathrm{CN}\right): \lambda_{\max }(\lg \varepsilon)=202.5$ (0.910), 224.5 (1.690), $281.0(0.368), 289.5$ (0.298).

IR (KBr): $v=3288 \mathrm{~cm}^{-1}(\mathrm{NH}, \mathrm{OH}), 2925(\mathrm{CH}), 1452\left(\mathrm{CH}_{2}\right), 1301,1157,1112,1063$, 739 (Phenyl-H).

${ }^{1} \mathbf{H}-\mathbf{N M R}\left(300 \mathrm{MHz}, \mathrm{CDCl}_{3}\right): \delta=1.91$ (q, $\left.2 \mathrm{H}, 1-\mathrm{H}_{2}, J=6.0 \mathrm{~Hz}\right), 2.70\left(\mathrm{~m}_{\mathrm{c}}, 2 \mathrm{H}, 4\right.$ '$\left.\mathrm{H}_{2}\right), 3.00\left(\mathrm{~m}_{\mathrm{c}}, 1 \mathrm{H}, 3^{\prime}-\mathrm{H}_{\mathrm{a}}\right), 3.20\left(\mathrm{dt}, 1 \mathrm{H}, 3^{\prime}-\mathrm{H}_{\mathrm{b}}, J=6.5,15.5 \mathrm{~Hz}\right), 3.48\left(\mathrm{~s}_{\mathrm{b}}, 2 \mathrm{H}, \mathrm{NH}\right.$, $\mathrm{OH}$ ), $3.80\left(\mathrm{~m}_{\mathrm{c}}, 2 \mathrm{H}, 2-\mathrm{H}_{2}\right), 4.19(\mathrm{t}, 1 \mathrm{H}, 1$ '- $\mathrm{H}, J=7.2 \mathrm{~Hz}$ ), 7.10 (quind, $2 \mathrm{H}, 2 \mathrm{x}$ Ar-H, $J=1.5,9.0 \mathrm{~Hz}), 7.24-7.28(\mathrm{~m}, 1 \mathrm{H}, \mathrm{Ar}-\mathrm{H}), 7.44-7.48$ (m, $1 \mathrm{H}, \mathrm{Ar}-\mathrm{H}), 8.56$ (s, $1 \mathrm{H}$, Indol-H).

${ }^{13}$ C-NMR (50.3 MHz, $\mathrm{CDCl}_{3}$ ): $\delta=22.49$ (C-4'), 38.17 (C-1), 42.82 (C-3’), 52.70 (C1'), 62.33 (C-2), 108.01 (C-5'), 110.75 (C-10'), 118.04 (C-7'), 119.01 (C-9'), 121.25 (C-8'), 127.55 (C-6'), 135.45 (C-12'), 136.44 (C-11').

MS $(70 \mathrm{eV}, \mathrm{EI}): m / z(\%)=43(100)\left[\mathrm{C}-\mathrm{CH}_{2}-\mathrm{OH}\right]^{+}, 171(17)\left[\mathrm{M}-\mathrm{CH}_{2}-\mathrm{CH}_{2}-\mathrm{OH}\right]^{+}, 216$ (3) $[\mathrm{M}]^{+}$.

\section{$\mathbf{C}_{13} \mathbf{H}_{16} \mathbf{N}_{2} \mathbf{O}(216.13)$}




\subsection{1-(2-(Triisopropylsilyloxy)ethyl)-2,3,4,9-tetrahydro-1H-pyrido[3,4-b]indol} (166)

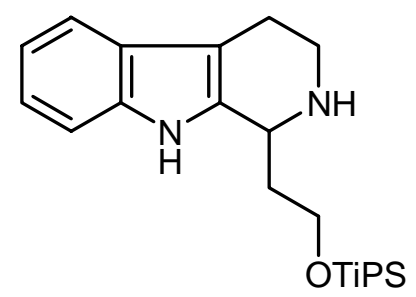

Analog AAV 1 setzte man den Alkohol 164 (170 mg, 785 mol, 1.0 Äq.) mit TiPSCl (0.2 mL, $940 \mu \mathrm{mol}, 1.2$ Äq.), Imidazol (64 mg, $940 \mu \mathrm{mol}, 1.2$ Äq.) und DMAP (9.5 mg, $79 \mu \mathrm{mol}, 0.1$ Äq.) in DMF (2.5 mL) innerhalb von $12 \mathrm{~h} \mathrm{um.} \mathrm{Die} \mathrm{Reinigung}$ erfolgte durch Säulenchromatographie (EE/P). Man erhielt die Titelverbindung (212 mg, $642 \mu \mathrm{mol}, 82 \%$ ) als gelben Feststoff.

$\mathbf{R}_{\mathbf{f}}=0.62(\mathrm{EE} / \mathrm{P}=1: 1)$.

UV $\left(\mathrm{CH}_{3} \mathrm{CN}\right): \lambda_{\max }(\lg \varepsilon)=193.5$ (0.802), 227.5 (1.047), $281.5(0.240)$.

IR (KBr): $v=3395 \mathrm{~cm}^{-1}, 2936,1657,1452,1264,1210,1111,938,741$.

${ }^{1}$ H-NMR (300 MHz, $\left.\mathrm{CDCl}_{3}\right): \delta=1.13\left(\mathrm{dd}, 18 \mathrm{H}, 3 \times \mathrm{CH}\left(\mathrm{CH}_{3}\right)_{2}, J=3.0,9.2 \mathrm{~Hz}\right)$, 1.84-2.12 (m, $\left.3 \mathrm{H}, 3 \times \mathrm{CH}\left(\mathrm{CH}_{3}\right)_{2}\right), 2.75\left(\mathrm{~m}_{\mathrm{c}}, 2 \mathrm{H}, 4-\mathrm{H}_{2}\right), 3.07$ (ddd, $1 \mathrm{H}, 3-\mathrm{H}_{\mathrm{a}}, J=8.5$, 12.1, 19.3 Hz), 3.33 (dt, $1 \mathrm{H}, 3-\mathrm{H}_{\mathrm{b}}, J=8.5,19.3 \mathrm{~Hz}$ ), 3.98 (t, $2 \mathrm{H}, 1^{\prime}-\mathrm{H}_{2}, J=6.9 \mathrm{~Hz}$ ), $4.22\left(\mathrm{~m}_{\mathrm{c}}, 1 \mathrm{H}, 1-\mathrm{H}\right)$, 7.04-7.30 (m, $3 \mathrm{H}$, Indol-H), 7.50 (d, $\left.1 \mathrm{H}, J=11.0 \mathrm{~Hz}\right), 9.17$ ( $\mathrm{s}_{\mathrm{b}}, 1$ $\mathrm{H}$, Indol-NH).

${ }^{13} \mathrm{C}-\mathrm{NMR}\left(50.3 \mathrm{MHz}, \mathrm{CDCl}_{3}\right): \delta=11.74\left(3 \times \mathrm{CH}\left(\mathrm{CH}_{3}\right)_{2}\right), 17.86\left(3 \times \mathrm{CH}\left(\mathrm{CH}_{3}\right)_{2}\right)$, 22.49 (C-4), 38.17 (C-1'), 42.82 (C-3), 52.70 (C-1), 62.33 C-2'), 108.01 C-8), 110.75 C-5), 118.04 (C-6), 119.01 (C-7), 121.25 (C-4a), 127.55 (C-4b), 135.45 (C-9a), 136.44 (C-8a).

MS $\left(\mathrm{DCI}-\mathrm{NH}_{3}\right): m / z(\%)=373(100)[\mathrm{M}+1]^{+}, 746(4)[2 \mathrm{M}+1]^{+}$.

$\mathrm{C}_{22} \mathbf{H}_{36} \mathbf{N}_{2} \mathbf{O S i}$ (372.26) 


\subsection{1-(2-(tert-Butyldimethylsilyloxy)ethyl)-2,3,4,9-tetrahydro-1H-pyrido[3,4-}

\section{b]indol (165)}

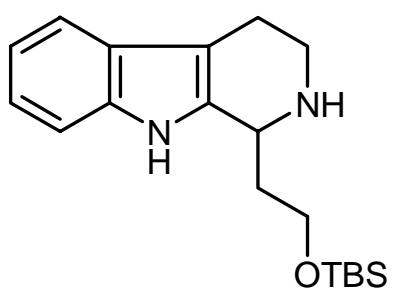

Analog AAV 1 setzte man den Alkohol 164 (315 mg, 1.46 mmol, 1.0 Äq.) mit TBSCl (264 mg, 1.75 mmol, 1.2 Äq.), Imidazol (119 mg, 1.75 mmol, 1.2 Äq.) und DMAP (18.0 mg, 146 mol, 0.1 Äq.) in DMF (5.0 mL) innerhalb von $12 \mathrm{~h}$ um. Die Reinigung erfolgte durch Säulenchromatographie (EE/P). Man erhielt die Titelverbindung (242 mg, $733 \mu \mathrm{mol}, 50 \%$ ) als weißen Feststoff.

$\mathbf{R}_{\mathbf{f}}=0.64(\mathrm{EE} / \mathrm{P}=1: 1)$.

UV $\left(\mathrm{CH}_{3} \mathrm{CN}\right): \lambda_{\max }(\lg \varepsilon)=202.0(0.695), 224.5(1.177), 281.5(0.261), 289.5(0.214)$.

IR (KBr): $v=3395 \mathrm{~cm}^{-1} 2927,1455,1300,1255,1116,1077,1028,1009,966,838$, 777,735 .

${ }^{1} \mathrm{H}-\mathrm{NMR}\left(300 \mathrm{MHz}, \mathrm{CDCl}_{3}\right): \delta=0.05,0.10\left(2 \mathrm{x} \mathrm{s}, 6 \mathrm{H}, 2 \times \mathrm{CH}_{3}\right), 0.92(\mathrm{~s}, 9 \mathrm{H}$, $\left.\mathrm{C}\left(\mathrm{CH}_{3}\right)_{3}\right), 1.93-2.03$ (m, $1 \mathrm{H}, 1$ '- $\left.\mathrm{Ha}\right), 2.13-2.21$ (m, $1 \mathrm{H}, 1$ '- $\left.\mathrm{Hb}\right), 2.62-2.81$ (m, $3 \mathrm{H}, 3-$ Ha, 4- $\mathrm{H}_{2}$ ), 2.95 (ddd, $1 \mathrm{H}, 3-\mathrm{Hb}, J=8.0,12.2,18.5 \mathrm{~Hz}$ ), 3.24-3.36 (m, $\left.1 \mathrm{H}, 1-\mathrm{H}\right), 3.83$ $\left(\mathrm{m}_{\mathrm{c}}, 2 \mathrm{H}, 2^{\prime}-\mathrm{H}_{2}\right), 6.81-7.09$ (m, $2 \mathrm{H}$, Indol-H), 7.27 (d, $1 \mathrm{H}$, Indol-H, $\left.J=9.3 \mathrm{~Hz}\right), 7.38$ (d, $1 \mathrm{H}$, Indol-H, $J=9.3 \mathrm{~Hz}$ ).

${ }^{13}$ C-NMR $\left(50.3 \mathrm{MHz}, \mathrm{CDCl}_{3}\right): \delta=-5.36\left(2 \times \mathrm{CH}_{3}\right), 19.06\left(\mathrm{C}\left(\mathrm{CH}_{3}\right)_{3}\right), 22.96\left(\mathrm{C}\left(\mathrm{CH}_{3}\right)_{3}\right)$, 26.40 (C-4), 37.18 (C-1'), 43.37 (C-3), 52.96 (C-1), 62.24 (C-2'), 108.99 (C-6), 111.78 (C-8), 118.55 (C-5), 119.64 (C-7), 121.98 (C-4a), 128.63 (C-4b), 136.19 (C9a), 137.65 (C-8a).

MS $\left(\mathrm{DCI}-\mathrm{NH}_{3}\right): m / z(\%)=331(100)[\mathrm{M}+1]^{+}, 348(24)[\mathrm{M}+18]^{+}, 661(6)[2 \mathrm{M}+1]^{+}$.

$\mathrm{C}_{19} \mathrm{H}_{30} \mathrm{~N}_{2} \mathrm{OSi}(330.21)$ 


\subsection{1-(2-(Triisopropylsilyloxy)ethyl)-4,9-dihydro-3H-pyrido[3,4-b]indol (167)}<smiles>Sc1ccccc1CCC1=NCCc2c1[nH]c1ccccc21</smiles>

Analog AAV 4 setzte man das Amin 166 (473 mg, 1.27 mmol, 1.0 Äq.) mit $\mathrm{KMnO}_{4}$ (422 mg, $2.67 \mathrm{mmol}, 2.1$ Äq.) bei $0{ }^{\circ} \mathrm{C}$ in $\mathrm{CH}_{3} \mathrm{CN}(12.0 \mathrm{~mL})$ und $\mathrm{MeOH}(2.0 \mathrm{~mL})$ innerhalb von $1 \mathrm{~h}$ bei $0{ }^{\circ} \mathrm{C}$ um. Man erhielt die Titelverbindung $(362 \mathrm{mg}, 0.98 \mathrm{mmol}$, $77 \%$ ) als einen braun-violetten Feststoff.

$\mathbf{R}_{\mathbf{f}}=0.78(\mathrm{EE})$.

MS $\left.(\text { DCI-NH })_{3}\right): m / z(\%)=371(100)[\mathrm{M}+1]^{+}, 741(32)[2 \mathrm{M}+1]^{+}$.

$\mathrm{C}_{22} \mathbf{H}_{34} \mathbf{N}_{2} \mathbf{O S i}(370.24)$

\subsection{1-(2-(tert-Butyldimethylsilyloxy)ethyl)-4,9-dihydro-3H-pyrido[3,4-b]indol} (168)<smiles>CCOCCC1=NCCc2c1[nH]c1ccccc21</smiles>

Analog AAV 4 setzte man das Amin 165 (222 mg, 0.67 mmol, 1.0 Äq.) mit $\mathrm{KMnO}_{4}$ (221 mg, $1.40 \mathrm{mmol}, 2.1 \mathrm{Äq}$.) bei $0^{\circ} \mathrm{C}$ in $\mathrm{CH}_{3} \mathrm{CN}(6.0 \mathrm{~mL})$ und $\mathrm{MeOH}(1.0 \mathrm{~mL})$ innerhalb von $1 \mathrm{~h}$ bei $0{ }^{\circ} \mathrm{C}$ um. Man erhielt die Titelverbindung (176 mg, $0.54 \mathrm{mmol}$, $80 \%$ ) als einen braun-violetten Feststoff.

$\mathbf{R}_{\mathbf{f}}=0.80(\mathrm{EE})$. 
MS $\left(\mathrm{DCI}-\mathrm{NH}_{3}\right): m / z(\%)=329(100)[\mathrm{M}+1]^{+}, 676(42)[2 \mathrm{M}+18]^{+}$.

$\mathrm{C}_{19} \mathrm{H}_{28} \mathbf{N}_{2}$ OSi (328.20) 


\section{Synthese des Modelsystems 172 zur Überprüfung der Noyori-}

\section{Reduktion}

\section{1. $\quad N$-(3,4-Dimethoxyphenyl)ethylacetamid (170)}

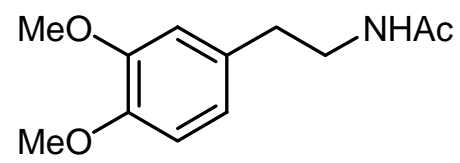

Eine Lösung von 2-(3,4-Dimethoxyphenyl)ethylamin (4.5 g, $24.8 \mathrm{mmol}, 1.0$ Äq.) und DMAP (303 mg, $2.48 \mathrm{mmol}, 0.1$ Äq.) in $\mathrm{CH}_{2} \mathrm{Cl}_{2}(25.0 \mathrm{~mL}, 1.0 \mathrm{~mL}$ pro mmol Amin) wurde bei $0^{\circ} \mathrm{C}$ mit $\mathrm{NEt}_{3}(12.5 \mathrm{~mL})$ versetzt. Acetanhydrid (2.5 mL) wurde tropfenweise bei derselben Temperatur zugegeben und das Reaktionsgemisch für $24 \mathrm{~h}$ bei Raumtemperatur gerührt. Die organische Phase wurde mit $\mathrm{H}_{2} \mathrm{O}(200 \mathrm{~mL}), 2 \mathrm{~N} \mathrm{HCl}$ (100 mL), gesättigter $\mathrm{NaHCO}_{3}-(2$ x $200 \mathrm{~mL})$ und NaCl-Lösung $(200 \mathrm{~mL})$ gewaschen und über $\mathrm{MgSO}_{4}$ getrocknet. Das Lösungsmittel wurde im Vakuum entfernt und das Rohprodukt umkristallisiert (EE/P). Man erhielt die Titelverbindung (4.74 g, 21.2 mmol, $86 \%$ ) als farblose Kristalle.

$\mathbf{R}_{\mathbf{f}}=0.71\left(\mathrm{CH}_{2} \mathrm{Cl}_{2}: \mathrm{MeOH} ; 7: 1\right)$.

Smp. $=94{ }^{\circ} \mathrm{C}$.

UV $\left(\mathrm{CH}_{3} \mathrm{CN}\right): \lambda_{\max }(\lg \varepsilon): 201.5 \mathrm{~nm}$ (2.611), 230.0 (0.468), 280.0 (0.164).

IR (KBr): $v=3254 \mathrm{~cm}^{-1}(\mathrm{NH}), 1634$ ( $N, N$-disubst. Amid), 1518 (C=C-Aromat), 1263 (O-Ar), 1156, 1139, $1020\left(\mathrm{O}-\mathrm{CH}_{3}\right) 815$ (aromat. H), 767, 611.

${ }^{1} \mathbf{H}-\mathbf{N M R}\left(300 \mathrm{MHz}, \mathrm{CDCl}_{3}\right): \delta=1.90\left(\mathrm{~s}, 3 \mathrm{H}, \mathrm{CH}_{3}\right), 2.76\left(\mathrm{t}, 2 \mathrm{H}, 1-\mathrm{H}_{2}, J=7.0 \mathrm{~Hz}\right)$, $3.48\left(\mathrm{dt}, 2 \mathrm{H}, 2-\mathrm{H}_{2}, J=6.0,7.0 \mathrm{~Hz}\right), 3.85\left(\mathrm{~s}, 3 \mathrm{H}, \mathrm{OCH}_{3}\right), 3.86\left(\mathrm{~s}, 3 \mathrm{H}, \mathrm{OCH}_{3}\right), 5.93$ $\left(\mathrm{s}_{\mathrm{b}}, 1 \mathrm{H}, \mathrm{NH}\right), 6.71-6.82(\mathrm{~m}, 3 \mathrm{H}$, Aryl-H).

${ }^{13}$ C-NMR $\left(50.3 \mathrm{MHz}, \mathrm{CDCl}_{3}\right): \delta=23.08\left(\mathrm{CH}_{3}\right), 35.01$ (C-1), $40.64(\mathrm{C}-2), 55.65$, $55.71\left(2 \times \mathrm{OCH}_{3}\right), 111.15,111.68,120.42$ (C-2', C-5', C-6'), 131.24 (C-1'), 147.44 (C4'), 148.79 (C-3'), $169.97(\mathrm{C}=\mathrm{O})$.

MS $\left(\mathrm{DCI}-\mathrm{NH}_{3}\right): m / z(\%)=241(100)[\mathrm{M}+18]^{+}, 447(11)[2 \mathrm{M}+1]^{+}, 464(11)[2 \mathrm{M}+18]^{+}$. $\mathrm{C}_{12} \mathrm{H}_{17} \mathrm{NO}_{3}(223.12)$ 


\subsection{6,7-Dimethoxy-1-methyl-3,4-dihydroisochinolin (171)}<smiles>COc1cc2c(cc1OC)C(C)=NCC2</smiles>

Das Amid 170 (6.0 g, $26.9 \mathrm{mmol}, 1.0$ Äq.) wurde in Toluol (30.0 mL, $1.0 \mathrm{~mL}$ pro mmol) gelöst und $\mathrm{POCl}_{3}(5.91 \mathrm{~mL})$ langsam über $15 \mathrm{~min}$ zugegeben. Die Reaktionslösung wurde unter Reflux für $2 \mathrm{~h}$ gerührt und anschließend bei $4{ }^{\circ} \mathrm{C}$ für 12 h, wobei man eine gelbe Suspension erhielt. Der Rückstand wurde abfiltriert und mit kaltem Methanol und Essigester gewaschen. Dieser wurde dann umkristallisiert (MeOH/EE). Man erhielt die Titelverbindung (4.55 g, $22.2 \mathrm{mmol}, 83 \%$ ) als einen weißen Feststoff.

$\mathbf{R}_{\mathbf{f}}=0.69\left(\mathrm{CH}_{2} \mathrm{Cl}_{2} / \mathrm{MeOH}=7: 1\right)$.

Smp. $=202-203^{\circ} \mathrm{C}$.

UV $\left(\mathrm{CH}_{3} \mathrm{CN}\right): \lambda_{\max }(\lg \varepsilon): 231.0 \mathrm{~nm}(0.551), 243.5$ (0.671), $301.5(0.398), 352.0$ (0.309).

IR (KBr): $v=2611 \mathrm{~cm}^{-1}, 1656(\mathrm{C}=\mathrm{N}), 1565(\mathrm{C}=\mathrm{C}-$ Aromat $), 1335,1278(\mathrm{O}-\mathrm{Ar}), 1167$, $1069\left(\mathrm{OCH}_{3}\right)$.

${ }^{1} \mathbf{H}-\mathbf{N M R}\left(300 \mathrm{MHz}, \mathrm{CDCl}_{3}\right): \delta=2.36\left(\mathrm{~s}, 3 \mathrm{H}, \mathrm{CH}_{3}\right), 2.64\left(\mathrm{t}, 2 \mathrm{H}, 4-\mathrm{H}_{2}, J=7.0 \mathrm{~Hz}\right)$, $3.63\left(\mathrm{td}, 2 \mathrm{H}, 3-\mathrm{H}_{2}, J=1.5,7.0 \mathrm{~Hz}\right), 3.88\left(\mathrm{~s}, 3 \mathrm{H}, \mathrm{OCH}_{3}\right), 3.92\left(\mathrm{~s}, 3 \mathrm{H}, \mathrm{OCH}_{3}\right), 6.68(\mathrm{~s}$, $1 \mathrm{H}, 5-H), 6.98(\mathrm{~s}, 1 \mathrm{H}, 8-H)$.

${ }^{13}$ C-NMR $\left(50.3 \mathrm{MHz}, \mathrm{CDCl}_{3}\right): \delta=19.38\left(\mathrm{CH}_{3}\right), 24.89(\mathrm{C}-4), 40.43(\mathrm{C}-3), 56.24$, $56.38\left(2 \times \mathrm{OCH}_{3}\right), 110.63$ (C-8), 111.03 (C-5), 117.71 (C-9), 132.70 (C-10), 148.50 (C-7), 156.09 (C-6), 173.51 (C-1).

MS (70 eV, EI): $m / z(\%)=205(100)[\mathrm{M}]^{+}, 190(48)\left[\mathrm{M}-\mathrm{CH}_{3}\right]^{+}, 174(9)\left[\mathrm{M}-\mathrm{OCH}_{3}\right]^{+}$.

$\mathbf{C}_{12} \mathbf{H}_{15} \mathbf{N O}_{2}(205.11)$ 


\section{3. (S)-6,7-Dimethoxy-1-methyl-1,2,3,4-tetrahydroisochinolin (172)}

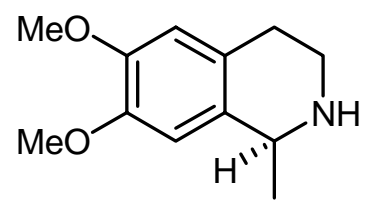

$\mathrm{Zu}$ einer Lösung von dem Imin 171 (1.35 g, $6.60 \mathrm{mmol}, 1.0$ Äq.) und den in situ generierten Katalysator 159 (430 mg, $0.66 \mathrm{mmol}, 1.0$ Äq.) in $\mathrm{CH}_{3} \mathrm{CN}$ (13.2 mL, $2.0 \mathrm{~mL}$ pro mmol Imin) wurde ein azeotropisches Gemisch aus Ameisensäure und $\mathrm{NEt}_{3} \quad(5: 2,3.3 \mathrm{~mL})$ zugegeben. Die Reaktionslösung wurde für $17 \mathrm{~h}$ bei Raumtemperatur gerührt, durch Zugabe von gesättigter $\mathrm{Na}_{2} \mathrm{CO}_{3}$-Lösung auf $\mathrm{pH} 8-9$ gebracht und mit Essigester $(3 \times 20 \mathrm{~mL})$ extrahiert. Die vereinigten organischen Phasen wurden mit gesättigter $\mathrm{NaCl}$-Lösung $(20 \mathrm{~mL})$ gewaschen, über $\mathrm{MgSO}_{4}$ getrocknet und das Lösungsmittel im Vakuum entfernt. Das Rohprodukt wurde durch Säulenchromatographie $\left(\mathrm{EE} / \mathrm{MeOH} / \mathrm{NEt}_{3}\right)$ gereinigt und man erhielt die Titelverbindung (1.11 g, 5.34 mmol, $81 \%)$ als braunes Öl.

$\mathbf{R}_{\mathbf{f}}=0.38\left(\mathrm{CH}_{2} \mathrm{Cl}_{2}: \mathrm{MeOH} ; 7: 1\right)$.

$[\alpha]_{\mathrm{D}}^{20}=10.8^{\circ}\left(c=0.25, \mathrm{CHCl}_{3}\right)$.

UV $\left(\mathrm{CH}_{3} \mathrm{CN}\right): \lambda_{\max }(\lg \varepsilon): 201.0 \mathrm{~nm}$ (1.759), 282.5 (0.186).

IR (KBr): $v=2932 \mathrm{~cm}^{-1}(\mathrm{C}-\mathrm{H}), 1610,1512\left(\mathrm{C}=\mathrm{C}\right.$-Aromat), $1464\left(\mathrm{CH}_{2}\right), 1372\left(\mathrm{CH}_{3}\right)$, 1256, 1126, $1030\left(\mathrm{OCH}_{3}\right), 857,790$.

${ }^{1}$ H-NMR $\left(300 \mathrm{MHz}, \mathrm{CDCl}_{3}\right): \delta=1.42\left(3 \mathrm{H}, \mathrm{CH}_{3}, J=6.7 \mathrm{~Hz}\right), 1.66\left(\mathrm{~s}_{\mathrm{b}}, 1 \mathrm{H}, \mathrm{NH}\right)$, 2.55-2.88 (m, $\left.2 \mathrm{H}, 4-H_{2}\right), 2.99\left(\mathrm{~m}_{\mathrm{c}}, 1 \mathrm{H}, 3-H_{\mathrm{a}}\right), 3.25$ (dt, $\left.1 \mathrm{H}, 3-H_{\mathrm{b}}, J=4.5,12.0 \mathrm{~Hz}\right)$, $3.85\left(\mathrm{~s}, 3 \mathrm{H}, \mathrm{OCH}_{3}\right), 3.86\left(\mathrm{~s}, 3 \mathrm{H}, \mathrm{OCH}_{3}\right), 4.04$ (q, $\left.1 \mathrm{H}, 1-\mathrm{H}, \mathrm{J}=6.7 \mathrm{~Hz}\right), 6.57,6.62$ ( 2 $\mathrm{H}, 6-\mathrm{H}, 7-\mathrm{H})$.

${ }^{13}$ C-NMR (75.5 MHz, $\left.\mathrm{CDCl}_{3}\right): \delta=22.78\left(\mathrm{CH}_{3}\right), 29.48(\mathrm{C}-4), 41.77(\mathrm{C}-3), 51.13(\mathrm{C}-$ 1), 55.73, 55.86 (2 x OMe), 108.89 (C-8), 111.62 (C-5), 126.72 (C-10), 132.39 (C-9), 147.07 (C-6), 147.15 (C-7).

MS $(70 \mathrm{eV}, \mathrm{EI}): m / z(\%)=192(100)\left[\mathrm{M}-\mathrm{CH}_{3}\right]^{+}, 207(10)[\mathrm{M}]^{+}$.

$\mathbf{C}_{12} \mathbf{H}_{17} \mathbf{N O}_{2}(207.13)$ 


\section{Synthese der Acetale}

\subsection{5-(tert-Butyldimethylsilyloxy)pentan-1-ol (132)}<smiles>OCCCCC[OH2+]</smiles>

$\mathrm{Zu}$ einer Lösung von Imidazol (32.0 g, $0.46 \mathrm{~mol}, 1.3$ Äq.) in DMF (350 mL, $0.18 \mathrm{~mL}$ pro mmol Diol) wurde bei Raumtemperatur 1,5-Pentandiol (210 mL, 2.0 mol, 5.6 Äq.) dazugegeben und die Reaktionsmischung auf $0^{\circ} \mathrm{C}$ gekühlt. Anschließend wurde TBSCl (54.0 g, $0.36 \mathrm{~mol}, 1.0$ Äq.) zugetropft und weitere $30 \mathrm{~min}$ bei $0^{\circ} \mathrm{C}$ gerührt, mit Ether $(350 \mathrm{~mL})$ verdünnt und dann mit $\mathrm{H}_{2} \mathrm{O}(350 \mathrm{~mL})$ und gesättigter NaCl-Lösung $(700 \mathrm{~mL})$ gewaschen. Die wässrige Phase wurde mit Ether $(4 \times 700 \mathrm{~mL})$ extrahiert, über $\mathrm{MgSO}_{4}$ getrocknet und das Lösungsmittel im Vakuum entfernt. Der Rückstand wurde fraktioniert destilliert. Man erhielt die Titelverbindung (72.3 g, 0.33 mol, 92 \%) als ein farbloses Öl.

$\mathbf{R}_{\mathbf{f}}=0.55(\mathrm{P} / \mathrm{EE}=1: 1)$.

Smp. $=75-83^{\circ} \mathrm{C}$ bei 0.3 mbar.

UV $\left(\mathrm{CH}_{3} \mathrm{CN}\right): \lambda_{\max }(\lg \varepsilon): 297.5 \mathrm{~nm}(0.007)$.

IR (KBr): $v=3395 \mathrm{~cm}^{-1}, 2933,2859,1651,1472,1388,1255,1102,939,837,776$, 662.

${ }^{1}$ H-NMR $\left(200 \mathrm{MHz}, \mathrm{CDCl}_{3}\right): \delta=0.00\left(\mathrm{~s}, 6 \mathrm{H}, 2\right.$ x $\left.\mathrm{SiCH}_{3}\right), 0.85\left(\mathrm{~s}, 9 \mathrm{H}, \mathrm{C}\left(\mathrm{CH}_{3}\right)_{3}\right)$, 1.28-1.63 (m, $\left.6 \mathrm{H}, 2-\mathrm{H}_{2}, 3-\mathrm{H}_{2}, 4-\mathrm{H}_{2}\right), 3.59$ (q, $4 \mathrm{H}, 1-\mathrm{H}_{2}, 5-\mathrm{H}_{2}, J=6.3 \mathrm{~Hz}$ ).

${ }^{13}$ C-NMR $\left(50.3 \mathrm{MHz}, \mathrm{CDCl}_{3}\right): \delta=-5.3\left(\mathrm{SiCH}_{3}\right), 18.3\left(\mathrm{C}\left(\mathrm{CH}_{3}\right)_{3}\right), 22.0(\mathrm{C}-3), 25.9$ $\left(\mathrm{C}\left(\mathrm{CH}_{3}\right)_{3}\right), 32.4,32.4(\mathrm{C}-2, \mathrm{C}-4), 62.7,63.1$ (C-1, C-5).

MS $\left(\mathrm{DCI}-\mathrm{NH}_{3}\right): m / z(\%)=236(100)[\mathrm{M}+18]^{+}, 219(97)[\mathrm{M}+1]^{+}$.

$\mathrm{C}_{11} \mathbf{H}_{26} \mathbf{O}_{2} \mathrm{Si}$ (218.17) 


\subsection{5-( tert-Butyldimethylsilyloxy)pentanal (133)}

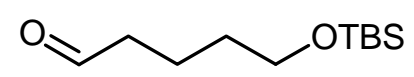

Variante 1:

$\mathrm{Zu}$ einer auf $-78^{\circ} \mathrm{C}$ gekühlten Lösung von DMSO (5.24 mL, 74.0 mmol, 3.2 Äq.) in $\mathrm{CH}_{2} \mathrm{Cl}_{2}\left(70.0 \mathrm{~mL}, 3.0 \mathrm{~mL}\right.$ pro mmol Alkohol) wurde eine auf $-78^{\circ} \mathrm{C}$ vorgekühlte Lösung von Oxalylchlorid (3.21 mL, $36.8 \mathrm{mmol}, 1.6$ Äq.) in $\mathrm{CH}_{2} \mathrm{Cl}_{2}$ (140 mL, $6.0 \mathrm{~mL}$ pro mmol Alkohol) durch eine Transferkanüle getropft. Nachdem 30 min bei $-78^{\circ} \mathrm{C}$ gerührt worden ist, wurde eine Lösung von dem Alkohol 132 (5.0 g, 23.0 mmol, 1.0 Äq.) in $\mathrm{CH}_{2} \mathrm{Cl}_{2}$ (45 mL, $2.0 \mathrm{~mL}$ pro mmol Alkohol) und nach weiteren $90 \mathrm{~min}$ $\mathrm{NEt}_{3}(13.8 \mathrm{~mL}, 0.6 \mathrm{~mL}$ pro mmol Alkohol) zugetropft. Die Reaktionslösung wurde noch weitere $5 \mathrm{~min}$ bei $-78{ }^{\circ} \mathrm{C}$ und dann $30 \mathrm{~min}$ bei Raumtemperatur gerührt. Man gab $\mathrm{H}_{2} \mathrm{O}\left(160 \mathrm{~mL}, 7.0 \mathrm{~mL}\right.$ pro mmol Alkohol) zu und extrahierte mit $\mathrm{CH}_{2} \mathrm{Cl}_{2}(4 \mathrm{x} 160 \mathrm{~mL}$, $7.0 \mathrm{~mL}$ pro mmol Alkohol). Die vereinigten organischen Phasen wurden über $\mathrm{Na}_{2} \mathrm{SO}_{4}$ getrocknet und das Lösungsmittel im Vakuum entfernt. Das Rohprodukt wurde säulenchromatographisch (EE/P) gereinigt. Man erhielt die Titelverbindung (2.8 g, $13.0 \mathrm{mmol}, 57 \%$ ) als leicht gelbliches Öl.

\section{Variante 2:}

$\mathrm{Zu}$ einer Lösung des Alkohols 132 (13.6 g, 62.4 mmol, 1.0 Äq.) in $\mathrm{CH}_{2} \mathrm{Cl}_{2}$ (240 mL, $3.8 \mathrm{~mL}$ pro mmol) wurde bei $0^{\circ} \mathrm{C}$ eine wässrige $1 \mathrm{M} \mathrm{KBr}-L o ̈ s u n g ~(3.12 \mathrm{~mL}$, $3.12 \mathrm{mmol}, 0.05$ Äq.), TEMPO (98 mg, $0.62 \mathrm{mmol}, 10 \mathrm{~mol}-\%$ ) und eine frisch zubereitete Lösung bestehend aus einer 1:1-Mischung aus einer $2.1 \mathrm{M}$ wässrigen NaClO-Lösung (17.8 mL, 37.4 mmol, 0.6 Äq., wässrige Lösung mit $13 \%$ aktivierten Chlor) und gesättigter $\mathrm{NaHCO}_{3}(17.8 \mathrm{~mL})$ zugegeben. Das Reaktionsgemisch wurde $4 \mathrm{~h}$ sehr stark bei $0{ }^{\circ} \mathrm{C}$ gerührt. Anschließend wurden die Phasen getrennt und die wässrige mit dem-selben Volumen an Ether extrahiert, die vereinigten organischen Phasen mit $\mathrm{H}_{2} \mathrm{O}$ und gesättigter $\mathrm{NaCl}$-Lösung gewaschen und über $\mathrm{MgSO}_{4}$ getrocknet. Das Lösungsmittel wurde im Vakuum entfernt und das Rohprodukt 
säulenchromatographisch (EE/P) gereinigt. Man erhielt die Titelverbindung (4.98 g, $32.1 \mathrm{mmol}, 74 \%$ ) als ein leicht gelbliches Öl.

$\mathbf{R}_{\mathbf{f}}=0.70(\mathrm{P} / \mathrm{EE}=3: 1)$.

UV $\left(\mathrm{CH}_{3} \mathrm{CN}\right): \lambda_{\max }(\lg \varepsilon): 226.0 \mathrm{~nm}(0.031)$.

IR (KBr): $v=3395 \mathrm{~cm}^{-1}, 2713,1729,1472,1389,1256,1102,1008,837,776,662$.

${ }^{1}$ H-NMR $\left(200 \mathrm{MHz}, \mathrm{CDCl}_{3}\right): \delta=0.00\left(\mathrm{~s}, 6 \mathrm{H}, 2 \times \mathrm{SiCH}_{3}\right), 0.86\left(\mathrm{~s}, 9 \mathrm{H}, \mathrm{C}\left(\mathrm{CH}_{3}\right)_{3}\right)$, 1.42-1.78 (m, $\left.4 \mathrm{H}, 3-\mathrm{H}_{2}, 4-\mathrm{H}_{2}\right), 2.41$ (dt, $\left.2 \mathrm{H}, 2-\mathrm{H}_{2}, J=1.8,7.3 \mathrm{~Hz}\right), 3.58\left(\mathrm{t}, 2 \mathrm{H}, 5-\mathrm{H}_{2}\right.$, $J=5.6 \mathrm{~Hz}), 9.73(\mathrm{t}, 1 \mathrm{H}, 1-\mathrm{H}, J=1.8 \mathrm{~Hz})$.

${ }^{13} \mathrm{C}-\mathrm{NMR}\left(75.5 \mathrm{MHz}, \mathrm{CDCl}_{3}\right): \delta=-5.3\left(\mathrm{SiCH}_{3}\right), 18.3\left(\mathrm{C}\left(\mathrm{CH}_{3}\right)_{3}\right), 18.6(\mathrm{C}-3), 25.9$ $\left(\mathrm{C}\left(\mathrm{CH}_{3}\right)_{3}\right), 32.1(\mathrm{C}-4), 43.6(\mathrm{C}-2), 62.6(\mathrm{C}-5), 202.7(\mathrm{C}-1)$.

MS $\left(\mathrm{DCI}-\mathrm{NH}_{3}\right): \mathrm{m} / \mathrm{z}(\%)=234(100)[\mathrm{M}+18]^{+}, 450(42)[2 \times \mathrm{M}+18]^{+}$.

$\mathbf{C}_{11} \mathbf{H}_{24} \mathbf{O}_{2} \mathbf{S i}(216.15)$

\section{3. tert-Butyl-(5,5-dimethoxypentyloxy)dimethylsilan (134)}

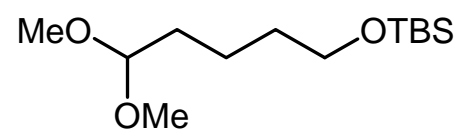

K-10 Montmorellonit-Pulver (77.0 g, $1.38 \mathrm{~g}$ pro mmol Aldehyd) wurde mit $\mathrm{CH}(\mathrm{OMe})_{3}$ (57.8 mL. $1.04 \mathrm{~mL}$ pro mmol Aldehyd) und $\mathrm{MeOH}(57.8 \mathrm{~mL} .1 .04 \mathrm{~mL}$ pro mmol Aldehyd) gemischt. Die resultierende Mischung wurde einige Minuten gerührt und dann abfiltriert. Der nasse Filterkuchen wurde sofort weiterverarbeitet. Der Aldehyd 133 (12.0 g, 55.6 mmol, 1.0 Äq.) wurde mit 2/3 der Menge der Suspension aus K-10 Montmorellonit und $\mathrm{CH}(\mathrm{OMe})_{3}$ in $\mathrm{CH}_{2} \mathrm{Cl}_{2}(240 \mathrm{~mL}, 4.2 \mathrm{~mL}$ pro mmol) für $48 \mathrm{~h}$ bei Raumtemperatur gerührt. Die Suspension wurde über Celite 521 filtriert, hintereinander mit gesättigter $\mathrm{NaHCO}_{3^{-}}, \mathrm{H}_{2} \mathrm{O}$ und $\mathrm{NaCl}-\mathrm{Lösung}$ gewaschen, über $\mathrm{MgSO}_{4}$ getrocknet und das Lösungsmittel im Vakuum entfernt. Das Rohprodukt wurde säulenchromatographisch (EE/P) gereinigt und man erhielt die Titelverbindung (10.9 g, 41.6 mmol, 75 \%) als leicht gelbliches Öl. 
$\mathbf{R}_{\mathbf{f}}=0.64(\mathrm{EE} / \mathrm{P}=1: 4)$.

${ }^{1}$ H-NMR $\left(300 \mathrm{MHz}, \mathrm{CDCl}_{3}\right): \delta=0.02\left(\mathrm{~s}, 6 \mathrm{H}, 2 \times \mathrm{SiCH}_{3}\right), 0.92\left(\mathrm{~s}, 9 \mathrm{~h},\left(\mathrm{C}\left(\mathrm{CH}_{3}\right)_{3}\right)\right.$, 1.29-1.56 (m, $\left.6 \mathrm{H}, 2-\mathrm{H}_{2}, 3-\mathrm{H}_{2}, 4-\mathrm{H}_{2}\right), 3.29\left(\mathrm{~s}, 6 \mathrm{H}, 2\right.$ x $\left.\mathrm{OCH}_{3}\right), 3.79\left(\mathrm{t}, 2 \mathrm{H}, 1-\mathrm{H}_{2}, J=\right.$ $6.8 \mathrm{~Hz}), 4.22(\mathrm{t}, 1 \mathrm{H}, 5-\mathrm{H}, J=6.2 \mathrm{~Hz})$.

${ }^{13}$ C-NMR $\left(50.3 \mathrm{MHz}, \mathrm{CDCl}_{3}\right): \delta=-5.72\left(\mathrm{SiCH}_{3}\right), 16.08(\mathrm{C}-3), 18.16\left(\mathrm{SiC}\left(\mathrm{CH}_{3}\right)_{3}\right)$, $25.81\left(\mathrm{SiC}\left(\mathrm{CH}_{3}\right)_{3}\right), 33.54(\mathrm{C}-2), 33.87(\mathrm{C}-4), 54.53\left(\mathrm{OCH}_{3}\right), 103.93(\mathrm{C}-5)$.

$\mathbf{C}_{14} \mathbf{H}_{32} \mathbf{O}_{3} \mathbf{S i}(276.21)$

\section{4. (5-(Benzyloxy)pentyloxy)-(tert-butyl)dimethylsilan (137)}

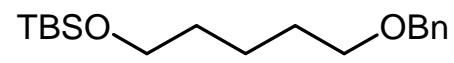

$\mathrm{Zu}$ einer Lösung des Alkohols 132 (4.36.g, 20.0 mmol, 1.0 Äq.) in THF (20.0 mL, $1.0 \mathrm{~mL}$ pro mmol) wurde bei Raumtemperatur $\mathrm{NaH}(0.96 \mathrm{~g}, 24.0 \mathrm{mmol}, 1.2 \mathrm{Äq}$, 60 \%-ig in Weißöl) und Benzylbromid (4.10 g, 24.0 mmol, 1.2 Äq.) zugegeben. Die Reaktionslösung wurde für $12 \mathrm{~h}$ bei Raumtemperatur gerührt, mit der doppelten Menge Ether $(40.0 \mathrm{~mL})$ verdünnt und die organische Phase mit $\mathrm{H}_{2} \mathrm{O}(40 \mathrm{~mL})$ und gesättigter $\mathrm{NaCl}$-Lösung $(40 \mathrm{~mL})$ gewaschen. Es wurde über $\mathrm{Na}_{2} \mathrm{SO}_{4}$ getrocknet, das Lösungsmittel im Vakuum entfernt und das Rohprodukt säulenchromatographisch (EE/P) gereinigt. Man erhielt die Titelverbindung (3.74 g, $12.1 \mathrm{mmol}, 60 \%$ ) als ein farbloses Öl.

$\mathbf{R}_{\mathbf{f}}=0.59(\mathrm{EE} / \mathrm{P}=1: 24)$.

${ }^{1}$ H-NMR $\left(300 \mathrm{MHz}, \mathrm{CDCl}_{3}\right): \delta=-0.01\left(\mathrm{~s}, 6 \mathrm{H}, 2\right.$ x $\left.\mathrm{CH}_{3}\right), 0.85\left(\mathrm{~s}, 9 \mathrm{H}, \mathrm{C}\left(\mathrm{CH}_{3}\right)_{3}\right), 1.16-$ $1.68\left(\mathrm{~m}, 6 \mathrm{H}, 2-\mathrm{H}_{2}, 3-\mathrm{H}_{2}, 4-\mathrm{H}_{2}\right), 3.43\left(\mathrm{t}, 2 \mathrm{H}, 5-\mathrm{H}_{2}, J=9.5 \mathrm{~Hz}\right), 3.56\left(\mathrm{t}, 2 \mathrm{H}, 1-\mathrm{H}_{2}, J=\right.$

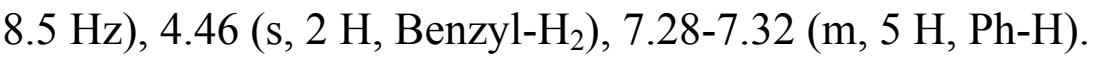

${ }^{13}$ C-NMR (50.3 MHz, $\left.\mathrm{CDCl}_{3}\right): \delta=-5.82\left(\mathrm{SiCH}_{3}\right), 18.14\left(\mathrm{SiC}\left(\mathrm{CH}_{3}\right)_{3}\right), 21.94(\mathrm{C}-3)$, $25.68\left(\mathrm{SiC}\left(\mathrm{CH}_{3}\right)_{3}\right), 30.01(\mathrm{C}-4), 33.29(\mathrm{C}-2), 65.28(\mathrm{C}-1), 72.02$ (C-5), $74.93(\mathrm{Ph}-$ $\left.\mathrm{CH}_{2}\right), 127.42,127.98,128.75,137.50(\mathrm{Ph}-\mathrm{C})$.

$\mathrm{C}_{18} \mathbf{H}_{32} \mathbf{O}_{2} \mathrm{Si}(308.22)$ 


\subsection{5-(Benzyloxy)pentan-1-ol (136)}

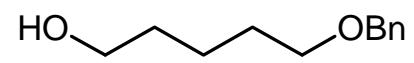

Variante 1:

$\mathrm{Zu}$ einer Lösung des Silylethers 137 (3.6 g, 11.8 mmol, 1.0 Äq.) in THF (120 mL, $10.0 \mathrm{~mL}$ pro mmol) gab man bei Raumtemperatur TBAF-Trihydrat (14.89 g, $47.2 \mathrm{mmol}$, 4.0 Äq.) zu und ließ für $20 \mathrm{~h}$ rühren. Anschließend wurde die Reaktionslösung auf Kieselgel (36.0 g, $3.0 \mathrm{~g}$ pro mmol) adsorbiert und das Rohprodukt säulenchromatographisch (EE/P) gereinigt. Man erhielt die Titelverbindung (1.84 g, $9.51 \mathrm{mmol}, 81 \%$ ) als ein leicht gelbliches Ö1.

\section{Variante 2:}

$\mathrm{Zu}$ einer Lösung von 1,5-Pentandiol (30.0 g, $30.3 \mathrm{~mL}, 288 \mathrm{mmol}, 1.0$ Äq.) in THF (400 mL, $1.4 \mathrm{~mL}$ pro mmol) wurde in kleinen Portionen $\mathrm{NaH}$ (11.8 g, $297 \mathrm{mmol}$, 1.03 Äq., $60 \%$-ig in Weißöl) gegeben und die Suspension für $10 \mathrm{~min}$ bei Raumtemperatur gerührt. Anschließend wurde eine Lösung Benzylbromid (19.32 g, $13.44 \mathrm{~mL}, 113 \mathrm{mmol}, 0.39$ Äq.) in THF (70.0 mL, $0.6 \mathrm{~mL}$ pro mmol Chlorid) zugetropft und die Suspension unter Reflux für $36 \mathrm{~h}$ gerührt. Es wurde auf $0{ }^{\circ} \mathrm{C}$ gekühlt und mit $\mathrm{H}_{2} \mathrm{O}(30.0 \mathrm{~mL})$ gequentscht. Die organische Phase wurde mit gesättigter $\mathrm{NaCl}$-Lösung gewaschen und die wässrige mit Ether $(3 \mathrm{x} 150 \mathrm{~mL}$ ) extrahiert. Die vereinigten organischen Phasen wurden über $\mathrm{MgSO}_{4}$ getrocknet und das Lösungsmittel im Vakuum entfernt. Das Rohprodukt wurde säulenchromatographisch (EE/P) gereinigt und man erhielt die Titelverbindung (19.2 g, $99.2 \mathrm{mmol}, 87.8 \%$ ) als ein farbloses Öl.

$\mathbf{R}_{\mathbf{f}}=0.15(\mathrm{EE} / \mathrm{P}=1: 4)$.

${ }^{1}$ H-NMR $\left(300 \mathrm{MHz}, \mathrm{CDCl}_{3}\right): \delta=1.22-1.72\left(\mathrm{~m}, 6 \mathrm{H}, 2-\mathrm{H}_{2}, 3-\mathrm{H}_{2}, 4-\mathrm{H}_{2}\right), 1.81\left(\mathrm{~s}_{\mathrm{b}}, 1 \mathrm{H}\right.$, $\mathrm{OH}), 3.48\left(\mathrm{t}, 2 \mathrm{H}, 5-\mathrm{H}_{2}, J=9.5 \mathrm{~Hz}\right), 3.63\left(\mathrm{t}, 2 \mathrm{H}, 1-\mathrm{H}_{2}, J=9.0 \mathrm{~Hz}\right), 4.50(\mathrm{~s}, 2 \mathrm{H}, \mathrm{Ph}-$ $\mathrm{CH}_{2}$ ), 7.26-7.36 (m, $\left.5 \mathrm{H}, \mathrm{Ph}-\mathrm{H}\right)$. 
${ }^{13}$ C-NMR (50.3 MHz, $\mathrm{CDCl}_{3}$ ): $\delta=21.99$ (C-3), 32.40 (C-4), 33.31 (C-2), 62.89 (C-1), $72.98(\mathrm{C}-5), 75.52\left(\mathrm{CH}_{2}-\mathrm{Ph}\right), 126.65,127.97,138.20(\mathrm{Ph}-\mathrm{C})$.

MS $\left(\mathrm{DCI}-\mathrm{NH}_{3}\right): m / z(\%)=212(19)[\mathrm{M}+18]^{+}, 195(2)[\mathrm{M}+1]^{+}$.

$\mathbf{C}_{12} \mathbf{H}_{18} \mathbf{O}_{2}$ (194.13)

\subsection{5-(Benzyloxy)pentanal (138)}

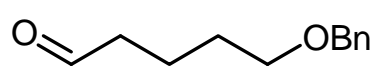

$\mathrm{Zu}$ einer Lösung des Alkohols 136 (18.5 g, $95.4 \mathrm{mmol}, 1.0$ Äq.) in $\mathrm{CH}_{2} \mathrm{Cl}_{2}$ (300 mL, $3.8 \mathrm{~mL}$ pro mmol) wurde bei $0{ }^{\circ} \mathrm{C}$ eine wässrige $1 \mathrm{M} \mathrm{KBr}$-Lösung $(9.54 \mathrm{~mL}$, $9.54 \mathrm{mmol}, 0.05$ Äq.), TEMPO (297 mg, $1.90 \mathrm{mmol}, 10 \mathrm{~mol}-\%)$ und eine frisch zubereitete Lösung bestehend aus einer 1:1-Mischung aus einer $0.5 \mathrm{M}$ wässrigen NaClO-Lösung (232.4 mL, 114.4 mmol, 1.2 Äq.) und gesättigter $\mathrm{NaHCO}_{3}(232.4 \mathrm{~mL}$ ) zugegeben. Das Reaktionsgemisch wurde $4 \mathrm{~h}$ sehr stark bei $0^{\circ} \mathrm{C}$ gerührt. Anschließend wurden die Phasen getrennt und die wässrige mit demselben Volumen an Ether extrahiert, die vereinigten organischen Phasen mit $\mathrm{H}_{2} \mathrm{O}$ und gesättigter $\mathrm{NaCl}$ Lösung gewaschen und über $\mathrm{MgSO}_{4}$ getrocknet. Das Lösungsmittel wurde im Vakuum entfernt und das Rohprodukt säulenchromatographisch (EE/P) gereinigt. Man erhielt die Titelverbindung (15.1 g, $78.6 \mathrm{mmol}, 82 \%)$ als ein leicht orangenes Öl.

$\mathbf{R}_{\mathbf{f}}=0.53(\mathrm{EE} / \mathrm{P}=1: 4)$.

${ }^{1}$ H-NMR (300 MHz, CDCl $)$ ): $\delta=1.46-1.62\left(\mathrm{~m}, 4 \mathrm{H}, 3-\mathrm{H}_{2}, 4-\mathrm{H}_{2}\right), 2.40\left(\mathrm{dt}, 2 \mathrm{H}, 2-\mathrm{H}_{2}\right.$, $J=2.0,6.8 \mathrm{~Hz}$ ), 3.37 (t, $\left.2 \mathrm{H}, 5-\mathrm{H}_{2}, J=4.9 \mathrm{~Hz}\right), 7.31-7.39(\mathrm{~m}, 5 \mathrm{H}, \mathrm{Ph}-\mathrm{H}), 9.72$ (d, 1 $\mathrm{H}, 1-\mathrm{H}, J=2.0 \mathrm{~Hz})$.

${ }^{13}$ C-NMR (50.3 MHz, $\mathrm{CDCl}_{3}$ ): $\delta=18.63$ (C-3), 29.52 (C-4), 44.35 (C-2), 72.04 (C-5), $74.9\left(\mathrm{CH}_{2} \mathrm{Ph}\right), 126.98,128.54,138.22(\mathrm{Ph}-\mathrm{C}), 202.12(\mathrm{C}=\mathrm{O})$.

MS $\left(\mathrm{DCI}-\mathrm{NH}_{3}\right): \mathrm{m} / \mathrm{z}(\%)=402(6)[2 \mathrm{M}+18]^{+}, 210(100)[\mathrm{M}+18]^{+}, 193(5)[\mathrm{M}+1]^{+}$.

$\mathbf{C}_{12} \mathbf{H}_{16} \mathbf{O}_{2}(192.12)$ 


\section{7. ((5,5-Dimethoxypentyloxy)methyl)benzol (139)}

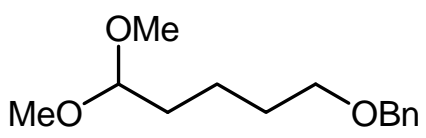

K-10 Montmorellonit-Pulver (71.9 g, $1.38 \mathrm{~g}$ pro mmol Aldehyd) wurde mit $\mathrm{CH}(\mathrm{OMe})_{3}$ (54.2 $\mathrm{mL}, 1.04 \mathrm{~mL}$ pro mmol Aldehyd) und $\mathrm{MeOH}(54.2 \mathrm{~mL}$. $1.04 \mathrm{~mL}$ pro $\mathrm{mmol}$ Aldehyd) gemischt. Die resultierende Mischung wurde einige Minuten gerührt und dann abfiltriert. Der nasse Filterkuchen wurde sofort weiterverarbeitet. Der Aldehyd 138 (10.0 g, 52.1 mmol, 1.0 Äq.) wurde mit 2/3 der Menge der Suspension aus K-10 Montmorellonit und $\mathrm{CH}(\mathrm{OMe})_{3}$ in $\mathrm{CH}_{2} \mathrm{Cl}_{2}(220 \mathrm{~mL}, 4.2 \mathrm{~mL}$ pro mmol) für $48 \mathrm{~h}$ bei Raumtemperatur gerührt. Die Suspension wurde über Celite 521 filtriert, hintereinander mit gesättigter $\mathrm{NaHCO}_{3^{-}}, \mathrm{H}_{2} \mathrm{O}$ und $\mathrm{NaCl}$-Lösung gewaschen, über $\mathrm{MgSO}_{4}$ getrocknet und das Lösungsmittel im Vakuum entfernt. Das Rohprodukt wurde säulenchromatographisch $(\mathrm{EE} / \mathrm{P})$ gereinigt und man erhielt die Titelverbindung (9.84 g, 41.3 mmol, 79 \%) als leicht gelbliches Öl.

$\mathbf{R}_{\mathbf{f}}=0.58(\mathrm{EE} / \mathrm{P}=1: 6)$.

UV $\left(\mathrm{CH}_{3} \mathrm{CN}\right): \lambda_{\max }(\lg \varepsilon): 205.5 \mathrm{~nm}(0.407), 251.5$ (0.013), 257.5 (0.013), 263.0 $(0.010)$.

IR (KBr): $v=2945 \mathrm{~cm}^{-1}, 1454,1363,1119,736,698$.

${ }^{1} \mathbf{H}-\mathrm{NMR}\left(300 \mathrm{MHz}, \mathrm{CDCl}_{3}\right): \delta=1.29-1.59\left(\mathrm{~m}, 6 \mathrm{H}, 2-\mathrm{H}_{2}, 3-\mathrm{H}_{2}, 4-\mathrm{H}_{2}\right), 3.24$ (s, $6 \mathrm{H}, 2$ x $\mathrm{OCH}_{3}$ ), 3.37 (t, $2 \mathrm{H}, 5-\mathrm{H}_{2}, J=5.5 \mathrm{~Hz}$ ), 4.19 (t, $\left.1 \mathrm{H}, 1-\mathrm{H}, J=6.5 \mathrm{~Hz}\right), 4.63(\mathrm{~s}, 2 \mathrm{H}$, $\left.\mathrm{CH}_{2}-\mathrm{Ph}\right), 7.31-7.39$ (m, $\left.5 \mathrm{H}, \mathrm{Ph}-\mathrm{H}\right)$.

${ }^{13}$ C-NMR (50.3 MHz, $\mathrm{CDCl}_{3}$ ): $\delta=16.43$ (C-3), 30.20 (C-4), 34.12 (C-2), 53.47 $\left(\mathrm{OCH}_{3}\right), 72.08$ (C-5), $74.94\left(\mathrm{CH}_{2}-\mathrm{Ph}\right), 127.23,129.04,136.99(\mathrm{Ph}-\mathrm{C})$.

MS $\left(\mathrm{DCI}-\mathrm{NH}_{3}\right): m / z(\%)=495(7)[2 \mathrm{M}+18]^{+}, 256(9)[\mathrm{M}+18]^{+}$.

$\mathrm{C}_{14} \mathbf{H}_{22} \mathbf{O}_{3}(238.16)$ 


\subsection{4,5-Dihydroxypentylacetat (163)}<smiles>CC(=O)OCCCC(O)CO</smiles>

$\mathrm{Zu}$ einer refluxierenden Lösung von Acetanhydrid $(170 \mathrm{~mL}$. $0.34 \mathrm{~mL}$ pro mmol Alkohol) und Zinkchlorid (0.83 g, $6.1 \mathrm{mmol}, \quad 0.12$ Äq.) wurde langsam Tetrahydrofurfurylalkohol (51.0 g, 48.4 mL, 500 mmol, 1.0 Äq.) zugetropft und die Reaktionslösung für weitere $24 \mathrm{~h}$ unter Rühren refluxiert. Anschließend wurde fraktioniert destilliert. Man erhielt Pentan-1,2,5-tiyltriacetat (56.5 g, 230 mmol, 70 \%) und 4,5-Dihydroxypentylacetat (12.6 g, $78 \mathrm{mmol}, 16 \%$ ) als farbloses Öl.

$\mathbf{R}_{\mathbf{f}}=0.44(\mathrm{EE} / \mathrm{P}=1: 2)$.

Sdp. $=67^{\circ} \mathrm{C}$ bei 3.5 Torr.

${ }^{1}$ H-NMR $\left(300 \mathrm{MHz}, \mathrm{CDCl}_{3}\right): \delta=1.52-1.66\left(\mathrm{~m}, 1 \mathrm{H}, 3-\mathrm{H}_{\mathrm{a}}\right)$, 1.82-2.09 (m, $3 \mathrm{H}, 3-\mathrm{H}_{\mathrm{b}}$, 4- $\left.\mathrm{H}_{2}\right), 2.07$ (s, $\left.3 \mathrm{H}, \mathrm{CH}_{3}\right), 3.72-4.19\left(\mathrm{~m}, 1-\mathrm{H}_{2}, 2-\mathrm{H}, 5-\mathrm{H}_{2}\right)$.

$\mathrm{C}_{11} \mathbf{H}_{18} \mathbf{O}_{6}(246.11)$ 


\section{Synthese des Dienophils 122}

\subsection{Tetrahydrofuran-2-ol (141)}

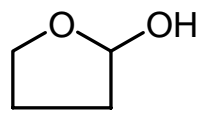

$\mathrm{Zu}$ einer rührenden Lösung von $\gamma$-Butyrolacton (10.0 g, $116 \mathrm{mmol}, 1.0$ Äq.) in $\mathrm{CH}_{2} \mathrm{Cl}_{2}$ (116 mL, $1.0 \mathrm{~mL}$ pro mmol) wurde bei $-78^{\circ} \mathrm{C}$ langsam DIBAH (136 mL, $136 \mathrm{mmol}$, 1.17 Äq., $1 \mathrm{M}$ Lösung in Hexan) zugetropft. Die Reaktionslösung wurde $1 \mathrm{~h}$ bei $-78^{\circ} \mathrm{C}$ gerührt, auf Raumtemperatur gebracht und anschließend mit $\mathrm{MeOH}(90.0 \mathrm{~mL})$ versetzt, wobei die Lösung zu gelieren begann. Darauf hin wurde soviel einer gesättigten $\mathrm{K}^{+} / \mathrm{Na}^{+}$-Tartrat-Lösung dazugegeben, bis man eine klare Lösung erhielt. Dazu wurde weitere $2 \mathrm{~h}$ bei Raumtemperatur gerührt. Die Phasen wurden getrennt und die wässrige mit $\mathrm{CH}_{2} \mathrm{Cl}_{2}(3 \times 300 \mathrm{~mL})$ extrahiert. Die vereinigten organischen Phasen wurden über $\mathrm{MgSO}_{4}$ getrocknet, das Lösungsmittel im Vakuum entfernt und das Rohprodukt säulenchromatographisch (EE/P) gereinigt. Man erhielt die Titelverbindung (7.26 g, $82.5 \mathrm{mmol}, 71 \%$ ) als farbloses Öl.

$\mathbf{R}_{\mathbf{f}}=0.35(\mathrm{EE} / \mathrm{P}=1: 4)$.

${ }^{1} \mathbf{H}-\mathbf{N M R}\left(300 \mathrm{MHz}, \mathrm{CDCl}_{3}\right): \delta=1.52-1.66\left(\mathrm{~m}, 1 \mathrm{H}, 3-\mathrm{H}_{\mathrm{a}}\right), 1.82-2.09\left(\mathrm{~m}, 3 \mathrm{H}, 3-\mathrm{H}_{\mathrm{b}}\right.$, 4- $\left.\mathrm{H}_{2}\right), 2.07$ (s, $\left.3 \mathrm{H}, \mathrm{CH}_{3}\right), 3.72-4.19\left(\mathrm{~m}, 1-\mathrm{H}_{2}, 2-\mathrm{H}, 5-\mathrm{H}_{2}\right)$.

MS $\left(\mathrm{DCI}-\mathrm{NH}_{3}\right): m / z(\%)=88(100)[\mathrm{M}-18+18]^{+}$.

$\mathbf{C}_{4} \mathbf{H}_{8} \mathbf{O}_{2}(88.05)$

\subsection{5-Methoxypent-4-en-1-ol (148)}

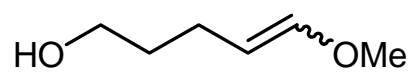

In einen getrockneten Kolben wurde (Methoxymethyl)triphenylphosphoniumchlorid (33.0 g, 96.5 mmol, 2.4 Äq.) für $1 \mathrm{~h}$ an die Hochvakuumpumpe angeschlossen, um alle 
Feuchtigkeit zu entfernen. Anschließend wurde THF $(200 \mathrm{~mL}, 2.0 \mathrm{~mL}$ pro mmol Chlorid) dazugegeben und die Suspension auf $-78^{\circ} \mathrm{C}$ gekühlt. n-BuLi (38.6 mL, 120.6 mmol, 3.0 Äq.) wurde tropfenweise zugegeben und die Reaktionsmischung für 30 min bei $-78^{\circ} \mathrm{C}$ gerührt. Danach wurde eine Lösung des Lactols 141 (3.54 g, $40.2 \mathrm{mmol}$, 1.0 Äq.) in THF $(50.0 \mathrm{~mL})$ zugetropft und $20 \mathrm{~h}$ bei Raumtemperatur gerührt. Es wurde mit gesättigter $\mathrm{NaHCO}_{3}$-Lösung $(200 \mathrm{~mL})$ gequentscht, die Lösung mit Ether $(200 \mathrm{~mL})$ verdünnt und die Phasen getrennt. Die wässrige Phase wurde mit Ether $(2 \times 200 \mathrm{~mL})$ extrahiert, die vereinigten organischen Phasen über $\mathrm{Na}_{2} \mathrm{SO}_{4}$ getrocknet, das Lösungsmittel im Vakuum entfernt und das Rohprodukt säulenchromatographisch (EE/P) gereinigt. Man erhielt die Titelverbindung (2.96 g, $25.5 \mathrm{mmol}, 63 \%$ ) als leicht orangenes, flüssiges Ö1.

$\mathbf{R}_{\mathbf{f}}=0.47(\mathrm{EE} / \mathrm{P}=1: 4)$.

${ }^{1} \mathbf{H}-\mathbf{N M R}\left(300 \mathrm{MHz}_{\mathrm{CDCl}}\right): \delta=1.60\left(\mathrm{~m}_{\mathrm{c}}, 2 \mathrm{H}, 2-\mathrm{H}_{2}\right), 2.02\left(\mathrm{q}, 1 \mathrm{H}, 3-\mathrm{H}_{\mathrm{a}}, J=7.5 \mathrm{~Hz}\right)$, 2.15 (q, $\left.2 \mathrm{H}, 3-\mathrm{H}_{\mathrm{b}}, \mathrm{OH}, J=7.5 \mathrm{~Hz}\right), 3.51$ (s, $\left.1.5 \mathrm{H}, \mathrm{OCH}_{3}\right), 3.59$ (s, $\left.1.5 \mathrm{H}, \mathrm{OCH}_{3}\right), 3.63$ (q, $\left.2 \mathrm{H}, 1-\mathrm{H}_{2}, J=6.5 \mathrm{~Hz}\right), 4.37(\mathrm{dt}, 0.5 \mathrm{H}, 4-\mathrm{H}, J=8.0,13.9 \mathrm{~Hz}), 4.73$ (dt, 0.5 H, 4-H, $J=7.3,12.8 \mathrm{~Hz}), 5.94(\mathrm{dt}, 0.5 \mathrm{H}, 5-\mathrm{H}, J=1.4,6.0 \mathrm{~Hz}), 6.32(\mathrm{dt}, 0.5 \mathrm{H}, 5-\mathrm{H}, J=1.2$, $13.6 \mathrm{~Hz})$.

${ }^{13}$ C-NMR (50.3 MHz, $\mathrm{CDCl}_{3}$ ): $\delta=19.60,23.92$ (C-3), 31.83, 33.41 (C-2), 55.82, 59.46 $\left(\mathrm{OCH}_{3}\right), 61.69,62.03$ (C-1), 102.19, 105.61 (C-4), 146.74, 147.25 (C-5).

MS $\left(\mathrm{DCI}-\mathrm{NH}_{3}\right): m / z(\%)=134(100)[\mathrm{M}+18]^{+}, 250(12)[2 \mathrm{M}+18]^{+}, 117(7)[\mathrm{M}+1]^{+}$, $151(5)[\mathrm{M}+17+18]^{+}$.

$\mathbf{C}_{6} \mathbf{H}_{12} \mathbf{O}_{2}(116.08)$

\subsection{1-Benzyloxy-5-methoxypent-4-en (122)}

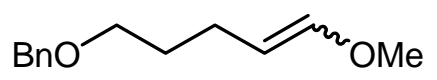

$\mathrm{Zu}$ einer Lösung von dem Alkohol 148 (2.83 g, 24.4 mmol, 1.0 Äq.) in THF (60.0 mL, $2.5 \mathrm{~mL}$ pro mmol) wurde $\mathrm{NaH}(1.17 \mathrm{~g}, 29.3 \mathrm{mmol}, 1.2$ Äq., $60 \%$-ig in Weißöl) und Benzylbromid (3.5 mL, 29.3 mmol, 1.2 Äq.) zugegeben und die Reaktionslösung für 
$20 \mathrm{~h}$ bei Raumtemperatur gerührt. Anschließend wurde mit der doppelten Menge Ether $(120 \mathrm{~mL})$ verdünnt und die organische Phase mit $\mathrm{H}_{2} \mathrm{O}(120 \mathrm{~mL})$ und gesättigter NaClLösung $(120 \mathrm{~mL})$ gewaschen. Es wurde über $\mathrm{Na}_{2} \mathrm{SO}_{4}$ getrocknet und das Lösungsmittel im Vakuum entfernt. Man erhielt die Titelverbindung (4.62 g, $22.4 \mathrm{mmol}, 92 \%$ ) als farbloses Öl.

$\mathbf{R}_{\mathbf{f}}=0.30(\mathrm{EE} / \mathrm{P}=1: 2)$.

${ }^{1}$ H-NMR (300 MHz, $\left.\mathrm{CDCl}_{3}\right): \delta=1.56\left(\mathrm{~m}_{\mathrm{c}}, 2 \mathrm{H}, 2-\mathrm{H}_{2}\right), 1.94\left(\mathrm{q}, 1 \mathrm{H}, 3-\mathrm{H}_{\mathrm{a}}, J=7.5 \mathrm{~Hz}\right)$, 2.04 (q, $1 \mathrm{H}, 3-\mathrm{H}_{\mathrm{b}}, J=7.5 \mathrm{~Hz}$ ), 3.37 (q, $\left.2 \mathrm{H}, 1-\mathrm{H}_{2}, J=5.6 \mathrm{~Hz}\right), 3.50\left(\mathrm{~s}, 1.5 \mathrm{H}, \mathrm{OCH}_{3}\right.$ ), 3.59 (s, $\left.1.5 \mathrm{H}, \mathrm{OCH}_{3}\right), 4.37$ (dt, $\left.0.5 \mathrm{H}, 4-\mathrm{H}, J=8.0,13.9 \mathrm{~Hz}\right), 4.67$ (dt, $0.5 \mathrm{H}, 4-\mathrm{H}, J=$ 7.3, $12.8 \mathrm{~Hz}$ ), 4.69 (s, $2 \mathrm{H}, \mathrm{CH}_{2}-\mathrm{Ph}$ ), 5.94 (dt, $\left.0.5 \mathrm{H}, 5-\mathrm{H}, J=1.4,6.0 \mathrm{~Hz}\right), 6.41$ (dt, 0.5 $\mathrm{H}, 5-\mathrm{H}, J=1.2,13.6 \mathrm{~Hz}), 7.33-7.42(5 \mathrm{H}, \mathrm{Ph}-\mathrm{H})$.

${ }^{13} \mathrm{C}-\mathrm{NMR}\left(50.3 \mathrm{MHz}, \mathrm{CDCl}_{3}\right): \delta=24.92,26.31$ (C-3), 29.83, 31.53 (C-2), 55.82, $58.86\left(\mathrm{OCH}_{3}\right), 72.69,74.03(\mathrm{C}-1), 75.87\left(\mathrm{CH}_{2} \mathrm{Ph}\right), 102.19,105.44(\mathrm{C}-4), 125.79$, 126.90, 183.21 (Ph-C), 145.64, 149.33 (C-5).

MS (70 eV, EI): m/z (\%) = 91 (72) $\left[\mathrm{M}-\mathrm{OCH}_{2} \mathrm{CH}_{2} \mathrm{CH}_{2} \mathrm{CH}=\mathrm{CHOMe}\right]^{+}, 107$ (18) [M$\left.\mathrm{CH}_{2} \mathrm{CH}_{2} \mathrm{CH}_{2} \mathrm{CH}=\mathrm{CHOMe}\right]^{+}$.

$\mathbf{C}_{13} \mathbf{H}_{18} \mathbf{O}_{2}$ (206.13) 


\section{B) Versuche zur Synthese von Camptothecin (44)}

\section{Synthese des Modelsystems 218 zur Überprüfung der Totalsynthese von Camptotecin}

\subsection{2-(3,4-Dihydroisochinolin-1(2H)-yliden)essigsäureethylester (214)}<smiles>CCOC(=O)/C=C1\NCCc2ccccc21</smiles>

$\mathrm{Zu}$ einer auf $0^{\circ} \mathrm{C}$ gekühlten Lösung von 3-Oxo-3-(phenylethylamino)propansäureethylester (4.5 g, 19.0 mmol, 1.0 Äq.) und DMAP (6.96 g, $57.0 \mathrm{mmol}, 3.0$ Äq.) in $\mathrm{CH}_{2} \mathrm{Cl}_{2}\left(513 \mathrm{~mL}, 27.0 \mathrm{~mL}\right.$ pro mmol Ester) wurde innerhalb von 15 min eine auf $0{ }^{\circ} \mathrm{C}$ vorgekühlte Lösung von $\mathrm{Tf}_{2} \mathrm{O}$ (16.1 mL, $95.0 \mathrm{mmol}, 5.0$ Äq.) in $\mathrm{CH}_{2} \mathrm{Cl}_{2}$ (17.3 mL, $0.91 \mathrm{~mL}$ pro mmol Ester) zugetropft. Man ließ auf Raumtemperatur kommen und rührte die Reaktionsmischung für 5 Tage. Anschließend wurde mit $\mathrm{CH}_{2} \mathrm{Cl}_{2}(750 \mathrm{~mL})$ verdünnt, mit gesättigter $\mathrm{Na}_{2} \mathrm{CO}_{3}$-Lösung (400 mL), 20 \%-iger Essigsäure $(400 \mathrm{~mL})$ und wieder mit gesättigter $\mathrm{Na}_{2} \mathrm{CO}_{3}$-Lösung (400 mL) gewaschen und über $\mathrm{Na}_{2} \mathrm{SO}_{4}$ getrocknet. Man entfernte das Lösungsmittel im Vakuum und reinigte das Rohprodukt säulenchromatographisch (Alox neutral, EE). Man erhielt die Titelverbindung (2.88 g, $13.3 \mathrm{mmol}, 70 \%$ ) als braun-rötliches Öl.

$\mathbf{R}_{\mathbf{f}}=0.94$ (Alox N, EtOAc).

IR (Film): $v=3295 \mathrm{~cm}^{-1}(\mathrm{NH}), 2978(\mathrm{CH}), 1649,1601(\mathrm{C}=\mathrm{C}), 1568,1483,1266$, 1160 (C-OEt), 1034 (O-Et), 771 (ortho disubst. Ar).

UV ( $\left.\mathrm{CH}_{3} \mathrm{CN}\right): \lambda_{\max }(\lg \varepsilon): 205.5 \mathrm{~nm}$ (4.347), 247.0 (4.035), 332.5 (3.998).

${ }^{1}$ H-NMR $\left(200 \mathrm{MHz} \mathrm{CDCl}_{3}\right): \delta=1.30\left(\mathrm{t}, J=7.2 \mathrm{~Hz}, 3 \mathrm{H}, \mathrm{CH}_{3}\right), 2.91(\mathrm{t}, J=6.4 \mathrm{~Hz}, 2$ $\left.\mathrm{H}, 4-\mathrm{H}_{2}\right), 3.44\left(\mathrm{td}, J=6.4,3.3 \mathrm{~Hz}, 2 \mathrm{H}, 3-\mathrm{H}_{2}\right), 4.17$ (q, $\left.J=7.2 \mathrm{~Hz}, 2 \mathrm{H}, \mathrm{OCH}_{2} \mathrm{CH}_{3}\right)$, 5.17 (s, $1 \mathrm{H}, 2$ '-H), $7.14-7.41$ (m, $3 \mathrm{H}, \mathrm{Ar}-\mathrm{H}), 7.67$ (dd, $J=7.6,1.3 \mathrm{~Hz}, 1 \mathrm{H}, 8-\mathrm{H})$, $9.06\left(\mathrm{~s}_{\mathrm{b}}, 1 \mathrm{H}, \mathrm{NH}\right)$. 
${ }^{13}$ C-NMR (50.3 MHz, $\left.\mathrm{CDCl}_{3}\right): \delta=14.65\left(\mathrm{CH}_{3}\right), 29.03(\mathrm{C}-4), 38.74(\mathrm{C}-3), 58.63$ $\left(\mathrm{OCH}_{2}\right), 78.11$ (C-2'), 125.2 (C-8), 126.9 (C-6), 128.2 (C-7), 129.6 (C-8a), 130.3 (C5), $136.4(\mathrm{C}-4 \mathrm{a}), 156.2(\mathrm{C}-1), 171.3\left(\mathrm{CO}_{2} \mathrm{Et}\right)$.

MS (70 eV, EI): $m / z(\%)=217.1(36)[\mathrm{M}]^{+}, 172.1(34)[\mathrm{M}-\mathrm{OEt}]^{+}, 145.1(100)[\mathrm{M}-$ $\mathrm{CO} 2 \mathrm{Et}+1]^{+}, 115.1(44)\left[\mathrm{M}-\mathrm{CO}_{2} \mathrm{Et}-\mathrm{CH}_{2} \mathrm{NH}\right]^{+}$.

$\mathrm{C}_{13} \mathrm{H}_{15} \mathrm{NO}_{2}$ (217.11)

\subsection{2-(1,2,3,4-Tetrahydroisochinolin-1-yl)essigsäureethylester (215)}

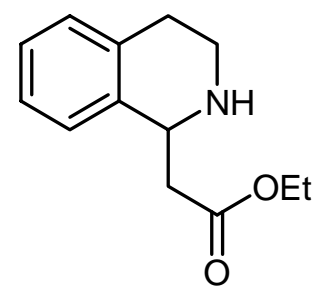

Variante 1:

Analog AAV 3 setzte man den Ester 214 (1.5 g, 6.8 mmol, 1.0 Äq.) mit $\mathrm{LiAlH}_{4}$ (0.78 g, 20.5 mmol, 3.0 Äq.) in THF (70.0 mL) innerhalb von $5 \mathrm{~h}$ um. Man erhielt die Titelverbindung (1.2 g, $6.8 \mathrm{mmol}, 97 \%)$ als ein hellbraunes Öl.

Variante 2:

Zu einer Lösung des Esters 214 (2.2 g, 10.0 mmol, 1.0 Äq.) in Ethanol (15.0 mL) und Eisessig $(11.0 \mathrm{~mL})$ in einem Hydrierkolben gab man Pd/C (0.44 g, 4.66 mol-\%, 10 \%ig) und ließ die Suspension unter einer $\mathrm{H}_{2}$-Atmosphäre (4.0 bar) schütteln. Der Katalysator wurde abfiltriert und Ethanol im Vakuum entfernt. Die verbleibende Lösung wurde mit $\mathrm{H}_{2} \mathrm{O}(25 \mathrm{~mL})$ verdünnt und mit $\mathrm{K}_{2} \mathrm{CO}_{3}$ vorsichtig auf $\mathrm{pH}$ 8-10 gebracht. Nach Extraktion der wässrigen Phase mit EE $(2 \times 25 \mathrm{~mL})$ wurden die vereinigten organischen Phasen über $\mathrm{Na}_{2} \mathrm{SO}_{4}$ getrocknet und das Lösungsmittel im Vakuum entfernt. Das Rohprodukt wurde säulenchromatographisch (EE) gereinigt. Man erhielt die Titelverbindung (1.6 g, 7.3 mmol, 73 \%) als hellbraunes Öl. 
$\mathbf{R}_{\mathbf{f}}=0.28($ EtOAc $/ \mathrm{MeOH} 4: 1)$.

IR (Film): $v=3348 \mathrm{~cm}^{-1}(\mathrm{NH}), 2980(\mathrm{CH}), 1731(\mathrm{C}=\mathrm{O}), 1655,1455,1370,1176(\mathrm{C}-$ OEt), 1036 (O-Et), 747 (ortho disubst. Ar).

UV $\left(\mathrm{CH}_{3} \mathrm{CN}\right): \lambda_{\max }(\lg \varepsilon): 265.5 \mathrm{~nm}$ (3.168), 272.5 (3.506), 296.0 (3.559).

${ }^{1}$ H-NMR $\left(200 \mathrm{MHz}, \mathrm{CDCl}_{3}\right): \delta=1.27\left(\mathrm{t}, J=7.3 \mathrm{~Hz}, 3 \mathrm{H}, \mathrm{CH}_{3}\right), 2.27(\mathrm{~s}, 1 \mathrm{H}, \mathrm{NH})$, $2.65-3.30$ (m, $6 \mathrm{H}, 3-\mathrm{H}_{2}, 4-\mathrm{H}_{2}, 2^{\prime}-\mathrm{H}_{2}$ ), 4.18 (q, J = 7.3 Hz, $2 \mathrm{H}, \mathrm{OCH}_{2} \mathrm{CH}_{3}$ ), 4.47 (dd, $J=9.4,3.6 \mathrm{~Hz}, 1 \mathrm{H}, 1-\mathrm{H}), 7.05-7.19$ (m, $4 \mathrm{H}, \mathrm{Ar}-\mathrm{H})$.

${ }^{13}$ C-NMR (50.3 MHz, $\left.\mathrm{CDCl}_{3}\right): \delta=14.18\left(\mathrm{CH}_{3}\right), 29.76$ (C-4), 40.59, 41.34 (C-2', C-3), $52.64(\mathrm{C}-1), 60.53\left(\mathrm{OCH}_{2}\right), 125.9,126.3,129.4(\mathrm{Ar}), 135.4(\mathrm{C}-8 \mathrm{a}), 137.5(\mathrm{C}-4 \mathrm{a})$, $172.3\left(\mathrm{CO}_{2} \mathrm{Et}\right)$.

MS (70 eV, EI): $m / z(\%)=219.2(4)[\mathrm{M}]^{+}, 132.1(100)\left[\mathrm{M}-\mathrm{CH}_{2} \mathrm{CO}_{2} \mathrm{Et}\right]^{+}, 117.1(8)$

$\left[\mathrm{M}-\mathrm{CH}_{2} \mathrm{CO}_{2} \mathrm{Et}-\mathrm{NH}\right]^{+}$.

$\mathrm{C}_{13} \mathrm{H}_{17} \mathrm{NO}_{2}$ (219.13)

\subsection{2-(1,2,3,4-Tetrahydroisochinolin-1-yl)ethanol (216)}<smiles>OCCC1NCCc2ccccc21</smiles>

Analog AAV 3 setzte man den Ester 215 (1.5 g, 6.8 mmol, 1.0 Äq.) mit $\mathrm{LiAlH}_{4}$ (0.78 g, $20.5 \mathrm{mmol}, 3.0$ Äq.) in THF (70.0 mL) innerhalb von $5 \mathrm{~h}$ um. Man erhielt die Titelverbindung (1.2 g, $6.8 \mathrm{mmol}, 97 \%$ ) als ein hellbraunes Öl.

$\mathbf{R}_{\mathbf{f}}=0.22(\mathrm{EtOAc} / \mathrm{MeOH} 4: 1)$.

IR (Film): $v=3297 \mathrm{~cm}^{-1}(\mathrm{NH}), 2924(\mathrm{CH}), 1603,1454,1066\left(\mathrm{CH}_{2}-\mathrm{OH}\right), 742$ (ortho disubst. Ar).

UV $\left(\mathrm{CH}_{3} \mathrm{CN}\right): \lambda_{\max }(\lg \varepsilon): 194.0 \mathrm{~nm}$ (4.515), 257.5 (2.963), 265.0 (2.974), 272.5 (2.967), 294.5 (2.927). 
${ }^{1} \mathbf{H}-\mathbf{N M R}\left(200 \mathrm{MHz}, \mathrm{CDCl}_{3}\right): \delta=1.83-2.02(\mathrm{~m}, 1 \mathrm{H}, 1$ '-H), $2.02-2.30(\mathrm{~m}, 1 \mathrm{H}, 1$ 'H), $2.62-2.95\left(\mathrm{~m}, 2 \mathrm{H}, 4-\mathrm{H}_{2}\right), 2.95-3.13(\mathrm{~m}, 1 \mathrm{H}, 3-\mathrm{H}), 3.15-3.23(\mathrm{~m}, 1 \mathrm{H}, 3-\mathrm{H})$, $3.65\left(\mathrm{~s}_{\mathrm{b}}, 1 \mathrm{H}, \mathrm{OH}\right), 3.72-4.00\left(\mathrm{~m}, 2 \mathrm{H}, 2^{\prime}-\mathrm{H}_{2}\right), 4.27(\mathrm{dd}, \mathrm{J}=9.3,3.1 \mathrm{~Hz}, 1 \mathrm{H}, 1-\mathrm{H})$, $6.97-7.35$ (m, 4 H, Ar-H).

${ }^{13}$ C-NMR (50.3 MHz, $\mathrm{CDCl}_{3}$ ): $\delta=29.25$ (C-4), 35.97 (C-1'), 39.49 (C-3), 56.86 (C1), 62.77 (C-2'), 126.0, 126.2, 126.2, 129.4 (Ar), 134.8, 138.0 (C-4a, C-8a).

MS (70 eV, EI): $m / z(\%)=355.4(2)[2 \mathrm{M}+1]^{+}, 178.1(100)[\mathrm{M}+1]^{+}$.

$\mathrm{C}_{11} \mathbf{H}_{15} \mathrm{NO}$ (177.12)

\subsection{Benzyl-1-(2-hydroxyethyl)-3,4-dihydroisochinolin-2(1H)-carboxylat (217)}<smiles>O=[N+]1CCc2ccccc2C1CCO</smiles>

$\mathrm{Zu}$ einem heftig gerührten Zweiphasensystem von 216 ( $1.0 \mathrm{~g}, 5.65 \mathrm{mmol}, 1.0$ Äq.) in $\mathrm{CH}_{2} \mathrm{Cl}_{2}(22.0 \mathrm{~mL})$ und gesättigter $\mathrm{NaHCO}_{3}$-Lösung $(22.0 \mathrm{~mL})$ wurde bei Raumtemperatur Chlorameisensäurebenzylester (1.1 g, $0.88 \mathrm{~mL}, 6.22 \mathrm{mmol}, \quad 1.2$ Äq.) tropfenweise gegeben. Die Reaktionslösung wurde für $3.5 \mathrm{~h}$ unter Rückfluss gerührt, anschließend die Phasen getrennt und die wässrige mit $\mathrm{CH}_{2} \mathrm{Cl}_{2}(3 \times 20 \mathrm{~mL})$ extrahiert. Die vereinigten organischen Phasen wurden über $\mathrm{Na}_{2} \mathrm{SO}_{4}$ getrocknet und das Lösungsmittel im Vakuum entfernt. Das Rohprodukt wurde säulenchromatographisch (EE/P) gereinigt. Man erhielt die Titelverbindung (1.26 g, $4.28 \mathrm{mmol}, 75 \%$ ) als orangenes Öl.

$\mathbf{R}_{\mathbf{f}}=0.63($ EtOAc).

IR (Film): $v=3444 \mathrm{~cm}^{-1}(\mathrm{OH}), 2947(\mathrm{CH}), 1695(\mathrm{C}=\mathrm{O}), 1428,1333$ (O-CO), 1228 ( $\left.\mathrm{CH}_{2}-\mathrm{OCO}\right), 1122$ (O-CO st sy), $1055\left(\mathrm{CH}_{2}-\mathrm{OH}\right), 761$ (ortho disubst. Ar), 698 (monosubst. Ar).

UV $\left(\mathrm{CH}_{3} \mathrm{CN}\right): \lambda_{\max }(\lg \varepsilon): 257.5 \mathrm{~nm}(2.729), 263.5$ (2.763), 271.0 (2.626). 
${ }^{1} \mathbf{H}-\mathbf{N M R}\left(200 \mathrm{MHz}, \mathrm{CDCl}_{3}\right): \delta=1.71-1.90(\mathrm{~m}, 1 \mathrm{H}, 1$ '-H), $1.92-2.25$ (m, 1 H. 1'H), 2.75 (dt, $J=16.1,4.2 \mathrm{~Hz}, 1 \mathrm{H}, 4-\mathrm{H}), 2.85-3.05$ (m, $1 \mathrm{H}, 4-\mathrm{H}), 3.16-3.34$ (m 1 H, 3-H), $3.47-3.77$ (m, 2 H, 2'- $\left.\mathrm{H}_{2}\right), 3.80-4.04$ ( $\left.\mathrm{s}_{\mathrm{b}}, 1 \mathrm{H}, \mathrm{OH}\right), 4.13$ (dt, J = 13.2, 4.2 $\mathrm{Hz}, 1 \mathrm{H}, 3-\mathrm{H}), 5.17$ (d, $\left.J=12.3 \mathrm{~Hz}, 1 \mathrm{H}, \mathrm{PhCH}_{2}\right), 5.25$ (d, $J=12.3 \mathrm{~Hz}, 1 \mathrm{H}, \mathrm{PhCH}_{2}$ ), $5.36(\mathrm{dd}, J=11.9,3.3 \mathrm{~Hz}, 1 \mathrm{H}, 1-\mathrm{H}), 7.07-7.25$ (m, 4 H, Ar-H), $7.31-7.45$ (m, 5 H, $\mathrm{Ph}-\mathrm{H})$.

${ }^{13}$ C-NMR (50.3 MHz, $\left.\mathrm{CDCl}_{3}\right): \delta=28.90$ (C-4), 38.59, 39.22 (C-1', C-3), 51.52 (C-1),

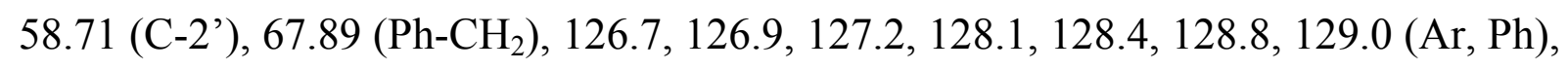
133.9, 136.7, 137.5 (C-4a, C-8a, CPh-CH$), 157.0\left(\mathrm{NCO}_{2}\right)$

MS (70 eV, EI): $m / z(\%)=640.5$ (1) $[2 \mathrm{M}+18]^{+}, 329.3(51)[\mathrm{M}+18]^{+}, 312.2(31)$ $[\mathrm{M}+1]^{+}, 178.1(100)[\mathrm{M}-\mathrm{Cbz}+2 \mathrm{H}]^{+}$.

$\mathrm{C}_{19} \mathrm{H}_{21} \mathrm{NO}_{3}(311.15)$

\subsection{Benzyl-1-(2-oxethyl)-3,4-dihydroisochinolin-2(1H)-carboxylat (218)}<smiles>O=CCC1c2ccccc2CCN1C(=O)[O-]</smiles>

$\mathrm{Zu}$ einer Suspension des Dess-Martin-Reagenzes (636 mg, $1.5 \mathrm{mmol}, 1.5$ Äq.) in $\mathrm{CH}_{2} \mathrm{Cl}_{2}(10.0 \mathrm{~mL})$ wurde bei $0{ }^{\circ} \mathrm{C}$ tropfenweise eine Lösung von dem Alkohol 217 (296 mg, $1.0 \mathrm{mmol}, 1.0 \mathrm{Äq}$ ) in $\mathrm{CH}_{2} \mathrm{Cl}_{2} \quad(5.0 \mathrm{~mL})$ gegeben. Man ließ auf Raumtemperatur kommen und $1.5 \mathrm{~h}$ rühren. Das Reaktionsgemisch wurde mit Ether (10.0 mL) verdünnt und mit $1 \mathrm{~N} \mathrm{NaOH-Lösung}(20.0 \mathrm{~mL})$ extrahiert. Die organische Phase wurde mit geättigter $\mathrm{NaCl}$-Lösung $(20.0 \mathrm{~mL})$ gewaschen und über $\mathrm{Na}_{2} \mathrm{SO}_{4}$ getrocknet. Das Lösungsmittel wurde im Vakuum entfernt und der Rückstand säulenchromatographisch (EE/P) gereinigt. Man erhielt die Titelverbindung (241 mg, $0.78 \mathrm{mmol}, 78 \%$ ) als farbloses Öl. 
$\mathbf{R}_{\mathbf{f}}=0.65$ (EtOAc).

IR (Film): $v=2932 \mathrm{~cm}^{-1}(\mathrm{CH}), 1697(\mathrm{C}=\mathrm{O}, \mathrm{Cbz}$ und $\mathrm{CHO}), 1428,1327(\mathrm{O}-\mathrm{CO})$, 1296, $1226\left(\mathrm{CH}_{2}-\mathrm{OCO}\right), 1122$ (O-CO), 765 (ortho disubst. Ar), 699 (monosubst. Ar).

UV $\left(\mathrm{CH}_{3} \mathrm{CN}\right): \lambda_{\max }(\lg \varepsilon): 257.0 \mathrm{~nm}$ (2.916), 263.5 (2.916), 271.5 (2.812).

${ }^{1} \mathbf{H}-\mathrm{NMR}\left(200 \mathrm{MHz}, \mathrm{CDCl}_{3}\right): \delta=3.97-4.33\left(\mathrm{~m}, 4 \mathrm{H}, 4-\mathrm{H}_{2}, 2{ }^{\prime}-\mathrm{H}_{2}\right), 3.20-3.55(\mathrm{~m}, 1$ H, 3-H), $2.70-3.09$ (m, $1 \mathrm{H}, 3-\mathrm{H}), 5.13$ (d, $J=12.3 \mathrm{~Hz}, 1 \mathrm{H}, \mathrm{PhCH}_{2^{-}}$), 5.20 (d, 12.3 $\left.\mathrm{Hz}, 1 \mathrm{H}, \mathrm{PhCH}_{2}-\right), 5.64-5.90$ (m, $\left.1 \mathrm{H}, 1-\mathrm{H}\right), 7.10$ - 7.25 (m, 4 H, Ar-H), 7.33 - 7.41 (m, 5 H, Ph-H), $9.69-9.94$ (m, 1 H, CHO).

${ }^{13}$ C-NMR (50.3 MHz, $\mathrm{CDCl}_{3}$ ): $\delta=28.47$ (C-4), 38.75 (C-3), 50.37 (C-2'), 51.13 (C1), $67.43\left(\mathrm{Ph}_{-} \mathrm{CH}_{2}\right), 126.7,127.2,127.9,128.1,128.2,128.5,129.0(\mathrm{Ar}, \mathrm{Ph}), 129.2(\mathrm{C}-$ 8a), $134.1\left(\mathrm{CPh}-\mathrm{CH}_{2}\right), 135.6$ (C-4a), $155.4\left(\mathrm{NCO}_{2}\right), 200.1$ (CHO).

MS $\left(\mathrm{DCI}-\mathrm{NH}_{3}\right) \mathrm{m} / \mathrm{z}(\%)=636.5(3)[2 \mathrm{M}+18]^{+}, 327.3(28)[\mathrm{M}+18]^{+}, 310.2(100)$ $[\mathrm{M}+1]^{+}, 266.2(20)\left[\mathrm{M}-\mathrm{CH}_{2} \mathrm{CHO}\right]^{+}$.

$\mathrm{C}_{19} \mathrm{H}_{19} \mathrm{NO}_{3}(309.14)$ 


\section{Versuche zur Synthese des Dienophils 129}

\subsection{2-Oxobuttersäuremethylester (219)}<smiles>CCC(=O)C(=O)OC</smiles>

$\mathrm{Zu}$ einer rührenden Lösung von $\alpha$-Ketobuttersäure (3.06 g, $30.0 \mathrm{mmol}, 1.0$ Äq.), 2,2Dimethoxypropan $(48.0 \mathrm{~mL}, 1.6 \mathrm{~mL}$ pro mmol) und $\mathrm{MeOH}(12.0 \mathrm{~mL}, 0.4 \mathrm{~mL}$ pro mmol) wurde TMSCl $(0.38 \mathrm{~mL}, 3.0 \mathrm{mmol}, 0.1$ Äq.) gegeben und die Reaktionsmischung für $24 \mathrm{~h}$ bei Raumtemperatur gerührt. Das Lösungsmittel wurde im Vakuum entfernt. Man erhielt die Titelverbindung (3.29 g, $28.4 \mathrm{mmol}, 95 \%$ ) als gelbliche Flüssigkeit.

$\mathbf{R}_{\mathbf{f}}=0.78(\mathrm{EE} / \mathrm{P}=1: 1)$.

${ }^{1}$ H-NMR $\left(300 \mathrm{MHz}, \mathrm{CDCl}_{3}\right): \delta=1.06\left(\mathrm{t}, 3 \mathrm{H}, \mathrm{CH}_{3}, J=10.4 \mathrm{~Hz}\right), 2.81$ (q, $2 \mathrm{H}, \mathrm{CH}_{2}, J$ $=10.4 \mathrm{~Hz}), 3.80\left(\mathrm{~s}, 3 \mathrm{H}, \mathrm{OCH}_{3}\right)$.

${ }^{13}$ C-NMR (50.3 MHz, $\left.\mathrm{CDCl}_{3}\right): \delta=8.42(\mathrm{C}-4), 24.61(\mathrm{C}-3), 52.20\left(\mathrm{OCH}_{3}\right), 161.26(\mathrm{C}-$ 1), 197.18 (C-2).

$\mathbf{C}_{5} \mathbf{H}_{8} \mathbf{O}_{3}(116.05)$

\subsection{2-Acetoxybuttersäure (223)}<smiles>CCC(OC(C)=O)C(=O)O</smiles>

$\mathrm{Zu}$ einer Lösung von 2-Aminobuttersäure (3.09 g, $30.0 \mathrm{mmol}, 1.0$ Äq.) in Eisessig $(90.0 \mathrm{~mL}, 3.0 \mathrm{~mL}$ pro mmol) wurde portionsweise unter Kühlung mit einem EisWasser-Bad $\mathrm{NaNO}_{2}$ (4.14 g, 60.0 mmol, 2.0 Äq.) zugegeben. Es wurde für $1 \mathrm{~h}$ bei 
Raumtemperatur gerührt und anschließend die Essigsäure im Vakuum entfernt. Nach Zugabe von $\mathrm{H}_{2} \mathrm{O}(90.0 \mathrm{~mL})$ wurde die wässrige Phase mit EE (3 x $\left.90.0 \mathrm{~mL}\right)$ und Ether (3 x $90.0 \mathrm{~mL}$ ) extrahiert und die vereinigten organischen Phasen über $\mathrm{MgSO}_{4}$ getrocknet. Das Lösungsmittel wurde im Vakuum entfernt. Man erhielt die Titelverbindung (1.62 g, 11.2 mmol, 37 \%) als gelbliche Flüssigkeit.

$\mathbf{R}_{\mathbf{f}}=0.14(\mathrm{EE} / \mathrm{P}=1: 4)$.

${ }^{1}$ H-NMR $\left(300 \mathrm{MHz}, \mathrm{CDCl}_{3}\right): \delta=1.00(\mathrm{t}, 3 \mathrm{H}, 4-\mathrm{h}, J=11.8 \mathrm{~Hz}), 1.89\left(\mathrm{~m}_{\mathrm{c}}, 2 \mathrm{H}, 3-\mathrm{H}_{2}\right)$, $2.12\left(\mathrm{~s}, 3 \mathrm{H}, \mathrm{CH}_{3}\right), 4.95$ (q, $\left.1 \mathrm{H}, 2-\mathrm{H}, J=10.6 \mathrm{~Hz}\right), 9.48$ (s $\left., 1 \mathrm{H}, \mathrm{OH}\right)$.

${ }^{13}$ C-NMR (50.3 MHz, $\left.\mathrm{CDCl}_{3}\right): \delta=7.73(\mathrm{C}-4), 21.54\left(\mathrm{CH}_{3}\right), 22.88(\mathrm{C}-3), 86.72(\mathrm{C}-2)$, $173(\mathrm{C}=\mathrm{O}), 174.97(\mathrm{C}-1)$.

MS $(70 \mathrm{eV}, \mathrm{EI}): \mathrm{m} / \mathrm{z}(\%)=99(80)[\mathrm{M}-\mathrm{OAc}]^{+}, 131(48)\left[\mathrm{M}-\mathrm{CH}_{3}\right]^{+}$.

$\mathbf{C}_{6} \mathbf{H}_{10} \mathbf{O}_{4}(146.06)$ 


\section{Versuche zur Synthese des Aldehyds $\mathbf{1 3 0}$}

\subsection{4-Oxo-4-(phenylamino)but-2-ensäureethylester (200)}<smiles>CCOC(=O)/C=C/C(=O)Nc1ccccc1</smiles>

Fumarsäuremonoethylester (1.44 g, 10.0 mmol, 1.0 Äq.) wurde mit $\mathrm{SOCl}_{2}(3.26 \mathrm{~mL}$, $45.0 \mathrm{mmol}, 4.5$ Äq.) versetzt und für $20 \mathrm{~h}$ bei Raumtemperatur gerührt. Anschließend wurde für 3 h unter Rückfluß erhitzt. Nach Abkühlung wurde der Überschuß an $\mathrm{SOCl}_{2}$ im Vakuum entfernt. Der Rückstand wurde in $\mathrm{CH}_{2} \mathrm{Cl}_{2}(10 \mathrm{~mL})$ gelöst und bei $0{ }^{\circ} \mathrm{C} \mathrm{zu}$ einer Lösung von frisch destilliertem Anilin (1.00 mL, $11.0 \mathrm{mmol}, 1.1$ Äq.) und $\mathrm{NEt}_{3}$ (2.79 mL, $20.0 \mathrm{mmol}, 2.0$ Äq.) in $\mathrm{CH}_{2} \mathrm{Cl}_{2}(50 \mathrm{~mL}, 5.0 \mathrm{~mL}$ pro mmol Ester) tropfenweise über eine Transferkanüle gegeben. Die Reaktionsmischung wurde für $24 \mathrm{~h}$ bei Raumtemperatur gerührt, mit $5 \%$-iger $\mathrm{HCl}(25 \mathrm{~mL})$ gewaschen und die wässrige Phase mit $\mathrm{CH}_{2} \mathrm{Cl}_{2}(2 \times 25 \mathrm{~mL})$ extrahiert. Die vereinigten organischen Phasen wurden mit gesättigter $\mathrm{NaHCO}_{3}$-Lösung $(50 \mathrm{~mL})$ gewaschen und die wässrige Phase nochmals mit $\mathrm{CH}_{2} \mathrm{Cl}_{2}(2 \times 50 \mathrm{~mL})$ extrahiert. Die vereinigten organischen Phasen wurden über $\mathrm{Mg} \mathrm{SO}_{4}$ getrocknet und das Lösungsmittel im Vakuum entfernt. Der schwarze, klebrige Rückstand wurde säulenchromatographisch (EE/P) gereinigt und man erhielt die Titelverbindung (0.42 g, $1.90 \mathrm{mmol}, 19 \%)$ als orangenes Pulver.

$\mathbf{R}_{\mathbf{f}}=0.36(\mathrm{EE} / \mathrm{P}=1: 4)$.

IR (Film): $v=3489 \mathrm{~cm}^{-1}, 3256,1720,1699,1687,1672,1651,1638,1613,1556$, $1509,1240,1163,843,830$.

${ }^{1}$ H-NMR $\left(300 \mathrm{MHz}, \mathrm{CDCl}_{3}\right): \delta=1.33\left(\mathrm{t}, 3 \mathrm{H}, \mathrm{CH}_{3}, J=10.2 \mathrm{~Hz}\right), 4.28\left(\mathrm{Q}, 2 \mathrm{H}, \mathrm{CH}_{2}, J\right.$ $=10.2 \mathrm{~Hz}$ ), 7.0 (q, $2 \mathrm{H}, 2-\mathrm{H}, 3-\mathrm{H}, J=17.5 \mathrm{~Hz}), 7.30-7.71$ (m, $5 \mathrm{H}, \mathrm{Ph}-\mathrm{H})$.

${ }^{13}$ C-NMR (50.3 MHz, $\left.\mathrm{CDCl}_{3}\right): \delta=14.01\left(\mathrm{CH}_{3}\right), 61.38\left(\mathrm{CH}_{2}\right), 120.19$ (Ar-C), 124.90 (C-3), 128.93 (Ar-C), 130.82 (C-2), 137.24 (Ar-C), 137.51 (Ar-C), 161.85 (C-4), $165.96(\mathrm{C}-)$. 
MS $(70 \mathrm{eV}, \mathrm{EI}): \mathrm{m} / \mathrm{z}(\%)=146(70)[\mathrm{M}-\mathrm{COOEt}]^{+}, 219(53)[\mathrm{M}]^{+}, 127$ (24) [M-Ph$\mathrm{NH}-\mathrm{C}]^{+}, 174(17)[\mathrm{M}-\mathrm{OEt}]^{+}$.

$\mathrm{C}_{12} \mathrm{H}_{13} \mathrm{NO}_{3}(219.09)$

\subsection{4-Oxo-4-(phenylamino)-3-(prop-2-ynylamino)buttersäureethylester (202)}<smiles>C#CCNC(CC(=O)OCC)C(=O)Nc1ccccc1</smiles>

Zu einer Lösung von dem Ester 200 (100 mg, 0.46 mmol, 1.0 Äq.) in $t \mathrm{BuOH}(1.2 \mathrm{~mL}$, $2.6 \mathrm{~mL}$ pro $\mathrm{mmol})$ wurde bei Raumtemperatur Propargylamin $(0.14 \mathrm{~mL}, 1.38 \mathrm{mmol}$, 3.0 Äq.) gegeben und die Reaktionsmischung für $24 \mathrm{~h}$ bei $100^{\circ} \mathrm{C}$ gerührt. Anschließend wurde auf Raumtemperatur abgekühlt und das Lösungsmittel im Vakuum entfernt. Der Rückstand wurde mit EE $(4.0 \mathrm{~mL})$ verdünnt und mit $10 \%$-iger $\mathrm{HCl}(3 \times 1.85 \mathrm{~mL})$ gewaschen. Die wässrige Phase wurde mit gesättigter $\mathrm{Na}_{2} \mathrm{CO}_{3}$ Lösung auf $\mathrm{pH} 8$ gebracht und mit EE $(3 \times 1.85 \mathrm{~mL})$ extrahiert. Die vereinigten organischen Phasen wurden mit $\mathrm{H}_{2} \mathrm{O}(5.0 \mathrm{~mL})$ gewaschen, über $\mathrm{MgSO}_{4}$ getrocknet und das Lösungsmittel im Vakuum entfernt. Das Rohprodukt wurde säulenchromatographisch (EE/P) gereinigt. Man erhielt die Titelverbindung (71.8 mg, $0.26 \mathrm{mmol}, 57 \%$ ) als orangenes Öl.

$\mathbf{R}_{\mathbf{f}}=0.27(\mathrm{EE} / \mathrm{P}=1: 4)$.

UV $\left(\mathrm{CH}_{3} \mathrm{CN}\right): \lambda_{\max }(\lg \varepsilon): 199.0 \mathrm{~nm}(1.118), 242.5$ (0.530).

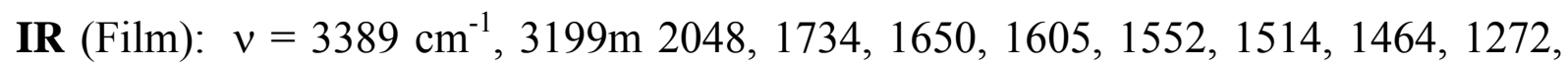
$1248,1178,1165,833$.

${ }^{1} \mathbf{H}-\mathbf{N M R}\left(300 \mathrm{MHz}, \mathrm{CDCl}_{3}\right): \delta=1.23\left(\mathrm{t}, 3 \mathrm{H}, \mathrm{CH}_{2} \mathrm{CH}_{3}, J=7.1 \mathrm{~Hz}\right), 2.24(\mathrm{t}, 1 \mathrm{H}$, Alkenyl-H, $J=2.4 \mathrm{~Hz}$ ), 2.81 (q, $\left.1 \mathrm{H}, 2-\mathrm{H}_{\mathrm{a}}, J=7.3,16.6 \mathrm{~Hz}\right), 2.94$ (q, $1 \mathrm{H}, 2-\mathrm{H}_{\mathrm{b}}, J=$ 4.2, $16.6 \mathrm{~Hz}), 3.45,3.55\left(\mathrm{dd}, 2 \mathrm{H}, \mathrm{CH}_{2} \mathrm{NH}, J=2.4,17.0 \mathrm{~Hz}\right), 3.65(\mathrm{dd}, 1 \mathrm{H}, 3-\mathrm{H}, J=$ 
4.3, $7.3 \mathrm{~Hz}), 4.14$ (q, $\left.2 \mathrm{H}, \mathrm{CH}_{2} \mathrm{CH}_{3}, J=7.1 \mathrm{~Hz}\right), 6.82-6.95$ (m, $\left.3 \mathrm{H}, \mathrm{Ph}-\mathrm{H}\right), 7.46$ (d, 2 $\mathrm{H}, \mathrm{Ph}-\mathrm{H}, J=8.9 \mathrm{~Hz}), 9.13\left(\mathrm{~s}_{\mathrm{b}}, 1 \mathrm{H}, \mathrm{NH}\right)$.

MS $\left(\mathrm{DCI}-\mathrm{NH}_{3}\right): m / z(\%)=274(100)[\mathrm{M}+1]^{+}$.

$\mathbf{C}_{15} \mathbf{H}_{18} \mathbf{N}_{2} \mathbf{O}_{3}(274.13)$

\subsection{3-(Benzylosycarbonyl)(prop-2-ynyl)amino)-4-oxo-4-}

(Phenylamino)buttersäureethylester (203)<smiles>C#CCN(CC)C(=O)CC(C(=O)Nc1ccccc1)[N+](=O)[O-]</smiles>

$\mathrm{Zu}$ einer Lösung von dem Ester 202 (65 mg, 0.24 mmol, 1.0 Äq.), Triethylamin (71.8 mg, $0.1 \mathrm{~mL}, 0.71 \mathrm{mmol}, 3.0$ Äq.) und katalytischen Mengen von DMAP in $\mathrm{CH}_{2} \mathrm{Cl}_{2}(0.5 \mathrm{~mL})$ wurde bei $0{ }^{\circ} \mathrm{C}$ eine Lösung von Chlorameisensäurebenzylester (0.04 mL, $0.29 \mathrm{mmol}, \quad 1.2$ Äq.) in $\mathrm{CH}_{2} \mathrm{Cl}_{2} \quad(0.25 \mathrm{~mL})$ zugegeben und die Reaktionsmischung für $12 \mathrm{~h}$ bei Raumtemperatur gerührt. Man wusch mit Wasser (10 mL), 2 N Salzsäure (10 mL), Wasser (10 mL), ges. $\mathrm{Na}_{2} \mathrm{CO}_{3}$-Lsg. (10 mL) und ges. NaCl-Lsg. $(10 \mathrm{~mL})$, trocknete die organische Phase über $\mathrm{Na}_{2} \mathrm{SO}_{4}$ und entfernte das Lösungsmittel im Vakuum. Man erhielt die Titelverbindung (85.2 mg, $0.21 \mathrm{mmol}$, $87 \%$ ) als ein orangenes, klebriges Öl.

$\mathbf{R}_{\mathbf{f}}=0.45(\mathrm{EE} / \mathrm{P}=1: 2)$.

$\mathbf{C}_{23} \mathbf{H}_{24} \mathbf{N}_{2} \mathbf{O}_{5}(408.17)$ 


\subsection{3-Aminoprapansäureessigester (208)}<smiles>CCOC(=O)CCN</smiles>

$\mathrm{Zu}$ einer rührenden Lösung von $\beta$-Alanin (89 mg, $1.0 \mathrm{mmol}, 1.0$ Äq.), 2,2Dimethoxypropan (1.6 mL, $1.6 \mathrm{~mL}$ pro mmol Amin) und Ethanol (0.6 mL, $0.6 \mathrm{~mL}$ pro mmol Amin) wurde TMSCl (0.013 mL, $0.1 \mathrm{mmol}, 0.1$ Äq.) gegeben und die Reaktionsmischung für $24 \mathrm{~h}$ bei Raumtemperatur gerührt. Das ausgefallene Hydrochlorid wurde abgenutscht, mit kaltem Diethylether gewaschen, in ges. $\mathrm{NaHCO}_{3}$-Lsg. (10 mL) aufgenommen und die Suspension so lange kräftig gerührt, bis sich der Feststoff vollständig aufgelöst hatte. Die wässrige Phase wurde mit Ether $(4 \times$ $20 \mathrm{~mL}$ ) extrahiert und die vereinigten organischen Phasen über $\mathrm{Na}_{2} \mathrm{SO}_{4}$ getrocknet. Das Lösungsmittel wurde im Vakuum entfernt und man erhielt die Titelverbindung (117 mg, $1.0 \mathrm{mmol}, 100 \%$ ) als ein farbloses Öl.

${ }^{1} \mathbf{H}-\mathbf{N M R}\left(300 \mathrm{MHz}, \mathrm{CDCl}_{3}\right): \delta=1.30\left(\mathrm{t}, 3 \mathrm{H}, \mathrm{CH}_{2} \mathrm{CH}_{3}, J=6.1 \mathrm{~Hz}\right), 2.01\left(\mathrm{~s}_{\mathrm{b}}, 1 \mathrm{H}\right.$, $\mathrm{NH}), 2.51\left(\mathrm{t}, 2 \mathrm{H}, 1-\mathrm{H}_{2}, J=4.8 \mathrm{~Hz}\right), 3.04\left(\mathrm{t}, 2 \mathrm{H}, 2-\mathrm{H}_{2}, J=4.8 \mathrm{~Hz}\right), 4.12(\mathrm{t}, 2 \mathrm{H}$, $\mathrm{CH}_{2} \mathrm{CH}_{3}, J=6.1 \mathrm{~Hz}$ ).

${ }^{13}$ C-NMR (50.3 MHz, $\left.\mathrm{CDCl}_{3}\right): \delta=14.11\left(\mathrm{CH}_{2} \mathrm{CH}_{3}\right), 38.30(\mathrm{C}-2), 38.99(\mathrm{C}-3), 61.32$ $\left(\mathrm{CH}_{2} \mathrm{CH}_{3}\right), 173.47(\mathrm{C}-1)$.

$\mathbf{C}_{5} \mathbf{H}_{11} \mathrm{NO}_{2}(117.08)$ 


\section{ANHANG}

\section{Verwendete Abkürzungen und Akronyme}

\begin{tabular}{|c|c|}
\hline AAV & Allgemeine Arbeitsvorschrift \\
\hline Abb. & Abbildung \\
\hline Ac & Acetyl \\
\hline$\ddot{A} q$ & Äquivalente \\
\hline APT & attached proton test \\
\hline $\mathrm{Ar}$ & Aryl \\
\hline Ber. & Berechnet \\
\hline Boc & tert-Butoxycarbonyl \\
\hline $\mathrm{Bn}$ & Benzyl \\
\hline $\mathrm{Bu}$ & Butyl \\
\hline $\mathrm{BuLi}$ & Butyllithium \\
\hline $\mathrm{Bz}$ & Benzyloxy \\
\hline bzw. & beziehungsweise \\
\hline $\mathrm{Cbz}$ & Benzyloxycarbonyl \\
\hline $\mathrm{d}$ & Tage \\
\hline $\mathrm{DC}$ & Dünnschichtchromatographie \\
\hline DCI & Direkte Chemische Ionisation \\
\hline DIBAL & Diisobutylaluminiumhydrid \\
\hline DMAP & $N, N$-Dimethylaminopyridin \\
\hline $\mathrm{DMF}$ & Dimethylformamid \\
\hline DMSO & Dimethylsulfoxid \\
\hline EDDA & Ethylendiamindiacetat \\
\hline ee & Enantiomerenüberschuss (enantiomeric excess) \\
\hline $\mathrm{EE}$ & Essigester \\
\hline EI & Elektronenstoß-Ionisation \\
\hline
\end{tabular}




\begin{tabular}{|c|c|}
\hline ESI & Elektrospray-Ionisation \\
\hline Et & Ethyl \\
\hline $\mathrm{Et}_{2} \mathrm{O}$ & Diethylether \\
\hline EtOH & Ethanol \\
\hline Gef. & Gefunden \\
\hline $\mathrm{h}$ & Stunde \\
\hline HOAc & Essigsäure \\
\hline HOMO & Highest Occupied Molecular Orbital \\
\hline HPLC & High-Pressure Liquid Chromatography \\
\hline HRMS & Hochaufgelöste Massenspektroskopie \\
\hline $\mathrm{Hz}$ & Hertz \\
\hline I & Intensität \\
\hline$i$ & iso \\
\hline IR & Infrarotspektroskopie \\
\hline konz. & Konzentriert \\
\hline 1 & flüssig (liquid) \\
\hline $\mathrm{L}$ & Ligand / Liter \\
\hline LDA & Lithiumdiisopropylamin \\
\hline Lsg. & Lösung \\
\hline LUMO & Lowest Unoccupied Molecular Orbital \\
\hline$m$ & meta \\
\hline M & Molar \\
\hline $\mathrm{Me}$ & Methyl \\
\hline $\mathrm{MeCN}$ & Acetonitril \\
\hline $\mathrm{MeOH}$ & Methanol \\
\hline $\min$ & Minute \\
\hline $\mathrm{MO}$ & Molekülorbital \\
\hline MS & Massenspektrometrie \\
\hline MS $4 \AA$ & Molsieb $4 \AA$ \\
\hline$n \mathrm{BuLi}$ & n-Buthyllithium \\
\hline NMO & $N$-Methylmorpholin- $N$-oxid \\
\hline
\end{tabular}




\begin{tabular}{|c|c|}
\hline NMR & nuclear magnetic resonance \\
\hline $\mathrm{Nu}$ & Nucleophil \\
\hline$o$ & ortho \\
\hline $\mathrm{OMe}$ & Methoxy \\
\hline$p$ & para \\
\hline PE & $n$-Pentan \\
\hline PG & Schutzgruppe \\
\hline $\mathrm{Ph}$ & Phenyl \\
\hline$p$-TsOH & $p$-Toluolsulfonsäure \\
\hline quant & quantitativ \\
\hline $\mathrm{R}_{\mathrm{f}}$ & Retentionswert bei Dünnschichtchromatographie \\
\hline RT & Zimmertemperatur (ca. $20-25^{\circ} \mathrm{C}$ ) \\
\hline $\mathrm{SC}$ & Säulenchromatographie \\
\hline Sdp. & Siedepunkt \\
\hline Smp. & Schmelzpunkt \\
\hline$t$ & tert \\
\hline TBAF & Tetrabutylammoniumfluorid \\
\hline$t-\mathrm{BuOH}$ & $t$-Buthanol \\
\hline TBS & tert-Butyldimethylsilyl \\
\hline tert & tertiär \\
\hline THF & Tetrahydrofuran \\
\hline TiPS & Triisopropylsilyl \\
\hline TMOF & Trimethylorthoformiat \\
\hline TMS & Trimethylsilyl \\
\hline Tos & $p$-Toluolsulfonyl \\
\hline UV & Ultraviolettspektroskopie \\
\hline vgl. & vergleiche \\
\hline VSS & Vanillin-Schwefelsäure \\
\hline $\mathrm{X}$ & Halogen \\
\hline z. B. & zum Beispiel \\
\hline
\end{tabular}




\section{Literaturverzeichnis}

[1] F. Wöhler, Ann. Phys. Chem.1828, 12, 253.

[2] J. Pietruszka, Nachr. Chem. Tech. Lab. 1998, 46, 963-971.

[3] S. Christensen, Newsweek 05.08.1991, 118, 1191.

[4] a) K. C. Nicolaou, W.-M. Dai, R. K. Guy, Angew. Chem. 1994, 106, 38-69; b)

L. Wessjohann, Angew. Chem. 1994, 106, 1011-1013; c) K. C. Nicolaou, Z. Yang, J. J. Liu, H. Ueno, P. G. Nantermet, R. K. Guy, C. F. Claiborne, J. Renaud, E. A. Couladouros, K. Paulvannan, E. J. Sørensen, Nature 1994, 367, 630-634; d) R. A. Holton, C. Somoza, H.-B. Kim, F. Liang, R. J. Biediger, P. D. Boatmen, M. Shindo, C.C. Smith, S. Kim, H. Nadizadeh, V. Suzuki, C. Tao, P. Vu, S. Tang, P. Zhang, K. K.Murthi, L. N. Gentile, J. H. Liu, J. Am. Chem. Soc. 1994, 116, 1597-1598; e) R. A. Holton, H.-B. Kim, C. Somoza, F. Liang, R. J. Biediger, P. D. Boatmen, M. Shindo, C. C. Smith, S. Kim. H. Nadizadeh, Y. Suzuki, C. Tao, P. Vu, S. Tang, P. Zhang, K. K. Murthi, L. N. Gentile, J. H. Liu, J. Am. Chem. Soc. 1994, 116, 1599-1600; f) J. J. Masters, J. T. Link, L. B. Snyder, W. B. Young, S. J. Danishefsky, Angew. Chem. 1995, 107, 1886-1888; g) K. C. Nicolaou, R. K. Guy, Angew. Chem. 1995, 107, 2247-2259; h) K. C. Nicolaou, P. G. Nantermet, H. Ueno, R. K. Guy, E. A. Couladouros, E. J. Sørensen, J. Am. Chem. Soc. 1995, 117, 624-633; i) K. C. Nicolaou, J.-J. Liu, Z. Yang, H. Ueno, E. J. Sørensen, C. F. Claiborne, R. K. Guy, C.-

K. Hwang, M. Nakada, P. G. Nantermet, J. Am. Chem. Soc. 1995, 117, 634644; j) K. C. Nicolaou, Z. Yang, J.-J. Liu, P. G. Nantermet, C. F. Claiborne, J. Renaud, R. K. Guy, K. Shibayama, J. Am. Chem. Soc. 1995, 117, 645-652; k) K. C. Nicolaou, H.Ueno, J.-J. Liu, P. G. Nantermet, Z. Yang, J. Renaud, K. Paulvannan, R. Chadha, J. Am. Chem. Soc. 1995, 117, 653-659; 1) S. J. Danishefsky, J. J. Masters, W. B. Young, J. T. Link, L. B. Snyder, T. V. Magee, D. K. Jung, R. C. A. Isaacs, W. G. Bornmann, C. A. Alaimao, C. A. Coburn, M. J. Di Grandi, J. Am. Chem. Soc. 1996, 118, 2843-2859; m) P. A. Wender, N. F. Badham, S. P. Conway, P. E. Floreancig, T. E. Glass, C. Gränicher, J. B. Houze, J. Jänichen, D. Lee, D. G. Marquess, P. L. McGrane, W. Meng, T. P. Mucciaro, M. Mühlebach, M. G. Natchus, H. Paulsen, D. B. Rawlins, J. 
Satkofsky, A. J. Shuker, J. C. Sutton, R. E. Taylor, K. Tomooka, J. Am. Chem. Soc. 1997, 119, 2755-2756; n) P. A. Wender, N. F. Badham, S. P. Conway, P. E. Floreancig, T. E. Glass, J. B. Houze, N. E. Krauss, D. Lee, D. G. Marquess, P. L. McGrane, W. Meng, M. G. Natchus, A. J. Shuker, J. C. Sutton, R. E. Taylor, J. Am. Chem. Soc. 1997, 119, 2757-2758.

a) R. W. Armstrong, J.-M. Beau, S. H. Cheon, W. J. Christ, H. Fujioka, W.-H. Ham, L. D. Hawkins, H. Jin, S. H. Kang, Y. Kishi, M. J. Martinelli, W. W. McWhorter, M. Mizuno, M. Nakata, A. E. Stutz, F. X. Talamas, M. Taniguchi, J. A. Tino, K. Udea, J. Uenishi, J. B. White, M. Yonaga, J. Am. Chem. Soc. 1989, 111, 7525-7530; b) R. W. Armstrong, J.-M. Beau, S. H. Cheon, W. J. Christ, H. Fujioka, W.-H. Ham, L. D. Hawkins, H. Jin, S. H. Kang, Y. Kishi, M. J. Martinelli, W. W. McWhorter, M. Mizuno, M. Nakata, A. E. Stutz, F. X. Talamas, M. Taniguchi, J. A. Tino, K. Udea, J. Uenishi, J. B. White, M. Yonaga, J. Am. Chem. Soc. 1989, 111, 7530-7533; c) Y. Kishi, Pure Appl. Chem. 1989, 61, 313-324; d) E. M. Suh, Y. Kishi, J. Am. Chem. Soc. 1994, $116,11205-11206$.

[6] a) K. C. Nicolaou, E. A. Theodorakis, F. P. J. T. Rutjes, J. Tiebes, M. Sato, E. Untersteller, X.-Y. Xiao, J. Am. Chem. Soc. 1995, 117, 1171-1172; b) K. C. Nicolaou, F. P. J. T. Rutjes, E. A. Theodorakis, J. Tiebes, M. Sato, E. Untersteller, J. Am. Chem. Soc. 1995, 117, 1173-1174; c) K. C. Nicolaou, F. P. J. T. Rutjes, E. A. Theodorakis, J. Tiebes, M. Sato, E. Untersteller, J. Am. Chem. Soc. 1995, 117, 10252-10263.

[7] a) E. J. Corey, P. B. Hopkins, S. Kim, S. Yoo, K. P. Nambiar, J. R. Falck, J. Am. Chem. Soc. 1979, 101, 7131-7134; b) R. B. Woodward, E. Logusch, K. P. Nambiar, K. Sakan, D. E. Ward, J. Am. Chem. Soc. 1981, 103, 3215-3217; c) G. Stork, S. D. Rychnovsky, J. Am. Chem. Soc. 1987, 109, 1564-1565; c) G. Stork, S. D. Rychnovsky, J. Am. Chem. Soc. 1987, 109, 1565-1567; d) G. Stork, S. D. Rychnovsky, Pure Appl. Chem. 1986, 58, 767-772; e) R. Stürmer, K. Ritter, R. W. Hoffmann, Angew. Chem. 1993, 105, 112-114.

a) D. A. Evans, M. R. Wood, B. W. Trotter, T. I. Richardson, J. C. Barrow, J. L. Katz, Angew. Chem. 1998, 110, 2864-2868; b) D. A. Evans, C. J. Dinsmore, P. S. Watson, M. R. Wood, T. I. Richardson, B. W. Trotter, J. L. Katz, Angew. 
Chem. 1998, 110, 2868-2872; c) K. C. Nicolaou, S. Natarajan, H. Li, N. F. Jain, R. Hughes, M. E. Solomon, J. M. Ramanjulu, C. N. C. Boddy, M. Takayanagi, Angew. Chem. 1998, 110, 2872-2878; d) K. C. Nicolaou, N. F. Jain, S. Natarajan, R. Hughes, M. E. Solomon, H. Li, J. M. Ramanjulu, M. Takayanagi, A. E. Koumbis, T. Bando, Angew. Chem. 1998, 110, 2879-2881; e) K. C. Nicolaou, M. Takayanagi, N. F. Jain, S. Natarajan, A. E. Koumbis, T. Bando, J. M. Ramanjulu, Angew. Chem. 1998, 110, 2881-2883; f) J. Zhang, K. Burgess, Angew. Chem. 1999, 111, 666-669; g) K. C. Nicolaou, C. N. C. Boddy, S. Bräse, N. Winssinger, Angew. Chem. 1999, 111, 2230- 2287; h) K. C. Nicolaou, H. Li, C. N. C. Boddy, J. M. Ramanjulu, T.-Y. Yue, S. Natarajan, X.J. Chu, S. Bräse, F. Rübsam, Chem. Eur. J. 1999, 5, 2584-2601; i) K. C. Nicolaou, C. N. C. Boddy, H. Li, A. E. Koumbis, R. Hughes, S. Natarajan, N. F. Jain, J. M. Ramanjulu, S. Bräse, M. E. Solomon, Chem. Eur. J. 1999, 5, 2602-2621; j) K. C. Nicolaou, A. E. Koumbis, M. Takayanagi, S. Natarajan, N. F. Jain, T. Bando, H. Li, R. Hughes, Chem. Eur. J. 1999, 5, 2622-2647; k) K. C. Nicolaou, H. J. Mitchell, N. F. Jain, T. Bando, R. Hughes, N. Winssinger, S. Natarajan, A. E. Koumbis, Chem. Eur. J. 1999, 5, 2648-2667; 1) D. L. Boger, S. Miyazaki, S. H. Kim, J. H. Wu, O. Loiseleur, S. L. Castle, J. Am. Chem. Soc. 1999, 121, 3226-3227.

a) R. A. Holton, C. Somoza, K. B. Kim, F. Liang, R. J. Biedinger, P. D. Boatman, M. Shindo, C. C. Smith, S. Kim, H. Nadizadeh, Y. Suzuki, C.tao, P. Vu, S. Tang, P. Zhang, K. K. Murthi, L. N. Gentile, J. H. Liu, J. Am. Chem. Soc. 1994, 116, 1597-1598; b) R. A. Holton, C. Somoza, K. B. Kim, F. Liang, R. J. Biedinger, P. D. Boatman, M. Shindo, C. C. Smith, S. Kim, H. Nadizadeh, Y. Suzuki, C. Tao, P. Vu, S. Tang, P. Zhang, K. K. Murthi, L. N. Gentile, J. H. Liu, J. Am. Chem. Soc. 1994, 116, 1599-1600.

[10] a) H.-J. Böhm, G. Klebe, H. Kubinyi, Wirkstoffdesign, Spektrum, Heidelberg, 1996; b) D. Lednicer, Strategies for Organic Drug Synthesis and Design, Wiley, New York, 1998.

[11] a) L. F. Tietze, U. Beifuss, Angew. Chem. 1993, 105, 137-170; b) L. F. Tietze, Chem. Ind. 1995, 453-457; c) L. F. Tietze, Chem. Rev. 1996, 96, 115-136; d) L. F. Tietze, Nachr. Chem. Lab. 1997, 45, 1181-1187; e) L. F.Tietze, F. 
Haunert in: Stimulating Concepts in Chemistry, F. Vögtle, J. F. Stoddart, M. Shibasaki (Hrsg.) 2000, Wiley-VCH, Weinheim, S. 40.

[12] B. M. Trost, Angew. Chem. 1995, 107, 285-307.

[13] W. A. Herrmann, B. Cornils, Applied Homogeneous Catalysis with Organometallic Compounds, VCH, Weinheim, 1996.

[14] A. Hegstrom, D. K. Kondepudi, Spektrum Wiss. 1990, 3, 56.

[15] a) J. W. Scott, Top. Stereochem. 1989, 19, 209; b) E. Mutschler, Arzneimittelwirkungen, Wissenschaftliche Verlagsgesellschaft, Stuttgart, 1991.

[16] a) E. J. Corey, X.-M. Cheng, The Logic of Chemical Synthesis, Wiley, New York, 1989; b) K. C. Nicolaou, E. J. Sørensen, Classics in Total Synthesis, VCH, Weinheim, 1996.

[17] a) L. A. Thompson, J. A. Ellmann, Chem. Rev. 1996, 96, 555-600. b) S. R. Wilson, A. W. Czarnik (Hrsg.), Combinatorial Chemistry, Wiley, New York, 1997.

[18] W. Meissner, 'Über ein neues Pflanzenalkali (Alkaloid)', J. Chemie und Physik (Schweiger) 1819, 25, 379.

[19] http://pharm1.pharmazie.uni-greifswald.de/systematik/ergaenz/alkaloid.htm.

[20] S. W. Pelletier, Alkaloids-Chemical and Biologically Properties, Vol. 1. S. 1, Siley, New York 1983.

[21] K. B. G. Torssell, Natural Product Chemistry, Apotekarsocieteten, Stockholm 1997, S. 348.

[22] W. A. Creasey in The Chemistry of Heterocyclic Compounds, A. Weisenberger und E. C. Taylor, Vol. 25, Part 4, S. 783, Wiley, New York 1983.

[23] M. Seefelder, Opium, eine Kulturgeschichte, ecomed Verlagsgesellschaft, Landsberg 1996.

[24] D. S. Siegler, The Alkaloids, 1977, 16, R.H.F. Manske (Hrsg.), Academic Press, New York.

[25] http://heilpflanzen.m-ww.de/zzwirkstoffe/alkaloide.html.

[26] A. Schäfer, H. Benz, W. Friedler, A. Guggisberg, S. Bienz, M. Hesse, 'Polyamine Toxins from Spider and Wasps', The Alkaloids 1994, 45, 1. 
[27] R. K. Hill, 'Pyrrolidine, Piperidine, Pyridine and Imidazole Alkaloids', in S. W. Pelletrier (Ed.), Chemistry of the Alkaloids, Van Nostrand, New York 1970, 385.

[28] a) G. M. Strunz, J. A. Findlay, 'Pyridine and Piperidine Alkaloids', The Alkaloids 1985, 26, 89; b) W. A. Ayer, T. E. Habgood, 'The Pyridine Alkaloids', The Alkaloids 1968, 11, 459; c) D. Gross, 'Naturstoffe mit Pyridinstruktur und ihre Biosynthese', Fortschr. Chem. Organ. Naturstoffe 1970, 28, 109.

[29] a) H.-P. Ros, R. Kyburz, N. W. Preston, R. T. Gallagher, I. R. C. Bick, M. Hesse, 'The Structure of the Alkaloid Peduncularine', Helv. Chim. Acta 1985, 24, 113;b) H.-J. Borschberg, 'Aristotelia Alkaloids', The Alkaloids 1996, 48, 192.

[30] Allgemeine Übersicht: a) A. R. Battersby, 'Recent Researches on Indole Alkaloids', Pure Appl. Chem 1963, 6, 471; b) L. Marion, 'The Indole Alkaloids', The Alkaloids 1952, 2, 369; c) N. Neuss, 'Indole Alkaloids' in S. W. Pelletier (Ed.), Chemistry of the Alkaloids, Van Nostrand, New York 1970, 213; d) J. E. Saxton, 'The Indole Alkaloids', The Alkaloids 1960, 7, 1; e) J. E. Saxton, 'The Indole Alkaloids excluding Harmine and Strychnine', Quart. Rev. 1956, 10, 108; f) W. I. Taylor, Indole Alkaloids, an Introduction to the Enamine Chemistry of Natural Products, Pergamon Press, Inc., New York 1966.

[31] a) C. Szántay, G. Blaskó, K. Honty, G. Dörnyei, 'Corynantheine, Yohimbine and Related Alkaloids', The Alkaloids 1986, 27, 131; b) E. Schlittler, 'Rauwolfia Alkaloids with Special Reference to the Chemistry of Reserpine', The Alkaloids 1965, 8, 287; c) R. T: Brown in The Chemistry of Heterocyclic Compounds, J. E. Saxton (Ed.), John Wiley and Sons, New York 1983, Vol. 25 , S. Pt. 4, S. 147-199; d) A. Chatterjee, Pure Appl. Chem. 1986, 58, 685; e) S. Szantay, Pure Appl. Chem. 1990, 62, 1299; f) E. W. Baxter, P. S. Mariano in Alkaloids: Chemical and Biological Perspectives, S. Q. Pelletier (Ed.), Springer-Verlag, New York 1992, Vol. 8, 197.

[32] a) R. E. Woodson, H. W. Youngken, E. Schlittler, J. A. Schneider in Rauwolfia: Botany, Pharmacognosy, Chemistry and Pharmacology, Little Brown and Co., Boston 1957; b) S. C. Bergmeier, P. P. Seth, J. Org. Chem. 1999, 64, 3237. 
[33] J. A. Scott, F. T. Crews, J. Pharmacol. Exp. Ther. 1983, 224, 640.

[34] a) G. Habermehl, P. E. Hammann, Naturstoffchemie: Eine Einführung, Springer-Verlag, New York 1992; b) G. A. Cordell, Introduction to Alkaloids, A Biogenetic Approach, Wiley-Interscience, New York 1981, 574.

[35] J. F. Treimer, M. H. Zenk, Eur. J. Biochem. 1979, 101, 225-233.

[36] T. Hemscheid, M. H. Zenk, FEBS Lett. 1980, 110, 187-191.

[37] R. T: Brown in The Chemistry of Heterocyclic Compounds, A. Weisenberger, E. C. Taylor (Hrsg.), Vol. 25, Part 4, Wiley, New York 1983.

[38] J. Aubé, S. Ghosh, M. Tanol, J. Am. Chem. Soc. 1994, 116, 9009.

[39] R. B. Woodward, F. E. Bader, H. Bickel, A. J. Frey, R. W. Kierstead, Tetrahedron 1958, 2, 1-57.

[40] a) E. E. van Tamelen, M. Shamma, A. W. Burgstahler, J. Wolinsky, R. Tamm, P. E. Aldrich J. Am. Chem. Soc. 1958, 80, 5006; b) E. E. van Tamelen, M. Shamma, A. W. Burgstahler, J. Wolinsky, R. Tamm, P. E. Aldrich J. Am. Chem. Soc. 1969, 91, 7315.

[41] a) M. E. Kuehne, R. S. Muth, J. Org. Chem. 1991, 56, 2701-2712; b) N. Aimi, E. Yamanaka, J. Endo, S. Sakai, J. Haginiwa, Tetrahedron 1973, 29, 20152021; c) K. Okamura, S.-I. Yamada Chem. Pharm. Bull. 1978, 26, 2305-2311; d) T. Kametani, N. Takgi, N. Kanaya, T. Honda, K. Fukumoto, J. Heterocycl. Chem. 1982, 19, 1217-1219; e) M. Isobe, N.Fukami, T.Goto, Chem. Lett. 1985, 71-74; f) R. Riva, L. Banfi, B. Danieli, G. Guanti, G. Lesma, G. Palmisano, J. Chem. Soc., Chem. Commun. 1987, 299-300; g) A. I. Meyers, D. B. Miller, F. H. White, J. Am. Chem. Soc. 1988, 110, 4778-4787; h) J. Aubé, Tetrahedron Lett. 1988, 29, 4509-4512; i) D. H. Hua, S. N. Bharathi, F. Takusagawa, A. Tsujimoto, J. A. Panangadan, M.-H. Hung, A. A. Bravo, A. M. Epelding, J. Org. Chem. 1989, 54, 5659-5662; j) J. Aubé, Y. Wang, M. Hammond, M. Tanol, F. Takusagawa, D. Vander Velde, J. Am. Chem. Soc. 1990, 112, 48794891; k) A. I. Meyers, T. K. Highsmith, T. P. Buonora, J.Org. Chem. 1991, 56, 2960-2964; 1)D. H. Hua, S. N. Bharathi, J. A. K. Panangadan, A. Tsujimoto, J. Org. Chem. 1991, 56, 6998-7007; m) Y. Hirai, T. Terada, T. Yamazaki, T. Momose, J. Chem. Soc., Perkin Trans. 1 1992, 517-524. 
[42] G. Blaskó, H. Knight, K. Honty, C. Szántay, Liebigs Ann. Chem. 1986, 655663.

[43] Y. Hirai, T. Terade, Y. Okaji, T. Yamazaki, T. Momose, Tetrahedron Lett. 1990, 31, 4755-4756.

[44] H. T. Openshaw, 'Quinoline Alkaloids, other than those of Chinchona', The Alkaloids 1953, 3, 65; ibid. 1960, 7, 229; ibid. 1967, 9, 223.

[45] a) M. J. Luzzio, J. M. Bestermann, D. L. Emerson, M. G. Evans, K. Lackey, P. L. Leitner, G. McIntyre, B. Morton, P. L. Myers, M. Peel, J. M. Sisco, D. D. Sternbach, W. Q. Tong, A. Truesdale, D. E. Uehling, A. Vuong, J. Yates, J. Med. Chem. 1995, 38, 395; b) D. E. Uehling, S. S: Nanthakumar, D. Croom, D. L. Emerson, P. L. Leitner, M. J. Luzzio, G. McIntyre, B. Morton, S. Profeta, J. M. Sisco, D. D. Sternbach, W. Q. Tong, A. Vuong, J. Yates, J. M. Bestermann, J. Med. Chem. 1995, 38, 1106.

[46] a) M. E. Wall, M. C. Wani in The Chemistry of Heterocyclic Compounds: Monoterpenoid Indole Alkaloids, J. E. Saxton (Ed.), John Wiley and Sons: Chichester, UK 1994, Vol. 25 (4), 689-713; b) M. E. Wall, M. C. Wani, A. W. Nicholas, G. Manikuma, C. Tele, L. Moore, A. Truesdale, P. Leitner, J. M. Besterman, J. Med. Chem.1993, 36, 2689; c) M. Suffness, G. A. Cordell in The Alkaloids, A. Brossi (Ed), Academic Press, Orlando, FL 1985, Vol. 25, ch. 1, 75; d) J. C. Cai, C. R. Hutchinson, Chem. Heterocycl. Compd. 1983, 25, 753; e) C. R: Hutchinson, Tetrahedron 1981, 37, 1047; f) J. C. Cai, C. R. Hutchinson, The Alkaloids: Chemistry and Pharmacology, A. Brossi (Ed), Academic Press, Orlando, FL 1983, Vol. 21, 101; g) A. G. Schultz, Chem. Rev. 1973, 73, 385; h) M. Shamma, Experientia, 1968, 24, 107.

[47] a) M. A. Ciufolini, F. Roschangar, Tetrahedron 1997, Vol. 53, 32; b) R. J. Anderson, G. B. Raolji, A. Kanazawa, A. E. Greene, Org. Lett. 2005, Vol. 7, 2989-2991.

[48] M. E. Wall, M. C. Wani, C. E. Cook, K. H. Palmer, A. T. MacPhail, G. A: Sim, J. Am. Chem. Soc. 1966, 88, 3888-3890.

[49] a) W. Du, Tetrahedron 2003, 59, 8649-8687; b) A. Lansiaux, C. Bailly, Bull. Cancer 2003, 90, 239-245; c) Camptothecins: New Anticancer Agents, M. Potmesil, H. Pinedo (Ed), CRC Press: Boca Raton 1995; d) S. Sawada, T. 
Yokokura, T. Miyakasa, Curr. Pharm. Des. 1995, 1, 113-132; e) M. Torck, M. Pinkas, J. Pharm. Belg. 1996, 51, 200-207; f) C. R. Hutchinson, Tetrahedron 1981, 37, 1047-1065.

[50] Y. H. Hsiang, R. Hertzberg, S. Hecht, L. F: Liu, J.Biol. Chem. 1985, 260, 14873-14878.

[51] W. D. Kingsbury, J. C. Boehm, D. R. Jakas, K. G. Holden, S. M. Hecht, G. Gallagher, M. J. Caranfa, F. L. McCabe, L. F. Faucette, R. K. Johnson, R. P. Hertzberg, J. Med. Chem. 1991, 34, 98-107.

[52] a) S. Negoro, M. Fukuoka, N. Masuda, M. Takada, Y. Kusunoki, K. Matsui, N. Takifuji, S. Kudoh, H. Niitani, T. Taguchi, J. Natl. Cancer Inst. 1991, 83, 11641168; b) Y. Kawato, M. Aonuma, Y. Hirota, H. Kuga, K. Sato, Cancer Res. 1991, 51, 4187-4191.

[53] a) G. M. Cragg, D. J. Newman, J. Nat. Prod. 2004, 67, 232-244; b) M. Butler, Nat. Prod. Rep. 2005, 22, 162-195.

[54] O. Diels, K. Alder, Justus Liebigs Ann. Chem. 1928, 44, 98-122.

[55] umfassender Aufsatz: K. C. Nicolauo, S. A. Snyder, T. Montagnon, G. E. Vassilikogiannakis, Angew. Chem. 2002, 114, 1742-1773; Angew. Chem. Int. Ed. 2002, 41, 1668-1698.

[56] K.C. Nicolaou, Scott A. Snyder, T. Montagnon, G. E. Vassilikogiannakis, Angew. Chem. 2002, 114, 1742-1773.

[57] a) K. C. Nicolaou, J.-K. Jung, W. H. Yoon, Y. He, Y.-L- Zhong, P. S. Baran, Angew. Chem. 2000, 112, 1899-1902; Angew. Chem. Int. Ed. 2000, 39,1829-1832; b) K.C. Nicolaou, J. Jung, W. H. Yoon, K. C. Fong, H.-S. Choi, Y. He, Y.-L. Zhong, P. S. Baran J. Am. Chem. Soc. 2002, 124, 2183-2189; c) K. C. Nicolaou, P. S. Baran, Y.-L. Zhong, K. C. Fong, H.-S. Choi, J. Am. Chem. Soc. 2002, 124, 2190-2201; d) K. C. Nicolaou, Y.-L. Zhong, P. S. Baran, J. Jung, H.-S. Choi, W. H. Yoon, J. Am. Chem. Soc. 2002, 124, 2202-2211.

[58] N. Waizumi, T. Itoh, T. Fukuyama, J. Am. Chem. Soc. 2000, 122, 7825-7826.

[59] M. E. Layton, C. A. Morales, M. D. Shair, J. Am. Chem. Soc. 2002, 124, 773775. 
[60] N. Fusetani, H. Nagata, H. Hirota, T. Tsuyuki, Tetrahedron Lett. 1989, 30, 7079-7082.

[61] Y. Seo, J.-R. Rho, K. W. cho, J. Shin, J. Nat. Prod. 1997, 60, 171-174.

[62] M. T. Crimmins, B. H. Brown, J. Am. Chem. Soc. 2004, 126, 10264-10266.

[63] a) P.-S. Sung, M.-C. Chen, Heterocycles 2002, 57, 1705-1715; b) P. Bernadelli, L.A. Paquette, Heterocycles 1998, 49, 531-556; c) A. Rodrîguez, Tetrahedron 1995, 51, 4571-4618; d) J. C. Coll, Chem. Rev. 1992, 92, 613-631.

[64] O. Kennard, D. G. Watson, L. Riva di Sanseverino, B. Tursch, R. Bosmans, C. Djerassi, Tetrahedron Lett. 1968, 9, 2879-2884.

[65] M. G. Charest, D. R. Siegel, A. G. Myers, J. Am. Chem. Soc. 2005, 127, 8292-9293.

[66] L. F. Tietze, G. Kettschau in Topics in Current Chemistry, Vol. 189, Springer, 1997, $1-120$.

[67] Z. Chen, J. Fan, A. S. Kende, J. Org. Chem. 2004, 69, 79-85.

[68] T. O. Larsen, K. Frydenvang, J. C. Frisvad, C. Christophersen, J. Nat. Prod. 1998, 61, 1154-1157.

[69] a) K. Alder, G. Stein, Angew. Chem. 1937, 50, 510-519; b) J. Sauer, Angew. Chem. 1967, 79, 76-94; Angew. Chem. Int. Ed. 1967, 6, 16-25.

[70] a) R. B. Woodward, R. Hoffmann, Die Erhaltung der Orbitalsymmetrie, Verlag Chemie, Weinheim 1970; b) R. B. Woodward, R. Hoffmann, Angew. Chem. 1969, 81, 797-869, Angew. Chem. Int. Ed. 1969, 8, 781-853.

[71] K. N. Houk, Y. Li, J. D. Evanseck, Angew. Chem. 1992, 104, 711-739; Angew. Chem. Int. Ed. 1992, 31, 682-708.

[72] J. Sauer, R. Sustmann, Angew. Chem. Int. Ed. 1980,19, 779-807; Angew. Chem. Int. Ed., 1990, 19, 779-807.

[73] a) L. F. Tietze, M. Bratz, R. Machinek, G. v. Kiedrowski, J. Org. Chem. 1987, 52, 1638-1640; b) L. F. Tietze, T. Brumby, S. Brand, M. Bratz, Chem. Ber. 1988, 121, 499-506.

[74] K. Fukui, Acc. Chem. Res. 1971, 4, 57-64.

[75] K. N. Houk, Acc. Cem. Res. 1975, 8, 361-396. 
[76] a) G. Klopman, J. Am. Chem. Soc. 1968, 90, 233-234; b) L. Salem, J. Am. Chem. Soc. 1968, 90, 543-552; c) L. Salem, J. Am. Chem. Soc. 1968, 90, $553-566$.

[77] R. Sustmann, R. Schubert, Tetrahedron Lett. 1972, 13, 2739-2742.

[78] G. Desimoni, P. P. Righetti, E. Selva, G. Tacconi, V. Rigante, M. Specchiraello, Tetrahedron 1977, 33, 2829-2830.

[79] a) K. N. Houk, J. Am. Chem. Soc. 1973, 95, 4092-4094; b) K. N. Houk, J. Sims, C. R. Watts, L. J. Luskus, J. Am. Chem. Soc. 1973, 95, 7301-7315.

[80] I. Flemming, Grenzorbitale und Reaktionen organischer Verbindungen, VCH, Weinheim, 1979.

[81] D. Ginsburg, Tetrahedron 1983, 39, 2095-2135.

[82] M. A. Fox, R. Cardona, N. J. Kiewiet, J. Org. Chem. 1987, 52, 1469-1474.

[83] J. I. Garcia, J. A. Mayornal, L. Salvatella, Acc. Chem. Res. 2000, 33, 658-664.

[84] a) L. F. Tietze, U. Beifuss, Angew. Chem. 1993, 105, 137-170; Angew. Chem. Int. Ed. 1993, 32, 131-163; b) L. F. Tietze, Chem. Rev. 1996, 96, 115-136; c) L. F. Tietze, F. Haunert in Stimulating Conceps in Chemistry, F. Vögtle, J. F. Stoddart, M. Shibasaki (Hrsg.), Wiley-VCH, Weinheim 2000; d) L. F. Tietze, A. Modi, Med. Res. Rev. 2000, 20, 304-322.

[85] a) E. J. Corey, P. R. Ortiz de Montellano, J. Am. Chem. Soc. 1966, 88, 47504751, b) E. J. Corey, S. C. Vergil, D. R. Liu, S. Sarshar, J. Am. Chem. Soc. 1992, 114, 1524-1527.

[86] D. A. Vosburg, C. D. Vanderwal, E. J. Sorensen, J. Am. Chem. Soc. 2002, 124, $4552-4553$.

[87] L. F. Tietze, J. Wichmann, Angew. Chem. 1992, 104, 1091-1092.

[88] a) L. F. Tietze, U. Beifuss, M. Ruther, J. Org. Chem. 1989, 54, 3120-3129, b) L. F. Tietze, U. Beifuss, Org. Synth. 1992, 71, 167-174.

[89] a) L. F. Tietze, J. Bachmann, J. Wichmann, Y. Zhou, T. Raschke, Liebigs Ann./Recueil 1997, 881-886, b) L. F. Tietze, J. Bachmann, J. Wichmann, O. Burkhardt, Synthesis 1994, 1185-1194, c) L. F. Tietze, J. Bachmann, W. Schul, Angew. Chem. 1988, 100, 983-985. 
[90] L. F. Tietze, U. Beifuss, J. Antel, G. M. Sheldrick, Angew. Chem. 1988, 100, 739-741.

[91] a) L. F. Tietze, N. Rackelmann in Multicomponent Reactions, 2004, J. Zhu, O. Binaymé (Hrgs.), Wiley-VCH, Weinheim, submitted; b) L. F. Tietze, N. Rackelmann, I. Müller, Chem.-Eur. J. 2004, in press; c) L. F. Tietze, N. Rackelmann, Z. Naturfarsch., B: Chem. Sci. 2004, submitted; d) L. F. Tietze, Y. Zhou, E. Töpken, Eur. J. Org. Chem. 2000, 2247-2252; e) L. F. Tietze, Y. Zhou, Angew. Chem. 1999, 111, 2076-2078; Angew. Chem. Int. Ed. 1999, 38, 2045-2047; f) L. F. Tietze, J. Bachmann, J. Wichmann, Y. Zhou, T. Raschke, Liebigs Ann./Receuil 1997, 881-886; g) L. F. Tietze, J. Bachmann, J. Wichmann, O. Burkhardt, Synthesis 1994, 1185-1194; h) L. F. Tietze, J. Bachmann, W. Schul, Angew. Chem. 1988, 100, 983-985.

[92] L. F. Tietze, U. Beifuss in Comprehensive Organic Synthesis 1991, B. M. Trost (Hrgs.), Pergamon Press, Oxford, Vol. 2, S.341.

[93] L. F. Tietze, A. Steinmetz, F. Balkenhohl, Bioorganic and Medicinal Chemistry Letters 1997, 7, 1303-1306.

[94] L. F. Tietze, G. Kettschau, J. A. Gewert, A. Schuffenhauer, Curr. Org. Chem. 1998, 2, 19-62.

[95] L. F. Tietze, N. Rackelmann, Pure Appl. Chem, 2004, Vol. 76, 1967-1983. 


\section{Danksagung}

Den Damen und Herren der NMR-Abteilung Carola Zolke, Matthias Decke, Andrea Godawa, Christiane Siebert, Reinhard Machinek und Ulrich Leonhardt danke ich vielmals für das schnelle Messen zahlreicher NMR-Spektren. Bei Frau Evelyn Pfeil möchte ich mich für dieAufnahme der IR- und UV-Spektren und die Ermittlung vieler spezifischer Drehwerte bedanken. Weiterhin gilt mein Dank Frau Gvörgvi Udvarnoki und Herrn Dr. Holm Frauendorf für die Aufnahme der Massenspektren und Herrn Olaf Senge für das Anfertigen der HPLC-Analysen. Dr. Sascha Hellkamp und Martina Pretor ist die Instandhaltung der EDV-Infrastruktur und Dr. Sören Hölsken, Dr. Holger Braun sowie Niels Böhnke die Verteilung der Materialien zu verdanken.

Meinen beiden Korrekturlesern Konrad und Julia danke ich sehr für die Ausdauer und Geduld, die sie für das Korrigieren dieser Arbeit aufgebracht haben. Insbesondere meiner kleinen Schwester Julia kann ich nicht genug danken, dass sie trotz der Lernphase für ihr zweites Jura-Staatsexamen und zusätzlich als Frau nicht vom Fach sich die Mühe gemacht und Zeit genommen hat, eine für sie eher dem chinesisch gleichende Arbeit durchzulesen. Damit hast du meine grenzenlose Hochachtung!

Auch wenn es nicht einfach gewesen ist, Timo als Sitznachbar im MathematikLeistungskurs in der Schule gehabt zu haben oder als Praktikumspartner während des Studiums, so kann ich mich doch sehr glücklich schätzen, in ihm einen wahren Freund gefunden zu haben. Timo danke ich daher für sehr viele tolle und lustige Momente während der Zeit in Göttingen und darüber hinaus.

Den größten Dank bin ich jedoch meinen Eltern, meiner Schwester Julia und Konrad verpflichtet. Dafür, dass meine Schwester sich auf das Abenteuer eingelassen hat, mit mir zusammenzuziehen und wir somit sechs aufregende und unvergessene Jahre gemeinsam erleben konnten mit allen Hoch und Tiefs, dafür, daß unsere Mutter uns immer mit bester Hausmannskost "über"-versorgt hat und unser Vater allzeit mit seinem Werkzeug zur Hilfe stand. Und vor allem dafür, daß Konrad mein Leben auf eine ganz besondere Weise bereichern konnte. 


\section{Lebenslauf}

\section{Persönliche Daten}

Name:

Geburtsdatum:

Geburtsort:

Eltern:

Staatsangehörigkeit:

Familienstand:

\section{Schulausbildung}

$1984-1985$

$1985-1986$

$1986-1988$

$1988-1997$

Juli 1997

\section{Studium}

Okt. 1997 - Juli 2003 Studium der Chemie an der Georg-August-Universität in

05. Juli 2000

Juli 2002 - Juli 2003

11. Juli 2003

Nov. 2003 - Jan. 2007
Katharina-Anna Klapa

04.03 .1978

Kowary

Richard und Maria Klapa, geb. Jabłoñska

deutsch

ledig

Grundschule in Soest

Grundschule in Kassel-Waldau

Grundschule in Baunatal

Wilhelmsgymnasium in Kassel

Allgemeine Hochschulreife Göttingen

Diplom-Chemiker-Vorprüfung

Diplomarbeit am Institut für Organische und Biomolekulare Chemie der Universität Göttingen unter Anleitung von Prof. Dr. Dr. h.c. C. Schneider über das Thema "Zirkoniumkatalysierte Tishchenko-Reduktion von $\beta$-Hydroxyketonen" Diplom-Chemiker-Hauptprüfung

Dissertation am Institut für Organische und Biomolekulare

Chemie der Universität Göttingen unter Anleitung von Prof. Dr. Dr. h.c. L. F. Tietze über das Thema "Untersuchungen zur Synthese von Yohimban- und Camptotheca-Alkaloiden durch Domino-Knoevenagel-hetero-Diels-Alder-Reaktion” 
$\longrightarrow$

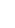

